\title{
Joint Development of
}

Seismic Capability Evaluation Technology for Degraded Structures and Components

Annual Report for Year 1 Task

\section{Identification and Assessment of Recent Aging-Related Degradation Occurrences in U.S. Nuclear Power Plants}

November 2008

Jinsuo Nie, Joseph Braverman, and Charles Hofmayer

Brookhaven National Laboratory

Upton, NY 11973, USA

Young-Sun Choun, Min Kyu Kim, and In-Kil Choi

Korea Atomic Energy Research Institute

Daejeon, 305-353, Korea 


\section{NOTICE/DISCLAIMER}

This manuscript has been authored by employees of Brookhaven Science Associates, LLC under Contract No. DE-AC02-98CH10886 with the U.S. Department of Energy. The United States Government retains a non-exclusive, paid-up, irrevocable, world-wide license to publish or reproduce the published form of this manuscript, or allow others to do so, for United States Government purposes.

Neither the United States Government nor any agency thereof, nor any of their employees, nor any of their contractors, subcontractors, or their employees, makes any warranty, express or implied, or assumes any legal liability or responsibility for the accuracy, completeness, or any third party's use or the results of such use of any information, apparatus, product, or process disclosed, or represents that its use would not infringe privately owned rights. Reference herein to any specific commercial product, process, or service by trade name, trademark, manufacturer, or otherwise, does not necessarily constitute or imply its endorsement, recommendation, or favoring by the United States Government or any agency thereof or its contractors or subcontractors. The views and opinions of authors expressed herein do not necessarily state or reflect those of the United States Government or any agency thereof. 


\begin{abstract}
In the past, safety assessments of nuclear power plants (NPPs), using methods such as seismic probabilistic risk assessments (PRAs), usually utilized best estimate or design values for material properties of structures, systems, and components (SSCs) without consideration of any aging effects. In order to develop a realistic evaluation of the seismic safety of a plant, the potential effects of age-related degradation on (SSCs) should be considered.

To address the issue of aging degradation for the safety of NPPs, the Korea Atomic Energy Research Institute (KAERI) has embarked on a five-year research project to develop a realistic seismic risk evaluation system which will include the consideration of aging of structures and components in NPPs. Three specific areas that are included in the KAERI research project, related to seismic PRA, are probabilistic seismic hazard analysis, seismic fragility analysis including the effects of aging, and a plant seismic risk analysis.

To support the development of seismic capability evaluation technology for degraded structures and components, KAERI entered into a collaboration agreement with Brookhaven National Laboratory (BNL) in 2007. The collaborative research effort is intended to continue over a five year period with the goal of developing seismic fragility analysis methods that consider the potential effects of age-related degradation of SSCs, and using these results as input to seismic PRAs.

This report describes the research effort performed by BNL for the Year 1 scope of work. This research focused on collecting and reviewing degradation occurrences in US NPPs and identifying important aging characteristics needed for the seismic capability evaluations that will be performed in the subsequent evaluations in the years that follow. The report presents results of the statistical and trending analysis of this data and compares the results to prior aging studies. In addition, this report provides a description of current regulatory requirements, regulatory guidance documents, generic communications, industry standards and guidance, and past research related to aging degradation of SSCs. Finally, this report provides the conclusions reached from this research effort, which includes a summary of the findings from the identification and evaluation effort of degradation occurrences, an assessment of the degradation trending results, and insights into the important aging characteristics that should be considered in the tasks to be performed in the Year 2 through 5 research efforts.
\end{abstract}





\section{TABLE OF CONTENTS}

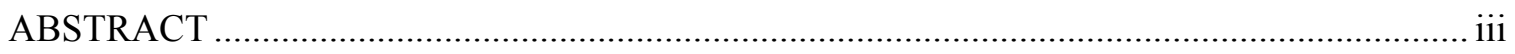

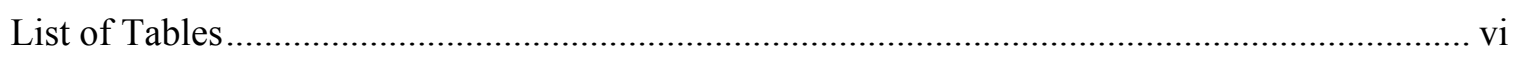

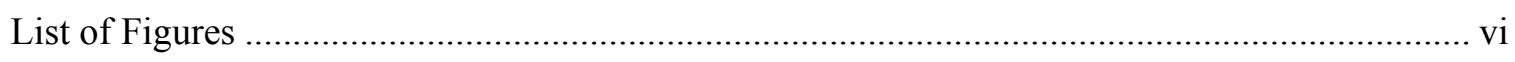

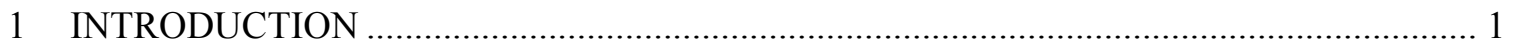

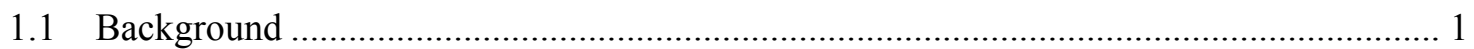

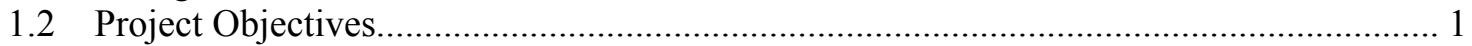

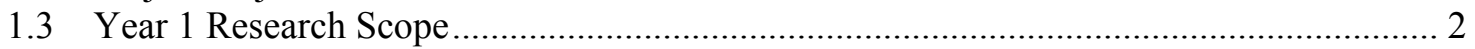

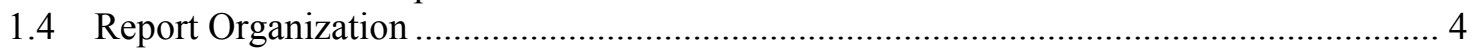

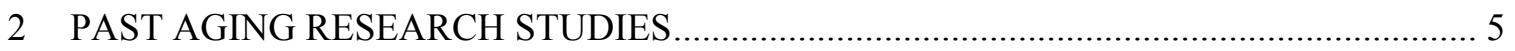

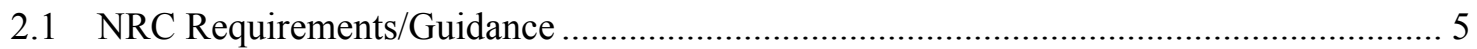

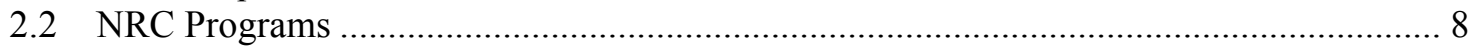

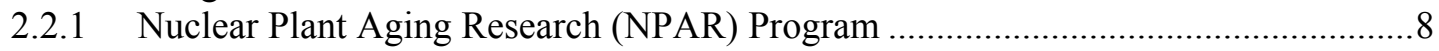

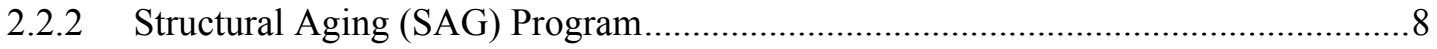

2.2.3 Nuclear Power Plant Generic Aging Lessons Learned (GALL) ............................... 9

2.2.4 Assessment of Inservice Conditions of Safety-Related Nuclear Plant

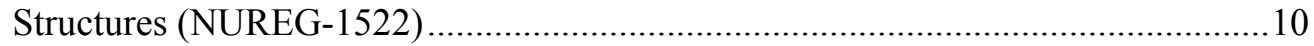

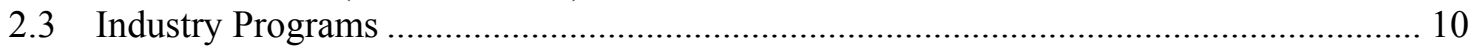

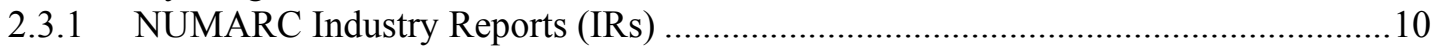

2.3.2 NEI - Industry Guideline for Monitoring the Effectiveness of Maintenance at Nuclear Power Plants .......................................................................................... 11

2.3.3 American Concrete Institute (ACI) Codes and Standards...................................... 11

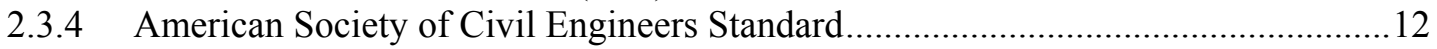

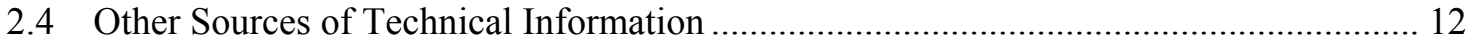

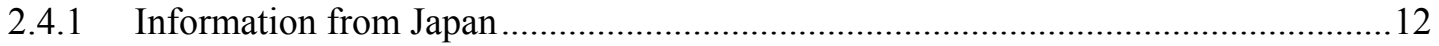

2.4.2 Organization for Economic Co-operation and Development (OECD) -

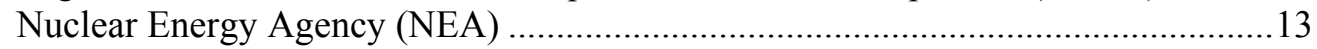

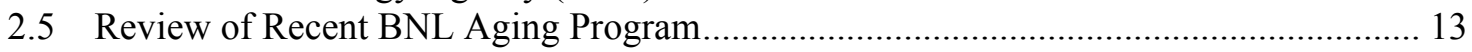

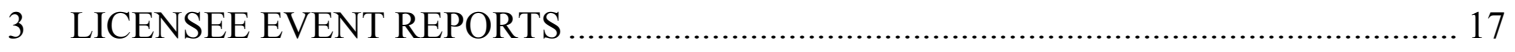

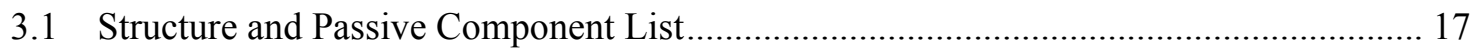

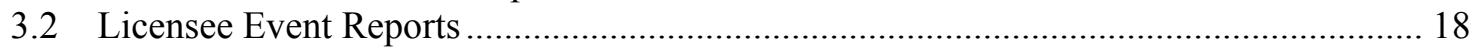

3.3 Keyword Search Approach through NRC ADAMS System......................................... 19

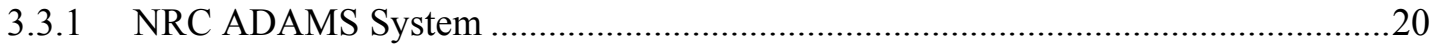

3.3.2 Development of the Keyword Search Approach...................................................20

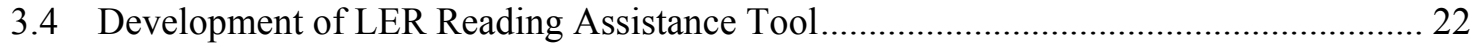

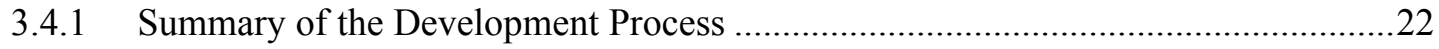

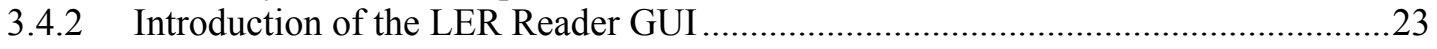

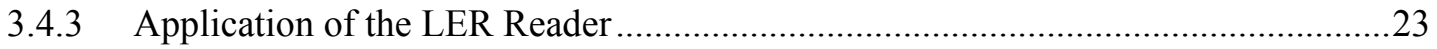

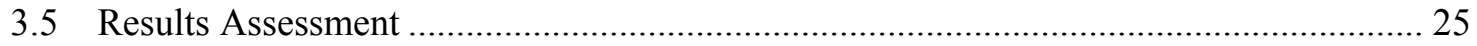

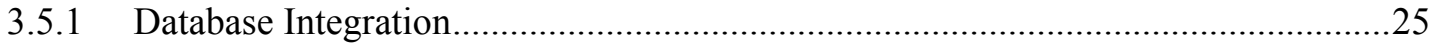

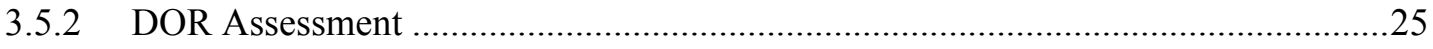

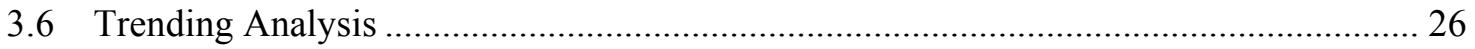

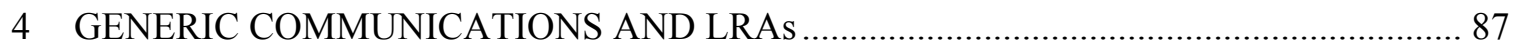

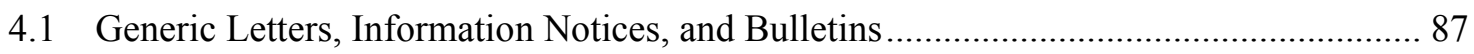

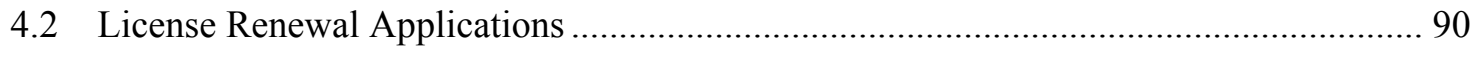

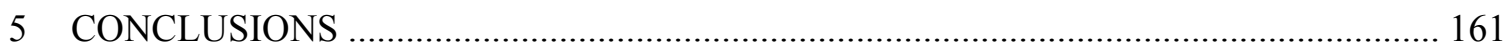




\section{LIST OF TABLES}

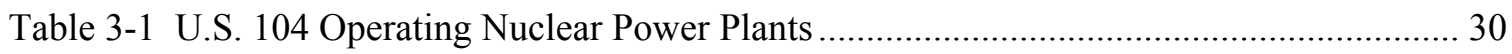

Table 3-2 Degradation Occurrence Records (Primary Information) ........................................... 34

Table 3-3 Degradation Occurrence Records (Secondary Information) ........................................ 48

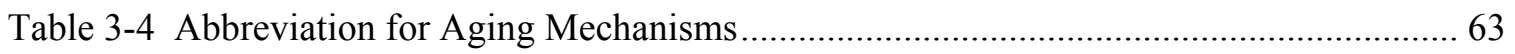

Table 3-5 Number of DORs and NPP Units by PAAE for LER 1999-2007 ............................... 64

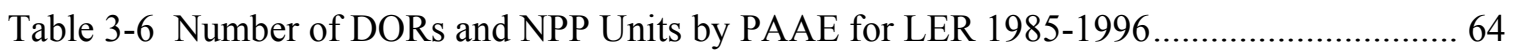

Table 4-1 NRC Generic Communications for Information Related to Aging Degradation ........ 93

Table 4-2 Summary of Degradation Information by Generic Communications ........................ 142

Table 4-3 Summary of Degradation by Components ............................................................ 143

Table 4-4 License Renewal Applications for Information Related to Aging Degradation -

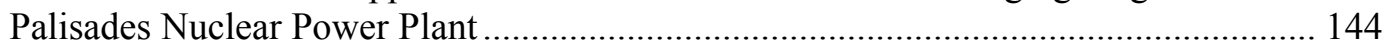

Table 4-5 License Renewal Applications for Information Related to Aging Degradation Point Beach Nuclear Power Plant Units $1 \&$ 2 ........................................................... 151

\section{LIST OF FIGURES}

Figure 3-1 A Sample LER in Text Format (Beginning Part) ................................................ 65

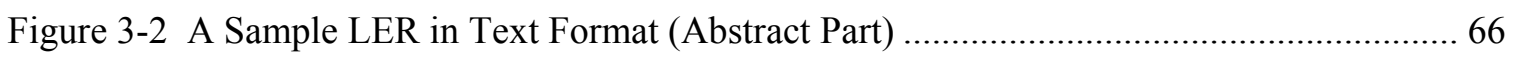

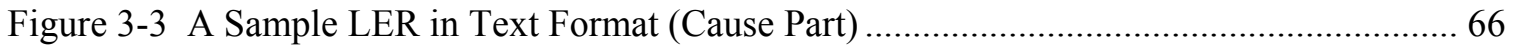

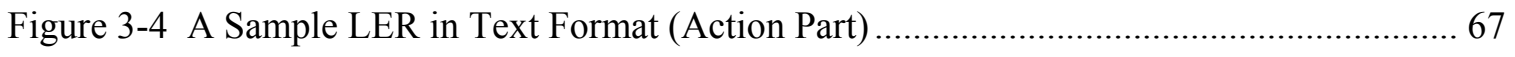

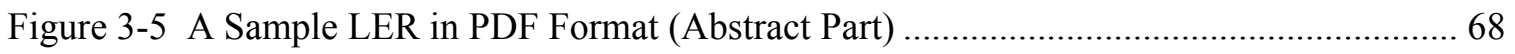

Figure 3-6 The Annotated LER Reader Graphical User Interface ........................................... 69

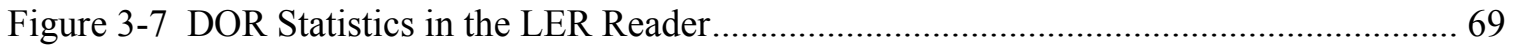

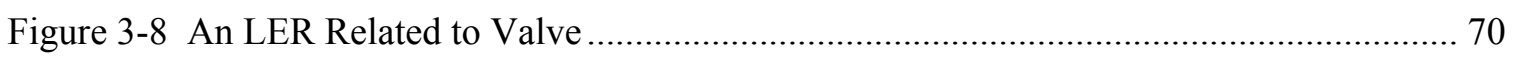

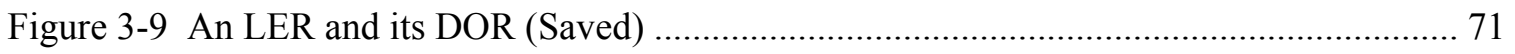

Figure 3-10 An LER Required Careful Review .................................................................. 72

Figure 3-11 An LER Possibly Related to a Design Problem (Required Careful Review) ........... 73

Figure 3-12 An LER Related to Human Error and Electric Parts ........................................... 74

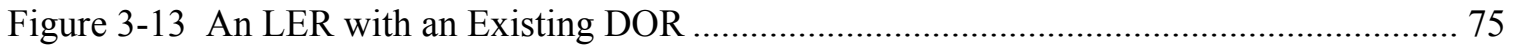

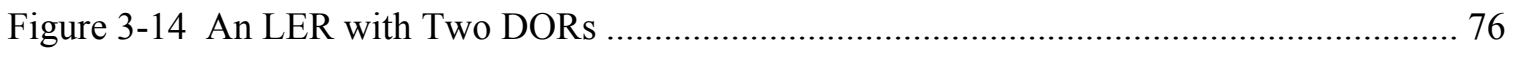

Figure 3-15 An LER Related to Human Error and Connectors................................................. 77

Figure 3-16 An LER Related to Concrete Degradation ........................................................ 78

Figure 3-17 Distribution of 104 Operating US NPPs in Four NRC Regions [NRC Website] ..... 79

Figure 3-18 Distribution of SPC Degradation Occurrences over Component Category .............. 79

Figure 3-19 Distribution Comparison of SPC Degradation Occurrences over Component......... 80

Figure 3-20 Comparison of Normalized Distribution of SPC Degradation Occurrences over

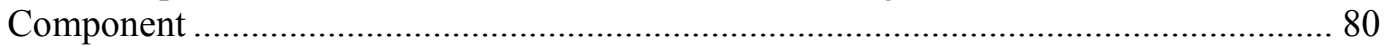


Figure 3-21 Distribution Comparison of SPC Degradation Occurrences over Time

Figure 3-22 Number of Degradation Occurrences and NPP Units with Plant Age at Event ....... 82

Figure 3-23 Number of Degradation Occurrences and NPP Units with PAAE for Combined

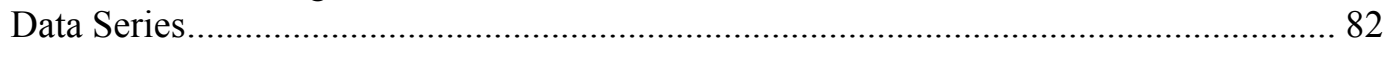

Figure 3-24 Average Degradation Occurrences with Plant Age at Event ................................. 83

Figure 3-25 Relation of Average DORs per Plant versus PAAE with Combined Data Series .... 83

Figure 3-26 Distribution Comparison of SPC Degradation Occurrences over Aging Effect

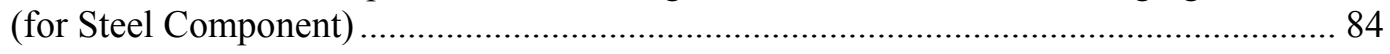

Figure 3-27 Distribution Comparison of SPC Degradation Occurrences over Aging

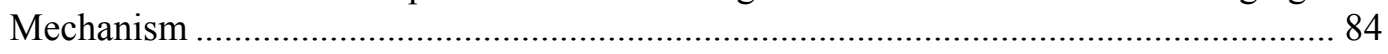

Figure 3-28 Distribution Comparison of SPC Degradation Occurrences over Cracking Type.... 85

Figure 3-29 Distribution Comparison of SPC Degradation Occurrences over System................ 85 



\section{INTRODUCTION}

\subsection{Background}

The Korea Atomic Energy Research Institute (KAERI) is in the process of performing research to improve the evaluation methods to assess the seismic safety of new and existing operating power plants. These evaluation methods can be used to perform Periodic Safety Reviews (PSRs) and license renewal application (LRA) reviews. In addition, they can also be used to upgrade the seismic safety of a nuclear power plant (NPP). In the past, such evaluation methods included a seismic probabilistic risk assessment (PRA) which usually utilized design values without consideration of any aging effects. In order to develop a realistic evaluation of the seismic safety of a plant, the potential effects of age-related degradation on structures, systems, and components (SSCs) within a NPP should be considered.

To address the issue of aging degradation on the safety of NPPs, KAERI has embarked on a fiveyear research project to develop a realistic seismic risk evaluation system which will include the consideration of aging of structures and components in NPPs. Three specific areas that are included in the KAERI research project, related to seismic PRA, are probabilistic seismic hazard analysis, seismic fragility analysis by considering age-related degradation, and a plant seismic risk analysis. The major objectives of the KAERI project are:

- Reduction of the uncertainty in a PSHA (Probabilistic Seismic Hazard Analysis)

- Development of site-specific evaluation response spectra for a seismic PRA

- Development of seismic fragility analysis methodology by considering a realistic capacity and response of structures and components that includes the effects of aging

- Development of time-dependent and nonlinear analysis technology for nuclear structures and components

- Development of seismic risk quantification models and tools for Korean NPPs

- Evaluation of the aging effect on the seismic risk of a NPP

To meet the above objectives, KAERI entered into a collaboration agreement with Brookhaven National Laboratory (BNL) in 2007. The collaborative research effort is intended to continue over a five year period with the goal of developing seismic capability evaluation technology for degraded structures and components. The final results of this project will be utilized to update and improve the seismic PRA technology in Korea. The advanced seismic PRA tools developed by KAERI with input from the research performed by BNL, will be used for evaluations of the seismic safety of operating NPPs in Korea during the process of their PSR, for license renewal evaluations of old NPPs, and for future plants.

\subsection{Project Objectives}

The objective of the research effort being performed by BNL, as part of the collaborative research agreement, is to provide technical assistance to KAERI in developing seismic capability evaluation technology for degraded structures and components, which can be used to assess the safety of NPPs in Korea. This will be achieved by conducting research in the area of how agerelated degradation effects can be considered in performing seismic PRAs of NPPs. The research effort by BNL will be conducted over a five-year period from 2007 through 2012. The ultimate goal of the development of seismic capability evaluation technology for degraded structures and components is divided into five sets of individual objectives associated with the each of the five 
years. The objectives of the individual tasks to be performed in each of the five years is described below.

\section{$\underline{\text { Year } 1 \text { Objectives: }}$}

The Year 1 objective is for BNL to collect and review degradation occurrences in US NPPs and identify important aging characteristics needed for the seismic capability evaluations that will be performed in the subsequent evaluations in the years that follow. It is anticipated that aging characteristics such as the component type, aging effects, aging mechanisms, identification method, evaluation method, plant name, and date of occurrence would be considered for review. The information presented in this report provides a description of the research effort performed by BNL to meet the Year 1 objectives.

\section{Year 2 Objectives:}

The Year 2 objective is for BNL to identify modeling methodologies to represent the long-term behavior of materials used in NPPs. BNL will perform a literature search for time-dependent models that can approximate the degradation effects of the key materials used for the structures and passive components. It is envisioned that the degradation models identified would potentially cover the most common time-dependent changes in material properties (e.g., strength, ductility, modulus), loss of material (e.g., corrosion, erosion), and cracking.

\section{Year 3 Objectives:}

The Year 3 objective is for BNL to select one structure or component, with prior approval from KAERI, and develop the seismic fragility capacity. The seismic fragility for this structure or component will be developed for the undegraded condition and various levels of degradation for the most common aging effect identified in the earlier Year 1 Task described above. The intent of this task is to provide a pilot study that demonstrates how the seismic fragility calculation methodology can be performed.

\section{$\underline{\text { Year } 4 \text { Objectives: }}$}

The Year 4 objective is for BNL to provide technical assistance to KAERI staff who will perform seismic fragility calculations for the other remaining important structures and components. The seismic fragility calculation for the other important structures and components are not necessarily expected to be the same as that performed in the Year 3 Task, due to the differences in the structure/components, materials, aging effects, and/or failure modes.

\section{$\underline{\text { Year } 5 \text { Objectives: }}$}

The Year 5 objective is for BNL to provide technical assistance to KAERI to develop guidance for establishing degradation acceptance criteria for structures and components. This may follow a similar approach that was utilized in the NRC aging research project that was performed by BNL recently and is reported in NUREG/CR-6715 and NUREG/CR-6876. The focus of BNL assistance will be on providing expertise in structural analysis and fragility analysis.

\subsection{Year 1 Research Scope}

The scope of the Year 1 research effort was to identify, collect and evaluate age-related degradation occurrences in United States (US) NPPs. This required developing a list of structures 
and components suitable for KAERI's seismic capability evaluations. Then, degradation occurrences for these structures and components were identified and reviewed. This information was evaluated in order to identify the important aging characteristics, and finally a trending analysis was performed to make a comparison to the results of past aging studies and to plan for the future tasks under the five-year research project.

A list of applicable structures and components was developed, with KAERI's approval, in order to define the scope of what types of structures/components should be reviewed for degradation occurrences. Similar to the study reported in NUREG/CR-6679, that did not include active components, it was recommended that this list cover only structures and passive components (SPCs), but not active components. Active components such as pumps, valves, and electrical equipment are components which must move or change their state in order to perform their intended functions, and can typically be monitored for aging effects by monitoring their performance. By monitoring pressure, flow, electrical signal, etc., the potential aging degradation of active components can be identified, while for passive components, the extent of aging is usually difficult to identify and may even go undetected. In addition, active components are usually subjected to periodic inspection, testing, and maintenance where aging effects would be detected and corrected over time.

KAERI provided a list of structures and components that are considered to be the most risk significant by KAERI engineers from the work that has already been performed by KAERI. Reviewing this list along with the list of 18 SPCs described in NUREG/CR-6679, 10 categories of SPCs were identified for the scope of the degradation review to be included in this study for KAERI as follows:
1. Anchorage
2. Concrete
3. Containment
4. Exchanger
5. Filter
6. Piping system
7. Reactor pressure vessel (RPV)
8. Structural steel
9. Tank
10. Vessel

These SPC categories will be explained in more detail in Section 3 of this report.

The identification of degradation occurrences for these SPCs was performed using publicly available documents related to the nuclear power industry, primarily in the US. The documents reviewed for the current research program, reported herein, include Licensing Event Reports (LERs), US Nuclear Regulatory Commission (NRC) generic correspondences, documents related to license renewal activities, industry reports, NUREG reports, BNL and other national lab reports. LERs are reports that must be submitted by NPP licensees to the NRC if certain potential problems occur such as: any event or conditions that occurred in the plant which resulted in a condition of the plant being seriously degraded or the plant being in an unanalyzed condition that significantly degraded plant safety. More details about LERs are reported in Section 3 of this report.

The prior research reported in NUREG/CR-6679 collected and evaluated degradation occurrences for SPCs in US NPPs over the period 1985 to 1997. Therefore, the current BNL research for 
KAERI was intended to focus on the more current period from 1997 to the present. That way, there would not be any overlap and an assessment can be made for the current period and the results may be compared to the conclusions reached from the prior period.

\subsection{Report Organization}

This report consists of five sections. Section 2 presents a summary of the past aging research studies which include programs and studies conducted by BNL, NRC, and other research institutes and the nuclear industry. Although, describing this information was not intended to be part of this research task, it is useful to provide this description in the report to present a framework of what aging technology information is available from past studies and the regulatory requirements and guidance documents related to aging. Section 3 of the report describes the review of the recent LERs and the corresponding statistical and trending analysis. Section 4 summarizes the review of the recent generic communications and LRAs. Section 5 provides the conclusions reached from this research effort, which includes a summary of the findings from the identification and evaluation effort of degradation occurrences, and an assessment of the degradation trending analysis. 


\section{PAST AGING RESEARCH STUDIES}

There is substantial existing aging technology information from prior aging research studies and current industry programs. This includes both NRC and industry sponsored research programs. This section first describes NRC requirements/guidance related to aging degradation and then summarizes past NRC research programs, industry programs, and other technical information. Much of the information presented below is based on aging technology information reported in the prior BNL research program NUREG/CR-6679. Relevant information in this section, if not specially noted, refers to nuclear power plants in the U.S.

\subsection{NRC Requirements/Guidance}

This section summaries current NRC requirements and available guidance related to degradation of structures and passive components (SPCs), including requirements and guidance for containments, water-control structures, and masonry walls, and rules for maintenance and license renewal.

\section{Containments}

Periodic leak rate testing of containments is required in accordance with 10 CFR Part 50, Appendix J. The leak rate testing of a containment has three types of tests: A, B, and C. Type A tests are performed to measure the primary reactor containment overall integrated leakage rate, Type $\mathrm{B}$ tests are performed to detect local leaks for penetrations, and Type $\mathrm{C}$ tests are conducted to measure containment isolation valve leakage rates. Prior to performing a Type A test, a general visual inspection of the accessible interior and exterior surfaces of the containment structures and components must be performed to identify any evidence of structural deterioration which may affect either the containment structural integrity or leak-tightness.

Regulatory Guides 1.35 and 1.35 .1 provides additional requirements for prestressed concrete containments for ungrouted tendons. Regulatory Guide 1.35 describes a basis acceptable to the NRC staff for developing an appropriate inservice inspection and surveillance program for ungrouted tendons in prestressed concrete containments. Regulatory Guide 1.35 provides guidance for performing visual inspections, prestress monitoring tests (lift-off tests), tendon material tests and inspections, inspection of filler grease, evaluation of inspection results, and reporting requirements. Regulatory Guide 1.35 .1 provides a basis acceptable to the staff for developing appropriate presstressing tolerance bands for tendons so that these limits can be compared against the lift-off forces measured in the sample inspection program of Regulatory Guide 1.35 .

10 CFR 50.55a has been revised to provide more precise requirements, assure that the critical areas of containments are routinely inspected, and take corrective action for defects that could compromise a containment's structural integrity. The final rulemaking, which was effective on September 9, 1996, endorsed the 1992 Edition with 1992 Addenda of Section XI, Subsection IWE (Class MC Containments) and Subsection IWL (Class CC Containments) of the ASME Code. Since the final rulemaking, subsequent revisions of 10 CFR 50.55a have been issued and now the current version also endorses as acceptable the 1995 Edition with the 1996 Addenda, the 1998 Edition through the 2000 Addenda, and the 2001 Edition through the latest edition and addenda. All of these endorsements are subject to certain limitations and conditions. Licensees must incorporate Subsection IWE and Subsection IWL into inservice inspection programs for containments. The rulemaking includes exemptions from and additional requirements to those in Subsections IWE and IWL. 


\section{Water-Control Structures}

Water-control structures include structures such as intake structures, canals, dams, earthen embankments and slopes associated with emergency cooling water systems or flood protection. For these structures, Regulatory Guide 1.127 describes a basis acceptable to the staff for developing an appropriate inservice inspection and surveillance program. Guidance is provided for the compilation of engineering data, onsite inspection program, technical evaluation, frequency of inspections, and preparation of reports.

\section{Masonry Walls}

A major re-evaluation effort of masonry walls in the nuclear industry was initiated by the NRC IE Bulletin 80-11 "Masonry Wall Design." This effort initiated by the bulletin was intended to demonstrate the structural adequacy of reinforced and unreinforced masonry walls. Of the seventy plants originally in the scope of 80-11, two were shut down; three were reviewed under the Systematic Evaluation Program (SEP); one plant had no safety-related masonry walls; four were qualified by analytical methods verified by full-scale testing; and the remaining sixty plants were qualified in accordance with the Structural Engineering Branch (SEB) Interim Criteria.

NRC issued Information Notice No. 87-67, "Lessons Learned from Regional Inspections of Licensee Actions in Response to IE Bulletin 80-11," in December 1987. This notice described a number of deficiencies uncovered during site audits, which were grouped as:

- Unanalyzed Conditions - existing cracks in unreinforced masonry

- Improper Assumptions - mortar properties, boundary conditions, presence of reinforcement

- Improper Classification - specification of safety-related versus non-safety walls

- Lack of Procedural Controls - walk-down surveys, record keeping, modification activities

The information notice also indicated that "NRC inspectors observed that mechanisms did not exist at certain facilities to ensure that the physical conditions of masonry walls remained as previously analyzed."

Then an internal NRC report entitled, "Status of Multi-Plant Action (MPA) B-59, Masonry Wall Design" was issued in August 1988. This report recommended that the MPA be considered closed and also summarized the current status of each plant included in the action. The report also stated that the Office of Inspection and Enforcement had responsibility of inspection related activities.

\section{CFR 50.65 - Maintenance Rule}

Another important regulation, 10 CFR 50.65 entitled, "Requirements for Monitoring the Effectiveness of Maintenance at Nuclear Power Plants," was published by the NRC on July 10, 1991. This regulation, which was referred to as the Maintenance Rule, became effective on July 10, 1996. The goal of the Maintenance Rule is to monitor the effectiveness of maintenance activities for safety significant plant equipment in order to minimize the likelihood of failures and abnormal events caused by the lack of effective maintenance. The final rule requires that licensees monitor the performance or condition of structures, systems, and components (SSCs) against licensee-established goals in a manner sufficient to provide reasonable assurance that the SSCs will be capable of performing their intended functions. Such monitoring needs to be established commensurate with safety and, where practical, take into account industry operating experience. 
Other documents that provide additional technical information and guidance, related to the Maintenance Rule, include: Regulatory Guide 1.160, Rev. 2, "Monitoring the Effectiveness of Maintenance at Nuclear Power Plants;" NUMARC 93-01, Rev. 2, "Nuclear Energy Institute Industry Guideline for Monitoring the Effectiveness of Maintenance at Nuclear Power Plants;" NRC Inspection Manual - Inspection Procedure 62706, "Maintenance Rule;" NRC Inspection Manual - Inspection Procedure 62002, "Inspection of Structures, Passive Components, and Civil Engineering Features at Nuclear Power Plants;" and NRC Inspection Manual - Inspection Procedure 62003, "Inspection of Steel and Concrete Containment Structures at Nuclear Power Plants."

\section{CFR Part 54 - License Renewal Rule}

Nuclear power plants in the U.S. were initially licensed to operate for 40 years. To extend the life of these plants, requirements for obtaining the renewal of an operating license, for an additional 20 years, are presented in 10 CFR Part 54 - License Renewal Rule. Under 10 CFR Part 54, applicants are required to identify all SSCs that are within the scope of the rule. A screening review is then required to identify those SSCs that are "passive and long-lived" structures and components. For the passive, long-lived structures and components, the applicant must demonstrate that the effects of aging will be managed so that the intended function(s) will be maintained consistent with the current licensing basis through the period of extended operation.

In addition, applicants are also required to identify and update all time-limited aging analyses (TLAAs) which are part of the current licensing basis. An example would be a design basis fatigue analysis of a piping system which assumed a specified number of loading events based on a 40-year period of operation. For prestressed concrete containments, a TLAA is required to demonstrate that the prestressing tendons, which lose the tendon prestress loads over time, are still adequate for the additional 20 years of operation beyond the initial 40 years assumed in design.

In September 2005, the NRC issued Regulatory Guide 1.188, Rev. 1, "Standard Format and Content for Applications to Renew Nuclear Power Plant Operating Licenses." This regulatory guide indicates that the application should include (1) general information, (2) an integrated plant assessment (IPA), (3) an evaluation of time-limited aging analyses (TLAAs), (4) a supplement to the plant's final safety analysis report (FSAR), (5) any necessary changes to the plant's technical specifications (along with related justifications), and (6) a supplement to the plant's environmental report. The FSAR supplement should provide a summary description of the programs and activities that the applicant will use to manage the effects of aging for the period of extended operation, which is determined by the IPA and the evaluation of TLAAs. This regulatory guide also endorses the industry guidance document NEI 95-10, Revision 6 for use in implementing the license renewal rule.

Another NRC document that provides guidance for license renewal of NPPs is NUREG-1800, Revision 1, "Standard Review Plan for the Review of License Renewal Applications for Nuclear Power Plants" (SRP-LR), published in September 2005. This SRP-LR was prepared to provide guidance for staff reviewers in performing safety reviews of applications to renew licenses of nuclear power plants in accordance with 10 CFR Part 54. A companion document also published by the NRC in September 2005 is NUREG-1801, Revision 1, entitled, Generic Aging Lessons

Learned (GALL) Report. This two volume document provides very specific guidance for use by applicants and the NRC staff reviewers to ensure effective, efficient and consistent satisfaction of the LR Rule requirements. The revised SRP-LR references the GALL Report for descriptions of generic aging management programs which the staff has evaluated and found applicable to license renewal. 


\subsection{NRC Programs}

Some of the major NRC research programs related to aging degradation of structures and passive components (SPCs) in nuclear power plants are summarized below. A more complete listing of the NRC programs in this area is presented in NUREG/CR-6679.

\subsubsection{Nuclear Plant Aging Research (NPAR) Program}

In 1985 the NRC sponsored a research program to identify and resolve technical safety issues related to aging of SSCs in operating nuclear power plants. The principal goals of the program were to understand the effects of age-related degradation in NPPs and how to manage and mitigate them effectively. NUREG-1144, Rev. 2 describes the objectives of the program, the current status of research, and summarizes the utilization of the research results in the regulatory process. As a result of the NPAR program approximately 100 NUREG/CR reports have been developed as of June 1991, plus numerous published papers and proceedings.

A listing of past research activities under the NPAR program through September 1993 is presented in NUREG-1377, Rev. 4. This NUREG contains summaries of NRC sponsored reports that were generated in the NPAR Program. Each summary describes the objectives of the research, the contractor, and authors, and outlines significant research results. Although most of the items included in this NUREG cover hardware oriented plant components and systems, there are some summaries given for structural and passive components.

\subsubsection{Structural Aging (SAG) Program}

In 1988, the NRC sponsored a major research program on structural aging referred to as SAG. The objective of the SAG Program was to develop the technical bases for addressing aging of safety-related concrete structures and providing guidance for use in evaluating continued service of these concrete structures. Over 90 technical reports and papers have been published describing the results of the program.

The SAG Program consisted of a management task and three technical task areas. The objective of the management task was to effectively manage the technical tasks related to the safety issues of aging NPP concrete structures. The first technical task was to develop a materials property database. This consisted of a reference source containing data and information on the time variation of material properties under exposure to applicable environmental stressors (mechanisms) and aging factors. The materials database covered various concrete types, steel reinforcements, prestressing tendons, structural steels, and rubber materials. The information contained in the database can be used to predict deterioration of structural components in NPPs and in developing limits on detrimental environmental exposures.

The second technical task described a methodology that can be used to (1) make quantitative assessments of environmental stressors or aging factors that could affect safety-related concrete structures at NPPs and (2) provide recommended in-service inspection (ISI) or sampling procedures for use in evaluating the structural condition and for trending the performance of these components. Also included in this task are the identification and evaluation of techniques for mitigation of stressors or aging factors that may affect critical concrete components, and an assessment of techniques for repair, replacement, or retrofitting of deteriorated concrete components. 
The third technical task developed a quantitative methodology for continued service determinations. This included development of predictive models to assess the current and future reliability and performance of concrete structures.

A summary of the entire SAG Program is provided in NUREG/CR-6424. This report describes the SAG Program including a description of safety-related concrete structures and longevity considerations; inservice inspection, condition assessment, and remedial measure considerations; evaluation of NPP reinforced concrete structures; reliability-based methodology for condition assessments; and summary, conclusions, and recommendations. The NUREG includes an excellent description of the aging mechanisms and aging effects for concrete and associated steel components of reinforced concrete structures. Appendix B to the NUREG provides a listing of the numerous reports and papers that were developed under the SAG Program.

Some of the conclusions as reported in NUREG/CR-6424 are:

- The performance of the reinforced concrete structures in NPPs has been good. However, as these structures age, incidences of degradation due to environmental stressor effects are likely to increase to potentially threaten their durability. Items of note would be corrosion of steel reinforcement due to carbonation of the concrete or presence of chloride ions, excessive loss of prestressing force, leaching of concrete, and leakage of post-tensioning system corrosion inhibitor through cracks in the concrete.

- Techniques for detecting the effects of environmental stressors are sufficiently developed to provide qualitative data.

- Methods for conducting condition assessments of reinforced concrete structures are fairly well established. Few standards or criteria are available for interpreting the results obtained from condition assessments. Current inspection requirements for NPP reinforced concrete structures are fairly limited with the exception of concrete containments.

- Techniques for repair of concrete structures are well established and when properly selected and applied are effective. At the time, no codes or standards are available for repair of reinforced concrete structures, although some are being developed. Criteria that may be used to determine when a repair action should be implemented are not available.

- A reliability-based methodology has been developed that can be used to facilitate quantitative assessments of current and future structural reliability and performance of reinforced concrete structures in NPPs.

\subsubsection{Nuclear Power Plant Generic Aging Lessons Learned (GALL)}

Another important research effort sponsored by the NRC is presented in NUREG/CR-6490 entitled, "Nuclear Power Plant Generic Aging Lessons Learned (GALL)." This report describes the research effort to perform a systematic review of plant aging information in order to assess materials and component aging issues related to continued operation and license renewal of operating plants. A literature review was performed for mechanical, structural, thermal-hydraulic components and systems, and electrical components and systems.

The results of these reviews were tabulated and included in a two-volume report. The NUREG concluded, "all ongoing significant component aging issues are currently being addressed by the regulatory process. However, the aging of what are termed passive components have been highlighted for continued scrutiny." The NUREG lists the aging issues significant to passive 
components. Most of the structural components evaluated pertain to the RPV (instrumentation and CRD housing nozzles, closure studs, jet pump and holddown beams, reactor internals, core shroud, etc.); piping and feedwater nozzles and interfacing tanks and components; concrete shield walls; and other concrete elements.

The NUREG also concluded, "passive components are not as extensively or thoroughly covered by current plant maintenance procedures. Furthermore, surveillance and monitoring methods and instrumentation and procedures have not been as extensively developed or employed for passive components subjected to the highlighted aging mechanisms, nor are some of the passive component aging mechanisms as well understood." In addition, the NUREG points out that passive components are often the most costly and most difficult to replace. Therefore, the knowledge base for predicting applicable aging effects behavior and significance is very important for passive components.

\subsubsection{Assessment of Inservice Conditions of Safety-Related Nuclear Plant Structures (NUREG-1522)}

NUREG-1522, entitled "Assessment of Inservice Conditions of Safety-Related Nuclear Plant Structures" was published by the NRC in June 1995. This report describes the condition of structures and civil engineering features at operating nuclear power plants and provided information that would help identify, monitor, and correct degraded conditions of these structures. The NUREG contains descriptions of age-related degradation, which were obtained from many different sources. The most significant information came from site visits, conducted by the NRC staff and its contractor BNL, at six older nuclear power plants (licensed before 1977).

Some of the observations noted in the report identify certain types of structures (e.g. water intake structures, masonry walls, anchorages, tanks, buried piping, and inaccessible areas) as requiring special considerations. The report also concludes that based on the observations and information collected, structures and civil engineering features should be periodically inspected and a systematic maintenance program be implemented to ensure the expected useful life of the structures.

\subsection{Industry Programs}

\subsubsection{NUMARC Industry Reports (IRs)}

Nuclear Management and Resources Council (NUMARC), under the sponsorship by the DOE and EPRI directed the preparation of ten industry reports (IRs). The IRs covered items such as PWR and BWR vessels, internals, primary coolant boundary, containments, and Class I structures. The purpose of the IRs is to address age-related degradation of these components on a generic basis. The IRs would provide the technical basis, which could be referenced by licensees in support of their license renewal application.

Each IR identifies the components that comprise the subject item (e.g. BWR containment) and evaluates each component in terms of possible age-related degradation mechanisms. Thus, certain aging mechanisms were eliminated and only those age-related degradation mechanisms that could affect the component were identified and described. In addition, the IRs evaluated the capability of programs to manage aging mechanisms that are applicable, and where generic effective programs cannot be shown to be capable of managing the effects of age-related degradation, aging management options for plant-specific programs are described. 


\subsubsection{NEI - Industry Guideline for Monitoring the Effectiveness of Maintenance at Nuclear Power Plants}

The Nuclear Energy Institute (NEI) has developed an industry guidance document (NUMARC 93-01, Rev. 2) entitled, "Industry Guideline for Monitoring the Effectiveness of Maintenance at Nuclear Power Plants." This guideline was developed to assist the industry in implementing the Maintenance Rule (10 CFR 50.65). The guideline describes the process for the identification of the SSCs within the scope of the Maintenance Rule and the process of establishing plant-specific risk significant criteria and performance criteria.

NUMARC 93-01 provides guidelines that include methodologies to select plant structures, systems, and components; establishing risk and performance criteria/goal setting and monitoring; identification of SSCs subject to effective preventive maintenance programs; evaluation of systems to be removed from service; and periodic maintenance effectiveness assessments.

The NUMARC document specifically addresses monitoring of structures under the Maintenance Rule (MR). The applicability of the MR to structures was a subject of considerable confusion within the industry during initial implementation of the MR. It is clearly stated in Section 10.2.3 of NUMARC 93-01, that structures which perform intended functions, in accordance with the criteria provided in NUMARC 93-01, are within the scope of the MR and require a monitoring program which ensures that degradation is detected before there is loss of any intended function.

NRC Regulatory Guide 1.160, Rev. 2 endorses NUMARC 93-01, Rev. 2 as an acceptable method to satisfy the general requirements of the Maintenance Rule. Regulatory Guide 1.160, Rev. 2 also addresses monitoring of structures under the Maintenance Rule and provides specific guidance for satisfying the requirements of the Maintenance Rule, as it pertains to structures.

\subsubsection{American Concrete Institute (ACI) Codes and Standards}

The American Concrete Institute (ACI) has developed a number of codes and standards that relate to degradation of reinforced concrete structures. ACI 201.1R-68, "Guide for Making a Condition Survey of Concrete in Service" provides a system for reporting on the condition of concrete in service. This guide includes a checklist for making a survey of the condition of concrete, provides a definition of the terms associated with the durability of concrete, and presents actual photographs to demonstrate the different types of aging effects.

ACI 201.2R-77, "Guide to Durable Concrete" discusses the more important causes of concrete degradation and gives recommendations on how to prevent such damage. Topics covered include freezing and thawing, aggressive chemical exposure, abrasion, corrosion of steel and other materials embedded in concrete, chemical reactions of aggregates, repair of concrete, and the use of coatings to enhance concrete durability.

ACI 207.3R-79, "Practices for Evaluation of Concrete in Existing Massive Structures for Service Conditions" describes methods for evaluating the physical properties of concrete in existing concrete structures. The report covers the review of preconstruction data, construction, operation and maintenance records; review of in-service inspections; condition surveys; nondestructive testing; and destructive testing.

ACI 224.1R-93, "Causes, Evaluation, and Repair of Cracks in Concrete Structures" summarizes the causes of cracks in concrete and the means for their control. The report also describes 
evaluation procedures and methods for crack repair such as epoxy injection, routing (enlarging the crack) and sealing, stitching (U-shaped metal units), use of additional reinforcement, and grouting.

ACI 349.3R-96, "Evaluation of Existing Nuclear Safety-Related Concrete Structures" presents recommendations for developing an effective evaluation procedure for nuclear safety-related concrete structures. The report describes the selection process of critical structures, the various degradation mechanisms, inspection techniques, evaluation criteria, evaluation frequency, qualifications of evaluation team, and repairs. Under the evaluation criteria recommendations, ACI 349.3R-96 presents a three tiered evaluation criteria: acceptance without further evaluation, acceptance after review, and conditions requiring further evaluation. It is in this area that the technical basis for some of the acceptance criteria need to be developed, expanded, and documented.

Other ACI standards such as ACI 224R-90, "Control of Cracking in Concrete Structures" and 222R-89, "Corrosion of Metals in Concrete" are listed in the Reference section of ACI 349.3R-96. ACI 349.3R-96 also lists related standards from ASCE, ASME, and ASTM.

\subsubsection{American Society of Civil Engineers Standard}

American Society of Civil Engineers (ASCE) Standard ASCE 11-90, "Guideline for Structural Condition Assessment of Existing Buildings" provides guidelines and a methodology for the structural assessment of existing buildings. Assessment techniques are provided for conventional buildings (non-nuclear) constructed from materials consisting of concrete, metals, masonry, and wood. The standard describes assessment procedures, condition assessment of materials, and evaluation procedures. Tables are presented in the guideline, which provide for each test method, a description of the application, principle of operation, user expertise, advantages, limitations, and references. Also included in the guideline are tables, which identify the various test methods which are most appropriate to evaluate chemical and physical properties of the material.

\subsection{Other Sources of Technical Information}

\subsubsection{Information from Japan}

Substantial technical information, regarding age-related degradation of structures and passive components is also available from international sources. A review of Japanese literature for degraded concrete structures was conducted by BNL under a separate research program for the NRC. A report by Park (September 1998) entitled "Effects of Aging Degradation on Seismic Performance of Reinforced Concrete Structures: Summary of Japanese Literature in Related Areas" summarizes the results of the review.

The 1998 report by Park provides a summary of a literature survey of available Japanese publications. Key observations are described in detail regarding age-related degradation mechanisms and seismic performance of degraded reinforced concrete structures. The report covers experimental studies on reinforced concrete members such as shear walls and beams in degraded conditions. Some of the observations and preliminary conclusions noted are:

- Vertical cracks in beams (normal to member axis) reduce the bending stiffness. However, vertical cracks do not significantly reduce the bending strength. Vertical cracks, in general, do not affect shear strength, unless they are located at the compression failure 
zone. Horizontal cracks (along component axis) affect the shear strength more than the bending strength.

- The orientation of cracks in concrete shear walls determines whether cracks affect the seismic capacity of components. Cracks would affect the shear capacity if they coincide with cracks caused by applied seismic loads or when they alter the failure mode.

- The size and number of cracks indirectly affect the seismic performance of all concrete structural members since the extent of corrosion is largely affected by crack size.

- There are indications that some initial levels of corrosion of steel reinforcement would increase the flexural strength of beams.

\subsubsection{Organization for Economic Co-operation and Development (OECD) - Nuclear Energy Agency (NEA)}

The Nuclear Energy Agency (NEA) is an intergovernmental body within the OECD located in Paris, France. The objective of the NEA is to assist its member countries in the development of nuclear energy as a safe, environmentally acceptable, and economical energy source through cooperation among its participating countries. Currently there are 28 countries including the United States that are members of the NEA. One of the committees within NEA, the Committee on the Safety of Nuclear Installations (CSNI) has a Working Group on Integrity of Components and Structures (IAGE). The main areas investigated by this working group include: the integrity of metal components; the integrity and ageing of concrete structures; and the seismic behavior of structures and components. A number of studies related to aging of structures and components were performed by the Working Group on IAGE and its predecessor the Principal Working Group (PWG-3) entitled "Integrity of Structures and Components."

An OECD - NEA Workshop on Finite Element (FE) Analysis of Degraded Concrete Structures was sponsored by the U.S. NRC and the OECD-NEA. This workshop was held at BNL on October 29-30, 1998. During the workshop over seventeen papers were presented related to the topic of the workshop. Many of the papers described technical approaches to utilize FE analysis methods for degraded concrete structures. A list of CSNI reports produced by or relevant to PWG-3 subgroups on the aging of concrete structures and the seismic behavior of structures is provided in NUREG/CR-6679.

\subsection{Review of Recent BNL Aging Program}

BNL has participated in a number of aging related research programs such as the NUREG-1522 study and technical assistance for NUREG-1801 (GALL). This section of the report will present a summary of an NRC sponsored BNL research study of aging degradation of NPP SPCs. A multi-year research program was performed to assess age-related degradation of SPCs for U.S. nuclear power plants. The objective of this program was to develop the technical basis for the validation and improvement of analytical methods and acceptance criteria which can be used to make risk-informed decisions and to address technical issues related to degradation of structures and passive components. This research program consisted of two phases. The Phase I effort included collection and evaluation of plant degradation occurrences, an assessment of the available technical information on age-related degradation, and a scoping study to identify which structures and passive components should be studied in the subsequent phases of the research program. Based on the results of the Phase I effort, selected SPCs were evaluated in Phase II to assess the effects of age-related degradation using existing and enhanced analytical methods. In 
addition, the Phase II effort included developing recommendations to the NRC staff for making risk-informed decisions related to degradation of structures and passive components.

\section{Phase I: Assessment of Age-Related Degradation of NPP SPC}

The description and results of the Phase I effort are reported in NUREG/CR-6679. Three activities were performed in this assessment effort. In the first activity, instances of age-related degradation were collected and evaluated. Licensee Event Reports, NRC generic communications, NUREG reports, and industry reports were reviewed to collect degradation data, which were stored in a database as summaries of important parameters. Then, trending analyses were performed to evaluate the data and develop important observations. The trending analyses provided conclusions such as what SPCs were most susceptible to age-related degradation, the most common aging mechanisms and aging effects, and the trend of the degradation occurrences over time. The second activity included the compilation of additional technical information related to aging such as NRC requirements/guidance, NRC programs, industry programs, degradation information from other countries, and other reports/papers on aging degradation. The third activity was a scoping study to identify those SPCs that warrant further detailed evaluation in Phase II. The scoping study was performed based on four key factors: degradation occurrences, seismic risk significance, adequacy of existing NRC and industry programs, and importance to current licensing basis/license renewal. The results of the scoping study determined that of the 18 original SPCs included in the research program, the five SPCs that should be included in the Phase II effort for more detail study are concrete structures (other than containment), buried piping, flat bottom atmospheric tanks, anchorages, and masonry walls.

It should be emphasized that a very extensive degradation reference database was created during the Phase I work which is presented in Appendix B to NUREG/CR-6679. This database covered the codes, industry standards, guidelines, NUREG reports, technical papers, presentations (at conferences), regulatory documents, and other reports that were collected and reviewed in Phase I. The regulatory documents included Code of Federal Regulations (CFRs); NRC generic correspondences such as Inspection and Enforcement Bulletins (IEs), Information Notices (INs), Generic Letters (GLs), etc.; NRC inspection reports; NRC regulatory guides; and NRC SECY papers. The reference database included over 160 documents in total, each of which consists of the type of document, the identification or ID (document no.), title of the document, date of publication, author/organization, a summary description, types of components covered, and potential aging issues identified in that document.

\section{Phase II: Detailed Evaluations of Identified Components for Assessment of the Effects of Age-Related Degradation on SPCs}

Of the five components that were identified during Phase I that warrant further detailed evaluation in Phase II, studies for two of them were completed. A detailed study was performed for reinforced concrete elements and buried piping and the results of these studies were presented in two NUREG reports. The objectives of the detailed studies in Phase II were to develop and improve analytical methods that can be used to assess the effects of age-related degradation on the structural performance of the SPCs, quantify the impact of age-related degradation of structures and passive components on overall plant risk, and develop degradation acceptance criteria that can be used to assist the staff in making risk-informed decisions for age-degraded SPCs. 


\section{$\underline{\text { Reinforced Concrete Components }}$}

NUREG/CR-6715 summarizes BNL's study that addressed the concerns related to aging degradation of reinforced concrete structures at NPPs. The aging effects due to reinforced concrete degradation mechanisms were studied and the corresponding analytical methods and degradation acceptance limits for concrete flexural and shear wall members were developed. Fragility modeling procedures for undegraded and degraded reinforced concrete structural components subjected to earthquake loadings were studied. These probability-based quantitative methods provided a basis for evaluation of reinforced concrete structures in nuclear plants for continued service and for development of guidelines for in-service inspection and maintenance. Probability-based degradation acceptance criteria (DAC) were also developed to assist the NRC staff in making risk-informed decisions regarding degradation of reinforced concrete components.

\section{Buried Piping}

NUREG/CR-6876 presents the results of the second detailed study performed by BNL which addressed the effects of degradation on buried piping. The purpose of this study was similar to the concrete study, which is to develop analytical methods that can be used to assess the effects of aging degradation of buried piping and to develop degradation acceptance criteria (DAC) that can be used to assess the condition of degraded buried piping. A risk-informed approach was taken to evaluate the most common aging effects in buried piping, which consists of general wall thinning and localized loss of material/pitting. Degradation over time was included in the methodology development. Fragility modeling procedures for degraded buried piping were developed and the effect of degradation on fragility and core damage frequency (CDF) was determined. The development of DAC considered the effects of degradation over time so that the number of years required for the buried pipe to reach a level of degradation that represents a potentially significant plant risk can be determined. NUREG/CR-6876 included necessary conditions for the usage of DAC, including the types of buried piping systems, configurations, materials, applicable pipe loads (e.g., pressure, surcharge, live load, etc.) and other conditions. It was recognized in this study that seismic induced stresses in buried piping are self-limiting since deformations or strains are limited by seismic motion of the surrounding media. DAC and related methodology developed in this report were intended to provide guidance to the NRC staff for making timely assessment whether observed degraded conditions potentially have an immediate significant effect on plant risk. However, as is the case in the concrete study, the DAC are not intended for industry applications as a tool to justify existing degraded conditions. 



\section{LICENSEE EVENT REPORTS}

Licensee event reports (LERs) are one of the first sources utilized for collecting and reviewing age-related degradation occurrences of structures and passive components (SPCs) at nuclear power plants (NPPs) in the U.S. LERs, which are governed by 10 CFR 50.73, are considered to be a consistent and complete source of information for obtaining degradation records. This section describes the list of SPCs in more detail, methods used to identify and retrieve LERs, development of an LER review assistance program, summary of results, and trending analysis. Reviews and findings using other sources are documented in Section 4 of this report.

\subsection{Structure and Passive Component List}

Based on the discussions between BNL and KAERI, a list of structures and passive components (SPCs) introduced in Section 1 were established as a combination of a list of structure and components provided by KAERI and a list of 18 components reported in NUREG/CR-6679. Eight component categories from NUREG/CR-6679, namely the cable tray systems, conduit systems, cooling tower, electrical conductors, HVAC duct, insulation/seal, structural seismic gap, and water-control structures, are not included in this search effort because they are not considered to be risk significant according to the component list provided by KAERI.

This section describes the selected SPCs in more detail by including their subcomponents and the information relevant to later discussion in this report.

\section{Anchorage}

Anchorage covers all structural components that serve as a connection between a concrete element and a piece of equipment. Anchorages include embedded anchors, expansion anchors, grout (used beneath baseplates), and steel embedments.

\section{Concrete}

The concrete category includes reinforced concrete buildings, water intake structures, pump house, underground structures, concrete walls/floors/ceilings/mats/foundations, canals, fuel pools, pits, pedestals, prestressed concrete structures, manholes. It also includes masonry walls and block walls.

\section{Containment}

Containment is a special type of structure in nuclear power plants used as a final barrier to prevent the release of radioactive materials to the environment following a postulated accident that may occur inside the containment. Therefore, the containment is not categorized as either concrete or structural steel components. The containment category includes the steel shell or concrete shell, prestressing system if applicable, penetrations, torus (if applicable), bellows, liners, and supports. The prestressing system includes tendons, tendon anchorages, and grease used in the tendon conduits to prevent degradation of the tendons.

\section{Exchanger}

The exchanger category includes steam generator, heat exchanger, condenser (including ice condensers used in the design of some US plants), and supports.

\section{Filter}

Filters include mechanical \& HVAC - screens, separators, strainers, absorbers, relevant supports, and housings. It should be noted that the subcomponents for filters do not include regular maintenance items which are examined or replaced on a regular basis. 


\section{Piping system}

Piping systems include piping, underground piping, fittings, small bore piping \& tubing, sleeves, and pipe supports. Hydraulic or mechanical assemblies of snubbers are not considered since they are active components and subject to periodic inspection and maintenance if needed.

\section{RPV}

Subcomponents of reactor pressure vessels (RPVs) include the shell, internals, passive components for control rod drive mechanisms (CRDMs), and relevant supports. Although pressurized, RPVs are distinguished as an individual component from other vessels because of their unique and important roles in nuclear power plants.

\section{Structural steel}

The structural steel category includes steel frames, trusses, platforms, supports, bolts, nuts, studs, fasteners, liners, doors, covers, hatches, and support to all types of equipment.

\section{Tank}

Tanks are those vessels that are subject only to atmospheric pressure.

\section{Vessel}

Vessels are pressurized, and include subcomponents pressurizer, other pressurized vessels, and their supports.

Degradation occurrences included in this report do not necessarily correlate to the number of degraded elements at a specific plant and date. If several degraded elements are found at the same plant and the same date, they are grouped into one degradation occurrence if they are the same subcomponent.

It should be noted that the subcomponents in some of these component categories are not always the same as those used for NUREG/CR-6679. In particular, the penetrations under the containment category and the piping, fittings, small bore piping, tubing, and sleeves under the piping system category, were removed from further review (after some initial tabulation in NUREG/CR-6679) because they were being addressed by other existing NRC programs. In addition, the 105 degraded piping system components reported in NUREG/CR-6679 were only a part of all degradations that actually occurred, because the total number of piping system degradation occurrences were found to be too numerous. For this research, the counting of the degradation occurrences using LERs was more thorough in the sense that none was intentionally discarded. These differences were taken into account in the statistical comparison and the trending analysis.

For each component where degradation was identified, a degradation occurrence record (DOR) was developed. The DOR includes the following fields in the database: ML number, LER reference number, Event Date, Plant Name, Docket Number, System, Component, Subcomponent, Aging Effects, Aging Mechanisms, How Identified, Evaluation Method, and Repair method. These fields are explained in the subsections below.

\subsection{Licensee Event Reports}

Since this research must be based on publicly available information, LERs were chosen to be one of the primary sources for review. The LERs were also selected as the primary source for the following reasons: standardized requirement in accordance with 10 CFR 50.73, continuous in time, completeness of information, good coverage of significant events, well formatted, and easy accessibility. 
Since January 1, 1984, an LER is required by 10 CFR 50.73 to be submitted within 60 days after the occurrence of a significant event, following an immediate notification (required by $10 \mathrm{CFR}$ 50.72). Each LER and its revisions are generally required to address specific events and plant conditions. Unrelated events or conditions, including cases of the same or similar components but different causes or separate events/activities, should be reported in different LERs. Voluntary LERs are encouraged to be submitted even if the events are not required by 10 CFR 50.73 and other requirements, but are believed by the licensee to be safety significant. An LER may be canceled by letter provided that the cancellation has a sound and logical basis.

LERs have been used by the NRC staff to study potentially generic safety problems, and assess trends and patterns of operational experience, as well as in other applications [NUREG-1022]. As specifically required by 10 CFR 50.73, a licensee shall report the following items when writing LERs:

1. Plant shutdown by technical specifications

2. Operation or condition prohibited by technical specifications

3. Deviation from technical specifications under 10 CFR 50.54 (x)

4. Degraded or unanalyzed condition

5. External threat or hampering

6. System actuation

7. Event or condition that could have prevented fulfillment of a safety function

8. Common-cause inoperability of independent trains or channels

9. Radioactive release

10. Internal threat or hampering

11. Transport of a contaminated person offsite

12. News release or notification of other government agency

13. Loss of emergency preparedness capabilities

14. Single cause that could have prevented fulfillment of the safety functions of trains or channels in different systems

For each of these 14 reportable items, NUREG-1022 provides detailed description, discussion, and many application examples.

LERs are also required by 10 CFR 50.73 to contain a brief abstract; a clear, specific, and narrative description of the event; an assessment of the safety consequences and implications of the event; corrective actions; and references to any previous similar events. In addition, LERs start with a signed cover letter and usually have a very instructive title. Most LERs utilize NRC FORMs 366/366A/366B, which include information such as facility name, docket number, event date, LER number, report date, etc. Compared to other sources of information as described in NUREG/CR-6679, LERs have the advantage of information completeness and rule-based format, which can facilitate automation in their processing.

\subsection{Keyword Search Approach through NRC ADAMS System}

The LERs used in NUREG/CR-6679 were processed through the Sequence Coding \& Search System (SCSS) that was created and maintained for the NRC by the Nuclear Operations Analysis Center at the Oak Ridge National Laboratory (ORNL). This database system contains over 35,000 LERs from 1980 to 1997, of which NUREG/CR-6679 covered the period of 1985 to 1997. 
SCSS provides a searchable way to process the LERs. However, this system is not accessible anymore which precludes its use in this search effort. Development of a system similar to SCSS would require a sizable effort beyond the available resources for this project. Therefore, the identification and review process of recent LERs will be based on the NRC ADAMS system.

\subsubsection{NRC ADAMS System}

Quoting from the NRC website, "The Agencywide Documents Access and Management System (ADAMS) is an information system that provides access to all image and text documents that the NRC has made public since November 1, 1999, as well as bibliographic records (some with abstracts and full text) that the NRC made public before November 1999. The NRC continues to add several hundred new documents daily. ADAMS permits full-text searching and enables users to view document images, download files, and print locally."

More specifically, public documents since November 1, 1999 are provided in the NRC ADAMS system as image and text files through the publicly available records system (PARS); public documents before that date are mostly provided only as bibliographic records through the public legacy library (PLL). Web-based access and Citrix-based access are the two methods for using ADAMS, the former with a web-based search engine for documents after November 1999 and the latter with the downloadable Citrix software. Excellent help documents are provided on the NRC website for usage of these two methods. Because PLL does not include full text for all records and does not include recent records, web-based ADAMS access, i.e., PARS, was chosen in this research. Moreover, NUREG/CR-6679 covered LERs between 1985 and 1997; utilization of PARS fortunately extended that period after 1999 with just 1998 omitted. However, since the search of degradation occurrences aims at identifying any trend shift, omitting one year does not statistically affect the conclusion.

The NRC ADAMS system features simple and advanced search methods, additionally with a full range of sorting and other result representation options. It has three query methods, namely Concept, Boolean, and Pattern modes. Detailed instruction on the usage of these modes and comparisons of their advantages are available through the ADAMS help documents. The search results can be further filtered and refined to obtain records that are more relevant. In ADAMS system, any of its documents can be identified by a unique accession number (MLxxxxxxxx).

\subsubsection{Development of the Keyword Search Approach}

Using the advanced search method with "LER" as the document type field, a total of 4323 LERs had been found through PARS, as of 04/16/2008 (many new documents including new LERs are constantly collected in the ADAMS system). Since the number of LERs is too large to be reviewed with the allowable resources, various query methods and associated options were initially explored to identify an appropriate method to process the LERs for identification of degradation occurrences of the SPCs. This subsection is a brief summary of the development of the keyword search approach, which serves as the justification of the applicability of this method.

All three query modes of the ADAMS system were evaluated to identify the best search strategy. Both the Concept mode and the Boolean mode returned virtually the entire set of LERs using an extensive list of aging/degradation related keywords (with appropriate Boolean operators), such as: aging, degradation, deterioration, corrosion, cracking, failure, spalling, rupture, peeling, thinning, "loss of material," "excessive deformation," wear, "loss of preload," "stress relaxation," "water accumulation," fouling, plugging, loosening, leaking, wear, pwscc, scc, igscc, iga, tgscc, odscc, hsc, embrittlement, organism, erosion, vibration, "chemical attack", fatigue, and rust. The use of quotes was required when using multiple words within a single phrase. 
Pattern search had many unrelated expansion of the original search terms, and therefore, was judged no better than the previous two modes. Therefore, the decision was made to develop keywords for each of the 10 component categories. In addition, the exploration also showed that the keywords in the "Filter with" field appeared to be an appropriate approach.

The options for using the ADAMS system were chosen to be:

Filter With Field: keywords with Boolean operations

Document Type: LER

Number of Docs to Retrieve: 99999

Number of Docs to Display: 99999

Sort by: Document Date in Descending Order

The result fields for the returned document records include: Title, Accession Number (ML \#), Docket Number, Document Number, Estimated Page Count, and Document Summary.

By trial and error, the keywords used in the "Filter With" field for the Anchorage were selected to be anchor* or grout or "steel embedment." The use of the star character permits retrieval of various forms of the word anchor such as anchors, anchorage, anchored, or anchoring. Using this keyword set, ADAMS returned 80 LERs, which were judged to be a reasonable number that can be reviewed within the project resource limit provided other component categories would return similar number of LERs. The degree of coverage of these 80 LERs over the entire potential degradation occurrences of anchorages were not known.

Similarly, using a keyword set of concrete or masonry or rebar, 99 LERs were returned by the ADAMS system for the concrete component category. However, this keyword list was not considered to cover the entire population of this category in the ADAMS database. For example, more than 2000 LERs were returned using a much more rigorous enumeration of the possible subcomponents, which were formed as a Boolean expression: (concrete or intake or pumphouse or underground or mat or mats or foundation or canal or pit or pedestal or prestress or manhole or masonry or block or pool) and (crack or degradation or aging or deterioration or corrosion or failure or spalling or rupture or peeling or wear or preload or relaxation or fouling or plugging or leaking or embrittle or erosion or chemical or attack or fatigue or rust).

From the trial results for the anchorage and the concrete component categories, there were two issues in this keyword approach: (1) the number of returned LERs and (2) the degree of coverage of the component population. One may get a reasonable number of LERs to process but leave the coverage in question, or achieve a high confidence in population coverage but retrieve too many LERs that cannot be processed within the resources available to perform this research study. Another problem is that some LERs may be returned for more than one component categories and therefore will need to be reviewed multiple times. The resultant total number of LERs reviewed may be even larger than the total number of LERs (4323) for a fair coverage of the population. In summary, this keyword approach appeared to be an art of balance in creating an appropriate list of keywords: it can either (1) reduce the number of returned LERs to a reasonable level for processing but with the potentially degraded coverage or, (2) increase the coverage level of the population but with too many returned LERs to be processed. In the meantime, developing the keyword list required repetitive accesses of the ADAMS system to check the returned LER list and represented a sizable effort.

Therefore, the development of the keyword search approach was determined to be inefficient and was stopped after the concrete component category. Accordingly, all LERs after 1999 were 
reviewed individually so that a full coverage was achieved and any LER was avoided being reviewed more than once.

\subsection{Development of LER Reading Assistance Tool}

\subsubsection{Summary of the Development Process}

The initial approach for processing every individual LER is first to retrieve all LERs, sorted by document date in descending order, through the ADAMS system and then to review them in that order. This approach required bookkeeping each current LER being reviewed and retrieving the list of all LERs after each interruption such as computer shutdown at the end of each day, work breaks during the day, and internet/web server issues. On average, each LER has about five to six pages, with the longest being 311 pages. After an initial experiment for the first a day or so, it was estimated that each LER requires about 5 minutes to review which is equivalent to reviewing $96 \mathrm{LERs} /$ day. The total estimated effort could be 45 days, which would result in no time for database development, statistical analysis, and annual report preparation. This difficulty led to the development of computerized tools to assist the LER review.

Since the NRC ADAMS system may not be accessible at all time, for example, the ADAMS server may be down or the internet connection may be lost unexpectedly, all LERs were downloaded at once and the review was carried out locally. Only the text files (in HTML format) were downloaded in order to facilitate the computer assistance in processing and to avoid the unnecessary download time and consuming a large amount of disk space if the image files were downloaded. The actual retrieval of the 4323 HTML files utilizes an in-house web crawler, which was developed in Python (computer language) and was capable to automatically login the ADAMS system, submit the advanced search form, and retrieve the HTML files. There were a small number of LERs that could not be downloaded automatically and were logged by the spider for manual download. The entire retrieval process took about three hours and the downloaded files consumed $116 \mathrm{MB}$ of disk space.

As the HTML files include a lot of tags that are not meaningful, the HTML files are then translated into pure text files by trimming out these tags and some other irrelevant text. The resulting text files have a total size of $73 \mathrm{MB}$ on a disk.

Figure 3-1 through Figure 3-4 show a sample LER in text format, showing its beginning part, abstract part, cause part, and action part, respectively. These various parts are common for most LERs as required by the 10 CFR 50.73, and are used to speed up the review of these LERs. In contrast, Figure 3-5 shows the image of the same LER, with the NRC form 366 that shows the abstract part.

Compared to image files (pdf or tiff format), text files have the advantages of being: locally available; easy for computers to process; relatively easy to decode the LER number, event date, plant name, and docket number from these text LERs; and easy to locate the four helpful sections in the LERs (cause, title, abstract, and corrective action). However, the text files are no better than the image files than the pdf files to human readers. In addition, a small number of LERs are only available in image format. The goal of developing an assistance tool for LER processing was to (1) automatically decode relevant information such as LER number, event date, plant name, and docket number, (2) automatically color-code the four sections, (3) automatically identify the plant type, (4) automatically color-code the entire text such that reading of the LER is more effective, and (5) manage the saved degradation occurrences. This tool can effectively process all text LERs and can retrieve an image file from the NRC ADAMS system on rare cases where a text LER is not available locally or not sufficient to make a decision. 
Because of our extensive experience in developing Python programs, the initial development of the assistance tool, so called LER Reader, required only an effort of 4 days (including the development of the web crawler); later improvement of the LER Reader involved more time during the processing of the LERs. This development ended up with 1009 lines of hand written codes and thousands of lines of generated codes. It should be noted that the improvement of the tools does not require a reprocessing of the reviewed LERs because the LER Reader only assists us to review but not to make the decisions. The improvement was intended to increase the review speed not to lower the quality of the review.

\subsubsection{Introduction of the LER Reader GUI}

Figure 3-6 shows the annotated graphical user interface (GUI) of the LER Reader. The large box labeled as "LER Text" on the right hand side is used to display the parsed LER texts; while those tables, boxes, and buttons on the left hand side provide the controlling functionalities. The table on the upper-left corner labeled as "Degradation Occurrence Record (DOR)" is used to enter the DORs either automatically by the LER Reader or manually, depending on the fields.

Tool Set One includes buttons and a text box to control the process of LERs. Clicking "Reload Terms" reload the component list, system list, subcomponent list, aging effects list, and aging mechanism list, each of which is stored in one text file. Button "Save" is for saving the DOR filled in the table. The text box with "- 1 " indicates the current LER id within the range of 0 to 4322. Button "Next ML" advances to next LER and triggers the auto parsing of the new LER. Buttons "I" and "O" can be used to zoom in or out the LER text, i.e., to increase or decrease the font size for the LER text. Button ">Pdf" can be used to retrieve from the NRC website and show the corresponding pdf file as needed for rare cases. The four buttons "> Title", ">Abstract", ">Cause", and ">Action" are intended to jump in the "LER Text" box to the corresponding section of the LER text. A few convenient short cut commands for these navigation functions are also available by pressing the right mouse button. Pressing the middle mouse button reformats the text for better readability.

The text box labeled as "LER Title and Plant Type" shows the title of the LER and the decoded plant type, which can be "PWR", "BWR", or "???". A plant type of "???" indicates either this LER is not about any of the 104 NPPs in the US or the LER Reader cannot determine the plant type.

The text box labeled as "Saved Recorded Statistics and Other Information" is used to show various information or result of the LER Reader. Figure 3-7 shows the DOR statistics shown in this box.

Tool Set Two includes a status label for the number of save DORs and two buttons for control of the display of saved DORs in the text box below. The button "Refresh" is required to click if the user wants to browse through the saved DORs; the simple summary of the saved DORs will be shown in the text box below after this button is clicked. Clicking "Next Saved LER" iterates through the saved DORs.

\subsubsection{Application of the LER Reader}

Upon loading an LER by clicking the "Next ML" button, the LER Reader does the following steps: 
1. Parse the LER to obtain the LER Reference number, event date, plant name, and the docket number; unsuccessful items will appear as empty in the DOR table.

2. Parse the LER to locate the positions of the Title, Abstract, Cause, and Corrective Action; unsuccessful items will have a default value 0 , which is the start of the text LER.

3. Jump to the Cause section in the "LER Text" box, which may show the beginning of the LER if the parsing of the Cause section failed in step 2.

4. Check if the DOR was already saved in the database, which may use a revision of the current LER; a warning dialog will show if an existing DOR is found.

5. Show the title and the plant type.

6. Colorize the LER text; yellow suggests the LER is about something not related to degradation of SPCs while red suggests it is related to degradation.

The procedure to review an LER starts with loading an LER by clicking the "Next ML" button. Upon successful loading, one may read the entire LER for identification of any degradation occurrences. However, the utilization of the LER Reader to speed up the review process requires the following suggested steps:

1. Advance to next LER if a dialog shows a DOR existed in the database.

2. Read the title and the plant type; if the plant type is "???", the LER is most likely not related to the NPP. The title is usually very instructive.

3. Read the Cause section and other sections if needed for any recordable DOR; click the right mouse button to iterate through the four sections in the order of Cause, Title, Abstract, and Corrective Actions; or scroll through the whole LER.

4. If the LER text is not meaningful, try to directly load the PDF file from the NRC website by clicking ">Pdf", or by visiting the NRC ADAMS system directly with the ML number. Copying the HTML content from the NRC website to the "LER Text" box will trigger the LER Reader to parse and save it automatically.

Figure 3-8 through Figure 3-16 show various use scenarios of the LER Reader. It is obvious that colorizing LERs as described previously greatly increases the absorption of information for the users. The nature of the event can be determined by looking at the highlighted words or phrases, after a brief experience of the colorized LERs.

For the majority of cases, the correct LER reference number, event date, plant name, and docket number can be determined automatically by the LER Reader. There is no need for the user to identify this information in the LER and then enter these values into the table. The LER Reader can appropriately colorize the relevant words and phrases and can correctly identify all or most of the four important sections. The reviewer usually does not need to scroll through the whole LER to find all the necessary information.

The combination of the LER Reader and the associated LER text files saved a lot of manual accesses to the ADAMS system and avoided the time consuming download of the image files. There was also no need to carefully maintain the order of the 4323 LERs and to keep track of the current LER after resuming from an interruption.

During review of the LERs, all DORs were saved to a text file, which later can be imported into an Microsoft (MS) Access database for statistical analysis.

Compared to the estimated speed of reviewing 96 LERs/day for the approach that utilizes the NRC ADAMS system directly, review of 200 to 300 LERs/day was achieved on average using the LER Reader approach. The total amount of time including the program development was 
about half of the original estimate of 45 days. As the experience in using the LER Reader and in reviewing LERs grew, the process of identifying and tabulating applicable LERs became faster. Indeed, more than 350 LERs were processed on the last day. Had more LERs been available for processing, the saving by the LER Reader approach would be more significant. In addition, the use of the LER Reader ensures much greater accuracy since much of the data was automatically transcribed into the database once the user noted the applicability of the LER.

\subsection{Results Assessment}

\subsubsection{Database Integration}

The text file of DORs saved by the LER Reader were imported as the DOR table to an MS Access database, which include the fields: ML number, LER Reference number, Event Date, Plant Name, Docket Number, System, Component, Subcomponent, Aging Effects, Aging Mechanisms, How Identified, Evaluation Method, and Repair method.

During the trending analysis to be presented later in this report, there was a need to compute the plant age when a particular degradation event occurred. To this end, the date that the construction permit (CP) was issued was selected as the starting time of the plants. The CP date and the operation license (OL) date were obtained from the U.S. NRC 2007-2008 Information Digest, Appendix A [NUREG-1350, 2007]. A database table was then created for all 104 operating NPPs in the U.S. by incorporating the plant information from the NRC website and these CP and OL dates. The fields of this table include plant name, docket number, reactor type, location, owner/operator, NRC region, $\mathrm{CP}$ issued, and OL issued, as shown in Table 3-1. The NRC regions showing the NPP distribution in the U.S. are shown in Figure 3-17.

The DOR table and the U.S. plant information table are related by the docket numbers. Additional queries, reports, forms, macros, and pages were created to assist the database integration and statistical analysis. In order to perform the trending analysis, the database used in NUREG/CR-6679 was updated to include the plant information table. A number of PivotTables in MS Excel were utilized to connect to the databases and to generate data during the statistical analysis.

\subsubsection{DOR Assessment}

DORs are summarized in Table 3-2 and Table 3-3, presenting the primary information and the secondary information for the DORs, respectively. The two tables are one-to-one correlated by the field ID. The primary information table includes fields ID, component, subcomponent, system, aging effects, aging mechanics, plant and event date, which are significant in describing a DOR. The secondary information table provides supplemental information for the DORs, which consists of how identified, evaluation method, repair method, docket number, LER reference number, and ML number, and also includes ID, component, and subcomponent for easy identification. DORs in the primary information table are in the alphabetical order of the fields component, subcomponent, and system, which together identify the specific SPCs. DORs in the secondary information table are in the ascending order of ID. An entry of "NA" in these tables signifies that there was no or insufficient information available in the LER for that entry. Although a docket number can be used to uniquely identify a plant or a particular unit in a plant if there are multiple units at the site, the plant name and the unit number are still provided in these tables for convenient reference. Table 3-4 shows the abbreviations used for the aging mechanism field. 
In the DOR tables, there are three entries under an additional component category "other", which includes those DORs that were determined not suitable for any of the 10 SPC categories. Excluding these three entries, A total of 223 DORs were identified from the LERs for the period of 1999 to the present (4/16/2008), including just a few from 1998 as well.

Figure 3-18 shows the distribution of the SPC degradation occurrences by component categories, with the number of the degradation occurrences annotated over the bars. As would be expected, the piping systems have the most degradation occurrences reported in the LERs, about $36 \%$ of the total DORs. Exchangers and RPVs have the next two largest number of degradation occurrences, representing about $22 \%$ and $17 \%$ of the total DORs, respectively. The other seven component categories represent less than $25 \%$ of the total DORs; they are vessels, filters, containments, structural steel, tank, concrete, and anchorage in descending order of the number of degradation occurrences. It should be noted that the number of degradation occurrences for a particular category is also a function of the quantity of components in that category that is present at a plant. So for example, the number of degradation occurrences for piping was expected to be large, because there are many piping systems at a NPP and many of these piping systems can be quite long.

The total number of DORs for structural type components, i.e., containments, structural steel, concrete, and anchorages, is only 18 , about $8 \%$ of all DORs. However, this does not necessarily indicate that there have been fewer degradations occurring in these structures. Rather, it is because of the nature of the structural degradation and the nature of LER reporting requirement that are judged to result in fewer instances of degradation. As described previously, LERs report any degradation situations that seriously affect the plant safety or result in any unanalyzed conditions that could significantly compromise the plant safety. The events reported in LERs are often from operating experiences. Structural degradations usually have less immediate impact on plant safety, and therefore, are less likely to be observed and reported in LERs. However, structural degradations can be significant risk factors to plant safety when a severe environmental event, for example, a large earthquake, occurs. Most structural degradations can be found in literatures that involved results from special inspection efforts. For example, NUREG-1522 covers data obtained from walkdowns conducted at six older vintage plants [NUREG-1522, 1995].

Other distributions over various measures are provided in the next subsection, with comparisons to data reported in NUREG/CR-6679.

\subsection{Trending Analysis}

To evaluate the possible trends in degradation occurrence data, the DORs in the past decade (approximately 1999 to 2008 collected in this study) and those reported in NUREG/CR-6679 (approximately 1985 to 1997) were compared. In order to make a sound comparison, a few differences between the current data collection using the recent LERs and those reported in NUREG/CR-6679 should be noted. These are highlighted in the following:

1. Difference in Information Sources: unlike the current set of data collected in this study which rely on LERs 1999-2008, NUREG/CR-6679 covered a larger set of information sources, which included LERs 1985-1997, NUREGs, NRC/IE Information notices, Correspondences, Generic Letters, NRC Bulletins, IR Circulations, SECY documents, and other publicly available documents.

2. Difference in LER retrieval methods: one-by-one evaluation for LER 1999-2008 versus computer based search for LER 1985-1997 through SCSS at ORNL. 
3. Difference in component lists: 10 component categories for current collection versus 18 component categories for NUREG/CR-6679.

4. Difference in subcomponent lists: all SPCs for current collection versus reduced scope for NUREG/CR-6679 (e.g., piping, fittings, small bore piping \& tubing, sleeves, penetrations, etc.).

These differences were considered in the comparisons presented in the trending analysis. Hereafter, the various comparisons utilize either the original data from NUREG/CR-6679 that includes all sources or only those related to LER 1985-1997 for consistency with the data collected in this research. The corresponding labels in the figures to be introduced are selfexplained regarding their information sources.

Figure 3-19 shows the distributions of the degradation occurrences by components for three series of data: LER 1999-2008, NUREG/CR-6679, and LER 1985-1997, respectively. The bar chart is in the same order as reported in NUREG/CR-6679. Similarly, Figure 3-20 shows a normalized version of the same figure with the total numbers of DORS in each series as the basis. Considering LER 1999-2008 and NUREG/CR-6679, exchangers, piping system, and RPVs are the first three categories with the greatest number degradation occurrences. Since the piping system DORs for LER 1985-1997 were determined to be very large and did not include all of the occurrences in the SCSS database, the actual number of DORs of piping system for LER 19851997 are artificially low. These figures also show that filters were the second largest category using LER 1985-1997. Both LER data series confirm the observation in the previous section that LERs do not report many structural DORs, especially containment, concrete, and anchorage.

Figure 3-21 shows the distribution comparison of the SPC degradation occurrences over time, with the top figure showing the two series from NUREG/CR-6679 and with the bottom figure showing the series representing LER 1999-2008. First of all, the strong correlation over the years between the two series from NUREG/CR-6679 indicates that LER 1985-1997 represent a significant portion of the NUREG/CR-6679 data. Regardless of the partial years 1997, 1998, and 2008 , the yearly DORs varies somewhat in cycles, which might correspond to inspection intervals that often are scheduled at refueling or are required by special NRC mandatory inspection requirements. On a yearly basis, there appear to be slightly more DORS from LER 1998-2008 than from LER 1985-1997. This observation may be due to the difference in reviewing LERs; the computer search approach for LER 1985-1997 may not have the same level of coverage of the degradation population as the one-by-one review approach for LER 1999-2008.

Excluding the three partial years, DORs from LER 1985-1996 and LER 1999-2007 are correlated to their plant ages at event (PAAE), which is defined as (Event Date - CP Issued Date). Table 3-5 and Table 3-6 show the number of DORs and the number of NPP units that these DORs belong to as a function of PAAE, for LER 1999-2007 and LER 1985-1996, respectively. Taking the row of a PAAE equal to 33 as an example, it shows that there were 20 NPP units which had 30 DORs at the time that these units were 33 years old as defined by PAAE. The fourth columns in these two tables list the average number of DORs that were obtained by dividing the number of DORs by the number of affected NPP units.

Figure 3-22 visualizes the number of DORs and the number of associated NPP units distributed over the PAAE. For both data series LER 1985-1996 and LER 1999-2007, the distributions show central peaks at 24 years and 33 years (PAAE), respectively, on curves related to DOR distribution and NPP unit distribution. These peaks and the hill shape distributions may suggest the distribution of construction time of the U.S. NPP population. As the two data series are approximately apart by 10 years, the associated curves show similar shifts, in particular with the 
peaks apart by 9 years. It is important to note that the upward shift between the two series shows the increase of the numbers of DORs and the affected units, indicating increasing age-related degradations. The peaks increase from 22 to 30 (36\%) for the number of DORs and from 15 to $20(33 \%)$ for the number of affected NPP units. The overall shift in the hill shape appears to represent linear increases in the numbers of DORs and affected NPP units. It should be noticed that the smaller numbers of DORs and affected NPP units at higher PAAE than the peak PAAE do not indicate there were less degradation for older plants, rather there were a smaller number of older plants in the operating NPP fleet. A similar observation occurs for the lower PAAE because there were fewer relatively younger plants during the 1999-2007 review period.

Figure 3-23 shows the numbers of DORs and the associated NPP units with the two data series combined. The combination was achieved by adding the related numbers for the overlapped PAAEs. The trend lines considered data up to a PAAE of 36 years, which was selected because shortly after this PAAE, the number of DORs and plants drops off rapidly which if included would skew the resulting trend lines. The trend lines show that both the number of DORs and the numbers of associated NPP units increase as PAAE increases. In addition, the increase in the number of DORs rises faster than the increase in the number of NPP units.

Figure 3-24 shows the relation of the average degradation occurrences with PAAE. As an example, the peak point in this figure shows that for plants at an age of 34 years, about 1.8 DORs per plant were reported in LERs. The dotted line designates the series LER 1985-1996 while the solid line represents the series LER 1999-2007. It appears that the average number of DORs per plant increase as the plant gets older, with a slightly higher rate for older plants as shown by the steeper slope using LER 1999-2007. The slightly higher degradation rate using more recent LERs reflects the fact that older plants show in general more degradation occurrences, and may reflect as well the lowered coverage of the possible degradation population by limiting the number of DORs in NUREG/CR-6679 (e.g. piping system) and potentially by using the computerize search approach for LER 1985-1997. By literally reading the two trend lines, the older plants (using LER 1999-2007) appear to have about 3 times more average DORs than the younger plants (using LER 1985-1997); however, this observation may not fully represent the real situation because of the differences in processing the two LER series. In addition, this observation may not be true for structural type components, as the data from LERs are less representative of the structural components. The variations in these two curves are judged to be relatively large due to the fact that these degradations are rare events and the number of DORs is relatively small.

Figure 3-25 shows the relationship of the average DORs per plant to the PAAE using the combined data series. The trend line in Figure 3-25 shows that combining the two series results in a slightly increasing average degradations per plant over PAAE and is essentially an average of the prior two trend lines shown in Figure 3-24.

Figure 3-26 shows the comparison for the distributions of the SPC degradation occurrences with respect to major aging effects among the three data series for steel components (more precisely metal components). Cracking is the most predominant aging effect for all three data series. Failure is the second most significant aging effect for LER 1998-2008 because it includes a number of aging effects that do not fall into any listed categories. DORs with aging effects of loss of material and wall thinning appear to be obtained from information sources other than LERs.

Figure 3-27 shows the comparison of the distributions of the SPC degradation occurrences by major aging mechanisms among the three data series. SCC is the most significant aging 
mechanism for all data series. Compared to the two NUREG/CR-6679 data series, LER 19992008 shows a large DOR contribution from fatigue, which is the second most significant degradation for this series. This may be due to the possibility that some components are approaching their fatigue life as NPPs get older. Moisture, organisms, chemical attack, and foreign object are shown to be less important mechanisms for LER 1998-2008; lessons learned from the past may have helped to avoid such aging mechanisms.

Figure 3-28 shows the comparison of the distributions of the SPC degradation occurrences by cracking type among the three data series. Primary water stress corrosion cracking (PWSCC) is the most common cracking type for both LER data series, partly indicating the preferences of the LER reporting system. On the other hand, intergranular stress corrosion cracking (IGSCC) was found to be the most common cracking type for the NUREG/CR-6679 data series, but it was a much less common cracking type for the other two LER data series. This suggests that other information sources provided those extra IGSCC DORs for the NUREG/CR-6679 data series.

Figure 3-29 shows the comparison of distributions of the SPC degradation occurrences by system types among the three data series. It is obvious using all series that the system most vulnerable to degradation is the reactor coolant system (RCS), as expected because the RCS includes many subcomponents that are constantly subjected to harsh environments such as high temperature, high pressure, high fluid velocity, boron acid, radiation, etc. 
Table 3-1 U.S. 104 Operating Nuclear Power Plants

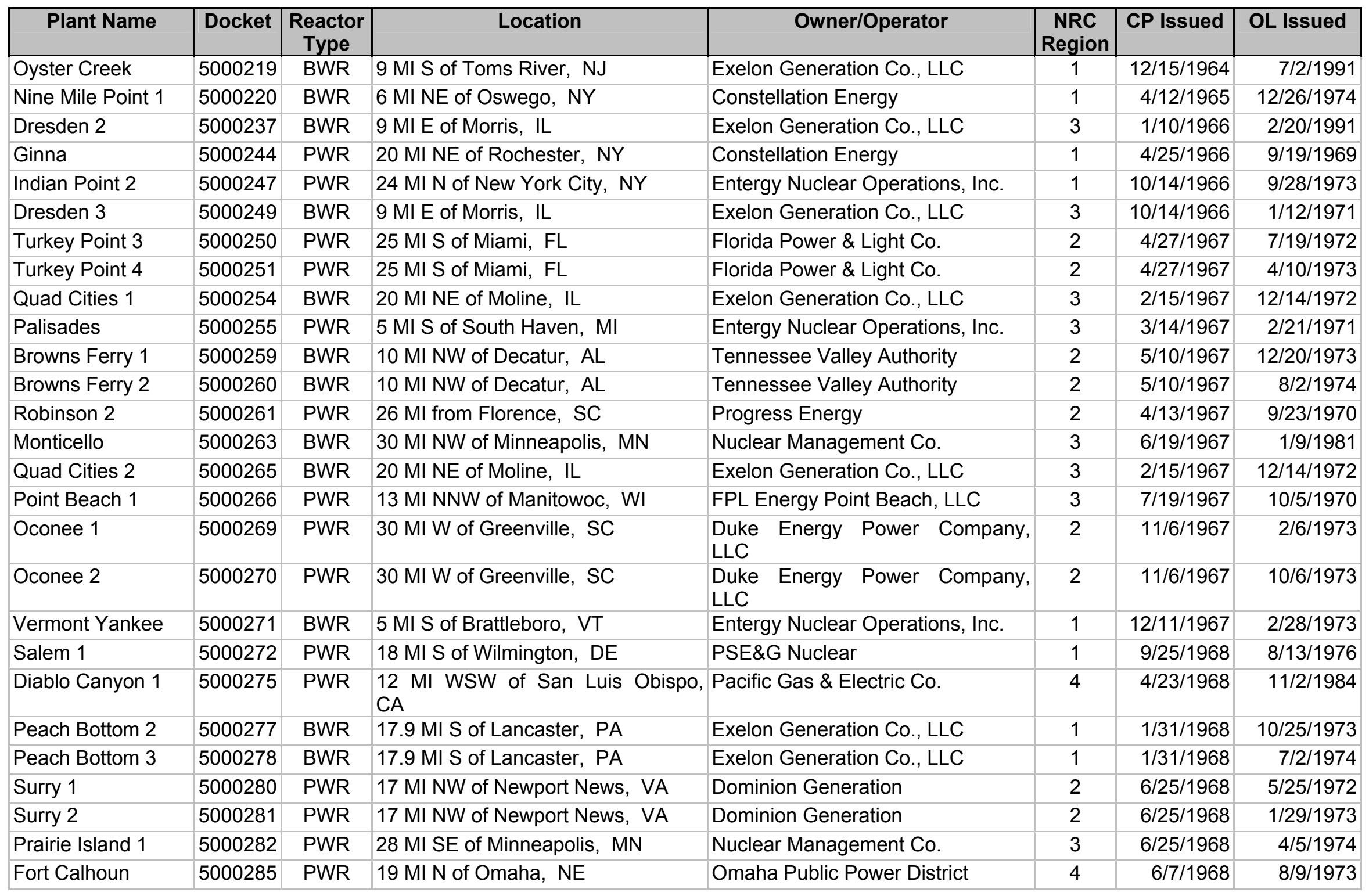




\begin{tabular}{|c|c|c|c|c|c|c|c|}
\hline Plant Name & Docket & $\begin{array}{l}\text { Reactor } \\
\text { Type }\end{array}$ & Location & Owner/Operator & \begin{tabular}{|c|} 
NRC \\
Region
\end{tabular} & CP Issued & OL Issued \\
\hline Oconee 3 & 5000287 & PWR & 30 MI W of Greenville, SC & $\begin{array}{l}\text { Duke Energy Power Company, } \\
\text { LLC }\end{array}$ & 2 & $11 / 6 / 1967$ & $7 / 19 / 1974$ \\
\hline Three Mile Island 1 & 5000289 & PWR & $10 \mathrm{MI}$ SE of Harrisburg, PA & Exelon Generation Co., LLC & 1 & $5 / 18 / 1968$ & 4/19/1974 \\
\hline Pilgrim 1 & 5000293 & BWR & 4 MI SE of Plymouth, MA & Entergy Nuclear Operations, Inc. & 1 & $8 / 26 / 1968$ & 9/15/1972 \\
\hline Browns Ferry 3 & 5000296 & BWR & $10 \mathrm{MI}$ NW of Decatur, AL & Tennessee Valley Authority & 2 & $7 / 31 / 1968$ & 8/18/1976 \\
\hline Crystal River 3 & 5000302 & PWR & $7 \mathrm{MI}$ NW of Crystal River, FL & Progress Energy & 2 & 9/25/1968 & $1 / 28 / 1977$ \\
\hline Kewaunee & 5000305 & PWR & $27 \mathrm{MI}$ E of Green Bay, WI & Dominion Generation & 3 & $8 / 6 / 1968$ & $12 / 21 / 1973$ \\
\hline Prairie Island 2 & 5000306 & PWR & $28 \mathrm{MI}$ SE of Minneapolis, MN & Nuclear Management Co. & 3 & $6 / 25 / 1968$ & $10 / 29 / 1974$ \\
\hline Salem 2 & 5000311 & PWR & $18 \mathrm{MI}$ S of Wilmington, DE & PSE\&G Nuclear & 1 & 9/25/1968 & $5 / 20 / 1981$ \\
\hline Arkansas Nuclear 1 & 5000313 & PWR & $6 \mathrm{MI}$ WNW of Russellville, AR & Entergy Nuclear Operations, Inc. & 4 & $12 / 6 / 1968$ & $5 / 21 / 1974$ \\
\hline D.C. Cook 1 & 5000315 & PWR & $11 \mathrm{MI} \mathrm{S}$ of Benton Harbor, MI & Indiana/Michigan Power Co. & 3 & $3 / 25 / 1969$ & $10 / 25 / 1974$ \\
\hline Diablo Canyon 2 & 5000323 & PWR & $\begin{array}{l}12 \text { MI WSW of San Luis Obispo, } \\
\text { CA }\end{array}$ & Pacific Gas \& Electric Co. & 4 & $12 / 9 / 1970$ & $8 / 26 / 1985$ \\
\hline Brunswick 2 & 5000324 & BWR & $2 \mathrm{MI} \mathrm{N}$ of Southport, $\mathrm{NC}$ & Progress Energy & 2 & 2/7/1970 & $12 / 27 / 1974$ \\
\hline Brunswick 1 & 5000325 & BWR & $2 \mathrm{MI} \mathrm{N}$ of Southport, $\mathrm{NC}$ & Progress Energy & 2 & 2/7/1970 & $11 / 12 / 1976$ \\
\hline Sequoyah 1 & 5000327 & PWR & 9.5 MI NE of Chattanooga, TN & Tennessee Valley Authority & 2 & $5 / 27 / 1970$ & 9/17/1980 \\
\hline Sequoyah 2 & 5000328 & PWR & 9.5 MI NE of Chattanooga, TN & Tennessee Valley Authority & 2 & $5 / 27 / 1970$ & 9/15/1981 \\
\hline Duane Arnold & 5000331 & BWR & $8 \mathrm{MI}$ NW of Cedar Rapids, IA & Florida Power \& Light Co. & 3 & $6 / 22 / 1970$ & $2 / 22 / 1974$ \\
\hline FitzPatrick & 5000333 & BWR & $8 \mathrm{MI}$ NE of Oswego, NY & Entergy Nuclear Operations, Inc. & 1 & $5 / 20 / 1970$ & $10 / 17 / 1974$ \\
\hline Beaver Valley 1 & 5000334 & PWR & $17 \mathrm{MI}$ W of McCandless, PA & FirstEnergy Nuclear Operating Co. & 1 & $6 / 26 / 1970$ & 7/2/1976 \\
\hline Saint Lucie 1 & 5000335 & PWR & 12 MI SE of Ft. Pierce, FL & Florida Power \& Light Co. & 2 & 7/1/1970 & 3/1/1976 \\
\hline
\end{tabular}




\begin{tabular}{|c|c|c|c|c|c|c|c|}
\hline Plant Name & Docket & $\begin{array}{l}\text { Reactor } \\
\text { Type }\end{array}$ & Location & Owner/Operator & \begin{tabular}{|c|} 
NRC \\
Region
\end{tabular} & CP Issued & OL Issued \\
\hline Davis-Besse & 5000346 & PWR & $21 \mathrm{MI}$ ESE of Toledo, $\mathrm{OH}$ & FirstEnergy Nuclear Operating Co. & 3 & $3 / 24 / 1971$ & $4 / 22 / 1977$ \\
\hline Limerick 1 & 5000352 & BWR & $21 \mathrm{MI}$ NW of Philadelphia, PA & Exelon Generation Co., LLC & 1 & $6 / 19 / 1974$ & 8/8/1985 \\
\hline Limerick 2 & 5000353 & BWR & $21 \mathrm{MI}$ NW of Philadelphia, PA & Exelon Generation Co., LLC & 1 & $6 / 19 / 1974$ & $8 / 25 / 1989$ \\
\hline Hope Creek 1 & 5000354 & BWR & $18 \mathrm{MI}$ SE of Wilmington, DE & PSE\&G Nuclear & 1 & $11 / 4 / 1974$ & $7 / 25 / 1986$ \\
\hline Farley 2 & 5000364 & PWR & $18 \mathrm{MI}$ SE of Dothan, AL & Southern Nuclear Operating Co. & 2 & $8 / 16 / 1972$ & 3/31/1981 \\
\hline Hatch 2 & 5000366 & BWR & $11 \mathrm{MI} N$ of Baxley, GA & $\begin{array}{l}\text { Southern Nuclear Operating Co., } \\
\text { Inc. }\end{array}$ & 2 & $12 / 27 / 1972$ & $6 / 13 / 1978$ \\
\hline Arkansas Nuclear 2 & 5000368 & PWR & $6 \mathrm{MI}$ WNW of Russellville, AR & Entergy Nuclear Operations, Inc. & 4 & $12 / 6 / 1972$ & 9/1/1978 \\
\hline McGuire 1 & 5000369 & PWR & $17 \mathrm{MIN}$ of Charlotte, $\mathrm{NC}$ & $\begin{array}{l}\text { Duke Energy Power Company, } \\
\text { LLC }\end{array}$ & 2 & 2/23/1973 & $7 / 8 / 1981$ \\
\hline Waterford 3 & 5000382 & PWR & 20 MI W of New Orleans, LA & Entergy Nuclear Operations, Inc. & 4 & $11 / 14 / 1974$ & $3 / 16 / 1985$ \\
\hline Susquehanna 1 & 5000387 & BWR & $7 \mathrm{MI}$ NE of Berwick, PA & PPL Susquehanna, LLC & 1 & $11 / 2 / 1973$ & $11 / 12 / 1982$ \\
\hline Susquehanna 2 & 5000388 & BWR & $7 \mathrm{MI}$ NE of Berwick, PA & PPL Susquehanna, LLC & 1 & $11 / 2 / 1973$ & $6 / 27 / 1984$ \\
\hline Saint Lucie 2 & 5000389 & PWR & 12 MI SE of Ft. Pierce, FL & Florida Power \& Light Co. & 2 & $5 / 2 / 1977$ & $6 / 10 / 1983$ \\
\hline Watts Bar 1 & 5000390 & PWR & $10 \mathrm{MI}$ S of Spring City, TN & Tennessee Valley Authority & 2 & $1 / 23 / 1973$ & 2/7/1996 \\
\hline Summer & 5000395 & PWR & $26 \mathrm{MI}$ NW of Columbia, SC & South Carolina Electric \& Gas Co. & 2 & $3 / 21 / 1973$ & $11 / 12 / 1982$ \\
\hline $\begin{array}{l}\text { Columbia } \\
\text { Generating Station }\end{array}$ & 5000397 & BWR & $12 \mathrm{MI}$ NW of Richland, WA & Energy Northwest & 4 & $3 / 19 / 1973$ & $4 / 13 / 1984$ \\
\hline Shearon Harris 1 & 5000400 & PWR & $20 \mathrm{MI}$ SW of Raleigh, NC & Progress Energy & 2 & $1 / 27 / 1978$ & $1 / 12 / 1987$ \\
\hline Nine Mile Point 2 & 5000410 & BWR & $6 \mathrm{MINE}$ of Oswego, NY & Constellation Energy & 1 & $6 / 24 / 1974$ & $7 / 2 / 1987$ \\
\hline Beaver Valley 2 & 5000412 & PWR & $17 \mathrm{MI} \mathrm{W}$ of McCandless, PA & FirstEnergy Nuclear Operating Co. & 1 & $5 / 3 / 1974$ & 8/14/1987 \\
\hline Catawba 1 & 5000413 & PWR & $6 \mathrm{MI}$ NW of Rock Hill, SC & $\begin{array}{l}\text { Duke Energy Power Company, } \\
\text { LLC }\end{array}$ & 2 & 8/7/1975 & $1 / 17 / 1985$ \\
\hline
\end{tabular}




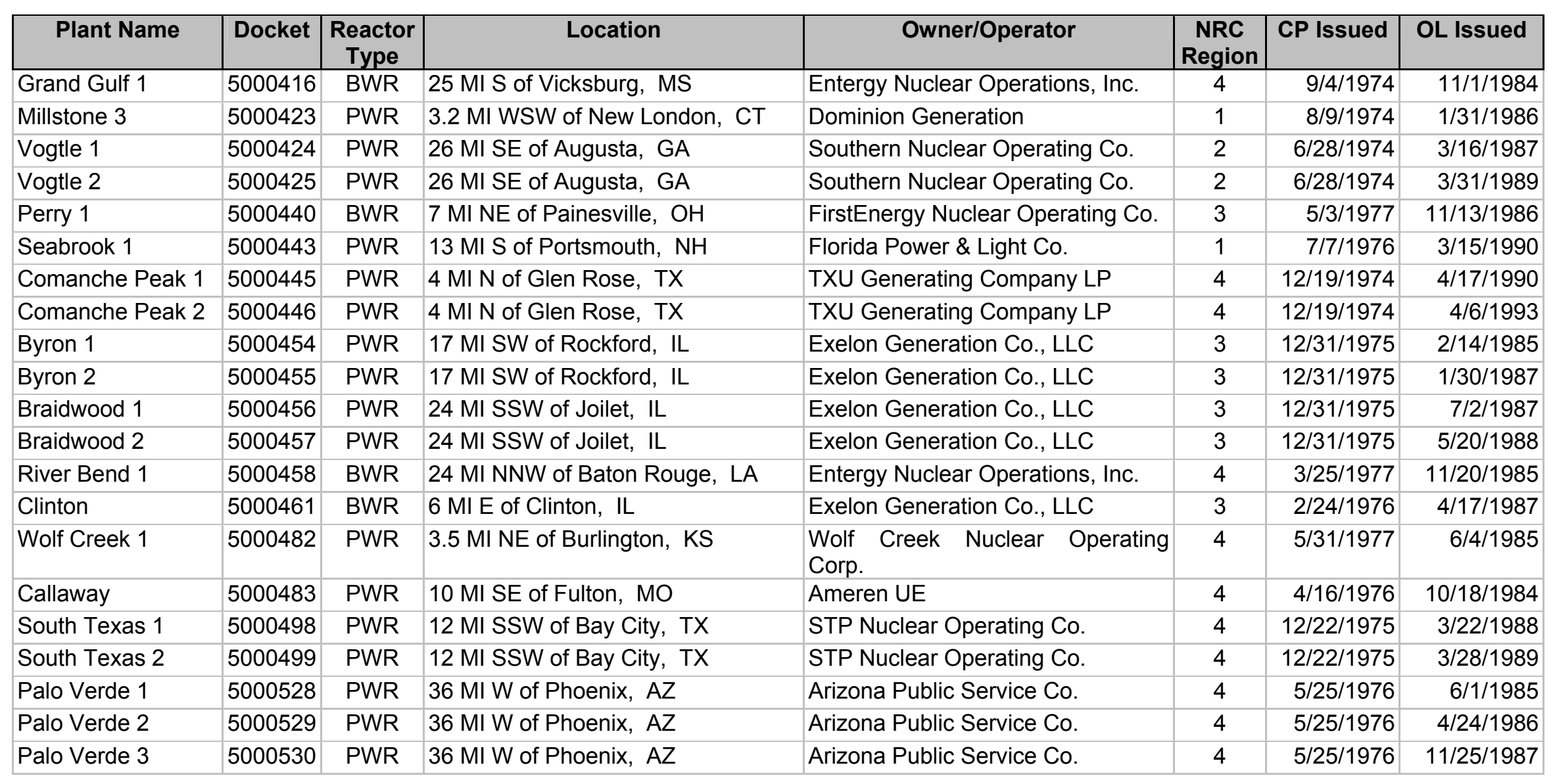


Table 3-2 Degradation Occurrence Records (Primary Information)

\begin{tabular}{|c|c|c|c|c|c|c|c|}
\hline ID & COMPONENT & SUBCOMPONENT & SYSTEM & AGING EFFECTS & AGING MECHANISMS & PLANT & $\begin{array}{l}\text { EVENT } \\
\text { DATE }\end{array}$ \\
\hline 89 & Anchorage & Nut & $\begin{array}{l}\text { RHRSW - residual heat } \\
\text { removal service water }\end{array}$ & Loss of material & Corrosion & Hatch 1 & $11 / 17 / 2003$ \\
\hline 136 & Concrete & Floor & $\begin{array}{l}\text { Radwaste building cable } \\
\text { spreading room (CSR) }\end{array}$ & Cracking & Spallation & $\begin{array}{l}\text { Columbia Generating } \\
\text { Station }\end{array}$ & $5 / 3 / 2002$ \\
\hline 203 & Concrete & Walls \& floors & Various structures & Cracking \& spalling & NA & D.C. Cook 2 & $5 / 29 / 2000$ \\
\hline 23 & Containment & CAC cooling coil / fitting & $\begin{array}{l}\text { CAC - Containment air } \\
\text { cooler }\end{array}$ & Wall thinning & Corrosion & Palisades & $1 / 19 / 2007$ \\
\hline 27 & Containment & CAC cooling coil / fitting & $\begin{array}{l}\text { CAC - Containment air } \\
\text { cooler }\end{array}$ & Wall thinning & Erosion & Palisades & $11 / 1 / 2006$ \\
\hline 25 & Containment & CAC cooling coil / fitting & $\begin{array}{l}\text { CAC - Containment air } \\
\text { cooler }\end{array}$ & Wall thinning & Erosion & Palisades & $11 / 29 / 2006$ \\
\hline 215 & Containment & Liner & Containment & Degraded weld repair & NA & D.C. Cook 1 & $1 / 17 / 2000$ \\
\hline 213 & Containment & Liner & Containment & Pitting & Corrosion & D.C. Cook 1 & $3 / 5 / 1998$ \\
\hline 55 & Containment & $\begin{array}{l}\text { Small bore piping \& } \\
\text { tubing }\end{array}$ & $\begin{array}{l}\text { RCS - reactor coolant } \\
\text { system }\end{array}$ & Cracking & Vibration & Hope Creek 1 & $6 / 7 / 2005$ \\
\hline 56 & Containment & Test cap & Containment & Missing & Vibration & Oyster Creek & $7 / 12 / 2005$ \\
\hline 47 & Containment & Torus & Containment & Cracking & Fatigue & FitzPatrick & $6 / 27 / 2005$ \\
\hline 115 & Exchanger & CAC components & Containment & $\begin{array}{l}\text { Fouling, pitting, } \\
\text { rusting }\end{array}$ & Corrosion & Davis-Besse & $3 / 8 / 2002$ \\
\hline 48 & Exchanger & CAC cooling coil & Containment & Wall thinning & Erosion & Palisades & $10 / 9 / 2005$ \\
\hline 87 & Exchanger & $\begin{array}{l}\text { Condenser (including } \\
\text { ice) \& supports }\end{array}$ & $\begin{array}{l}\text { Condenser and condensate } \\
\text { system }\end{array}$ & Failure & Erosion & Duane Arnold & $11 / 25 / 2003$ \\
\hline 108 & Exchanger & Condensor / piping & $\begin{array}{l}\text { RCS - reactor coolant } \\
\text { system }\end{array}$ & Loss of material & Corrosion & Saint Lucie 2 & $4 / 1 / 2003$ \\
\hline 50 & Exchanger & $\begin{array}{l}\text { Condensor / Small bore } \\
\text { piping \& tubing }\end{array}$ & $\begin{array}{l}\text { RCS - reactor coolant } \\
\text { system }\end{array}$ & Cracking & Fatigue & Cooper & $9 / 23 / 2005$ \\
\hline
\end{tabular}




\begin{tabular}{|c|c|c|c|c|c|c|c|}
\hline ID & COMPONENT & SUBCOMPONENT & SYSTEM & AGING EFFECTS & AGING MECHANISMS & PLANT & $\begin{array}{l}\text { EVENT } \\
\text { DATE }\end{array}$ \\
\hline 113 & Exchanger & Condensor / tubing & $\begin{array}{l}\text { RCS - reactor coolant } \\
\text { system }\end{array}$ & Tear of plug & Under investigation & Palo Verde 1 & $3 / 27 / 2003$ \\
\hline 51 & Exchanger & Door & Containment & Failure & Corrosion & McGuire 1 & $9 / 17 / 2005$ \\
\hline 73 & Exchanger & Elbow & $\begin{array}{l}\text { CFCU - containment fan coil } \\
\text { unit }\end{array}$ & Wall thinning & Erosion & Prairie Island 2 & $11 / 17 / 2004$ \\
\hline 195 & Exchanger & ESW room cooler & $\begin{array}{l}\text { ESW - essential service } \\
\text { water }\end{array}$ & Fouling & $\begin{array}{l}\text { Macrofouling by Asiatic } \\
\text { clamshells }\end{array}$ & Callaway & $9 / 14 / 2000$ \\
\hline 24 & Exchanger & Exchanger & $\begin{array}{l}\text { CCW - component cooling } \\
\text { water }\end{array}$ & Failure & Unknown & Calvert Cliffs 1 & $1 / 17 / 2007$ \\
\hline 103 & Exchanger & Heat exchanger & CSS - containment spray & Fouling & Corrosion & Catawba 1 & $5 / 11 / 2003$ \\
\hline 93 & Exchanger & Heat exchanger & CSS - containment spray & Fouling & Corrosion & Catawba 1 & $10 / 9 / 2003$ \\
\hline 22 & Exchanger & Heat exchanger & EW - Essential cooling water & Fouling & Inorganic fouling & Palo Verde 2 & $12 / 22 / 2006$ \\
\hline 155 & Exchanger & Heat exchanger & SWS - service water system & Loss of material & NA & Crystal River 3 & $10 / 4 / 2001$ \\
\hline 45 & Exchanger & $\begin{array}{l}\text { Heat exchanger / Soft } \\
\text { iron gasket }\end{array}$ & $\begin{array}{l}\text { RHRSW - residual heat } \\
\text { removal service water }\end{array}$ & Loss of material & Corrosion & Browns Ferry 2 & $4 / 16 / 2005$ \\
\hline 179 & Exchanger & Inlet-sie tubesheet & $\begin{array}{l}\text { RCS - reactor coolant } \\
\text { system }\end{array}$ & Fouling & Corrosion at nearby pipe & D.C. Cook 1 & $2 / 15 / 2001$ \\
\hline 77 & Exchanger & Nozzle weld & $\begin{array}{l}\text { RCS - reactor coolant } \\
\text { system }\end{array}$ & Cracking & PWSCC & Catawba 2 & $9 / 16 / 2004$ \\
\hline 57 & Exchanger & Piping & $\begin{array}{l}\text { CCW - component cooling } \\
\text { water }\end{array}$ & Disintegration & Unknown & South Texas 2 & $7 / 11 / 2005$ \\
\hline 53 & Exchanger & Piping weld & $\begin{array}{l}\text { Condenser and condensate } \\
\text { system }\end{array}$ & Cracking & Fatigue & Turkey Point 4 & $12 / 25 / 2004$ \\
\hline 54 & Exchanger & Piping weld & $\begin{array}{l}\text { RCS - reactor coolant } \\
\text { system }\end{array}$ & Cracking & Fatigue & Palisades & $9 / 1 / 2005$ \\
\hline 63 & Exchanger & Piping weld & $\begin{array}{l}\text { RCS - reactor coolant } \\
\text { system }\end{array}$ & Cracking & PWSCC & Wolf Creek 1 & $4 / 15 / 2005$ \\
\hline 167 & Exchanger & Radiator cooling fin & $\begin{array}{l}\text { EDG - emergency diesel } \\
\text { generator }\end{array}$ & $\begin{array}{l}\text { Excessive } \\
\text { deformation }\end{array}$ & Corrosion & Saint Lucie 1 & $5 / 22 / 2001$ \\
\hline
\end{tabular}




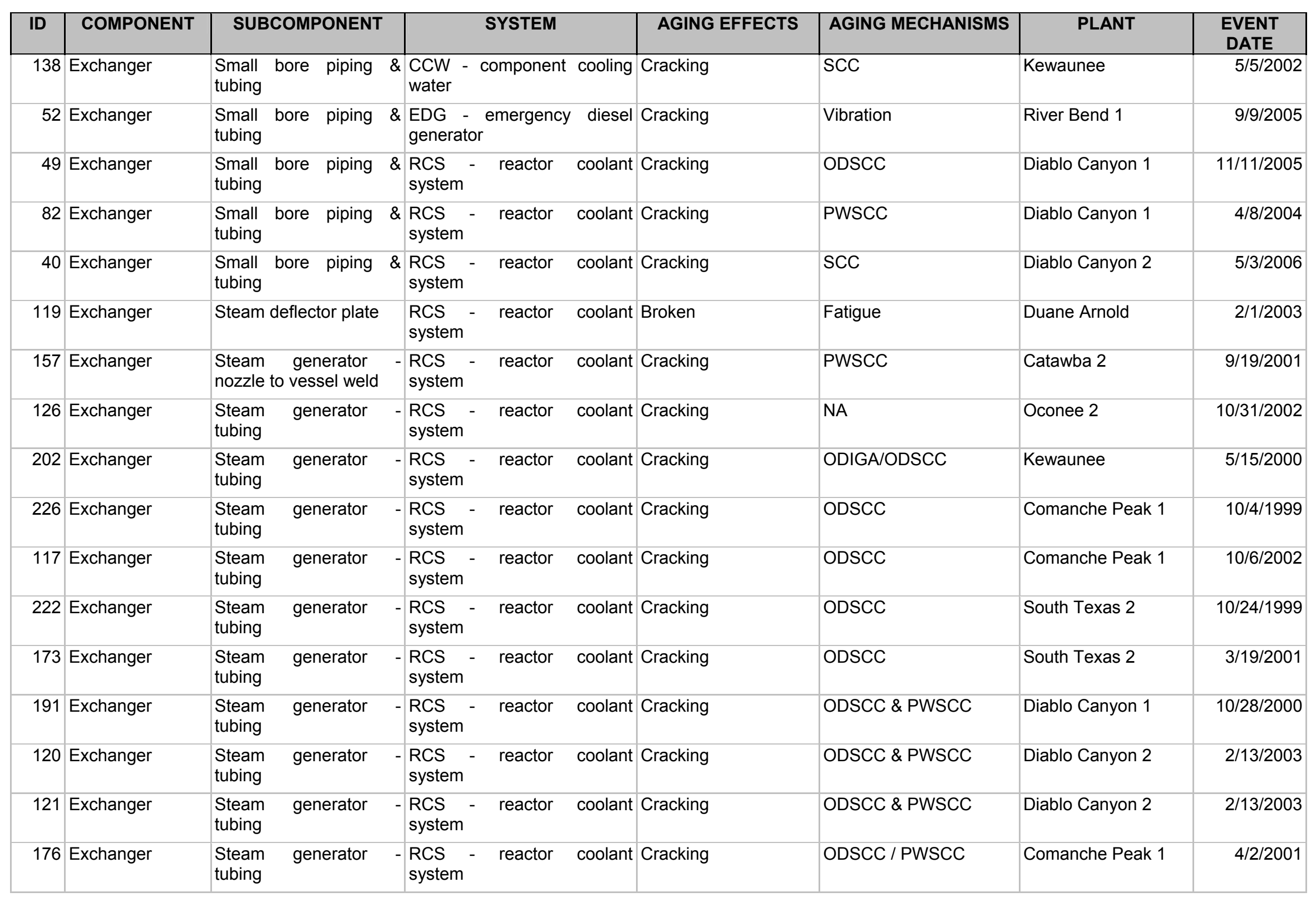




\begin{tabular}{|c|c|c|c|c|c|c|c|}
\hline ID & COMPONENT & SUBCOMPONENT & SYSTEM & AGING EFFECTS & AGING MECHANISMS & PLANT & $\begin{array}{l}\text { EVENT } \\
\text { DATE }\end{array}$ \\
\hline 217 & Exchanger & $\begin{array}{l}\text { Steam generator } \\
\text { tubing }\end{array}$ & $\begin{array}{l}\text { RCS - reactor coolant } \\
\text { system }\end{array}$ & Cracking & ODSCC / PWSCC & Farley 2 & $11 / 1 / 1999$ \\
\hline 185 & Exchanger & $\begin{array}{l}\text { Steam generator } \\
\text { tubing }\end{array}$ & $\begin{array}{l}\text { RCS - reactor coolant } \\
\text { system }\end{array}$ & Cracking & PWSCC & Indian Point 2 & $2 / 15 / 2000$ \\
\hline 205 & Exchanger & $\begin{array}{l}\text { Steam generator } \\
\text { tubing }\end{array}$ & $\begin{array}{l}\text { RCS - reactor coolant } \\
\text { system }\end{array}$ & Cracking & PWSCC & Indian Point 2 & $3 / 23 / 2000$ \\
\hline 180 & Exchanger & $\begin{array}{l}\text { Steam generator } \\
\text { tubing }\end{array}$ & $\begin{array}{l}\text { RCS - reactor coolant } \\
\text { system }\end{array}$ & Cracking & PWSCC & Indian Point 2 & $3 / 23 / 2000$ \\
\hline 142 & Exchanger & $\begin{array}{l}\text { Steam generator } \\
\text { tubing }\end{array}$ & $\begin{array}{l}\text { RCS - reactor coolant } \\
\text { system }\end{array}$ & $\begin{array}{l}\text { Cracking at tube } \\
\text { support plate }\end{array}$ & ODSCC & Diablo Canyon 1 & $5 / 19 / 2002$ \\
\hline 131 & Exchanger & $\begin{array}{l}\text { Steam generator } \\
\text { tubing }\end{array}$ & $\begin{array}{l}\text { RCS - reactor coolant } \\
\text { system }\end{array}$ & Defective & NA & Callaway & $11 / 5 / 2002$ \\
\hline 208 & Exchanger & $\begin{array}{l}\text { Steam generator } \\
\text { tubing }\end{array}$ & $\begin{array}{l}\text { RCS - reactor coolant } \\
\text { system }\end{array}$ & Degradation & IGA/SCC & Turkey Point 3 & $3 / 11 / 2000$ \\
\hline 210 & Exchanger & $\begin{array}{l}\text { Steam generator } \\
\text { tubing }\end{array}$ & $\begin{array}{l}\text { RCS - reactor coolant } \\
\text { system }\end{array}$ & Rupture & PWSCC & Indian Point 2 & $2 / 15 / 2000$ \\
\hline 8 & Exchanger & Tube plug & AR - Condenser air removal & Failure & $\begin{array}{l}\text { Corrosion / Galvanic } \\
\text { interaction }\end{array}$ & Palo Verde 2 & $10 / 6 / 2007$ \\
\hline 216 & Filter & Charcoal & $\begin{array}{l}\text { Control room makeup and } \\
\text { cleanup filtration }\end{array}$ & Degradation & Aging/end of life & South Texas 1 & $8 / 12 / 1999$ \\
\hline 190 & Filter & Charcoal & $\begin{array}{l}\text { ECF }-\quad \text { emergency } \\
\text { containment filter }\end{array}$ & Failure & Aging/end of life & Turkey Point 4 & $10 / 5 / 2000$ \\
\hline 223 & Filter & Charcoal & $\begin{array}{l}\text { SBGT - standby gas } \\
\text { treatment }\end{array}$ & Degradation & NA & FitzPatrick & 10/14/1999 \\
\hline 225 & Filter & Damper & CR - control room & Failure & $\begin{array}{l}\text { Gradual loosening of a } \\
\text { set screw }\end{array}$ & Three Mile Island 1 & 3/10/1999 \\
\hline 43 & Filter & Damper & Emergency exhaust system & Failure & Ice load & Callaway & $3 / 10 / 2006$ \\
\hline 214 & Filter & $\begin{array}{l}\text { Nuts holding in } \\
\text { Charcoal tray }\end{array}$ & $\begin{array}{l}\text { ECCS Pump room exhaust } \\
\text { filtration }\end{array}$ & Loosening & NA & Calvert Cliffs 1 & $1 / 28 / 2000$ \\
\hline 98 & Filter & Screens & $\begin{array}{l}\text { CW/ESW - Circulating water } \\
\text { and ESW }\end{array}$ & Failure & Corrosion & D.C. Cook 1 & $4 / 24 / 2003$ \\
\hline 165 & Filter & Screens & SWS - service water system & Failure & Organisms - fish & Point Beach 2 & $6 / 27 / 2001$ \\
\hline
\end{tabular}




\begin{tabular}{|c|c|c|c|c|c|c|c|}
\hline ID & COMPONENT & SUBCOMPONENT & SYSTEM & AGING EFFECTS & AGING MECHANISMS & PLANT & $\begin{array}{l}\text { EVENT } \\
\text { DATE }\end{array}$ \\
\hline 36 & Filter & Sealant (RTV) & $\begin{array}{l}\text { CREFS - Control room } \\
\text { emergency filtration }\end{array}$ & Failure & Aging/degraded & Point Beach 1 & $5 / 30 / 2006$ \\
\hline 161 & Filter & Strainer basket & $\begin{array}{l}\text { ESW - essential service } \\
\text { water }\end{array}$ & Failure & NA & D.C. Cook 1 & $8 / 29 / 2001$ \\
\hline 160 & Filter & Strainer basket & $\begin{array}{l}\text { ESW - essential service } \\
\text { water }\end{array}$ & Failure & NA & D.C. Cook 2 & $8 / 29 / 2001$ \\
\hline 106 & Filter & $\begin{array}{l}\text { TWS - traveling water } \\
\text { screen }\end{array}$ & $\begin{array}{l}\text { CW/ESW - Circulating water } \\
\text { and ESW }\end{array}$ & Failure & Corrosion & D.C. Cook 1 & $4 / 24 / 2003$ \\
\hline 164 & Other & Flood panel & NA & Deterioration & NA & Prairie Island 1 & $7 / 10 / 2001$ \\
\hline 114 & Other & $\begin{array}{l}\text { Gypsum } \\
\text { assembly }\end{array}$ & Fire barrier penetration & Surface cracking & $\begin{array}{l}\text { Aging/degraded/vibratio } \\
\mathrm{n}\end{array}$ & Monticello & $3 / 13 / 2003$ \\
\hline 125 & Other & Static line hanger & $345 \mathrm{KV}$ transmission line & Failure & Mechanical wear & Palisades & $12 / 1 / 2002$ \\
\hline 34 & Piping system & Cooling coil & SWS - service water system & Cracking & Erosion & Duane Arnold & $6 / 30 / 2006$ \\
\hline 163 & Piping system & Cooling coil & SWS - service water system & Loss of material & Flow induced erosion & Vermont Yankee & $9 / 25 / 2001$ \\
\hline 158 & Piping system & Drain trap outlet plug & $\begin{array}{l}\mathrm{HPCl} \text { - high pressure coolant } \\
\text { injection }\end{array}$ & Failure & $\begin{array}{l}\text { FAC - Flow Accelerated } \\
\text { Corrosion }\end{array}$ & Duane Arnold & $9 / 2 / 2001$ \\
\hline 30 & Piping system & Elbow & $\mathrm{HPCl} / \mathrm{RCIC}$ & Cracking & Erosion & Peach Bottom 2 & $10 / 7 / 2006$ \\
\hline 211 & Piping system & Elbow & $\begin{array}{l}\text { RCS - reactor coolant } \\
\text { system }\end{array}$ & Cracking & Thermal fatigue & Oconee 1 & $2 / 16 / 2000$ \\
\hline 33 & Piping system & Fitting & $\begin{array}{l}\text { EDG - emergency diesel } \\
\text { generator }\end{array}$ & Cracking & Fatigue & Kewaunee & $8 / 17 / 2006$ \\
\hline 14 & Piping system & Fitting & $\begin{array}{l}\text { EHC - electro hydraulic } \\
\text { control system }\end{array}$ & Cracking & Mechanical loads & Browns Ferry 1 & $5 / 24 / 2007$ \\
\hline 197 & Piping system & Fitting & $\begin{array}{l}\text { EHC - electro hydraulic } \\
\text { control system }\end{array}$ & Leak & NA & FitzPatrick & $8 / 27 / 2000$ \\
\hline 26 & Piping system & Fitting & $\begin{array}{l}\text { HVAC - Heating, ventilation } \\
\text { and air conditioning }\end{array}$ & Loosening & Vibration & Dresden 2 & $11 / 8 / 2006$ \\
\hline 86 & Piping system & Fitting & $\begin{array}{l}\text { RCS - reactor coolant } \\
\text { system }\end{array}$ & Cracking & Fatigue & Oconee 1 & $1 / 8 / 2004$ \\
\hline
\end{tabular}




\begin{tabular}{|c|c|c|c|c|c|c|c|}
\hline ID & COMPONENT & SUBCOMPONENT & SYSTEM & AGING EFFECTS & AGING MECHANISMS & PLANT & $\begin{array}{l}\text { EVENT } \\
\text { DATE }\end{array}$ \\
\hline 193 & Piping system & Fitting & RR - reactor recirculation & Cracking & Fatigue & Dresden 3 & $3 / 21 / 1999$ \\
\hline 13 & Piping system & Hose & $\begin{array}{l}\text { RCS - reactor coolant } \\
\text { system }\end{array}$ & Cracking & IGSCC & Clinton & $6 / 18 / 2007$ \\
\hline 78 & Piping system & Nozzle & $\begin{array}{l}\text { CCW - component cooling } \\
\text { water }\end{array}$ & Cracking & Fatigue & Diablo Canyon 1 & $7 / 21 / 2004$ \\
\hline 70 & Piping system & Nozzle & $\begin{array}{l}\text { RCS - reactor coolant } \\
\text { system }\end{array}$ & Cracking & PWSCC & Calvert Cliffs 2 & $2 / 26 / 2005$ \\
\hline 224 & Piping system & Nozzle & $\begin{array}{l}\text { RCS - reactor coolant } \\
\text { system }\end{array}$ & Cracking & PWSCC & Palo Verde 1 & $10 / 2 / 1999$ \\
\hline 172 & Piping system & Nozzle & $\begin{array}{l}\text { RCS - reactor coolant } \\
\text { system }\end{array}$ & Cracking & PWSCC & Palo Verde 1 & $3 / 31 / 2001$ \\
\hline 109 & Piping system & Nozzle & $\begin{array}{l}\text { RCS - reactor coolant } \\
\text { system }\end{array}$ & Cracking & PWSCC & Palo Verde 3 & $3 / 29 / 2003$ \\
\hline 169 & Piping system & Nozzle & $\begin{array}{l}\text { RCS - reactor coolant } \\
\text { system }\end{array}$ & Cracking & PWSCC & Saint Lucie 1 & $4 / 14 / 2001$ \\
\hline 91 & Piping system & Nozzle & $\begin{array}{l}\text { RCS - reactor coolant } \\
\text { system }\end{array}$ & Cracking & PWSCC & Waterford 3 & $10 / 24 / 2003$ \\
\hline 5 & Piping system & $\begin{array}{l}\text { nozzle-to-elbow } \\
\text { dissimilar metal butt } \\
\text { weld }\end{array}$ & $\begin{array}{l}\text { RCS - reactor coolant } \\
\text { system }\end{array}$ & Loss of material & PWSCC & Davis-Besse & $1 / 4 / 2008$ \\
\hline 39 & Piping system & Nozzle-to-elbow weld & $\begin{array}{l}\text { RCS - reactor coolant } \\
\text { system }\end{array}$ & Cracking & PWSCC & Davis-Besse & $3 / 18 / 2006$ \\
\hline 181 & Piping system & Nozzle-to-pipe weld & $\begin{array}{l}\text { RCS - reactor coolant } \\
\text { system }\end{array}$ & Cracking & PWSCC & Summer & $10 / 12 / 2000$ \\
\hline 149 & Piping system & Pipe-to-elbow weld & SWS - service water system & Loss of material & Erosion & Indian Point 2 & $10 / 29 / 2001$ \\
\hline 20 & Piping system & Piping & $\begin{array}{l}\text { Auxiliary steam piping } \\
\text { system }\end{array}$ & Plugging & Corrosion & Quad Cities 2 & $2 / 28 / 2007$ \\
\hline 32 & Piping system & Piping & $\begin{array}{l}\text { Condenser and condensate } \\
\text { system }\end{array}$ & Broken & Fatigue & Davis-Besse & $9 / 6 / 2006$ \\
\hline 177 & Piping system & Piping & $\begin{array}{l}\text { EDG - emergency diesel } \\
\text { generator }\end{array}$ & Cracking & Fatigue & Grand Gulf 1 & $2 / 19 / 2001$ \\
\hline 67 & Piping system & Piping & $\begin{array}{l}\text { ESW - essential service } \\
\text { water }\end{array}$ & Wall thinning & $\begin{array}{l}\text { Under-deposit corrosion } \\
\text { and Microbiologically }\end{array}$ & Callaway & $3 / 26 / 2005$ \\
\hline
\end{tabular}




\begin{tabular}{|c|c|c|c|c|c|c|c|}
\hline ID & COMPONENT & SUBCOMPONENT & SYSTEM & AGING EFFECTS & AGING MECHANISMS & PLANT & $\begin{array}{l}\text { EVENT } \\
\text { DATE }\end{array}$ \\
\hline & & & & & $\begin{array}{l}\text { influenced corrosion } \\
\text { (MIC) }\end{array}$ & & \\
\hline 107 & Piping system & Piping & $\begin{array}{l}\mathrm{HPCl} \text { - high pressure coolant } \\
\text { injection }\end{array}$ & Cracking & Mechanical loads & Duane Arnold & $4 / 20 / 2003$ \\
\hline 7 & Piping system & Piping & $\begin{array}{l}\mathrm{HPCl} \text { - high pressure coolant } \\
\text { injection }\end{array}$ & Leaking & Corrosion & Browns Ferry 3 & $11 / 30 / 2007$ \\
\hline 19 & Piping system & Piping & $\begin{array}{l}\mathrm{HPCl} \text { - high pressure coolant } \\
\text { injection }\end{array}$ & Wall thinning & $\begin{array}{l}\text { FAC - Flow Accelerated } \\
\text { Corrosion }\end{array}$ & Dresden 3 & $3 / 2 / 2007$ \\
\hline 187 & Piping system & Piping & $\begin{array}{l}\text { Main generator hydrogen } \\
\text { cooling }\end{array}$ & Cracking & Fatigue & Arkansas Nuclear 1 & $1 / 5 / 2001$ \\
\hline 204 & Piping system & Piping & Moisture separator reheater & Rupture & $\begin{array}{l}\text { FAC - Flow Accelerated } \\
\text { Corrosion }\end{array}$ & Callaway & 8/11/1999 \\
\hline 194 & Piping system & Piping & $\begin{array}{l}\text { RCS - reactor coolant } \\
\text { system }\end{array}$ & Broken & Fatigue & $\begin{array}{l}\text { Columbia Generating } \\
\text { Station }\end{array}$ & $9 / 18 / 2000$ \\
\hline 139 & Piping system & Piping & RHR - residual heat removal & Cracking & $\begin{array}{l}\text { TGSCC - chemical } \\
\text { attack }\end{array}$ & Surry 2 & $4 / 13 / 2002$ \\
\hline 116 & Piping system & Piping & SWS - service water system & $\begin{array}{l}\text { Fouling, pitting, } \\
\text { rusting }\end{array}$ & Corrosion & Davis-Besse & $3 / 8 / 2002$ \\
\hline 2 & Piping system & Piping & UHS - ultimate heat sink & Wall thinning/Failure & $\begin{array}{l}\text { General and pitting } \\
\text { corrosion }\end{array}$ & Byron 1 & $10 / 19 / 2007$ \\
\hline 3 & Piping system & Piping & UHS - ultimate heat sink & Wall thinning/Failure & $\begin{array}{l}\text { General and pitting } \\
\text { corrosion }\end{array}$ & Byron 2 & $10 / 19 / 2007$ \\
\hline 200 & Piping system & Piping weld & $\begin{array}{l}\text { CCSW - containment cooling } \\
\text { service water }\end{array}$ & Leak & NA & Dresden 2 & $6 / 28 / 2000$ \\
\hline 133 & Piping system & Piping weld & $\begin{array}{l}\text { ECCS - emergency core } \\
\text { cooling system }\end{array}$ & Cracking & Fatigue & Millstone 2 & $8 / 3 / 2002$ \\
\hline 198 & Piping system & Piping weld & $\begin{array}{l}\text { EHC - electro hydraulic } \\
\text { control system }\end{array}$ & Leak & NA & FitzPatrick & $8 / 27 / 2000$ \\
\hline 129 & Piping system & Piping weld & HPI - high pressure injection & Cracking & $\begin{array}{l}\text { Fatigue } \quad w / \quad \text { initiating } \\
\text { defects }\end{array}$ & Arkansas Nuclear 1 & $10 / 5 / 2002$ \\
\hline 134 & Piping system & Piping weld & RCP - reactor coolant pump & Cracking & Fatigue & Calvert Cliffs 1 & $7 / 24 / 2002$ \\
\hline 75 & Piping system & Piping weld & $\begin{array}{l}\text { RCS - reactor coolant } \\
\text { system }\end{array}$ & Cracking & Fatigue & Hope Creek 1 & $10 / 10 / 2004$ \\
\hline
\end{tabular}




\begin{tabular}{|c|c|c|c|c|c|c|c|}
\hline ID & COMPONENT & SUBCOMPONENT & SYSTEM & AGING EFFECTS & AGING MECHANISMS & PLANT & $\begin{array}{l}\text { EVENT } \\
\text { DATE }\end{array}$ \\
\hline 85 & Piping system & Piping weld & $\begin{array}{l}\text { RCS - reactor coolant } \\
\text { system }\end{array}$ & Cracking & Fatigue & Palo Verde 1 & $2 / 3 / 2004$ \\
\hline 71 & Piping system & Piping weld & $\begin{array}{l}\text { RCS - reactor coolant } \\
\text { system }\end{array}$ & Cracking & Fatigue & Saint Lucie 2 & $2 / 10 / 2005$ \\
\hline 72 & Piping system & Piping weld & $\begin{array}{l}\text { RCS - reactor coolant } \\
\text { system }\end{array}$ & Cracking & Fatigue & South Texas 2 & $2 / 9 / 2005$ \\
\hline 81 & Piping system & Piping weld & $\begin{array}{l}\text { RCS - reactor coolant } \\
\text { system }\end{array}$ & Cracking & Fatigue & Summer & $3 / 30 / 2004$ \\
\hline 44 & Piping system & Piping weld & $\begin{array}{l}\text { RCS - reactor coolant } \\
\text { system }\end{array}$ & Cracking & Fatigue & Vogtle 2 & $2 / 1 / 2006$ \\
\hline 88 & Piping system & Piping weld & $\begin{array}{l}\text { RCS - reactor coolant } \\
\text { system }\end{array}$ & Cracking & Fatigue & Wolf Creek 1 & $11 / 17 / 2003$ \\
\hline 58 & Piping system & Piping weld & $\begin{array}{l}\text { RHR SDC- RHR shutdown } \\
\text { cooling }\end{array}$ & Cracking & Fatigue & FitzPatrick & $7 / 4 / 2005$ \\
\hline 127 & Piping system & Piping weld & RR - reactor recirculation & Cracking & Fatigue & Dresden 3 & $10 / 8 / 2002$ \\
\hline 152 & Piping system & Piping weld & RR - reactor recirculation & Cracking & Fatigue & Hope Creek 1 & $10 / 10 / 2001$ \\
\hline 66 & Piping system & Piping weld & RS - Recirculation system & Cracking & Fatigue & Hope Creek 1 & $3 / 27 / 2005$ \\
\hline 68 & Piping system & Piping weld & RS - Recirculation system & Cracking & Fatigue & Susquehanna 2 & $3 / 20 / 2005$ \\
\hline 83 & Piping system & Piping weld & RS - Recirculation system & Cracking & IGSCC & Quad Cities 2 & $3 / 9 / 2004$ \\
\hline 123 & Piping system & Piping weld & RS - Recirculation system & Cracking & Vibration & Dresden 3 & $12 / 7 / 2002$ \\
\hline 46 & Piping system & Piping weld & $\begin{array}{l}\text { SDCS - Shutdown cooling } \\
\text { system }\end{array}$ & Cracking & Fatigue & San Onofre 2 & $1 / 11 / 2006$ \\
\hline 220 & Piping system & Piping weld & $\begin{array}{l}\text { Steam Generator Channel } \\
\text { Head Drain Isolation }\end{array}$ & Cracking & NA & Point Beach 1 & $11 / 4 / 1999$ \\
\hline 137 & Piping system & Piping weld & SWS - service water system & Wall thinning & Corrosion & Indian Point 3 & $6 / 7 / 2002$ \\
\hline 11 & Piping system & Piping/Elbow & $\begin{array}{l}\mathrm{HPCl} \text { - high pressure coolant } \\
\text { injection }\end{array}$ & Wall thinning/leaking & Erosion & Dresden 2 & $7 / 26 / 2007$ \\
\hline
\end{tabular}




\begin{tabular}{|c|c|c|c|c|c|c|c|}
\hline ID & COMPONENT & SUBCOMPONENT & SYSTEM & AGING EFFECTS & AGING MECHANISMS & PLANT & $\begin{array}{l}\text { EVENT } \\
\text { DATE }\end{array}$ \\
\hline 6 & Piping system & $\begin{array}{l}\text { Reactor coolant pump } \\
\text { seal weld }\end{array}$ & $\begin{array}{lll}\text { RCS - reactor coolant } \\
\text { system }\end{array}$ & Cracking/leaking & Fatigue & Saint Lucie 2 & $12 / 21 / 2007$ \\
\hline 150 & Piping system & $\begin{array}{l}\text { Small bore piping \& } \\
\text { tubing }\end{array}$ & Containment & Broken & Mechanical loads & Vermont Yankee & $10 / 25 / 2001$ \\
\hline 207 & Piping system & $\begin{array}{l}\text { Small bore piping \& } \\
\text { tubing }\end{array}$ & $\begin{array}{l}\text { Control room air conditioning } \\
\text { chiller }\end{array}$ & Leak & Wear/deterioration & Oconee 1 & $3 / 9 / 2000$ \\
\hline 201 & Piping system & $\begin{array}{l}\text { Small bore piping \& } \\
\text { tubing }\end{array}$ & $\begin{array}{l}\text { EHC - electro hydraulic } \\
\text { control system }\end{array}$ & Cracking & Corrosion fatigue & Robinson 2 & $6 / 21 / 2000$ \\
\hline 104 & Piping system & $\begin{array}{l}\text { Small bore piping \& } \\
\text { tubing }\end{array}$ & $\begin{array}{l}\text { EHC - electro hydraulic } \\
\text { control system }\end{array}$ & Cracking & Fatigue & River Bend 1 & $2 / 22 / 2003$ \\
\hline 9 & Piping system & $\begin{array}{l}\text { Small bore piping \& } \\
\text { tubing }\end{array}$ & $\begin{array}{l}\text { EHC - electro hydraulic } \\
\text { control system }\end{array}$ & Cracking/leaking & Mechanical wear & Browns Ferry 1 & 9/3/2007 \\
\hline 206 & Piping system & $\begin{array}{l}\text { Small bore piping \& } \\
\text { tubing }\end{array}$ & $\begin{array}{l}\text { Electric board room air } \\
\text { conditioning unit }\end{array}$ & Broken & Aging \& fatigue & Watts Bar 1 & $1 / 2 / 1999$ \\
\hline 219 & Piping system & $\begin{array}{l}\text { Small bore piping \& } \\
\text { tubing }\end{array}$ & $\begin{array}{l}\text { Extraction Steam System } \\
\text { Moisture } \\
\text { Reheater Drain Tank }\end{array}$ & Failure & Wear & FitzPatrick & $11 / 5 / 1999$ \\
\hline 111 & Piping system & $\begin{array}{l}\text { Small bore piping \& } \\
\text { tubing }\end{array}$ & $\begin{array}{l}\text { PCIS - primary containment } \\
\text { isolation system }\end{array}$ & Cracking & Fatigue & Peach Bottom 2 & $4 / 12 / 2003$ \\
\hline 65 & Piping system & $\begin{array}{l}\text { Small bore piping \& } \\
\text { tubing }\end{array}$ & $\begin{array}{l}\text { RCS - reactor coolant } \\
\text { system }\end{array}$ & Loss of material & TGSCC & Salem 2 & $4 / 4 / 2005$ \\
\hline 146 & Piping system & $\begin{array}{l}\text { Small bore piping \& } \\
\text { tubing }\end{array}$ & $\begin{array}{l}\text { RSS - recirculation spray } \\
\text { system }\end{array}$ & Fouling & Corrosion & Beaver Valley 2 & $1 / 11 / 2002$ \\
\hline 12 & Piping system & Snubber & $\begin{array}{l}\text { EDG - emergency diesel } \\
\text { generator }\end{array}$ & Cracking & $\begin{array}{l}\text { Temperature - heat } \\
\text { treatment }\end{array}$ & Palisades & $2 / 22 / 2007$ \\
\hline 18 & Piping system & Socket weld & $\begin{array}{l}\text { RCS - reactor coolant } \\
\text { system }\end{array}$ & Cracking & Fatigue & Hatch 2 & $3 / 9 / 2007$ \\
\hline 10 & Piping system & Socket weld fitting & $\begin{array}{l}\text { RCS - reactor coolant } \\
\text { system }\end{array}$ & Cracking/leaking & Fatigue (high cycle) & Saint Lucie 2 & $8 / 18 / 2007$ \\
\hline 209 & Piping system & Suction line weld & Charging pump & Cracking & NA & Waterford 3 & $3 / 6 / 2000$ \\
\hline 122 & Piping system & Support & $\begin{array}{l}\mathrm{HPCl} \text { - high pressure coolant } \\
\text { injection }\end{array}$ & Failure & $\begin{array}{l}\text { Hydrodynamic transient } \\
\text { / water hammer }\end{array}$ & Dresden 3 & $7 / 5 / 2001$ \\
\hline 186 & Piping system & Support & $\begin{array}{l}\text { Main generator hydrogen } \\
\text { cooling }\end{array}$ & Failure & Vibration & Arkansas Nuclear 1 & $1 / 5 / 2001$ \\
\hline
\end{tabular}




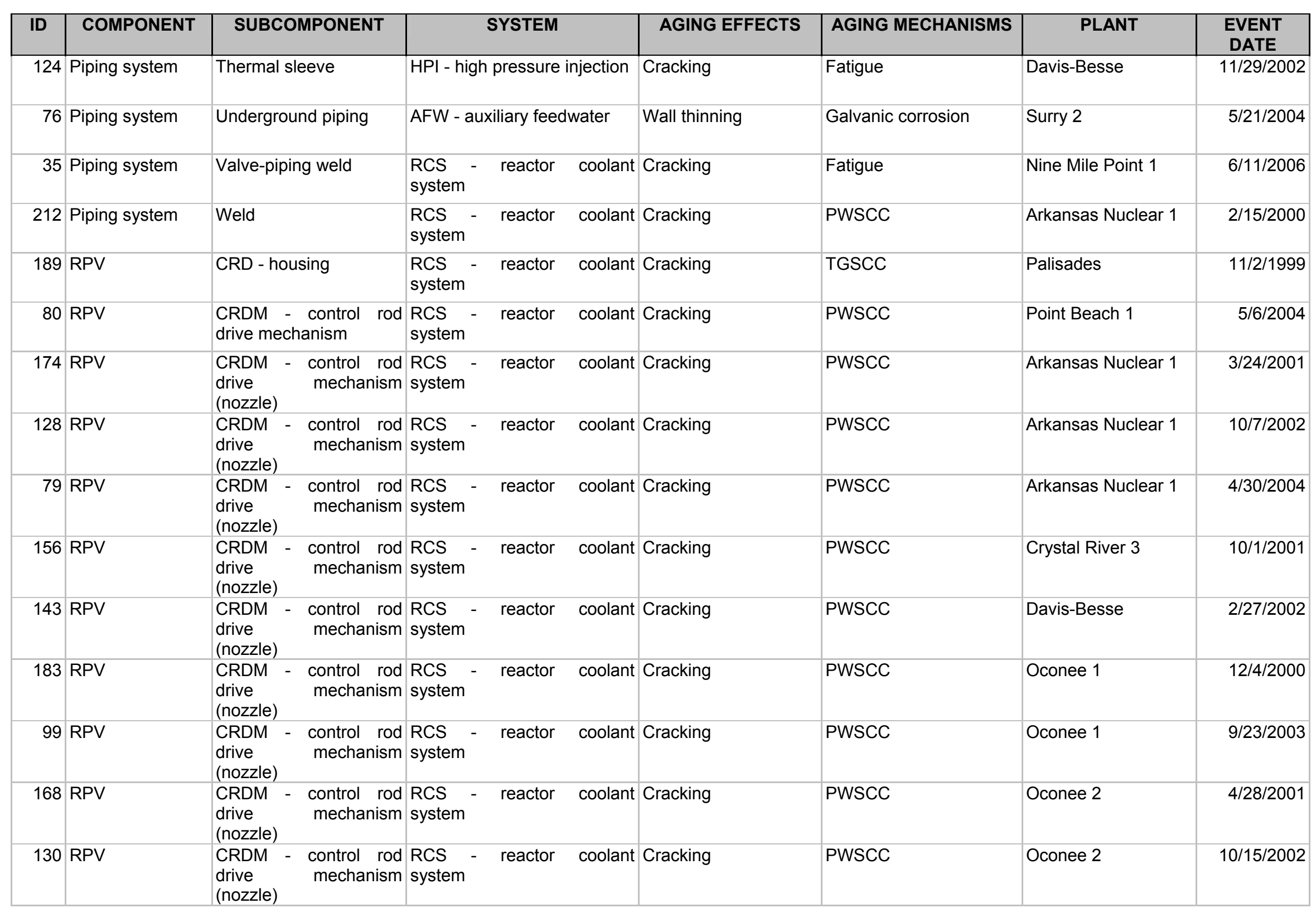




\begin{tabular}{|c|c|c|c|c|c|c|c|c|c|}
\hline ID & COMPONENT & SUBCOMPONENT & \multicolumn{3}{|c|}{ SYSTEM } & AGING EFFECTS & AGING MECHANISMS & PLANT & EVENT \\
\hline 59 & RPV & $\begin{array}{ll}\text { CRDM } & \text { - control rod } \\
\text { drive } & \text { mechanism } \\
\text { (nozzle) } & \end{array}$ & $\begin{array}{l}\text { RCS } \\
\text { system }\end{array}$ & - reactor & coolant & Cracking & PWSCC & Oconee 3 & $2 / 18 / 2001$ \\
\hline 175 & RPV & $\begin{array}{l}\text { CRDM - } \begin{array}{l}\text { control rod } \\
\text { drive } \\
\text { (nozzle) }\end{array} \\
\text { mechanism }\end{array}$ & $\begin{array}{l}\text { RCS } \\
\text { system }\end{array}$ & - reactor & coolant & Cracking & PWSCC & Oconee 3 & $2 / 18 / 2001$ \\
\hline 148 & RPV & $\begin{array}{l}\text { CRDM - control rod } \\
\text { drive } \\
\text { (nozzle) }\end{array}$ & $\begin{array}{l}\text { RCS } \\
\text { system }\end{array}$ & - reactor & coolant & Cracking & PWSCC & Oconee 3 & $11 / 12 / 2001$ \\
\hline 112 & RPV & $\begin{array}{l}\text { CRDM - control rod } \\
\text { drive } \\
\text { (nozzle) }\end{array}$ & $\begin{array}{l}\text { RCS } \\
\text { system }\end{array}$ & - reactor & coolant & Cracking & PWSCC & Oconee 3 & $5 / 2 / 2003$ \\
\hline 74 & RPV & $\begin{array}{l}\text { CRDM - } \\
\begin{array}{l}\text { control rod } \\
\text { (nozzle) }\end{array}\end{array}$ & $\begin{array}{l}\text { RCS } \\
\text { system }\end{array}$ & - reactor & coolant & Cracking & PWSCC & Palisades & $10 / 16 / 2004$ \\
\hline 153 & RPV & $\begin{array}{l}\text { CRDM - control rod } \\
\text { drive } \\
\text { (nozzle) }\end{array}$ & $\begin{array}{l}\text { RCS } \\
\text { system }\end{array}$ & - reactor & coolant & Cracking & PWSCC & Three Mile Island 1 & $10 / 12 / 2001$ \\
\hline 145 & RPV & CRDM - upper housing & $\begin{array}{l}\text { RCS } \\
\text { system }\end{array}$ & - reactor & coolant & Cracking & TGSCC & Palisades & $6 / 21 / 2001$ \\
\hline 151 & RPV & CRDM penetration & $\begin{array}{l}\text { RCS } \\
\text { system }\end{array}$ & - reactor & coolant & Cracking & PWSCC & Surry 1 & $10 / 28 / 2001$ \\
\hline 170 & RPV & CRDM Seal housing & $\begin{array}{l}\text { PCS } \\
\text { system }\end{array}$ & - primary & coolant & Cracking & TGSCC & Palisades & $3 / 31 / 2001$ \\
\hline 221 & RPV & CRDM Seal housing & $\begin{array}{l}\text { RCS } \\
\text { system }\end{array}$ & - reactor & coolant & Cracking & TGSCC & Palisades & 10/16/1999 \\
\hline 162 & RPV & Feedwater nozzle & $\begin{array}{l}\text { RCS } \\
\text { system }\end{array}$ & - reactor & coolant & Fouling & Inorganic fouling & Vermont Yankee & $8 / 21 / 2001$ \\
\hline 16 & RPV & Nozzle & $\begin{array}{l}\text { RCS } \\
\text { system }\end{array}$ & - reactor & coolant & Cracking & PWSCC & Byron 2 & $4 / 9 / 2007$ \\
\hline 141 & RPV & Nozzle & $\begin{array}{l}\text { RCS } \\
\text { system }\end{array}$ & - reactor & coolant & Cracking & PWSCC & Oconee 1 & $4 / 1 / 2002$ \\
\hline 105 & RPV & Nozzle & $\begin{array}{l}\text { RCS } \\
\text { system }\end{array}$ & - reactor & coolant & Cracking & PWSCC & Saint Lucie 2 & $4 / 30 / 2003$ \\
\hline 100 & RPV & Nozzle & $\begin{array}{l}\text { RCS } \\
\text { system }\end{array}$ & - reactor & coolant & Cracking & SCC & South Texas 1 & $4 / 12 / 2003$ \\
\hline 21 & RPV & Nozzle weld & $\begin{array}{l}\text { RCS } \\
\text { system }\end{array}$ & - reactor & coolant & Cracking & IGSCC & Duane Arnold & $2 / 18 / 2007$ \\
\hline
\end{tabular}




\begin{tabular}{|c|c|c|c|c|c|c|c|}
\hline ID & COMPONENT & SUBCOMPONENT & SYSTEM & AGING EFFECTS & AGING MECHANISMS & PLANT & $\begin{array}{l}\text { EVENT } \\
\text { DATE }\end{array}$ \\
\hline 218 & RPV & Nozzle-safe end weld & RR - reactor recirculation & Cracking & IGSCC & Duane Arnold & $11 / 5 / 1999$ \\
\hline 95 & RPV & Nozzle-to-cap weld & $\begin{array}{l}\mathrm{RCS} \\
\text { system }\end{array}$ & Cracking & $\begin{array}{l}\text { IDSCC - Interdendritic } \\
\text { SCC }\end{array}$ & Pilgrim 1 & $10 / 1 / 2003$ \\
\hline 118 & RPV & Penetration & $\begin{array}{l}\text { RCS - reactor coolant } \\
\text { system }\end{array}$ & Cracking & PWSCC & North Anna 1 & $3 / 4 / 2003$ \\
\hline 132 & RPV & Penetration & $\begin{array}{l}\text { RCS - reactor coolant } \\
\text { system }\end{array}$ & Cracking & $\begin{array}{l}\text { PWSCC with initial hot- } \\
\text { short cracking }\end{array}$ & North Anna 2 & $9 / 14 / 2002$ \\
\hline 60 & RPV & Piping & $\begin{array}{l}\mathrm{RCS} \\
\text { system }\end{array}$ & Cracking & PWSCC & Palo Verde 2 & $4 / 23 / 2005$ \\
\hline 31 & RPV & Piping & $\begin{array}{l}\text { RCS - reactor coolant } \\
\text { system }\end{array}$ & Cracking & PWSCC & Palo Verde 2 & $10 / 7 / 2006$ \\
\hline 41 & RPV & Piping weld & CS - core spray & Cracking & IGSCC & Brunswick 1 & $3 / 21 / 2006$ \\
\hline 42 & RPV & Sleeve & HPI - high pressure injection & Cracking & Fatigue & Davis-Besse & $11 / 29 / 2002$ \\
\hline 101 & RPV & Steam dryer & $\begin{array}{l}\mathrm{RCS} \\
\text { system }\end{array}$ & Cracking & Fatigue & Quad Cities 2 & $6 / 12 / 2003$ \\
\hline 135 & RPV & Steam dryer & $\begin{array}{l}\text { RCS - reactor coolant } \\
\text { system }\end{array}$ & Failure & Fatigue & Quad Cities 2 & $7 / 11 / 2002$ \\
\hline 154 & RPV & $\begin{array}{l}\text { Thermocouple } \quad(T / C) \\
\text { nozzles }\end{array}$ & $\begin{array}{l}\text { RCS - reactor coolant } \\
\text { system }\end{array}$ & Cracking & PWSCC & Three Mile Island 1 & $10 / 12 / 2001$ \\
\hline 102 & RPV & Vent line & $\begin{array}{l}\text { RCS - reactor coolant } \\
\text { system }\end{array}$ & Cracking & Corrosion and fatigue & Quad Cities 1 & $5 / 20 / 2003$ \\
\hline 171 & Structural steel & Bolt & EFW - emergency feedwater & Loosening & Vibration & Three Mile Island 1 & $2 / 1 / 2001$ \\
\hline 159 & Structural steel & Door & Containment & Failure & NA & D.C. Cook 2 & $1 / 23 / 2001$ \\
\hline 184 & Structural steel & Door & Fire protecton & Failure & $\begin{array}{l}\text { Corrosion and dirt } \\
\text { buildup }\end{array}$ & Kewaunee & $1 / 18 / 2001$ \\
\hline 166 & Structural steel & Door & $\begin{array}{l}\text { Turbine Driven Auxiliary } \\
\text { Feedwater Pump enclosuer } \\
\text { door }\end{array}$ & $\begin{array}{l}\text { Degraded closure } \\
\text { mechanism }\end{array}$ & NA & Millstone 2 & $6 / 12 / 2001$ \\
\hline 182 & Structural steel & Pump hold-down beam & RS - Recirculation system & Failure & IGSCC & Quad Cities 1 & $1 / 9 / 2002$ \\
\hline
\end{tabular}




\begin{tabular}{|c|c|c|c|c|c|c|c|}
\hline ID & COMPONENT & SUBCOMPONENT & SYSTEM & AGING EFFECTS & AGING MECHANISMS & PLANT & $\begin{array}{l}\text { EVENT } \\
\text { DATE }\end{array}$ \\
\hline 178 & Structural steel & Screw & $\begin{array}{l}\text { RCS - reactor coolant } \\
\text { system }\end{array}$ & Failure & PWSCC & Summer & 4/13/1999 \\
\hline 196 & Structural steel & Weld & $\begin{array}{l}\text { CREACUS - control room } \\
\text { emergency cleanup }\end{array}$ & Cracking & NA & San Onofre 2 & $9 / 1 / 2000$ \\
\hline 17 & Tank & $\begin{array}{l}\text { Filter tanks and internal } \\
\text { components }\end{array}$ & SWS - service water system & Cracking & Corrosion & Kewaunee & $5 / 30 / 2006$ \\
\hline 147 & Tank & Floating cover & $\begin{array}{l}\text { CST - condensate storage } \\
\text { tank }\end{array}$ & Deterioration & $\begin{array}{l}\text { Wear accelerated by } \\
\text { Nitrogen }\end{array}$ & Callaway & $12 / 3 / 2001$ \\
\hline 61 & Tank & Piping weld & $\begin{array}{l}\text { ECCS - emergency core } \\
\text { cooling system }\end{array}$ & Cracking & SCC - Chemical attack & Salem 1 & $4 / 19 / 2005$ \\
\hline 28 & Tank & SLC tank & SLC - standby liquid control & Cracking & SCC - Chemical attack & Quad Cities 1 & $10 / 12 / 2006$ \\
\hline 1 & Tank & $\begin{array}{l}\text { Tank shell - sensor } \\
\text { connection }\end{array}$ & SLC - standby liquid control & Cracking & TGSCC & Dresden 2 & $1 / 18 / 2007$ \\
\hline 15 & Vessel & Nozzle weld & $\begin{array}{l}\text { RCS - reactor coolant } \\
\text { system }\end{array}$ & Cracking & $\begin{array}{l}\text { IDSCC - Interdendritic } \\
\text { SCC }\end{array}$ & Pilgrim 1 & $4 / 26 / 2007$ \\
\hline 29 & Vessel & Nozzle-safe end weld & $\begin{array}{l}\text { RCS - reactor coolant } \\
\text { system }\end{array}$ & Cracking & PWSCC & Wolf Creek 1 & $10 / 11 / 2006$ \\
\hline 90 & Vessel & Pressurizer & $\begin{array}{l}\text { RCS - reactor coolant } \\
\text { system }\end{array}$ & Cracking & PWSCC & Three Mile Island 1 & $11 / 4 / 2003$ \\
\hline 97 & Vessel & $\begin{array}{l}\text { Pressurizer - filters and } \\
\text { screens }\end{array}$ & $\begin{array}{l}\text { RCS - reactor coolant } \\
\text { system }\end{array}$ & Failure & Corrosion & Davis-Besse & $4 / 10 / 1998$ \\
\hline 144 & Vessel & $\begin{array}{l}\text { Pressurizer - } \quad \text { heater } \\
\text { sleeve penetrations }\end{array}$ & $\begin{array}{l}\text { RCS - reactor coolant } \\
\text { system }\end{array}$ & Cracking & PWSCC & Millstone 2 & $2 / 19 / 2002$ \\
\hline 199 & Vessel & $\begin{array}{l}\text { Pressurizer heater } \\
\text { sleeves }\end{array}$ & $\begin{array}{l}\text { RCS - reactor coolant } \\
\text { system }\end{array}$ & Cracking & PWSCC & Arkansas Nuclear 2 & $7 / 30 / 2000$ \\
\hline 192 & Vessel & $\begin{array}{l}\text { Pressurizer heater } \\
\text { sleeves }\end{array}$ & $\begin{array}{l}\text { RCS - reactor coolant } \\
\text { system }\end{array}$ & Cracking & PWSCC & Palo Verde 2 & $10 / 4 / 2000$ \\
\hline 188 & Vessel & $\begin{array}{l}\text { Pressurizer heater } \\
\text { sleeves }\end{array}$ & $\begin{array}{l}\text { RCS - reactor coolant } \\
\text { system }\end{array}$ & Cracking & PWSCC & Waterford 3 & $10 / 17 / 2000$ \\
\hline 96 & Vessel & Pressurizer nozzle & $\begin{array}{l}\text { RCS - reactor coolant } \\
\text { system }\end{array}$ & Cracking & PWSCC & Crystal River 3 & $10 / 4 / 2003$ \\
\hline 38 & Vessel & Pressurizer nozzle & $\begin{array}{l}\text { RCS - reactor coolant } \\
\text { system }\end{array}$ & Cracking & PWSCC & San Onofre 3 & $3 / 29 / 2006$ \\
\hline
\end{tabular}




\begin{tabular}{|c|c|c|c|c|c|c|c|c|c|}
\hline ID & COMPONENT & SUBCOMPONENT & \multicolumn{3}{|c|}{ SYSTEM } & AGING EFFECTS & AGING MECHANISMS & PLANT & $\begin{array}{l}\text { EVENT } \\
\text { DATE }\end{array}$ \\
\hline 94 & Vessel & Pressurizer sleeves & $\begin{array}{l}\mathrm{RCS} \\
\text { system }\end{array}$ & - reactor & coolant & Cracking & PWSCC & Millstone 2 & $10 / 11 / 2003$ \\
\hline 92 & Vessel & Pressurizer sleeves & $\begin{array}{l}\text { RCS } \\
\text { system }\end{array}$ & - reactor & coolant & Cracking & PWSCC & Waterford 3 & $10 / 26 / 2003$ \\
\hline 4 & Vessel & $\begin{array}{l}\text { Resistance } \\
\text { Temperature Detector }\end{array}$ & $\begin{array}{l}\text { RCS } \\
\text { system }\end{array}$ & - reactor & coolant & Leaking & $\begin{array}{l}\text { Mechanical joints not } \\
\text { intact }\end{array}$ & Turkey Point 3 & $9 / 8 / 2007$ \\
\hline 37 & Vessel & Sleeve & $\begin{array}{l}\text { RCS } \\
\text { system }\end{array}$ & - reactor & coolant & Cracking & IGSCC & Braidwood 1 & $4 / 25 / 2006$ \\
\hline 140 & Vessel & Sleeve & $\begin{array}{l}\text { RCS } \\
\text { system }\end{array}$ & - reactor & coolant & Cracking & PWSCC & Arkansas Nuclear 2 & $4 / 15 / 2002$ \\
\hline 69 & Vessel & Sleeve & $\begin{array}{l}\text { RCS } \\
\text { system }\end{array}$ & - reactor & coolant & Cracking & PWSCC & Arkansas Nuclear 2 & $3 / 9 / 2005$ \\
\hline 64 & Vessel & Sleeve & $\begin{array}{l}\text { RCS } \\
\text { system }\end{array}$ & - reactor & coolant & Cracking & PWSCC & Millstone 2 & $4 / 10 / 2005$ \\
\hline 110 & Vessel & Sleeve & $\begin{array}{l}\text { RCS } \\
\text { system }\end{array}$ & - reactor & coolant & Cracking & PWSCC & Palo Verde 3 & $3 / 29 / 2003$ \\
\hline 84 & Vessel & Sleeve & $\begin{array}{l}\text { RCS } \\
\text { system }\end{array}$ & - reactor & coolant & Cracking & PWSCC & Palo Verde 3 & $2 / 29 / 2004$ \\
\hline 62 & Vessel & Sleeve & $\begin{array}{l}\text { RCS } \\
\text { system }\end{array}$ & - reactor & coolant & Cracking & PWSCC & Waterford 3 & 4/19/2005 \\
\hline
\end{tabular}


Table 3-3 Degradation Occurrence Records (Secondary Information)

\begin{tabular}{|c|c|c|c|c|c|c|c|c|}
\hline ID & COMPONENT & SUBCOMPONENT & $\begin{array}{c}\text { HOW } \\
\text { IDENTIFIED }\end{array}$ & $\begin{array}{l}\text { EVALUATION } \\
\text { METHOD }\end{array}$ & REPAIR METHOD & DOCKET & REF & ML NUMBER \\
\hline 1 & Tank & $\begin{array}{l}\text { Tank shell - sensor } \\
\text { connection }\end{array}$ & Maitenance & Visual & Replacement & 5000237 & LER 07-001-01 & ML080910066 \\
\hline 2 & Piping system & Piping & $\begin{array}{l}\text { Cleaning of } \\
\text { corrosion }\end{array}$ & Visual & Replacement & 5000454 & LER 07-002-01 & ML080660544 \\
\hline 3 & Piping system & Piping & $\begin{array}{l}\text { Cleaning of } \\
\text { corrosion }\end{array}$ & Visual & Replacement & 5000455 & LER 07-002-01 & ML080660544 \\
\hline 4 & Vessel & $\begin{array}{l}\text { Resistance } \\
\text { Temperature Detector }\end{array}$ & $\begin{array}{l}\text { Refueling } \\
\text { outage }\end{array}$ & Visual & Replacement & 5000250 & LER 07-004-01 & ML080720677 \\
\hline 5 & Piping system & $\begin{array}{l}\text { nozzle-to-elbow } \\
\text { dissimilar metal butt } \\
\text { weld }\end{array}$ & $\begin{array}{l}\text { Refueling } \\
\text { outage }\end{array}$ & Visual & Repair & 5000346 & LER 08-001-00 & ML080640204 \\
\hline 6 & Piping system & $\begin{array}{l}\text { Reactor coolant pump } \\
\text { seal weld }\end{array}$ & Maintenance & $\begin{array}{l}\text { Visual I Liquid } \\
\text { penetrant }\end{array}$ & Replacement of the seal package & 5000389 & LER 07-002-00 & ML080580311 \\
\hline 7 & Piping system & Piping & $\begin{array}{l}\text { Maintenance/Vis } \\
\text { ual }\end{array}$ & Visual & Replacement & 5000296 & LER 07-004-00 & ML080290046 \\
\hline 8 & Exchanger & Tube plug & $\begin{array}{l}\text { Soidium level } \\
\text { high in steam } \\
\text { generator }\end{array}$ & N/A & $\begin{array}{l}\text { Replaced/ Will determine for } \\
\text { alternative (material) tube plug }\end{array}$ & 5000529 & LER 07-003-00 & ML073480125 \\
\hline 9 & Piping system & $\begin{array}{l}\text { Small bore piping \& } \\
\text { tubing }\end{array}$ & Video Monitoring & Visual & $\begin{array}{l}\text { Replacement of fretted EHC tubing } \\
\text { and installed a wood isolation block }\end{array}$ & 5000259 & LER 07-008-00 & ML073090091 \\
\hline 10 & Piping system & Socket weld fitting & $\begin{array}{l}\text { Unidentified } \\
\text { leakage/walk } \\
\text { down }\end{array}$ & Visual & Repalce seal injection piping & 5000389 & LER 07-001-00 & ML072970529 \\
\hline 11 & Piping system & Piping/Elbow & Visual & Visual & Replacement & 5000237 & LER 07-003-00 & ML072750663 \\
\hline 12 & Piping system & Snubber & Test & visual & replacement & 5000255 & LER 07-006-00 & ML072500072 \\
\hline 13 & Piping system & Hose & Alarms & Visual & Replacement & 5000461 & LER 07-003-00 & ML072350078 \\
\hline 14 & Piping system & Fitting & $\begin{array}{l}\text { Alarms } \\
\text { indicating an } \\
\text { EHC leak }\end{array}$ & Visual & Replacement of tube & 5000259 & LER 07-002-00 & ML072040345 \\
\hline 15 & Vessel & Nozzle weld & Repair & Ultra-sonic testing & A full structural weld overlay & 5000293 & LER 07-003-00 & ML071840196 \\
\hline
\end{tabular}




\begin{tabular}{|c|c|c|c|c|c|c|c|c|}
\hline ID & COMPONENT & SUBCOMPONENT & $\begin{array}{c}\text { HOW } \\
\text { IDENTIFIED }\end{array}$ & $\begin{array}{l}\text { EVALUATION } \\
\text { METHOD }\end{array}$ & REPAIR METHOD & DOCKET & REF & ML NUMBER \\
\hline 16 & RPV & Nozzle & $\begin{array}{l}\text { Inspection at } \\
\text { refuel outage }\end{array}$ & $\begin{array}{lr}\text { Liquid r penetrant } \\
\text { test } & (\mathrm{PT}) \\
\text { examination } & \end{array}$ & Repair & 5000455 & LER 07-001-00 & ML071590211 \\
\hline 17 & Tank & $\begin{array}{l}\text { Filter tanks and internal } \\
\text { components }\end{array}$ & N.A. & Inspection & Replaced & 5000305 & LER 06-005-00 & ML071640378 \\
\hline 18 & Piping system & Socket weld & Leaking & Visual & Replaced & 5000366 & LER 07-004-00 & ML071230404 \\
\hline 19 & Piping system & Piping & N/A & Visual & Replacement & 5000249 & LER 07-001-00 & ML071280263 \\
\hline 20 & Piping system & Piping & $\begin{array}{l}\text { Pressure } \\
\text { controller failed }\end{array}$ & Troubleshooting & Debris removed & 5000265 & LER 07-001-00 & ML071280295 \\
\hline 21 & RPV & Nozzle weld & $\begin{array}{l}\text { scheduled } \\
\text { examination }\end{array}$ & $\begin{array}{l}\text { UT } \quad \text { Ultrasonic } \\
\text { test }\end{array}$ & $\begin{array}{l}\text { overlay flawed weld with material } \\
\text { resistant to SCC }\end{array}$ & 5000331 & LER 07-003-00 & ML071150319 \\
\hline 22 & Exchanger & Heat exchanger & $\begin{array}{l}\text { NRC Final } \\
\text { significance } \\
\text { determination } \\
\text { letter }\end{array}$ & N.A. & Cleaned & 5000529 & LER 06-006-01 & ML070950342 \\
\hline 23 & Containment & $\begin{array}{l}\text { CAC cooling coil / } \\
\text { fitting }\end{array}$ & leaking & visual & $\begin{array}{l}\text { Plugging tubes to isolate the } \mathrm{H} \text { - } \\
\text { bend flaw }\end{array}$ & 5000255 & LER 07-002-00 & ML070871046 \\
\hline 24 & Exchanger & Exchanger & Leaking & N.A. & Replacement & 5000317 & LER 07-001-00 & ML070810509 \\
\hline 25 & Containment & $\begin{array}{l}\text { CAC cooling coil / } \\
\text { fitting }\end{array}$ & Leaking & Visual & $\begin{array}{l}\text { plugging tubes to isolate the } \mathrm{H} \text {-bend } \\
\text { flaw }\end{array}$ & 5000255 & LER 06-008-00 & ML070370354 \\
\hline 26 & Piping system & Fitting & $\begin{array}{l}\text { Failed to } \\
\text { maintain control } \\
\text { room } \\
\text { temperature }\end{array}$ & Visual & Repaired & 5000237 & $\begin{array}{l}\text { LER 2006-005- } \\
00\end{array}$ & ML070170356 \\
\hline 27 & Containment & $\begin{array}{l}\text { CAC cooling coil / } \\
\text { fitting }\end{array}$ & Leaking & Visual & $\begin{array}{l}\text { Installing blanks on the inlet and } \\
\text { outlet flanges of the cooling coil }\end{array}$ & 5000255 & LER 06-006-00 & ML063610189 \\
\hline 28 & Tank & SLC tank & Leaking & Visual & Remove source of chemical & 5000254 & LER 06-004-00 & ML063530355 \\
\hline 29 & Vessel & Nozzle-safe end weld & $\begin{array}{l}\text { In-service } \\
\text { examination }\end{array}$ & NA & Weld overlay & 5000482 & LER 06-003-00 & ML063490047 \\
\hline 30 & Piping system & Elbow & Leaking & Visual & Replacement & 5000277 & LER 06-003-00 & ML063420059 \\
\hline
\end{tabular}




\begin{tabular}{|c|c|c|c|c|c|c|c|c|}
\hline ID & COMPONENT & SUBCOMPONENT & $\begin{array}{c}\text { HOW } \\
\text { IDENTIFIED } \\
\end{array}$ & $\begin{array}{l}\text { EVALUATION } \\
\text { METHOD }\end{array}$ & REPAIR METHOD & DOCKET & REF & ML NUMBER \\
\hline 31 & RPV & Piping & $\begin{array}{l}\text { In-service } \\
\text { examination }\end{array}$ & NA & Removed and repaired by welding & 5000529 & LER 06-005-00 & ML063450132 \\
\hline 32 & Piping system & Piping & air in-leakage & Boroscope & Plugged and capped & 5000346 & LER 06-003-00 & ML063120173 \\
\hline 33 & Piping system & Fitting & Leaking & NA & Repaired & 5000305 & LER 06-009-01 & ML071140152 \\
\hline 34 & Piping system & Cooling coil & Leaking & Visual & Repair & 5000331 & LER 06-003-00 & ML062490486 \\
\hline 35 & Piping system & Valve-piping weld & Leaking & Visual & NA & 5000220 & LER 06-001-00 & ML062290262 \\
\hline 36 & Filter & Sealant (RTV) & Scheduled test & $\begin{array}{l}\text { Penetration and } \\
\text { system bypass test }\end{array}$ & $\begin{array}{l}\text { Applying new RTV over degraded } \\
\text { RTV }\end{array}$ & 5000266 & LER 06-001-00 & ML062200498 \\
\hline 37 & Vessel & Sleeve & Leaking & $\begin{array}{l}\text { Failure analysis } \\
\text { throught test }\end{array}$ & Plug and seal weld & 5000456 & LER 06-001-01 & ML062000190 \\
\hline 38 & Vessel & Pressurizer nozzle & Inspection & Visual & Repaired with Inconel 690 material & 5000362 & LER 06-003-00 & ML061500428 \\
\hline 39 & Piping system & Nozzle-to-elbow weld & $\begin{array}{l}\text { Planed } \\
\text { examination }\end{array}$ & Ultrasonic exam & A full structural weld overlay & 5000346 & LER 06-002-00 & ML061440286 \\
\hline 40 & Exchanger & $\begin{array}{l}\text { Small bore piping \& } \\
\text { tubing }\end{array}$ & Refueling & $\begin{array}{ll}\text { Eddy } \quad \text { current } \\
\text { testing }\end{array}$ & Removed from service by plugging & 5000323 & LER 06-002-00 & ML061450136 \\
\hline 41 & RPV & Piping weld & $\begin{array}{l}\text { Visual } \\
\text { examination } \\
\text { during refuel }\end{array}$ & Ultrasonic test & $\begin{array}{l}\text { Structurally replace the weld with a } \\
\text { clamp }\end{array}$ & 5000325 & LER 06-002-00 & ML061530280 \\
\hline 42 & RPV & Sleeve & $\begin{array}{l}\text { Examingation } \\
\text { with reactor } \\
\text { defueled }\end{array}$ & $\begin{array}{l}\text { Borescope } \\
\text { examination }\end{array}$ & Replaced & 5000346 & LER 02-009-02 & ML061420150 \\
\hline 43 & Filter & Damper & $\begin{array}{l}\text { Post } \\
\text { maintenance } \\
\text { testing }\end{array}$ & N.A. & Repair & 5000483 & LER 06-003-00 & ML061360329 \\
\hline 44 & Piping system & Piping weld & $\begin{array}{l}\text { Increase in } \\
\text { radioactivity }\end{array}$ & Robotic camera & Replaced & 5000425 & LER 06-001-00 & ML060960448 \\
\hline 45 & Exchanger & $\begin{array}{l}\text { Heat exchanger / Soft } \\
\text { iron gasket }\end{array}$ & Leaking & N.A. & Iolated first and planned to repair & 5000260 & LER 05-004-01 & ML060930599 \\
\hline 46 & Piping system & Piping weld & Leaking & $\begin{array}{l}\text { Laboratory } \\
\text { analysis }\end{array}$ & Replacement & 5000361 & LER 06-001-00 & ML060680086 \\
\hline
\end{tabular}




\begin{tabular}{|c|c|c|c|c|c|c|c|c|}
\hline ID & COMPONENT & SUBCOMPONENT & $\begin{array}{c}\text { HOW } \\
\text { IDENTIFIED }\end{array}$ & $\begin{array}{l}\text { EVALUATION } \\
\text { METHOD }\end{array}$ & REPAIR METHOD & DOCKET & REF & ML NUMBER \\
\hline 47 & Containment & Torus & Leaking & $\begin{array}{l}\text { Lab analysis and } \\
\text { fracture mechanics } \\
\text { eval }\end{array}$ & $\begin{array}{l}\text { ASME code repair of the Torus } \\
\text { crack }\end{array}$ & 5000333 & LER 05-003-01 & ML053630257 \\
\hline 48 & Exchanger & CAC cooling coil & Leaking & NA & Plugging & 5000255 & LER 05-006-00 & ML053430217 \\
\hline 49 & Exchanger & $\begin{array}{l}\text { Small bore piping \& } \\
\text { tubing }\end{array}$ & Refueling & $\begin{array}{l}\text { Eddy current } \\
\text { testing }\end{array}$ & Removed from service by plugging & 5000275 & LER 05-001-00 & ML053410394 \\
\hline 50 & Exchanger & $\begin{array}{l}\text { Condensor / Small bore } \\
\text { piping \& tubing }\end{array}$ & Leaking & NA & $\begin{array}{l}\text { Remove the slop drain lines from } \\
\text { the condenser }\end{array}$ & 5000298 & LER 05-004-00 & ML053260429 \\
\hline 51 & Exchanger & Door & $\begin{array}{l}\text { Testing during } \\
\text { shutdown }\end{array}$ & NA & Cleaned and lubricated & 5000369 & LER 05-004-00 & ML053410422 \\
\hline 52 & Exchanger & $\begin{array}{l}\text { Small bore piping \& } \\
\text { tubing }\end{array}$ & EDG inoperable & NA & Reparied using a new fitting & 5000458 & LER 05-003-00 & ML053180172 \\
\hline 53 & Exchanger & Piping weld & NA & NA & Plugged & 5000251 & LER 04-004-01 & ML053180169 \\
\hline 54 & Exchanger & Piping weld & Hydrogen leak & NA & $\begin{array}{l}\text { Griding out the socket weld and re- } \\
\text { welding }\end{array}$ & 5000255 & LER 05-005-00 & ML053050420 \\
\hline 55 & Containment & $\begin{array}{l}\text { Small bore piping \& } \\
\text { tubing }\end{array}$ & $\begin{array}{l}\text { Drywell floor } \\
\text { draing leakage }\end{array}$ & Visual & Removed & 5000354 & LER 05-003-01 & ML052850369 \\
\hline 56 & Containment & Test cap & In-service test & NA & Installed a new cap & 5000219 & LER 05-003-00 & ML052630373 \\
\hline 57 & Exchanger & Piping & NA & NA & Repaired & 5000499 & LER 05-004-00 & ML052630031 \\
\hline 58 & Piping system & Piping weld & Leaking & Visual & $\begin{array}{l}\text { Repairment and Installation of a } \\
\text { shim plate }\end{array}$ & 5000333 & LER 05-004-00 & ML052510052 \\
\hline 59 & RPV & $\begin{array}{l}\text { CRDM - } \\
\begin{array}{l}\text { control rod } \\
\text { drive } \\
\text { (nozzle) }\end{array}\end{array}$ & Visual inspection & $\begin{array}{l}\text { Non-destrictive } \\
\text { testing (Eddy } \\
\text { current, ultrasonic, } \\
\text { dye penetrant) }\end{array}$ & Remove all crack and weld repair & 5000287 & LER 01-001-01 & ML052380420 \\
\hline 60 & RPV & Piping & $\begin{array}{l}\text { Preplanned in- } \\
\text { service } \\
\text { exmamination }\end{array}$ & $\begin{array}{l}\text { Eddy } \quad \text { current } \\
\text { testing }\end{array}$ & Machining & 5000529 & LER 05-001-00 & ML051880073 \\
\hline 61 & Tank & Piping weld & Leaking & NA & Replaced & 5000272 & LER 05-002-00 & ML051790154 \\
\hline 62 & Vessel & Sleeve & Leaking & $\begin{array}{l}\text { Visual inspection } \\
\text { during refueling }\end{array}$ & Repalcement & 5000382 & LER 05-001-00 & ML051710355 \\
\hline
\end{tabular}




\begin{tabular}{|c|c|c|c|c|c|c|c|c|}
\hline ID & COMPONENT & SUBCOMPONENT & $\begin{array}{c}\text { HOW } \\
\text { IDENTIFIED }\end{array}$ & $\begin{array}{l}\text { EVALUATION } \\
\text { METHOD }\end{array}$ & REPAIR METHOD & DOCKET & REF & ML NUMBER \\
\hline 63 & Exchanger & Piping weld & leaking & Visual & Machining and seal weld & 5000482 & LER 05-002-00 & ML051720389 \\
\hline 64 & Vessel & Sleeve & leaking & $\begin{array}{lr}\text { In-service } & \text { visual } \\
\text { inspection } & \text { during } \\
\text { refueling } & \\
\end{array}$ & $\begin{array}{l}\text { Encapsulates by a mechanical } \\
\text { nozzle seal assembly }\end{array}$ & 5000336 & LER 05-002-00 & ML051650242 \\
\hline 65 & Piping system & $\begin{array}{l}\text { Small bore piping \& } \\
\text { tubing }\end{array}$ & Leaking & Visual & Replaced & 5000311 & LER 05-002-00 & ML051650342 \\
\hline 66 & Piping system & Piping weld & Leaking & Visual & $\begin{array}{l}\text { Affected pipe shortened to avoid } \\
\text { resonance }\end{array}$ & 5000354 & LER 05-002-00 & ML051540027 \\
\hline 67 & Piping system & Piping & Leaking & Ultrasonic & Replaced & 5000483 & LER 05-002-00 & ML051460343 \\
\hline 68 & Piping system & Piping weld & Leakage test & Leakage test & Removed and weld a plug & 5000388 & LER 05-001-00 & ML051440352 \\
\hline 69 & Vessel & Sleeve & Visual inspection & NDE & $\begin{array}{l}\text { Applied mechanical nozzle seal } \\
\text { assemblies }\end{array}$ & 5000368 & LER 05-001-00 & ML051310236 \\
\hline 70 & Piping system & Nozzle & UT inspection & UT inspection & Overlay weld & 5000318 & LER 05-001-00 & ML051180015 \\
\hline 71 & Piping system & Piping weld & Walkdown & Walkdown & $\begin{array}{l}\text { Repaired and added tie-back } \\
\text { support }\end{array}$ & 5000389 & LER 05-001-00 & ML051050350 \\
\hline 72 & Piping system & Piping weld & Leakage & NA & Cutting off and plugging & 5000499 & $\begin{array}{l}\text { LER 1361-972- } \\
72\end{array}$ & ML050980111 \\
\hline 73 & Exchanger & Elbow & Leaking & NA & NA & 5000306 & LER 04-001-01 & ML050890314 \\
\hline 74 & RPV & 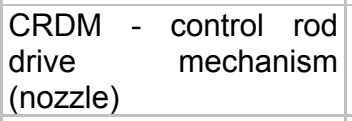 & Leaking & $\begin{array}{l}\text { Ultrasonic/dye } \\
\text { penetrant }\end{array}$ & Half-nozzle removed and replaced & 5000255 & LER 04-002-00 & ML043560278 \\
\hline 75 & Piping system & Piping weld & Steam leak & NA & Repaired & 5000354 & LER 04-010-00 & ML043560178 \\
\hline 76 & Piping system & Underground piping & Leaking & NA & Rerouted & 5000281 & LER 04-001-01 & ML043280416 \\
\hline 77 & Exchanger & Nozzle weld & $\begin{array}{l}\text { Exam during } \\
\text { refueling }\end{array}$ & Visual & $\begin{array}{l}\text { Machining and welding in a new } \\
\text { plug }\end{array}$ & 5000414 & LER 04-001-00 & ML043280501 \\
\hline 78 & Piping system & Nozzle & Leaking & NA & Replacement & 5000275 & LER 04-002-00 & ML042790449 \\
\hline
\end{tabular}




\begin{tabular}{|c|c|c|c|c|c|c|c|c|}
\hline ID & COMPONENT & SUBCOMPONENT & $\begin{array}{c}\text { HOW } \\
\text { IDENTIFIED }\end{array}$ & $\begin{array}{l}\text { EVALUATION } \\
\text { METHOD }\end{array}$ & REPAIR METHOD & DOCKET & REF & ML NUMBER \\
\hline 79 & RPV & \begin{tabular}{|lr}
$\begin{array}{l}\text { CRDM } \\
\text { drive } \\
\text { (nozzle) }\end{array}$ & $\begin{array}{l}\text { control rod } \\
\text { mechanism }\end{array}$ \\
\end{tabular} & $\begin{array}{l}\text { Inspection } \\
\text { during refueling } \\
\text { outage }\end{array}$ & Ultrasonic & $\begin{array}{l}\text { Inside diameter temper bead half- } \\
\text { nozzle weld }\end{array}$ & 5000313 & LER 04-002-00 & ML041830363 \\
\hline 80 & RPV & $\begin{array}{l}\text { CRDM - control rod } \\
\text { drive mechanism }\end{array}$ & $\begin{array}{l}\text { NDE as required } \\
\text { by the First } \\
\text { Revised NRC } \\
\text { Order }\end{array}$ & Ultrasonic & Weld & 5000266 & LER 04-001-00 & ML050870170 \\
\hline 81 & Piping system & Piping weld & Leaking & NA & Installed new nozzle & 5000395 & LER 04-001-00 & ML042740125 \\
\hline 82 & Exchanger & $\begin{array}{l}\text { Small bore piping \& } \\
\text { tubing }\end{array}$ & $\begin{array}{l}\text { Eddy current } \\
\text { testing during no } \\
\text { mode }\end{array}$ & $\begin{array}{l}\text { Eddy current } \\
\text { testing }\end{array}$ & Plugged & 5000275 & LER 04-001-00 & ML041400030 \\
\hline 83 & Piping system & Piping weld & $\begin{array}{l}\text { Scheduled } \\
\text { inservice } \\
\text { inspection }\end{array}$ & Ultrasonic & Overlay weld & 5000265 & LER 04-002-00 & ML041390526 \\
\hline 84 & Vessel & Sleeve & $\begin{array}{l}\text { Boric acid } \\
\text { walkdown }\end{array}$ & Visual & $\begin{array}{l}\text { Mechanical nozzle seal assembly } \\
\text { (MNSA) }\end{array}$ & 5000530 & LER 04-001-00 & ML041270485 \\
\hline 85 & Piping system & Piping weld & NA & NA & Overlay weld & 5000528 & LER 04-001-00 & ML041040027 \\
\hline 86 & Piping system & Fitting & Leaking & NA & New fitting installed & 5000269 & LER 04-001-00 & ML040760839 \\
\hline 87 & Exchanger & $\begin{array}{l}\text { Condenser (including } \\
\text { ice) \& supports }\end{array}$ & Air in-leakage & NA & Installed penetration weld & 5000331 & LER 03-006-00 & ML040340345 \\
\hline 88 & Piping system & Piping weld & Leaking & NA & Shortened and welded & 5000482 & LER 03-004-00 & ML040210791 \\
\hline 89 & Anchorage & Nut & NA & Visual & Replacement & 5000321 & LER 03-003-00 & ML040210327 \\
\hline 90 & Vessel & Pressurizer & $\begin{array}{l}\text { Inspection at } \\
\text { refueling outage }\end{array}$ & visual & Replaced & 5000289 & LER 03-003-00 & ML033580625 \\
\hline 91 & Piping system & Nozzle & $\begin{array}{l}\text { Leakage found } \\
\text { during refueling } \\
\text { outage }\end{array}$ & NA & Welded repair & 5000382 & LER 03-003-00 & ML033560242 \\
\hline 92 & Vessel & Pressurizer sleeves & $\begin{array}{l}\text { Leakage found } \\
\text { during refueling } \\
\text { outage }\end{array}$ & NA & Mechanical nozzle seal assemblies & 5000382 & LER 03-003-00 & ML033560242 \\
\hline 93 & Exchanger & Heat exchanger & Inspection & NA & $\begin{array}{l}\text { To be replaced at next refueling } \\
\text { outage }\end{array}$ & 5000413 & LER 03-006-00 & ML033500337 \\
\hline
\end{tabular}




\begin{tabular}{|c|c|c|c|c|c|c|c|c|}
\hline ID & COMPONENT & SUBCOMPONENT & $\begin{array}{c}\text { HOW } \\
\text { IDENTIFIED } \\
\end{array}$ & $\begin{array}{l}\text { EVALUATION } \\
\text { METHOD }\end{array}$ & REPAIR METHOD & DOCKET & REF & ML NUMBER \\
\hline 94 & Vessel & Pressurizer sleeves & Inspection & Visual & Mechanical nozzle seal assemblies & 5000336 & LER 03-004-00 & ML033460378 \\
\hline 95 & RPV & Nozzle-to-cap weld & $\begin{array}{l}\text { Planned } \\
\text { inspection }\end{array}$ & Visual & Automated structural weld overlay & 5000293 & LER 03-006-00 & ML033360733 \\
\hline 96 & Vessel & Pressurizer nozzle & Inspection & Visual & "Halfnozzle" technique & 5000302 & LER 03-003-00 & ML033320052 \\
\hline 97 & Vessel & $\begin{array}{l}\text { Pressurizer - filters and } \\
\text { screens }\end{array}$ & NA & NA & Replaced & 5000346 & LER 98-002-01 & ML033170198 \\
\hline 98 & Filter & Screens & Fish impinging & NA & Replaced & 5000315 & LER 03-003-01 & ML033180115 \\
\hline 99 & RPV & $\begin{array}{ll}\begin{array}{l}\text { CRDM } \\
\text { drive } \\
\text { (nozzle) }\end{array} & \begin{array}{l}\text { control rod } \\
\text { mechanism }\end{array} \\
\end{array}$ & $\begin{array}{l}\text { Scheduled } \\
\text { inspection at } \\
\text { refueling outage }\end{array}$ & Visual & $\begin{array}{l}\text { Reactor vessel head retired and } \\
\text { replaced }\end{array}$ & 5000269 & LER 03-002-00 & ML033090486 \\
\hline 100 & RPV & Nozzle & Leaking & Ultrosonic & Half-nozzles and weld & 5000498 & LER 03-003-01 & ML032950483 \\
\hline 101 & RPV & Steam dryer & Inspection & NA & Repair (no detail) & 5000265 & LER 03-004-00 & ML032461172 \\
\hline 102 & RPV & Vent line & Leaking & Ultrasonic & Replaced & 5000254 & LER 03-001-00 & ML032120510 \\
\hline 103 & Exchanger & Heat exchanger & NA & NA & Chemically cleaned & 5000413 & LER 03-004-00 & ML031970061 \\
\hline 104 & Piping system & $\begin{array}{l}\text { Small bore piping \& } \\
\text { tubing }\end{array}$ & Leaking & NA & $\begin{array}{l}\text { Modified to increase tubing wall } \\
\text { thickness }\end{array}$ & 5000458 & LER 03-001-01 & ML031820539 \\
\hline 105 & RPV & Nozzle & $\begin{array}{l}\text { Inspection at } \\
\text { refuleling outage }\end{array}$ & Ultrasonic & $\begin{array}{l}\text { Removed lower portion and } \\
\text { relocated boundary weld }\end{array}$ & 5000389 & LER 03-002-00 & ML031900041 \\
\hline 106 & Filter & $\begin{array}{l}\text { TWS - traveling water } \\
\text { screen }\end{array}$ & $\begin{array}{l}\text { Main feed pump } \\
\text { condenser } \\
\text { fouling by fish }\end{array}$ & NA & $\begin{array}{l}\text { Replaced with stainless steel } \\
\text { panels }\end{array}$ & 5000315 & LER 03-003-00 & ML031820557 \\
\hline 107 & Piping system & Piping & Leaking & Visual & Replaced & 5000331 & LER 03-004-00 & ML031760687 \\
\hline 108 & Exchanger & Condensor / piping & Air in-leakage & NA & Replaced during refueling outage & 5000389 & LER 03-001-00 & ML031550064 \\
\hline 109 & Piping system & Nozzle & $\begin{array}{l}\text { Leakage found } \\
\text { during refueling } \\
\text { outage }\end{array}$ & NA & Replaced & 5000530 & LER 03-002-00 & ML031540552 \\
\hline
\end{tabular}




\begin{tabular}{|c|c|c|c|c|c|c|c|c|}
\hline ID & COMPONENT & SUBCOMPONENT & $\begin{array}{c}\text { HOW } \\
\text { IDENTIFIED } \\
\end{array}$ & $\begin{array}{l}\text { EVALUATION } \\
\text { METHOD }\end{array}$ & REPAIR METHOD & DOCKET & REF & ML NUMBER \\
\hline 110 & Vessel & Sleeve & $\begin{array}{l}\text { Leakage found } \\
\text { during refueling } \\
\text { outage }\end{array}$ & NA & Replaced & 5000530 & $\begin{array}{ll}\text { LER } & \text { 03-002- } \\
\text { OOA } & \end{array}$ & ML031540552 \\
\hline 111 & Piping system & $\begin{array}{l}\text { Small bore piping \& } \\
\text { tubing }\end{array}$ & NA & NA & $\begin{array}{l}\text { Replaced and added additional } \\
\text { supports }\end{array}$ & 5000277 & LER 03-001-00 & ML031490372 \\
\hline 112 & RPV & 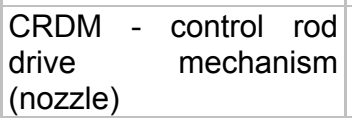 & $\begin{array}{l}\text { Inspection at } \\
\text { refueling outage }\end{array}$ & Visual & Reactor vessel head retired & 5000287 & LER 03-001-00 & ML031490044 \\
\hline 113 & Exchanger & Condensor / tubing & Leaking & NA & Replaced & 5000528 & LER 03-002-00 & ML031400058 \\
\hline 114 & Other & $\begin{array}{ll}\begin{array}{l}\text { Gypsum } \\
\text { assembly }\end{array} & \text { board }\end{array}$ & Waldown & Visual & Cosmetically repaired & 5000263 & LER 03-001-00 & ML031400643 \\
\hline 115 & Exchanger & CAC components & NA & NA & Refurbishment/replacement & 5000346 & LER 02-008-01 & ML031330192 \\
\hline 116 & Piping system & Piping & NA & NA & Refurbishment/replacement & 5000346 & $\begin{array}{l}\text { LER } \\
\text { 01A }\end{array}$ & ML031330192 \\
\hline 117 & Exchanger & $\begin{array}{l}\text { Steam generator } \\
\text { tubing }\end{array}$ & $\begin{array}{l}\text { Scheduled } \\
\text { surveillance at } \\
\text { refueling outage }\end{array}$ & $\begin{array}{l}\text { Eddy current ply } \\
\text { point testing data }\end{array}$ & Plugging or sleeving & 5000445 & LER 02-002-01 & ML031210481 \\
\hline 118 & RPV & Penetration & $\begin{array}{l}\text { Inspection at } \\
\text { refueling outage }\end{array}$ & Visual & Reactor head replaced & 5000338 & LER 03-001-00 & ML031200697 \\
\hline 119 & Exchanger & Steam deflector plate & $\begin{array}{l}\text { Conductivity } \\
\text { alarm }\end{array}$ & NA & $\begin{array}{l}\text { Broken deflector plate replaced and } \\
\text { repaired valve and plugged tubes }\end{array}$ & 5000331 & LER 03-001-00 & ML030920458 \\
\hline 120 & Exchanger & $\begin{array}{l}\text { Steam generator } \\
\text { tubing }\end{array}$ & Inspection & Eddy current & Plugging & 5000323 & LER 03-001-00 & ML030780463 \\
\hline 121 & Exchanger & $\begin{array}{l}\text { Steam generator } \\
\text { tubing }\end{array}$ & Inspection & Eddy current & Plugging & 5000323 & LER 03-001-00 & ML030780463 \\
\hline 122 & Piping system & Support & NA & NA & Repaired & 5000249 & LER 02-005-01 & ML030590240 \\
\hline 123 & Piping system & Piping weld & Leaking & NA & $\begin{array}{l}\text { Minimized welding residual stress } \\
\text { and eleminated mechanically } \\
\text { induced stress }\end{array}$ & 5000249 & LER 02-006-00 & ML030430376 \\
\hline 124 & Piping system & Thermal sleeve & $\begin{array}{l}\text { Examination } \\
\text { with reactor } \\
\text { defueled }\end{array}$ & Borescope & Replaced & 5000346 & LER 02-009-00 & ML030370013 \\
\hline 125 & Other & Static line hanger & NA & NA & Replaced & 5000255 & LER 02-002-00 & ML030300342 \\
\hline
\end{tabular}




\begin{tabular}{|c|c|c|c|c|c|c|c|c|}
\hline ID & COMPONENT & SUBCOMPONENT & $\begin{array}{c}\text { HOW } \\
\text { IDENTIFIED }\end{array}$ & $\begin{array}{l}\text { EVALUATION } \\
\text { METHOD }\end{array}$ & REPAIR METHOD & DOCKET & REF & ML NUMBER \\
\hline 126 & Exchanger & $\begin{array}{lll}\begin{array}{l}\text { Steam } \\
\text { tubing }\end{array} & \text { generator }\end{array}$ & $\begin{array}{l}\text { In-situ pressure } \\
\text { testing }\end{array}$ & $\begin{array}{l}\text { In-situ pressure } \\
\text { testing }\end{array}$ & Plugging & 5000270 & LER 02-003-00 & ML023600191 \\
\hline 127 & Piping system & Piping weld & Leaking & NA & Replaced & 5000249 & LER 02-003-00 & ML023520043 \\
\hline 128 & RPV & $\begin{array}{ll}\text { CRDM } & \text { - control rod } \\
\text { drive } & \text { mechanism } \\
\text { (nozzle) } & \end{array}$ & $\begin{array}{l}\text { Inspection at } \\
\text { refueling outage }\end{array}$ & Visual & $\begin{array}{l}\text { Partially removed and applied new } \\
\text { weld }\end{array}$ & 5000313 & LER 02-003-00 & ML023400549 \\
\hline 129 & Piping system & Piping weld & $\begin{array}{l}\text { Inspection at } \\
\text { refueling outage }\end{array}$ & NA & $\begin{array}{l}\text { Replaced with an enhanced } \\
\text { configuration }\end{array}$ & 5000313 & LER 02-004-00 & ML023400485 \\
\hline 130 & RPV & $\begin{array}{l}\begin{array}{l}\text { CRDM } \\
\text { drive } \\
\text { (nozzle) }\end{array} \\
\text { mechanism } \\
\end{array}$ & \begin{tabular}{ll}
\multicolumn{2}{l}{ Required } \\
inspection per \\
NRC $\quad$ Bulletin \\
$2001-01$
\end{tabular} & Visual & Repair (no detail) & 5000270 & LER 02-002-00 & ML023470024 \\
\hline 131 & Exchanger & $\begin{array}{l}\text { Steam generator - } \\
\text { tubing }\end{array}$ & $\begin{array}{l}\text { Inspection at } \\
\text { refueling outage }\end{array}$ & NA & Plugged & 5000483 & $\begin{array}{l}\text { LER 2002-011- } \\
00\end{array}$ & ML023310226 \\
\hline 132 & RPV & Penetration & $\begin{array}{l}\text { Inspection at } \\
\text { refueling outage }\end{array}$ & Visual + NDE & Replacement of RPV head & 5000339 & LER 02-001-00 & ML023180480 \\
\hline 133 & Piping system & Piping weld & $\begin{array}{l}\text { Increasing } \\
\text { radiation trend }\end{array}$ & Visual & Affected welds and elbows replaced & 5000336 & LER 02-004-00 & ML022840184 \\
\hline 134 & Piping system & Piping weld & Low level alarm & NA & NA & 5000317 & LER 02-003-00 & ML022630111 \\
\hline 135 & RPV & Steam dryer & NA & NA & Replaced with thicker plate & 5000265 & LER 02-003-00 & ML022610332 \\
\hline 136 & Concrete & Floor & Leaking & NA & Seal & 5000397 & LER 02-003-00 & ML022270273 \\
\hline 137 & Piping system & Piping weld & Leaking & $\begin{array}{l}\text { RT - Radiographic } \\
\text { testing }\end{array}$ & $\begin{array}{l}\text { Grinding out degraded area and } \\
\text { weld buildup }\end{array}$ & 5000286 & LER 02-001-00 & ML022000155 \\
\hline 138 & Exchanger & $\begin{array}{l}\text { Small bore piping \& } \\
\text { tubing }\end{array}$ & Leaking & $\begin{array}{l}\text { Laboratory } \\
\text { analysis }\end{array}$ & Installed sleeves & 5000305 & LER 02-002-00 & ML021920465 \\
\hline 139 & Piping system & Piping & Leaking & $\begin{array}{l}\text { Scanning electron } \\
\text { microscope }\end{array}$ & Replaced & 5000281 & LER 02-001-00 & ML021710180 \\
\hline 140 & Vessel & Sleeve & Leaking & Visual & Mechanical Nozzle Seal Assembly & 5000368 & LER 02-001-00 & ML021680047 \\
\hline 141 & RPV & Nozzle & $\begin{array}{l}\text { Inspection at } \\
\text { refueling outage }\end{array}$ & Ultrasonic & NA & 5000269 & LER 02-003-00 & ML021570019 \\
\hline
\end{tabular}




\begin{tabular}{|c|c|c|c|c|c|c|c|c|}
\hline ID & COMPONENT & SUBCOMPONENT & $\begin{array}{c}\text { HOW } \\
\text { IDENTIFIED }\end{array}$ & $\begin{array}{l}\text { EVALUATION } \\
\text { METHOD }\end{array}$ & REPAIR METHOD & DOCKET & REF & ML NUMBER \\
\hline 142 & Exchanger & $\begin{array}{ll}\begin{array}{l}\text { Steam } \\
\text { tubing }\end{array} & \text { generator } \quad- \\
\end{array}$ & $\begin{array}{ll}\text { Eddy } & \text { current } \\
\text { testing } & \end{array}$ & $\begin{array}{ll}\text { Eddy } & \text { current } \\
\text { testing } & \end{array}$ & Plugged & 5000275 & LER 02-002-00 & ML021560548 \\
\hline 143 & RPV & $\begin{array}{l}\text { CRDM - control rod } \\
\text { drive } \\
\text { (nozzle) }\end{array}$ & Ultrosonic exam & Ultrosonic exam & NA & 5000346 & LER 02-002-00 & ML021220082 \\
\hline 144 & Vessel & $\begin{array}{l}\text { Pressurizer - heater } \\
\text { sleeve penetrations }\end{array}$ & Leaking & Visual & Mechanical Nozzle Seal Assembly & 5000336 & LER 02-001-00 & ML021210508 \\
\hline 145 & RPV & CRDM - upper housing & Leaking & NDE & Replaced & 5000255 & LER 01-004-01 & ML020870353 \\
\hline 146 & Piping system & $\begin{array}{l}\text { Small bore piping \& } \\
\text { tubing }\end{array}$ & Degraded flow & NA & Cleaned & 5000412 & LER 02-001-00 & ML020710575 \\
\hline 147 & Tank & Floating cover & $\begin{array}{l}\text { Failure of motor } \\
\text { driven auxiliary } \\
\text { feedwater pump }\end{array}$ & NA & CST cover removed & 5000483 & LER 02-001-01 & ML020720446 \\
\hline 148 & RPV & 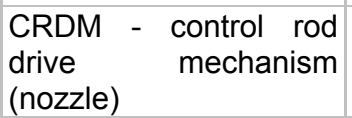 & $\begin{array}{lr}\text { Visual inspection } \\
\text { at } & \text { refueling } \\
\text { outage } & \end{array}$ & Ultrasonic & Repaired & 5000287 & LER 01-003-00 & ML020350290 \\
\hline 149 & Piping system & Pipe-to-elbow weld & Leaking & Visual & Replacement of elbow & 5000247 & LER 01-006-00 & ML020090594 \\
\hline 150 & Piping system & $\begin{array}{l}\text { Small bore piping \& } \\
\text { tubing }\end{array}$ & Pump noise & Visual & Replaced & 5000271 & LER 01-005-00 & ML020240466 \\
\hline 151 & RPV & CRDM penetration & $\begin{array}{l}\text { Inspection at } \\
\text { refueling outage }\end{array}$ & Visual & Repaired & 5000280 & LER 01-003-00 & ML020520345 \\
\hline 152 & Piping system & Piping weld & Leaking & $\begin{array}{l}\text { Radiographic } \\
\text { examination }\end{array}$ & Weld removed and replaced & 5000354 & LER 01-006-00 & ML020220237 \\
\hline 153 & RPV & 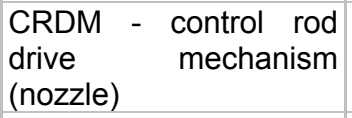 & Leaking & $\begin{array}{l}\text { Liquid penetration } \\
\text { test }\end{array}$ & Machined and applied new weld & 5000289 & LER 01-002-00 & ML020160399 \\
\hline 154 & RPV & $\begin{array}{l}\text { Thermocouple (T/C) } \\
\text { nozzles }\end{array}$ & Leaking & $\begin{array}{l}\text { Liquid penetration } \\
\text { test }\end{array}$ & Plugged & 5000289 & $\begin{array}{l}\text { LER 01-002- } \\
\text { OOA }\end{array}$ & ML020160399 \\
\hline 155 & Exchanger & Heat exchanger & $\begin{array}{l}\text { Elevated } \\
\text { radionuclide } \\
\text { activity in SW }\end{array}$ & NA & Isolated using plugs & 5000302 & LER 01-003-00 & ML020430199 \\
\hline 156 & RPV & $\begin{array}{l}\begin{array}{l}\text { CRDM } \\
\text { drive } \\
\text { (nozzle) }\end{array} \\
\text { mechanism } \\
\end{array}$ & Visual inspection & Ultrasonic & $\begin{array}{l}\text { Ambient temperature temper bead } \\
\text { repair technique }\end{array}$ & 5000302 & LER 01-004-00 & ML020160225 \\
\hline 157 & Exchanger & $\begin{array}{l}\text { Steam generator } \\
\text { nozzle to vessel weld }\end{array}$ & $\begin{array}{l}\text { Walk down at } \\
\text { refueling outage }\end{array}$ & $\begin{array}{l}\text { Visual and } \\
\text { penetrant test }\end{array}$ & $\begin{array}{l}\text { Removed old weld (Alloy 600) and } \\
\text { applied new weld (Inconel 52/152) }\end{array}$ & 5000414 & $\begin{array}{l}\text { LER 803-831- } \\
36\end{array}$ & ML020030237 \\
\hline
\end{tabular}




\begin{tabular}{|c|c|c|c|c|c|c|c|c|}
\hline ID & COMPONENT & SUBCOMPONENT & $\begin{array}{c}\text { HOW } \\
\text { IDENTIFIED }\end{array}$ & $\begin{array}{l}\text { EVALUATION } \\
\text { METHOD }\end{array}$ & REPAIR METHOD & DOCKET & REF & ML NUMBER \\
\hline 158 & Piping system & Drain trap outlet plug & Steam leaking & NA & Replaced & 5000331 & LER 01-004-00 & ML020310457 \\
\hline 159 & Structural steel & Door & $\begin{array}{l}\text { Airlock door } \\
\text { failed during } \\
\text { removal of plant } \\
\text { equipment }\end{array}$ & Visual & Repaired & 5000316 & $\begin{array}{l}\text { LER 2001-002- } \\
01\end{array}$ & ML020240312 \\
\hline 160 & Filter & Strainer basket & Surveillance test & NA & Replaced & 5000316 & LER 01-003-00 & ML020220028 \\
\hline 161 & Filter & Strainer basket & Surveillance test & NA & Replaced & 5000315 & LER 01-003-00 & ML020220028 \\
\hline 162 & RPV & Feedwater nozzle & $\begin{array}{l}\text { Out of } \\
\text { calibration }\end{array}$ & NA & NA & 5000271 & LER 01-004-00 & ML013650369 \\
\hline 163 & Piping system & Cooling coil & Leaking & NA & Replacement & 5000271 & LER 20012009 & ML070670447 \\
\hline 164 & Other & Flood panel & $\begin{array}{l}\text { Preventive } \\
\text { maintenance }\end{array}$ & NA & Repair & 5000282 & LER 01-003-00 & ML012610162 \\
\hline 165 & Filter & Screens & $\begin{array}{ll}\text { Water level } \\
\text { decreased }\end{array}$ & NA & NA & 5000301 & LER 01-002-00 & ML012330129 \\
\hline 166 & Structural steel & Door & NA & NA & Weld buildup & 5000336 & LER 01-006-00 & ML012320075 \\
\hline 167 & Exchanger & Radiator cooling fin & Leaking & NA & Replacement of radiator & 5000335 & LER 01-006-00 & ML012050195 \\
\hline 168 & RPV & $\begin{array}{l}\text { CRDM - control rod } \\
\begin{array}{l}\text { drive } \\
\text { (nozzle) }\end{array}\end{array}$ & $\begin{array}{lr}\text { Visual inspection } \\
\text { at } & \text { refueling } \\
\text { outage } & \end{array}$ & Dye-penetrant test & Machining and weld & 5000270 & LER 01-002-00 & ML011830021 \\
\hline 169 & Piping system & Nozzle & Leaking & Visual & Replacement & 5000335 & LER 01-003-00 & ML011700213 \\
\hline 170 & RPV & CRDM Seal housing & $\begin{array}{l}\text { Inspection at } \\
\text { refueling outage }\end{array}$ & NA & Installed new Inconel housing & 5000255 & $\begin{array}{l}\text { LER 001-002- } \\
00\end{array}$ & ML011560030 \\
\hline 171 & Structural steel & Bolt & $\begin{array}{l}\text { Pump } \\
\text { inoperable }\end{array}$ & NA & Tightened & 5000289 & LER 01-001-01 & ML011500423 \\
\hline 172 & Piping system & Nozzle & $\begin{array}{l}\text { Inspection at } \\
\text { refueling outage }\end{array}$ & $\begin{array}{ll}\text { Eddy } & \text { current } \\
\text { testing } & \end{array}$ & Cut and plug & 5000528 & LER 01-001-00 & ML011500148 \\
\hline 173 & Exchanger & $\begin{array}{l}\text { Steam generator } \\
\text { tubing }\end{array}$ & $\begin{array}{l}\text { Inspection at } \\
\text { refueling outage }\end{array}$ & Eddy current exam & Plugging & 5000499 & LER 01-003-00 & ML011420313 \\
\hline
\end{tabular}




\begin{tabular}{|c|c|c|c|c|c|c|c|c|}
\hline ID & COMPONENT & SUBCOMPONENT & $\begin{array}{c}\text { HOW } \\
\text { IDENTIFIED }\end{array}$ & $\begin{array}{l}\text { EVALUATION } \\
\text { METHOD }\end{array}$ & REPAIR METHOD & DOCKET & REF & ML NUMBER \\
\hline 174 & RPV & 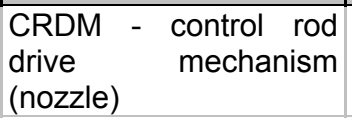 & \begin{tabular}{|lr|}
\multicolumn{2}{|l|}{ Visual inpsection } \\
at & refueling \\
outage &
\end{tabular} & $\begin{array}{l}\text { Liquid penetrant } \\
\text { exam }\end{array}$ & Embedded flaw repair & 5000313 & LER 01-002-00 & ML011350195 \\
\hline 175 & RPV & $\begin{array}{l}\begin{array}{l}\text { CRDM } \\
\text { drive }\end{array} \\
\text { (nozzle) }\end{array}$ & $\begin{array}{l}\text { Visual inspection } \\
\text { at planned } \\
\text { maintenance } \\
\text { outage }\end{array}$ & $\begin{array}{l}\text { Eddy current } \\
\text { examination, } \\
\text { ultrasonic, dye } \\
\text { penetrant }\end{array}$ & $\begin{array}{l}\text { Remove crack indications and weld } \\
\text { repair }\end{array}$ & 5000287 & LER 01-001-00 & ML011140213 \\
\hline 176 & Exchanger & $\begin{array}{l}\text { Steam generator - } \\
\text { tubing }\end{array}$ & $\begin{array}{l}\text { Inspection at } \\
\text { refueling outage }\end{array}$ & $\begin{array}{l}\text { Eddy current and } \\
\text { point testing }\end{array}$ & Plugged & 5000445 & LER 01-004-00 & ML011100238 \\
\hline 177 & Piping system & Piping & Leaking & NA & Repaired & 5000416 & LER 01-001-00 & ML011140218 \\
\hline 178 & Structural steel & Screw & $\begin{array}{l}\text { Difficulty in full } \\
\text { gripper } \\
\text { engagement }\end{array}$ & $\begin{array}{l}\text { Ultrasonic, visual, } \\
\text { and spring scale } \\
\text { inspection }\end{array}$ & Changed nozzles & 5000395 & LER 99-004-02 & ML011090006 \\
\hline 179 & Exchanger & Inlet-sie tubesheet & $\begin{array}{l}\text { Low condenser } \\
\text { vacuum }\end{array}$ & NA & Removed debris & 5000315 & $\begin{array}{l}\text { LER 001-001- } \\
00\end{array}$ & ML011020251 \\
\hline 180 & Exchanger & $\begin{array}{l}\text { Steam generator } \\
\text { tubing }\end{array}$ & $\begin{array}{l}\text { Primary to } \\
\text { secondary leak }\end{array}$ & $\begin{array}{lr}\text { Eddy } & \text { current } \\
\text { inspection } & \end{array}$ & Plugged or repaired & 5000247 & LER 00-003-01 & ML010890367 \\
\hline 181 & Piping system & Nozzle-to-pipe weld & Leaking & $\begin{array}{l}\text { Dye } \\
\text { penetrant/Ultrasoni } \\
\text { C }\end{array}$ & Weld repair & 5000395 & LER 00-008-01 & ML010790459 \\
\hline 182 & Structural steel & Pump hold-down beam & Jet pump failure & NA & Replaced & 5000254 & LER 02-001-00 & ML020850677 \\
\hline 183 & RPV & $\begin{array}{l}\text { CRDM - control rod } \\
\text { drive } \\
\text { (nozzle) }\end{array}$ & $\begin{array}{l}\text { Boric acid } \\
\text { deposit }\end{array}$ & $\begin{array}{l}\text { Video } \\
\text { inspection/eddy } \\
\text { current/dye } \\
\text { penetrant/ultrasoni } \\
\text { c }\end{array}$ & welded plug & 5000269 & LER 00-006-01 & ML010710015 \\
\hline 184 & Structural steel & Door & Failed to close & NA & Lubricating and cycling & 5000305 & LER 01-001-00 & ML010580336 \\
\hline 185 & Exchanger & $\begin{array}{l}\text { Steam generator } \\
\text { tubing }\end{array}$ & $\begin{array}{l}\text { Primary to } \\
\text { secondary leak }\end{array}$ & NA & NA & 5000247 & LER 00-001-01 & ML010580294 \\
\hline 186 & Piping system & Support & Pipe leaking & NA & Repaired and reinforced & 5000313 & LER 00-001-00 & ML010400162 \\
\hline 187 & Piping system & Piping & Pipe leaking & Visual & Repaired & 5000313 & $\begin{array}{l}\text { LER } \quad 00-001- \\
\text { OOA }\end{array}$ & ML010400162 \\
\hline
\end{tabular}




\begin{tabular}{|c|c|c|c|c|c|c|c|c|}
\hline ID & COMPONENT & SUBCOMPONENT & $\begin{array}{c}\text { HOW } \\
\text { IDENTIFIED }\end{array}$ & $\begin{array}{l}\text { EVALUATION } \\
\text { METHOD }\end{array}$ & REPAIR METHOD & DOCKET & REF & ML NUMBER \\
\hline 188 & Vessel & $\begin{array}{ll}\text { Pressurizer } & \text { heater } \\
\text { sleeves } & \end{array}$ & $\begin{array}{l}\text { Leakage } \\
\text { discovered at } \\
\text { refueling outage }\end{array}$ & NA & Pluuged & 5000382 & LER 00-011-00 & ML003770501 \\
\hline 189 & RPV & CRD - housing & $\begin{array}{l}\text { Inspection at } \\
\text { refueling outage }\end{array}$ & $\begin{array}{l}\text { Visual/dye } \\
\text { penetrant/eddy } \\
\text { current/ultrasonic }\end{array}$ & Repaired & 5000255 & LER 99-004-01 & ML003769646 \\
\hline 190 & Filter & Charcoal & \begin{tabular}{lr}
\multicolumn{2}{l}{ Penetration test } \\
at refueling \\
outage
\end{tabular} & Penetration test & Replaced & 5000251 & LER 00-003-00 & ML003768557 \\
\hline 191 & Exchanger & $\begin{array}{l}\text { Steam generator - } \\
\text { tubing }\end{array}$ & $\begin{array}{l}\text { Eddy current } \\
\text { testing }\end{array}$ & $\begin{array}{l}\text { Eddy } \quad \text { current } \\
\text { testing }\end{array}$ & Plugged & 5000275 & LER 00-010-00 & ML003768576 \\
\hline 192 & Vessel & $\begin{array}{l}\text { Pressurizer heater } \\
\text { sleeves }\end{array}$ & $\begin{array}{l}\text { Inspection at } \\
\text { refueling outage }\end{array}$ & Visual & Cut and weld a plug & 5000529 & LER 00-004-00 & ML003768542 \\
\hline 193 & Piping system & Fitting & Leaking & NA & Replaced & 5000249 & LER 99-003-02 & ML003765638 \\
\hline 194 & Piping system & Piping & $\begin{array}{l}\text { Offgas } \\
\text { condenser low- } \\
\text { level alarm }\end{array}$ & NA & Re-welded & 5000397 & LER 00-007-00 & ML003762455 \\
\hline 195 & Exchanger & ESW room cooler & $\begin{array}{l}\text { Degraded ESW } \\
\text { flow }\end{array}$ & Flow testing & $\begin{array}{l}\text { Clearwell cleaned and chemically } \\
\text { treated }\end{array}$ & 5000483 & LER 00-006-00 & ML003760465 \\
\hline 196 & Structural steel & Weld & NA & NA & Repaired & 5000361 & $\begin{array}{l}\text { LER 949-368- } \\
62\end{array}$ & ML003756122 \\
\hline 197 & Piping system & Fitting & NA & NA & NA & 5000333 & LER 00-010-00 & ML003756877 \\
\hline 198 & Piping system & Piping weld & NA & NA & NA & 5000333 & $\begin{array}{l}\text { LER } \quad 00-010- \\
\text { OOA }\end{array}$ & ML003756877 \\
\hline 199 & Vessel & $\begin{array}{l}\text { Pressurizer heater } \\
\text { sleeves }\end{array}$ & $\begin{array}{l}\text { Inspection } \\
\text { outage }\end{array}$ & $\begin{array}{l}\text { Eddy } \\
\text { current/Ultrasonic }\end{array}$ & Repaired for limited service life & 5000368 & $\begin{array}{l}\text { LER 2000-001- } \\
00\end{array}$ & ML003747697 \\
\hline 200 & Piping system & Piping weld & Test & NA & NA & 5000237 & LER 00-002-00 & ML003738091 \\
\hline 201 & Piping system & $\begin{array}{l}\text { Small bore piping \& } \\
\text { tubing }\end{array}$ & Leaking & NA & Repaired and improved support & 5000261 & $\begin{array}{l}\text { LER 2000-001- } \\
00\end{array}$ & ML003735247 \\
\hline 202 & Exchanger & $\begin{array}{l}\text { Steam generator } \\
\text { tubing }\end{array}$ & $\begin{array}{l}\text { In-service } \\
\text { inspection }\end{array}$ & Eddy current & Plugged or repaired & 5000305 & $\begin{array}{l}\text { LER 920-388- } \\
25\end{array}$ & ML003725181 \\
\hline 203 & Concrete & Walls \& floors & Visual & NA & Repair & 5000316 & LER 00-003-00 & ML051030057 \\
\hline
\end{tabular}




\begin{tabular}{|c|c|c|c|c|c|c|c|c|}
\hline ID & COMPONENT & SUBCOMPONENT & $\begin{array}{c}\text { HOW } \\
\text { IDENTIFIED }\end{array}$ & $\begin{array}{l}\text { EVALUATION } \\
\text { METHOD }\end{array}$ & REPAIR METHOD & DOCKET & REF & ML NUMBER \\
\hline 204 & Piping system & Piping & Pipe rupture & $\mathrm{NA}$ & $\begin{array}{lrl}\text { Replaced } & \text { with } & \text { Chromium- } \\
\text { Molybdenum piping } & \end{array}$ & 5000483 & $\begin{array}{l}\text { LER 1999-003- } \\
01\end{array}$ & ML003712775 \\
\hline 205 & Exchanger & $\begin{array}{l}\begin{array}{l}\text { Steam } \\
\text { tubing }\end{array} \\
\text { generator - }\end{array}$ & Leaking & Eddy current & Plugged or repaired & 5000247 & LER 00-03 & ML003712955 \\
\hline 206 & Piping system & $\begin{array}{l}\text { Small bore piping \& } \\
\text { tubing }\end{array}$ & Chiller tripped & NA & Repaired & 5000390 & LER $81-20$ & ML003708293 \\
\hline 207 & Piping system & $\begin{array}{l}\text { Small bore piping \& } \\
\text { tubing }\end{array}$ & Leaking & NA & Fitting tightened & 5000269 & LER 50-26 & ML003701577 \\
\hline 208 & Exchanger & $\begin{array}{l}\text { Steam generator - } \\
\text { tubing }\end{array}$ & $\begin{array}{l}\text { Inspection at } \\
\text { refueling outage }\end{array}$ & Eddy current & Plugged & 5000250 & $\begin{array}{l}\text { LER 2000-001- } \\
00\end{array}$ & ML003705889 \\
\hline 209 & Piping system & Suction line weld & Leaking & NA & Repaired & 5000382 & LER 00-003-00 & ML003703686 \\
\hline 210 & Exchanger & $\begin{array}{l}\text { Steam generator - } \\
\text { tubing }\end{array}$ & $\begin{array}{l}\text { Primary to } \\
\text { secondary } \\
\text { leaking }\end{array}$ & NA & Plugged & 5000247 & LER 00-001-00 & ML020350573 \\
\hline 211 & Piping system & Elbow & Leaking & $\begin{array}{l}\text { Visual \& laboratory } \\
\text { examination }\end{array}$ & Elbow replaced & 5000269 & $\begin{array}{l}\text { LER 2000-001- } \\
00\end{array}$ & ML003694353 \\
\hline 212 & Piping system & Weld & Leaking & $\begin{array}{l}\text { NDE \& Laboratory } \\
\text { analysis }\end{array}$ & Weld pad buildup and fillet weld & 5000313 & $\begin{array}{l}\text { LER 2000-003- } \\
00\end{array}$ & ML003693846 \\
\hline 213 & Containment & Liner & Inspection & $\begin{array}{l}\text { Visual \& magnetic } \\
\text { particle exam }\end{array}$ & Replacement of degraded seal & 5000315 & LER 98-011-03 & ML003695085 \\
\hline 214 & Filter & $\begin{array}{l}\text { Nuts holding in } \\
\text { Charcoal tray }\end{array}$ & Surveillance test & Visual & Tightened & 5000317 & $\begin{array}{l}\text { LER 410-495- } \\
20\end{array}$ & ML003687837 \\
\hline 215 & Containment & Liner & Inspection & NA & Repair & 5000315 & LER 00-001-00 & ML003687066 \\
\hline 216 & Filter & Charcoal & $\begin{array}{l}\text { Sample analysis } \\
\text { per surveillance } \\
\text { requirement }\end{array}$ & $\begin{array}{l}\text { Laboratory } \\
\text { analysis }\end{array}$ & Replacement & 5000498 & LER 99-007-01 & ML003674168 \\
\hline 217 & Exchanger & $\begin{array}{l}\text { Steam generator - } \\
\text { tubing }\end{array}$ & $\begin{array}{l}\text { Eddy current } \\
\text { inspection }\end{array}$ & $\begin{array}{l}\text { Eddy current } \\
\text { inspection }\end{array}$ & Plugged & 5000364 & LER 99-002-00 & ML993480199 \\
\hline 218 & RPV & Nozzle-safe end weld & $\begin{array}{l}\text { Ultrasonic } \\
\text { examination at } \\
\text { refuelling outage }\end{array}$ & Ultrasonic & NA & 5000331 & LER 99-00 & ML993500158 \\
\hline 219 & Piping system & $\begin{array}{l}\text { Small bore piping \& } \\
\text { tubing }\end{array}$ & $\begin{array}{l}\text { Excessive water } \\
\text { inventory in } \\
\text { ESSMSRD tank }\end{array}$ & NA & Repaired & 5000333 & LER 99-012-00 & ML993470148 \\
\hline
\end{tabular}




\begin{tabular}{|c|c|c|c|c|c|c|c|c|}
\hline ID & COMPONENT & SUBCOMPONENT & $\begin{array}{c}\text { HOW } \\
\text { IDENTIFIED } \\
\end{array}$ & $\begin{array}{l}\text { EVALUATION } \\
\text { METHOD }\end{array}$ & REPAIR METHOD & DOCKET & REF & ML NUMBER \\
\hline 220 & Piping system & Piping weld & Leaking & Dye penetrant & Ground out and re-welded & 5000266 & $\begin{array}{l}\text { LER 1999-012- } \\
00\end{array}$ & ML993420164 \\
\hline 221 & RPV & CRDM Seal housing & $\begin{array}{l}\text { Inspection at } \\
\text { refueling outage }\end{array}$ & $\begin{array}{lrr}\text { Visual \& } & \text { dye } \\
\text { penetrant \& } & \text { eddy } \\
\text { current exam } & \end{array}$ & Repaired & 5000255 & LER 99-00 & ML993420206 \\
\hline 222 & Exchanger & $\begin{array}{l}\text { Steam generator - } \\
\text { tubing }\end{array}$ & Inspection & Eddy current & plugged & 5000499 & LER 99-007-00 & ML993140290 \\
\hline 223 & Filter & Charcoal & Surveillance test & Tested by vendor & Replaced & 5000333 & LER 99-009-00 & ML993250125 \\
\hline 224 & Piping system & Nozzle & $\begin{array}{l}\text { Walkdown at } \\
\text { refueling outage }\end{array}$ & Visual & $\begin{array}{l}\text { Cut off the existing nozzle and } \\
\text { installed new nozzle }\end{array}$ & 5000528 & LER 99-006-00 & ML993140352 \\
\hline 225 & Filter & Damper & $\begin{array}{l}\text { Negative } \\
\text { pressure in CR }\end{array}$ & Visual & $\begin{array}{l}\text { Temporary mechanical modification } \\
\text { to secure the manual balancing } \\
\text { damper }\end{array}$ & 5000289 & LER 99-003-01 & ML993140218 \\
\hline 226 & Exchanger & $\begin{array}{l}\text { Steam generator } \\
\text { tubing }\end{array}$ & $\begin{array}{l}\text { Eddy current } \\
\text { testing }\end{array}$ & $\begin{array}{l}\text { Eddy } \\
\text { testing }\end{array}$ & Plugged & 5000445 & LER 98-006-00 & ML993080064 \\
\hline
\end{tabular}


Table 3-4 Abbreviation for Aging Mechanisms

\begin{tabular}{|l|l|}
\hline ABBREVIATION & DEFINITION \\
\hline IGSCC & Intergranular stress corrosion cracking \\
\hline IDSCC & Interdendritic SCC \\
\hline HSC & Hydrogen stress corrosion \\
\hline TGSCC & Transgranular SCC \\
\hline SCC & Stress corrosion cracking \\
\hline PWSCC & Primary water SCC \\
\hline IGA & Intergranular attack \\
\hline ODSCC & Outer diameter SCC \\
\hline ODIGA & Outer diameter IGA \\
\hline FAC & Flow accelerated corrosion \\
\hline
\end{tabular}


Table 3-5 Number of DORs and NPP Units by PAAE for LER 1999-2007

\begin{tabular}{cccc}
\hline \multicolumn{4}{c}{ LER 1999-2007 } \\
\hline PAAE & DORs & NPP Units & DORs/NPP Units \\
\hline 23 & 2 & 2 & 1.000 \\
24 & 4 & 4 & 1.000 \\
25 & 3 & 3 & 1.000 \\
26 & 11 & 9 & 1.222 \\
27 & 10 & 9 & 1.111 \\
28 & 11 & 10 & 1.100 \\
29 & 10 & 8 & 1.250 \\
30 & 11 & 7 & 1.571 \\
31 & 21 & 15 & 1.400 \\
32 & 19 & 14 & 1.357 \\
33 & 30 & 20 & 1.500 \\
34 & 22 & 12 & 1.833 \\
35 & 18 & 15 & 1.200 \\
36 & 16 & 14 & 1.143 \\
37 & 10 & 10 & 1.000 \\
38 & 7 & 5 & 1.400 \\
39 & 6 & 5 & 1.200 \\
40 & 7 & 5 & 1.400 \\
41 & 5 & 4 & 1.250 \\
\hline
\end{tabular}

Table 3-6 Number of DORs and NPP Units by PAAE for LER 1985-1996

\begin{tabular}{cccc}
\hline \multicolumn{4}{c}{ LER 1985-1996 } \\
\hline PAAE & DORs & NPP Units & DORs/NPP Units \\
\hline \hline 8 & 1 & 1 & 1.00 \\
12 & 3 & 3 & 1.00 \\
13 & 2 & 2 & 1.00 \\
14 & 5 & 4 & 1.25 \\
15 & 4 & 3 & 1.33 \\
16 & 11 & 10 & 1.10 \\
17 & 11 & 10 & 1.10 \\
18 & 12 & 9 & 1.33 \\
19 & 9 & 8 & 1.13 \\
20 & 11 & 11 & 1.00 \\
21 & 11 & 10 & 1.10 \\
22 & 13 & 10 & 1.30 \\
23 & 8 & 8 & 1.00 \\
24 & 22 & 15 & 1.47 \\
25 & 15 & 11 & 1.36 \\
26 & 10 & 9 & 1.11 \\
27 & 8 & 7 & 1.14 \\
28 & 6 & 6 & 1.00 \\
29 & 8 & 6 & 1.33 \\
30 & 1 & 1 & 1.00 \\
\hline
\end{tabular}




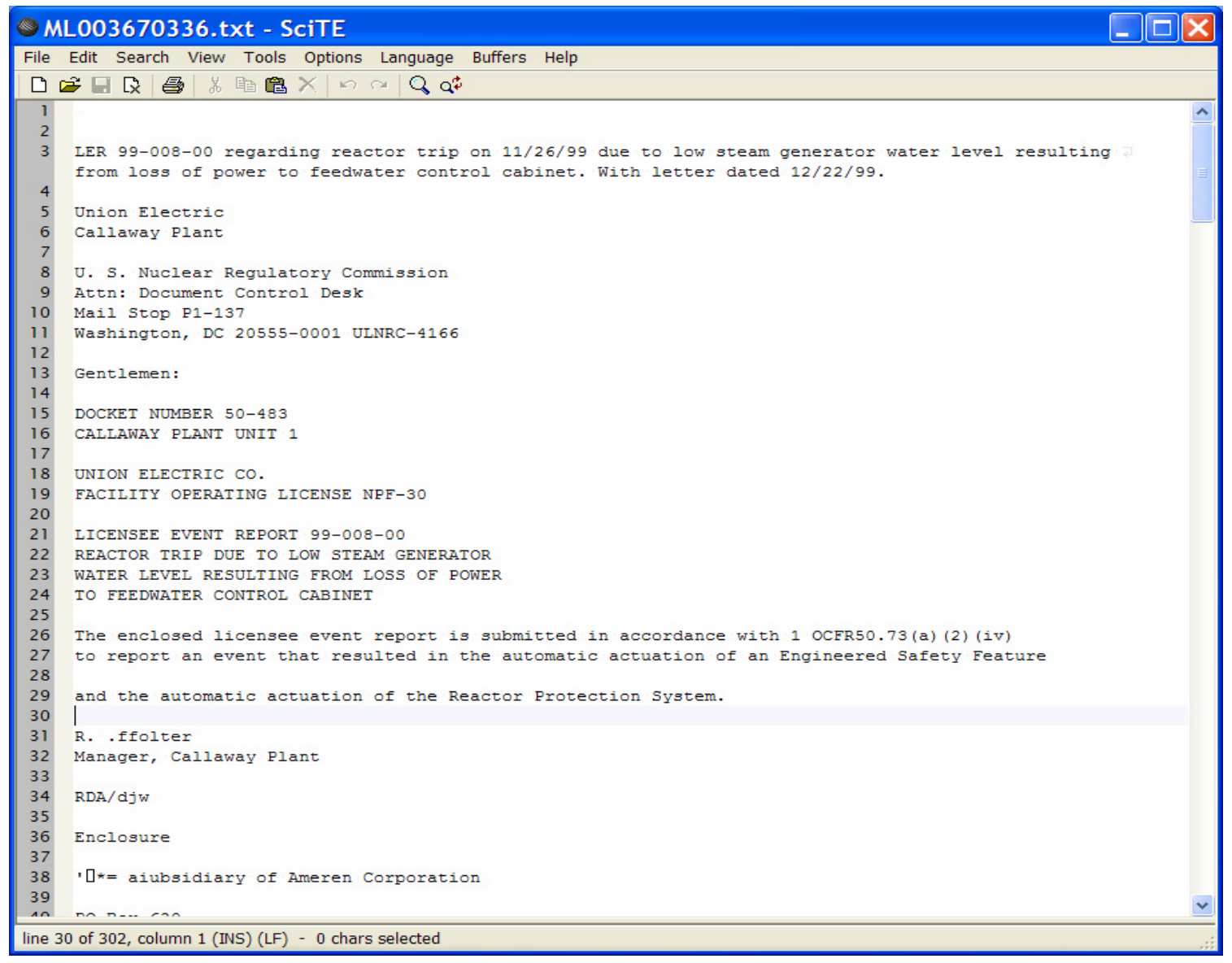

Figure 3-1 A Sample LER in Text Format (Beginning Part) 


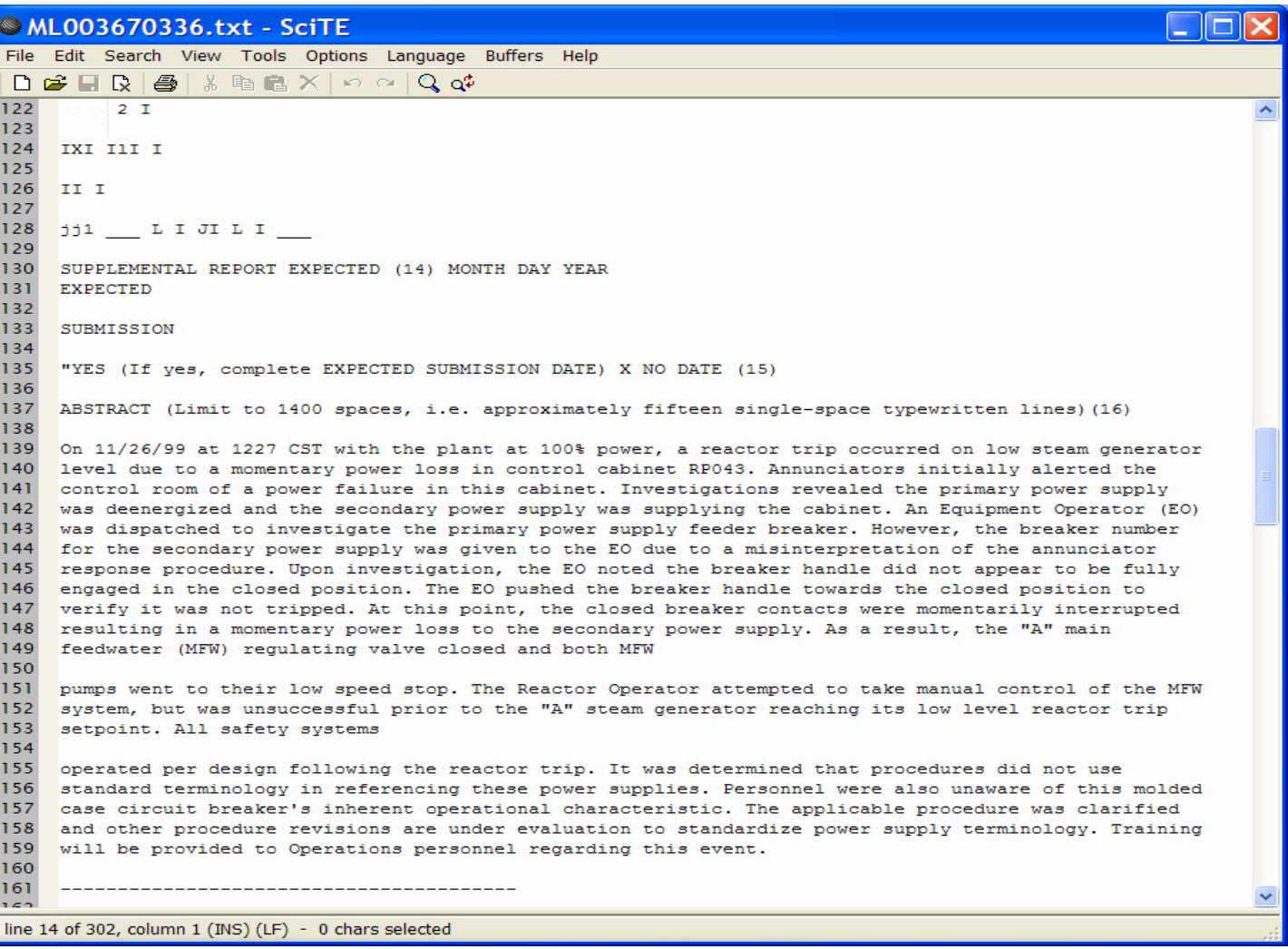

Figure 3-2 A Sample LER in Text Format (Abstract Part)

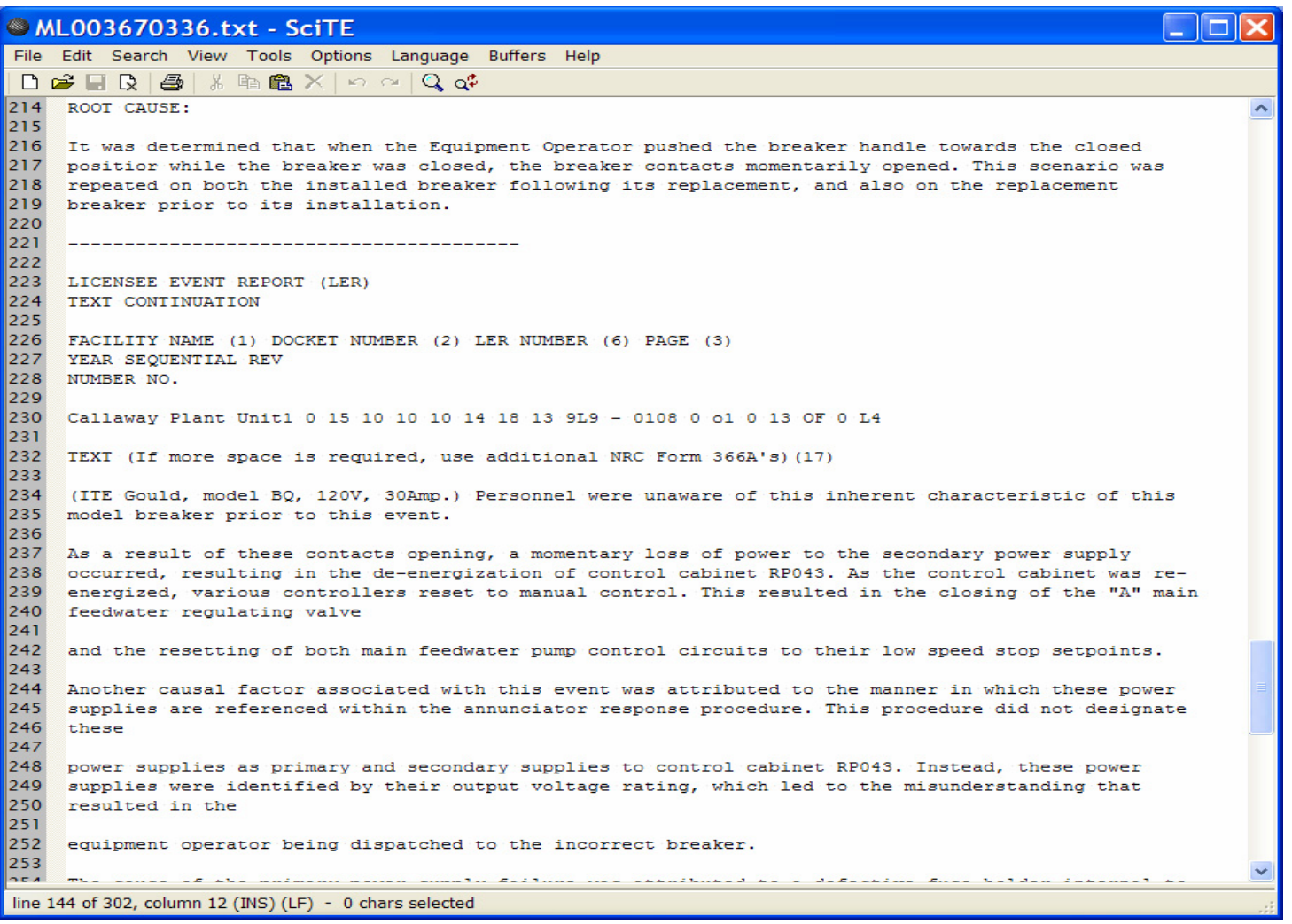

Figure 3-3 A Sample LER in Text Format (Cause Part) 


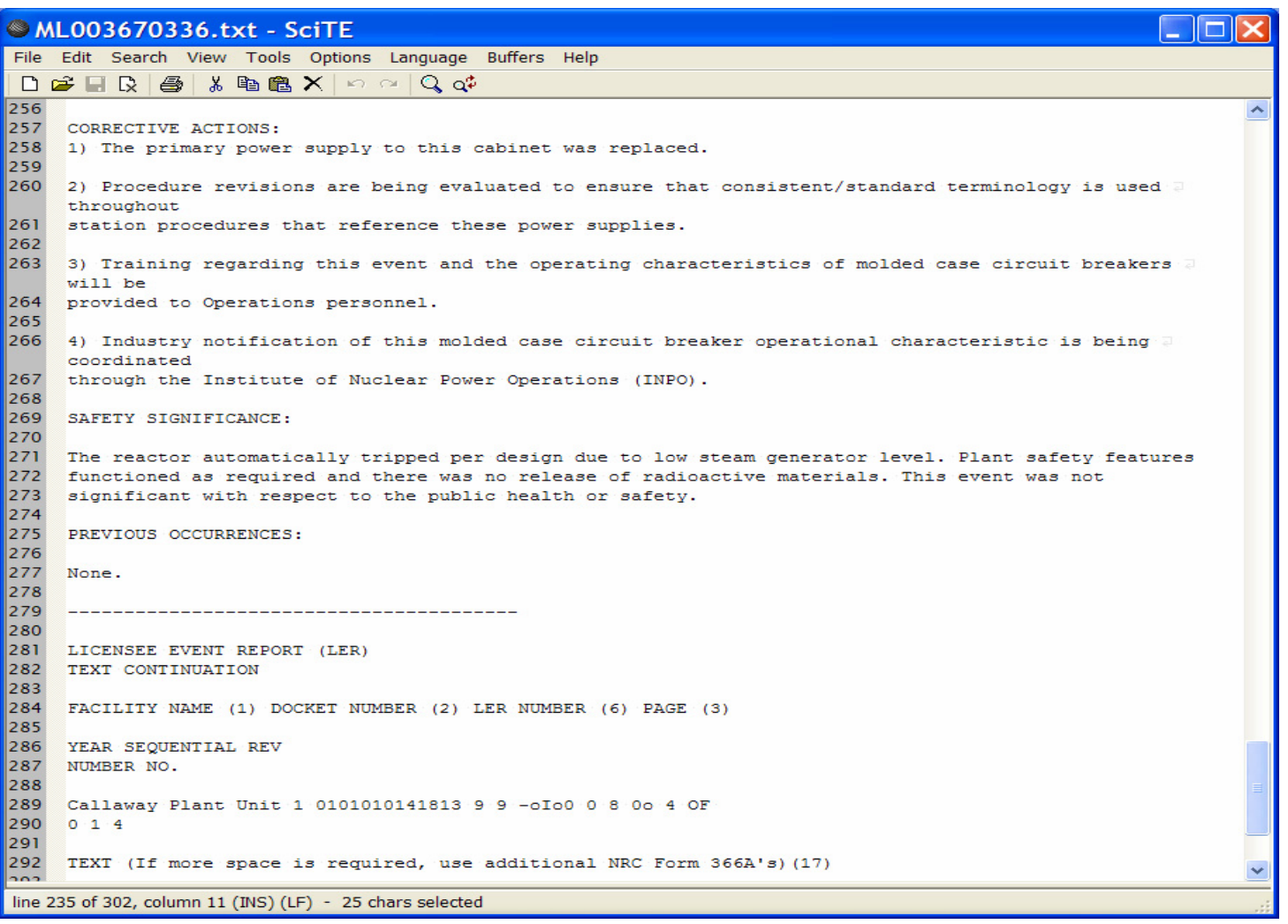

Figure 3-4 A Sample LER in Text Format (Action Part) 


\section{LICENSEE EVENT REPORT (LER)}

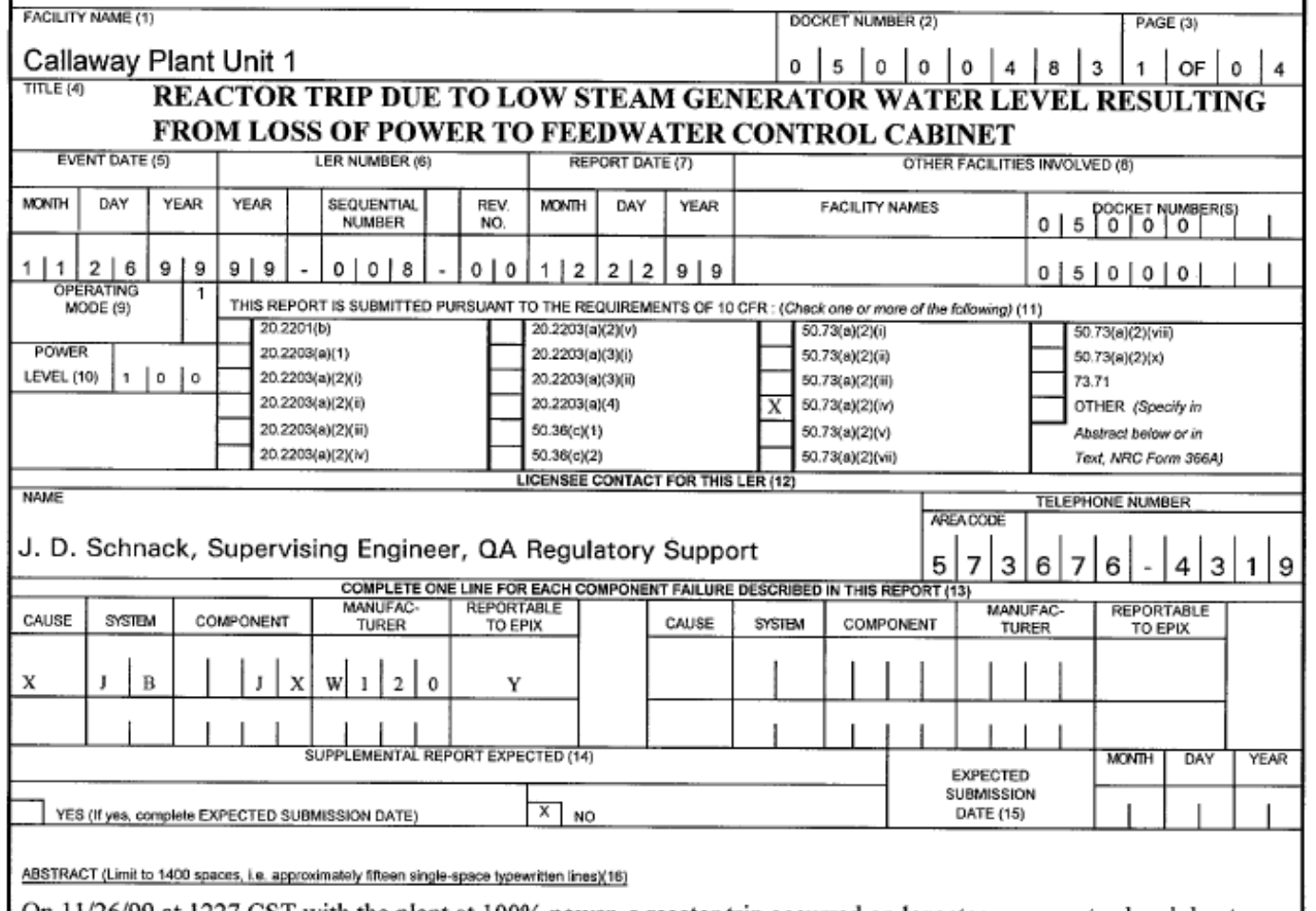

On $11 / 26 / 99$ at $1227 \mathrm{CST}$ with the plant at $100 \%$ power, a reactor trip occurred on low steam generator level due to a momentary power loss in control cabinet RP043. Annunciators initially alerted the control room of a power failure in this cabinet. Investigations revealed the primary power supply was deenergized and the secondary power supply was supplying the cabinet. An Equipment Operator (EO) was dispatched to investigate the primary power supply feeder breaker. However, the breaker number for the secondary power supply was given to the EO due to a misinterpretation of the annunciator response procedure. Upon investigation, the EO noted the breaker handle did not appear to be fully engaged in the closed position. The EO pushed the breaker handle towards the closed position to verify it was not tripped. At this point, the closed breaker contacts were momentarily interrupted resulting in a momentary power loss to the secondary power supply. As a result, the "A" main feedwater (MFW) regulating valve closed and both MFW pumps went to their low speed stop. The Reactor Operator attempted to take manual control of the MFW system, but was unsuccessful prior to the "A" steam generator reaching its low level reactor trip setpoint. All safety systems operated per design following the reactor trip. It was determined that procedures did not use standard terminology in referencing these power supplies. Personnel were also unaware of this molded case circuit breaker's inherent operational characteristic. The applicable procedure was clarified and other procedure revisions are under evaluation to standardize power supply terminology. Training will be provided to Operations personnel regarding this event.

Figure 3-5 A Sample LER in PDF Format (Abstract Part) 


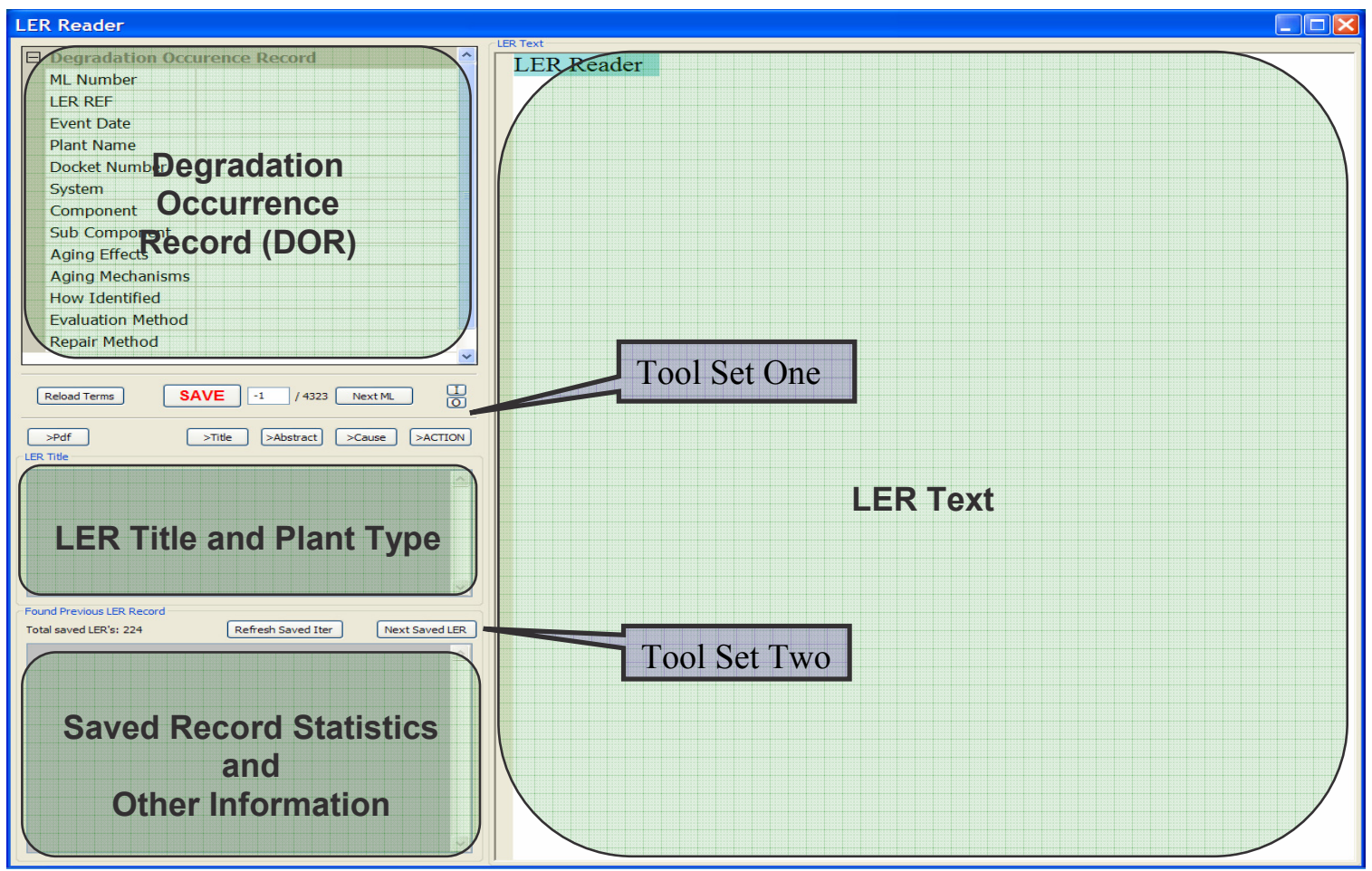

Figure 3-6 The Annotated LER Reader Graphical User Interface

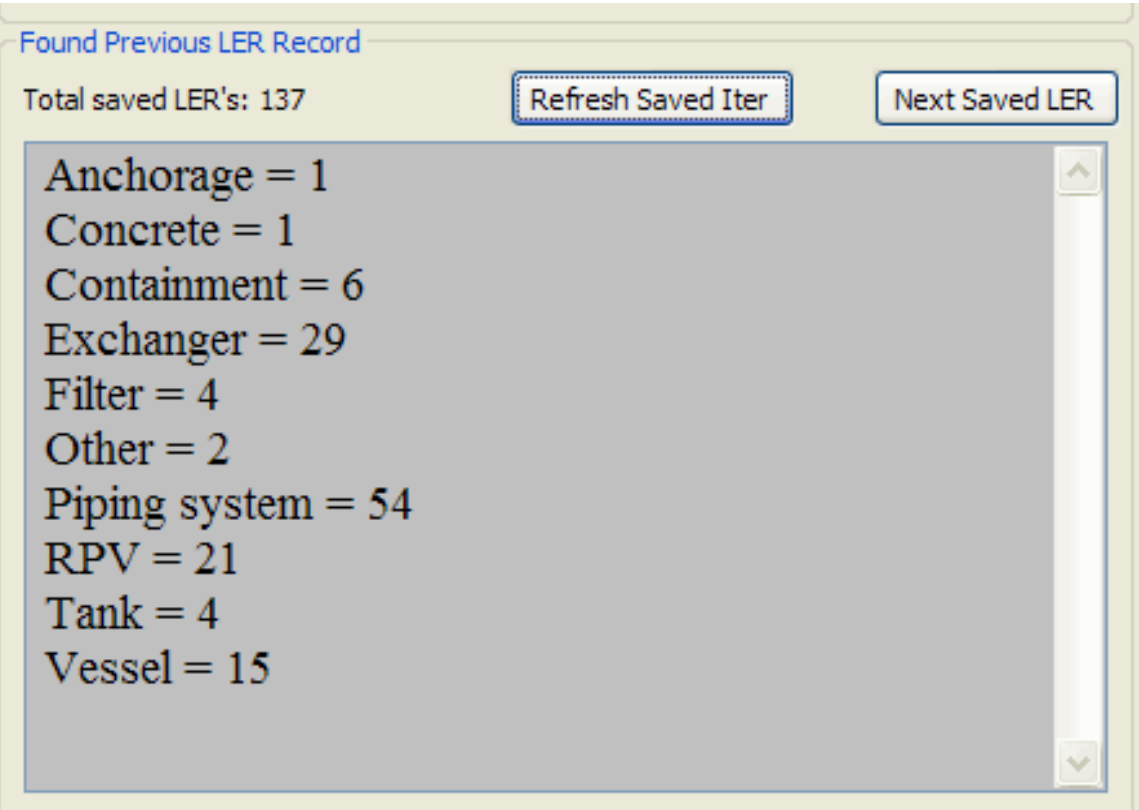

Figure 3-7 DOR Statistics in the LER Reader 


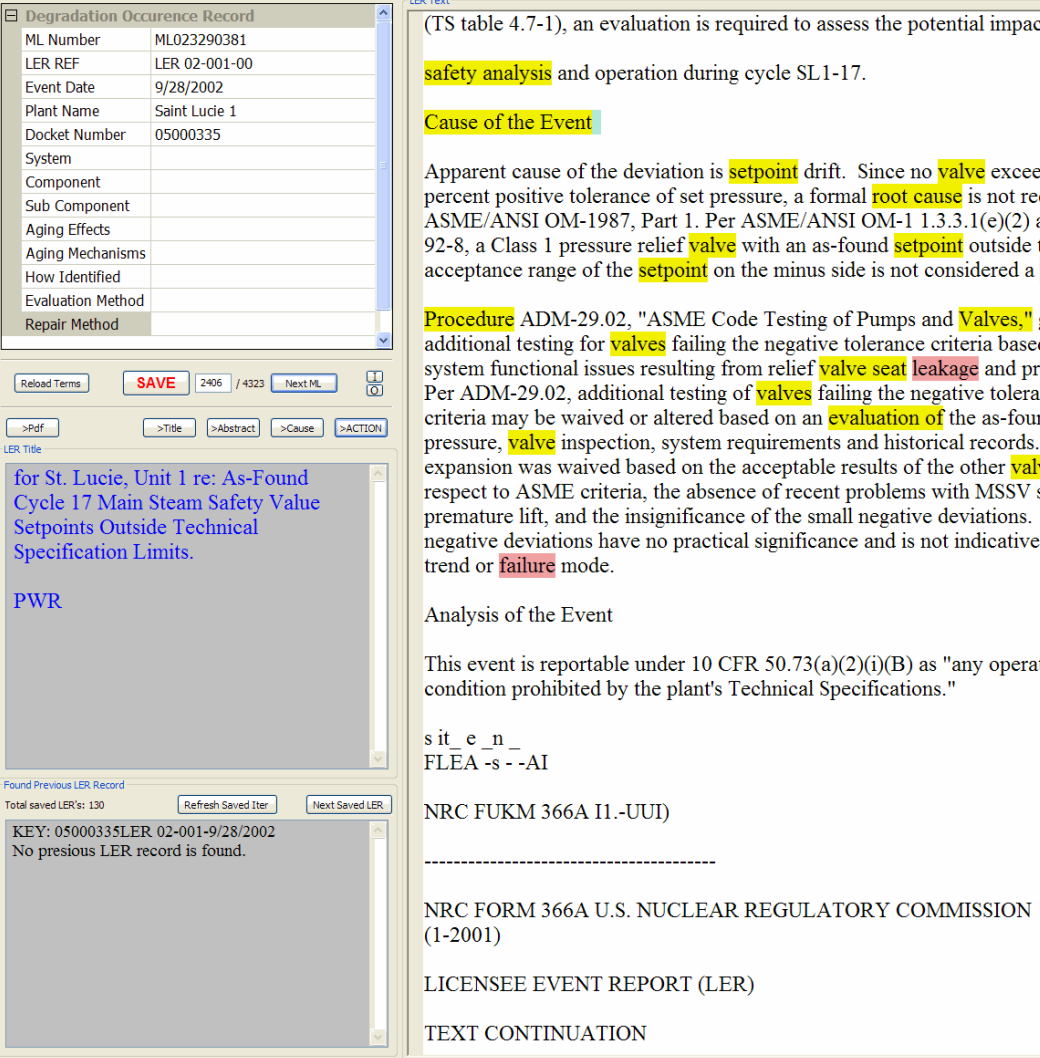

Figure 3-8 An LER Related to Valve 


\begin{tabular}{|c|c|}
\hline \multicolumn{2}{|c|}{9 Degradation Occurence Record } \\
\hline ML Number & ML023180480 \\
\hline LER REF & LER 02-001-00 \\
\hline Event Date & $09 / 14 / 2002$ \\
\hline Plant Name & North Anna 2 \\
\hline Docket Number & 05000339 \\
\hline System & RCS - reactor coolant system \\
\hline Component & RPV \\
\hline Sub Component & Penetration \\
\hline Aging Effects & Gracking \\
\hline Aging Mechanisms & s PWSCC with initial hot-short crack \\
\hline How Identified & Inspection at refueling outage \\
\hline Evaluation Method & Visual + NDE \\
\hline Repair Method & Replacement of RPV head \\
\hline Reload Terms & $\begin{array}{llll} & 2405 & 4323 & \text { NextML } \\
\end{array}$ \\
\hline 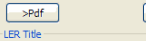 & 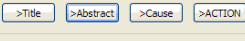 \\
\hline
\end{tabular}

(4)

Reactor Vessel Head Leakage due to Hot Short Cracking and Primary Water

Stress Corrosion Cracking

PWR

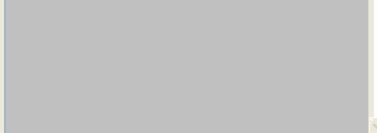

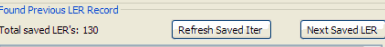

SAVED -

ML023180480 | LER 02-001-00 | 09/14/2002

Noth Anna $2|05000339|$ RCS - reactor coolant
system | RPV | Penetration | Cracking | PWSCC

with initial hot-short cracking $\mid$ Inspection at

refueling outage $\mid$ Visual + NDE | Replacement of

RPV head
TO EP.XTO EI

B $\mid$ AB RPV R380 Yes

SUPPLEMENTAL REPORT EXPECTED (14) EXPECTED MONTH DAY YEAR

SUBMISSION

YES(Iyes, complete EXPECTED SUBMISSION DATE) X I NO DATE (15) 111

ABSTRACT (Limit to 1400 spaces, i.e., approximately 15 single-spaced typewritten lines) (16)

On September 8, 2002, North Anna Unit 2 was shutdown for a scheduled refueling outage. A qualified, visual barehead inspection of the reactor vessel head and penetrations was performed to identify evidence of leakage as required by NRC Bulletin 2001-01. On September 14, 2002, with Unit 2 in Mode 6 , the qualified, visual barehead inspection on penetrations 21 and 31 identified through-wall leakage based on the presence of boric acid deposited on the reactor head at these penetrations. A nonemergency 8-hour notification was made to the NRC, at 2214 hours, on September 14, 2002, in

accordance with 10CFR50.72(b)(3)(ii)(A). The event is also reportable in accordance with

10CFR50.73(a)(2)(i)(B). The apparent cause of the event was hot-short cracking, which occurred

during original fabrication of the reactor vessel head, that was accelerated by primary water stress corrosion cracking. Corrective action is to replace the reactor head. No significant safety

consequences resulted from this event since RCS unidentified leakage was well below Technica Specification limits. No safety concerns exist since cracking that is contained entirely in the weld metal

would not lead to nozzle ejection. The health and safety of the public were not affected at any time during this event.

NRC FORM 366 (1-2001)

NRC FORM 366A U.S. NUCLEAR REGULATORY COMMISSION (7-2001)

LICENSEE EVENT REPORT (LER)

TEXT CONTINUATION

FACILITY NAME (1) DOCKET LER NUMBER (6) PAGE (3)

I YEAR I SEQUENTIAL I REVISION

1| NUMBER | NUMBER

NORTH ANNA POWER STATION 05000 - 3392002 --001 -- 002 of 11

Figure 3-9 An LER and its DOR (Saved) 


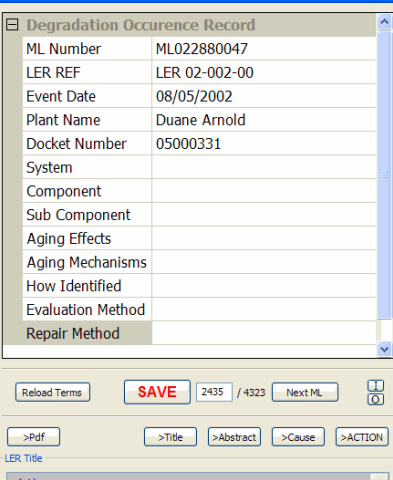

(4)

Technical Specification Required

Shutdown Due to Residual Heat

Removal Service Water (RHRSW)

Strainer

BWR

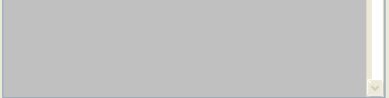

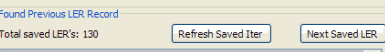

KEY: 05000331LER 02-002-08/05/2002

KEY: $05000331 L E R$ 02-002-08/05
No presious LER record is found.

The plant commenced startup on August 11, 2002.

\section{Cause of Event:}

The introduction and accumulation of large amounts of algae from the Cedar River is the cause of the RHRSW strainer high differentiall pressures that resulted in the plant shutdown. Underlying causes include less than adequate river monitoring and management, changes in the Cedar River conditions, and a failure of corrective actions from a previous similar event.

NRC FORM 366A (1-2001)

NRC FORM 366A U.S. NUCLEAR REGULATORY COMMISSION

$(1-2001)$

LICENSEE EVENT REPORT (LER)

TEXT CONTINUATION

FACILITY NAME (1) DOCKET NUMBER (2) LER NUMBER (6) PAGE 3

Y 'SEQUENTIAL REVISION

Duane Arnold Energy Center 05000331 YEAR NUMBER NUMBER

f $2002--002-003$ of 7

TEXT (Ifimore space is required, use additional copies of NRC Form 366A) (17)

II. Cause of Event (continued):

River Monitoring and Management:

Internal and external operating experience reviews indicate previous site difficulties and less than adequate river maintenance and monitoring to prevent formation of conditions that promote algae growth. Development of high levels of silt build-up (discovered in July, 2002) in the river upstream and directly in front of the intake structure allowed higher than normal silt build-up internal to the intake pits and stilling basin. Evaluations of the silt by external consultants show significant levels of nutrients, which can support algae growth.

Changes in the Cedar River Conditions:

Figure 3-10 An LER Required Careful Review 


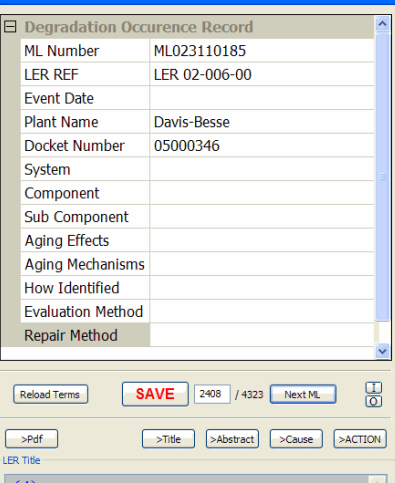

(4).

Emergency Diesel Generator Exhaust Piping Not Adequately Protected From Potential Tornado-Generated Missiles

PWR
Technical Specifications, these conditions represent conditions prohibited by

the Technical Specifications, and are therefore reportable in accordance with

10CFR50.73(a) (2) (i) (B).

\section{APPARENT CAUSE OF OCCURRENCE:}

The unprotected EDG Exhaust Piping and unprotected MSSVs have been in this condition since initial operation of the DBNPS. The preliminary apparent cause for these conditions is that the original design for the associated tornado missile barriers was inadequate, based on the DBNPS USAR description of tornado missile protection.

Evaluations continue with respect to the apparent cause(s) of these occurrences. Additional information that may be developed pertinent to the apparent cause of these conditions will be provided in a supplement to this report within 30 days following restart of DBNPS.

ANALYSIS OF OCCURRENCE:

The Electric Power Research Institute (EPRI) tornado missile methodology contained in EPRI Report NP-2005, "Tornado Missile Risk Evaluation Methodology, dated August 1981 was used to determine the probability of a tornado missile strike for the unprotected portions of the systems required in the event of a tornado. This EPRI methodology is implemented using the computer program TORMIS, which develops the probability of tornado missiles striking the modeled plant structures and other targets using Monte Carlo probability techniques. EPRI Report NP-2005 has been evaluated generically by the NRC in a Safety Evaluation Report dated October 26, 1983, which concluded that TORMIS is an acceptable approach for demonstrating compliance with the requirements of 10 CFR 50 Appendix A General Design Criteria 2 regarding protection of safetyrelated plant features from the effects of tornado and high wind generated

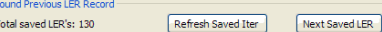

KEY: 05000346LER 02-006-

NRC FORM 366A (1.2001)

No presious

NRC FORM 366A U.S. NUCLEAR REGULATORY COMMISSION

$(1-2001)$

LICENSEE EVENT REPORT (LER)

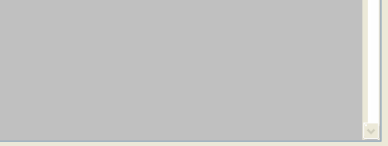

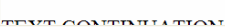

Figure 3-11 An LER Possibly Related to a Design Problem (Required Careful Review) 


\begin{tabular}{|c|c|c|}
\hline \multicolumn{3}{|c|}{$\square$ Degradation Occurence Record } \\
\hline ML Number & ML023240226 & \\
\hline LER REF & LER 02-002-00 & \\
\hline \multicolumn{3}{|l|}{ Event Date } \\
\hline Plant Name & Pilgrim 1 & \\
\hline Docket Number & 05000293 & \\
\hline \multicolumn{3}{|l|}{ System } \\
\hline \multicolumn{3}{|l|}{ Component } \\
\hline \multicolumn{3}{|l|}{ Sub Component } \\
\hline \multicolumn{3}{|l|}{ Aging Effects } \\
\hline \multicolumn{3}{|l|}{ Aging Mechanisms } \\
\hline \multicolumn{3}{|l|}{ How Identified } \\
\hline \multicolumn{3}{|l|}{ Evaluation Method } \\
\hline \multicolumn{3}{|l|}{ Repair Method } \\
\hline Relood Terms & SAVE $2402 / 4423$ NextML & 10 \\
\hline 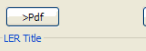 & $\rightarrow$ Tite $>$ abstratt $>$ Cause & \\
\hline
\end{tabular}

\section{(4)}

Control Room High Efficiency Air Filtration System Inoperable Due to Inadequate Post Modification Testing BWR

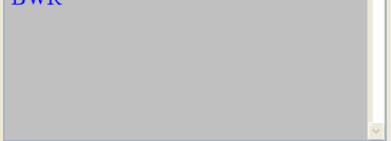

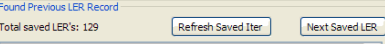

KEY: 05000293LER 02-002-

KEY: 05000293LER 02-002-
No presious LER record is found
This condition occurred at 100 percent reactor power with the reactor mode selector switch in the RUN position

\section{CAUSE}

The root cause of this event was a human performance error by'a utility licensed operator with respect to the inappropriate decision to declare the "B" CRHEAFS train operable without all procedural requirements satisfied following the humidity switch replacement. Contributing to this unintentional human performance error were procedural weaknesses that made it more difficult to determine the correct operability testing requirements. In addition, the design change, under which the humidity switch was replaced, incorrectly wired the switch such that the switch would not energize the heater when relative humidity exceeded $70 \%$.

\section{CORRECTIVE ACTION}

Corrective actions taken include the following:

a) "A" CRHEAFS train was restored to operable status on September 12, 2002 at 09:45.

b) The "B" CRHEAFS train humidity switch was bypassed and "B" CRHEAFS train was declared operable on September 13, 2002 at 23:00

c) An Operations night order describing the event was issued. As an interim measure, the night order also required the applicable procedure to be place kept during Limiting Condition for Operation (LCO) processing and to obtain Operations management concurrence prior to clearing LCOs that involve physical plant work. Subsequently, an Operations standing order was issued on October 8,2002 specifying the same requirements as the night order.

d) All current tracking LCOs were reviewed for potential operability testing that may have been overlooked. No examples were identified.

e) All operations shift crews were briefed on the event and the management expectations for declaring safety related equipment operable was discussed.

NRC FORM 366A (6-1998)

NRC Form 366A ', , , . \{ai j U.S. NUCLEAR REGULATORY COMMISSION

Figure 3-12 An LER Related to Human Error and Electric Parts 


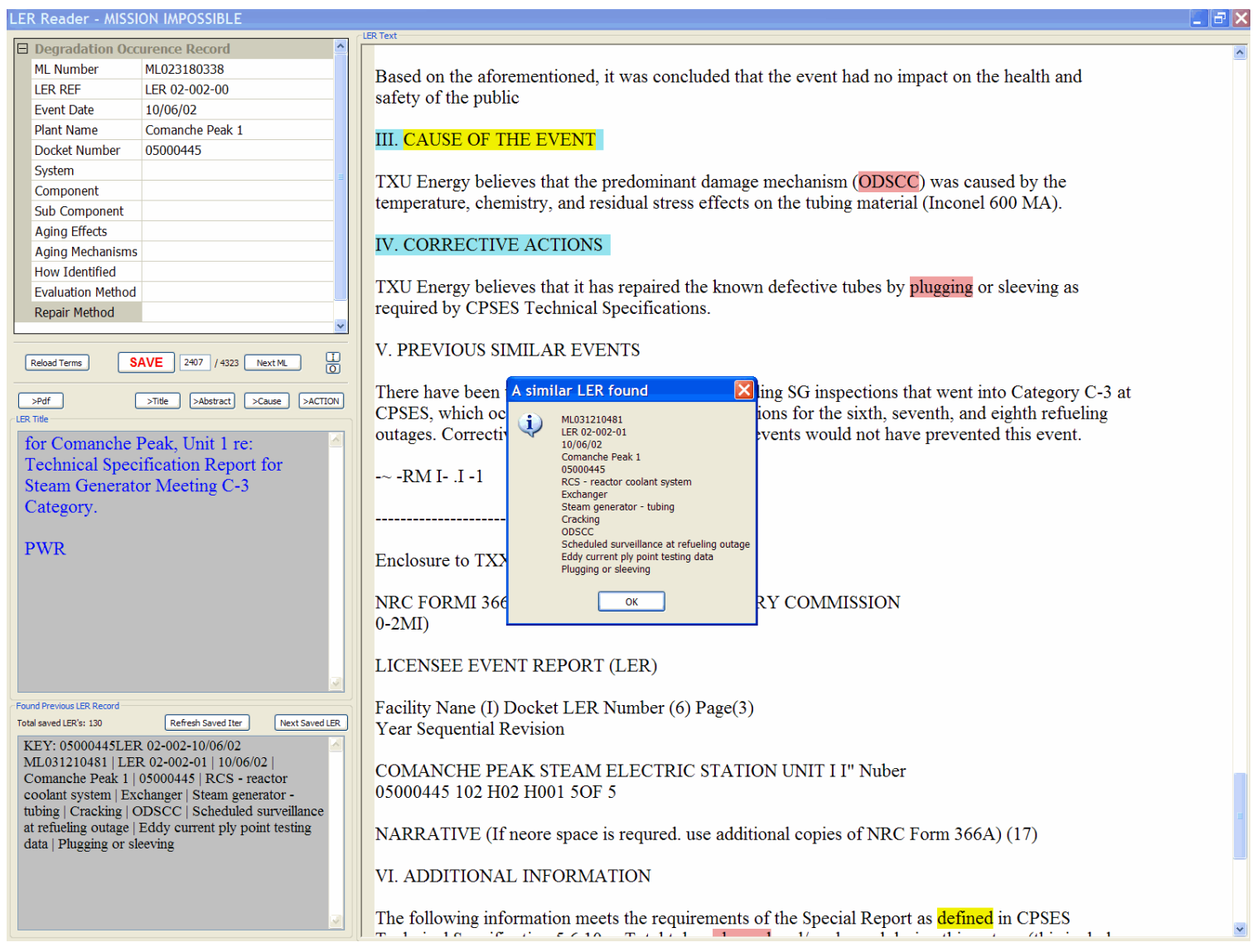

Figure 3-13 An LER with an Existing DOR 


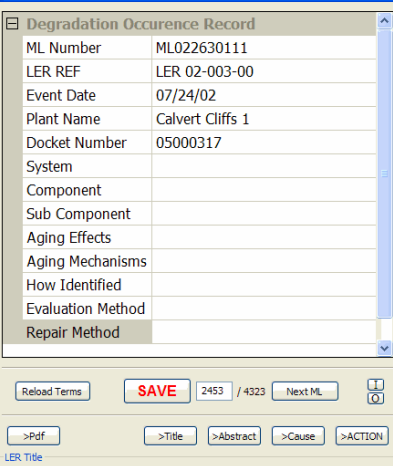

Reactor Trip Due to Loss of Reactor

Coolant Pump Motor Oil

PWR

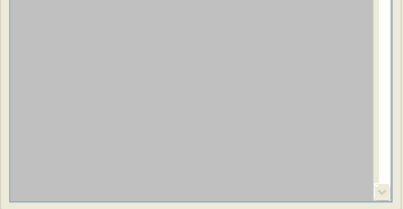

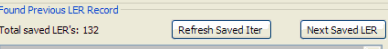

KEY: 05000317LER 02-003-07/24/02

ML022630111 | LER 02-003-00 | 07/24/02 | Calvert

Piping system | Piping weld | Cracking | F t pump

Low level alarm $\mid$ NA $\mid$ NA
No structures, systems, or components were inoperable at the start of the event that would have

contributed to the event. This event is only applicable to Unit 1 because the failure was identified

as a weld failure specific to an oil return line on $11 \mathrm{~A} \mathrm{RCP}$ motor.

\section{CAUSE OF EVENT}

The immediate physical cause of this event was identified as the failure of a butt weld on the oil return line from the oil cooler on I 1A RCP motor. The weld failure resulted in a through-wall crack that extended approximately one-third of the circumference of the pipe. This crack allowed the lubricating oil to drain from the reservoir, which resulted in oil starvation of the thrust bearing

and subsequent overheating. The immediate physical cause of the butt weld failure was a high cycle fatigue failure resulting from the lack of full penetration in the weld.

An additional cause identified during the root cause investigation was the failure to identify and correct the weld deficiency prior to this event. A Unit 2 forced outage on October 25, 2001 was the result of a similar butt weld failure on component cooling water piping to the $22 \mathrm{~A} \mathrm{RCP}$ motor upper bearing oil reservoir. One of the corrective actions from the Unit 2 forced outage was to upper bearing oil reservoir. One of the corrective actions from the Unit 2 forced outage was to
inspect all other similar butt welds in Unit 1 RCPs, and to replace any welds identified without full inspect all other similar butt welds in Unit 1 RCPs, and to replace any welds identified without full
penetration welds. The failed butt weld on the 11A RCP motor oil cooler return line that caused penetration welds. The failed butt weld on the 11A RCP motor oil cooler return line that caused
the subject event was in the population of welds requiring inspection, however, the weld was not inspected. An underlying cause for the trip was a failure by station personnel to use a systematic method to identify and preemptively repair all of the affected Unit 1 RCP motor butt welds.

Ill. ANALYSIS OF EVENT

This event is reportable because of the resulting manual actuation of a valid reactor trip, in accordance with 10 CFR 50.73(a)(2)(iv)(a). No actual safety consequences resulted from this event because all required safety systems were available and functioned as designed, with the

NRC FORM 366AU.S. NUCLEAR REGULATORY COMMISSION

(1-2001)

LICENSEE EVENT REPORT (LER)

1. FACILITY NAME 2. DOCKET 6. LER NUMBER 3. PAGE

I SEQUENTIAL IREVISION

YEAR | NUMBER INUMBER

Figure 3-14 An LER with Two DORs 


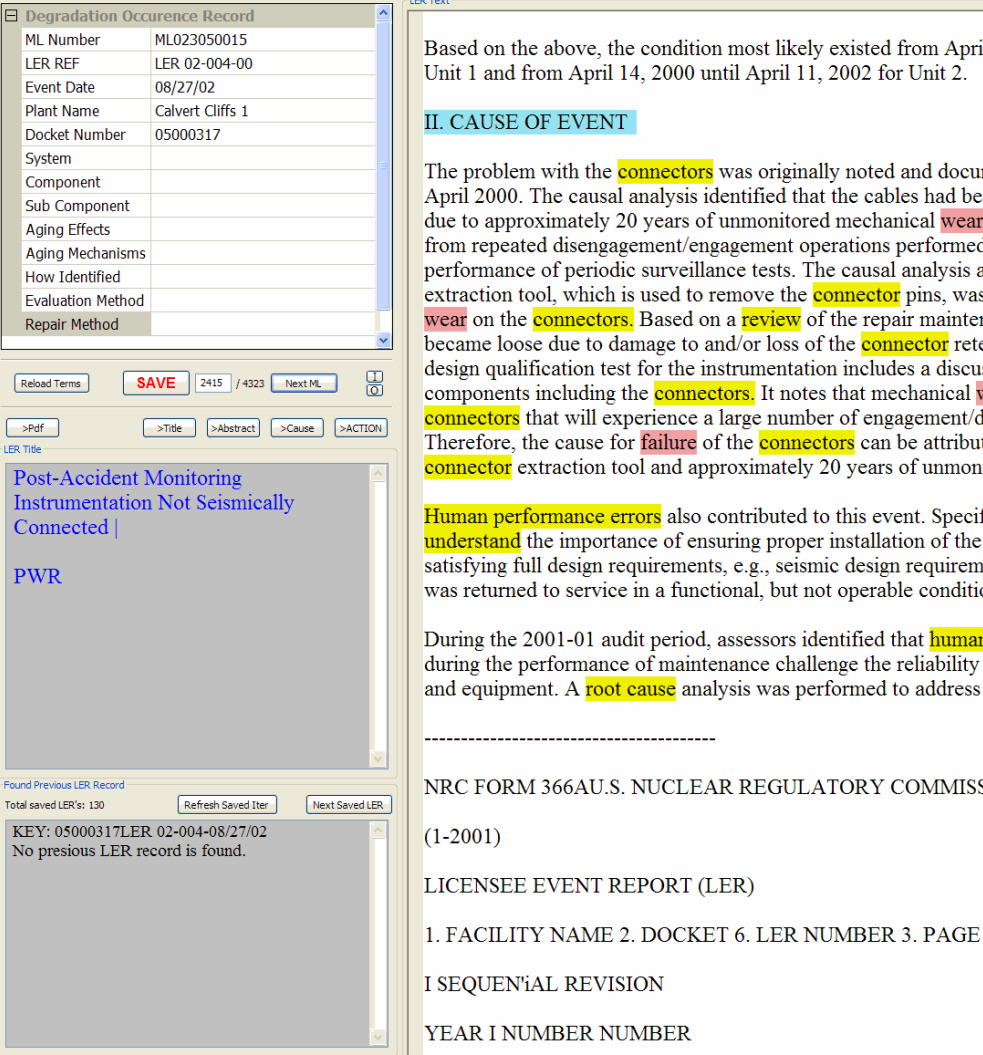

Figure 3-15 An LER Related to Human Error and Connectors 


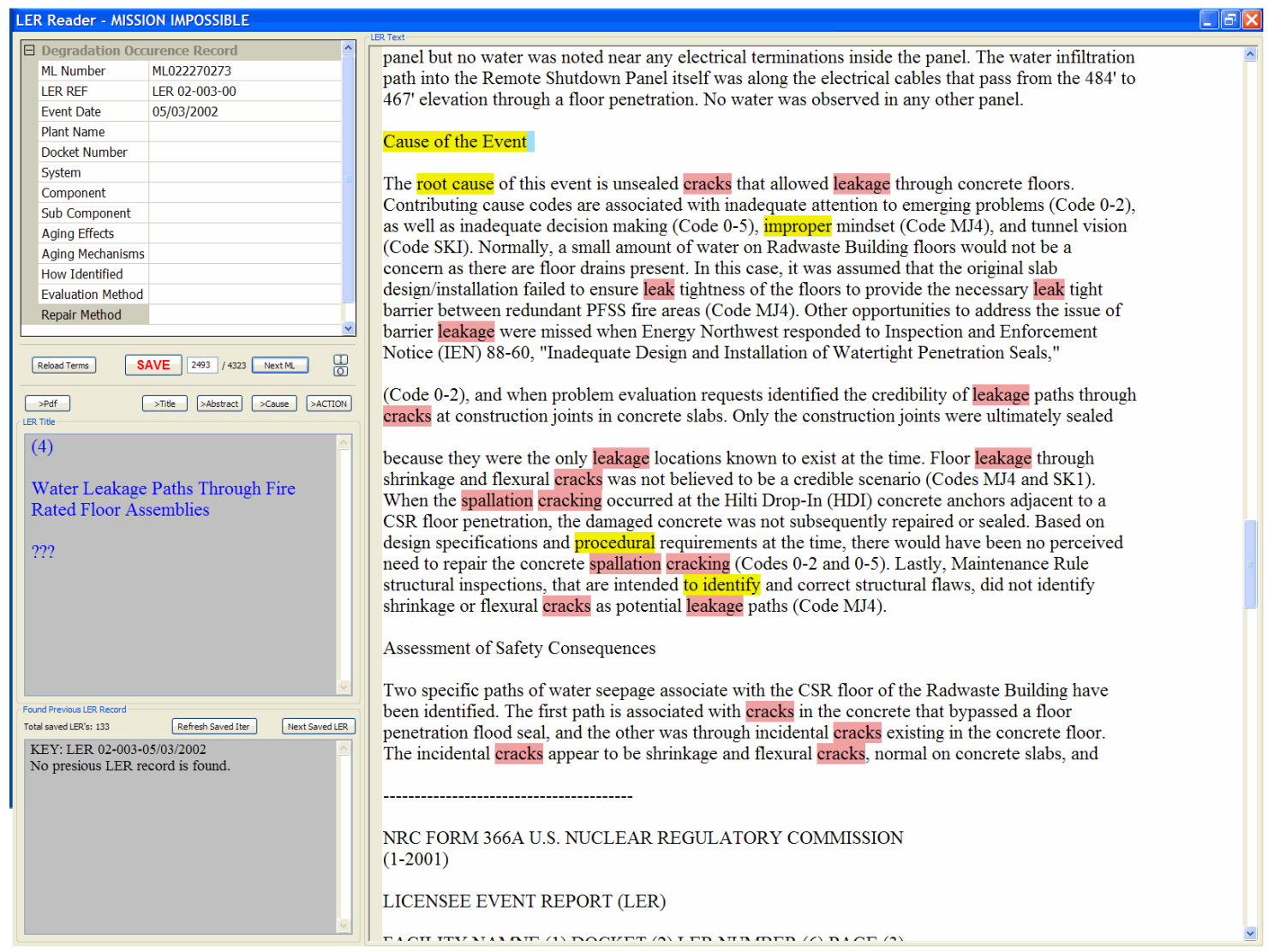

Figure 3-16 An LER Related to Concrete Degradation 


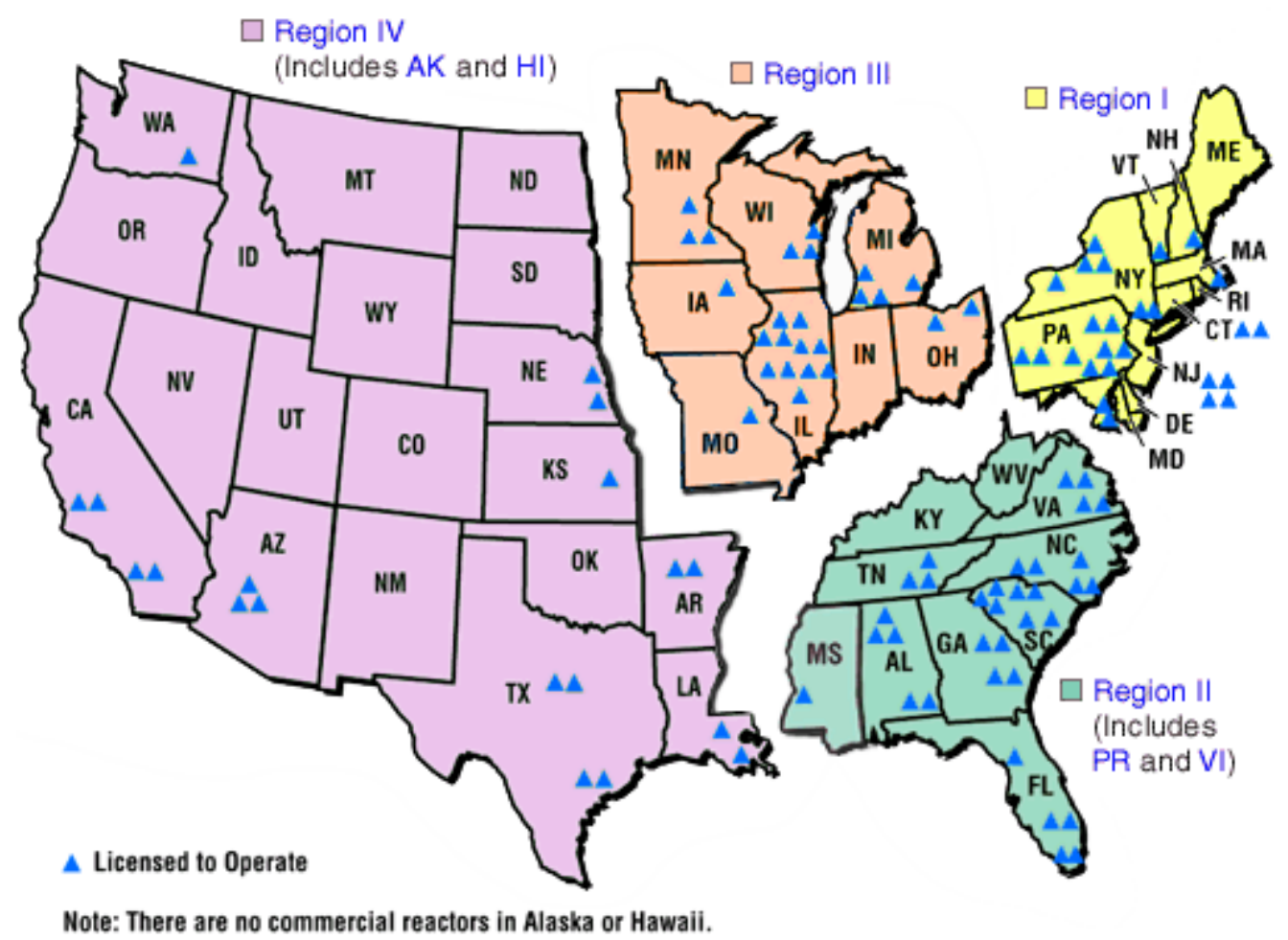

Figure 3-17 Distribution of 104 Operating US NPPs in Four NRC Regions [NRC Website]

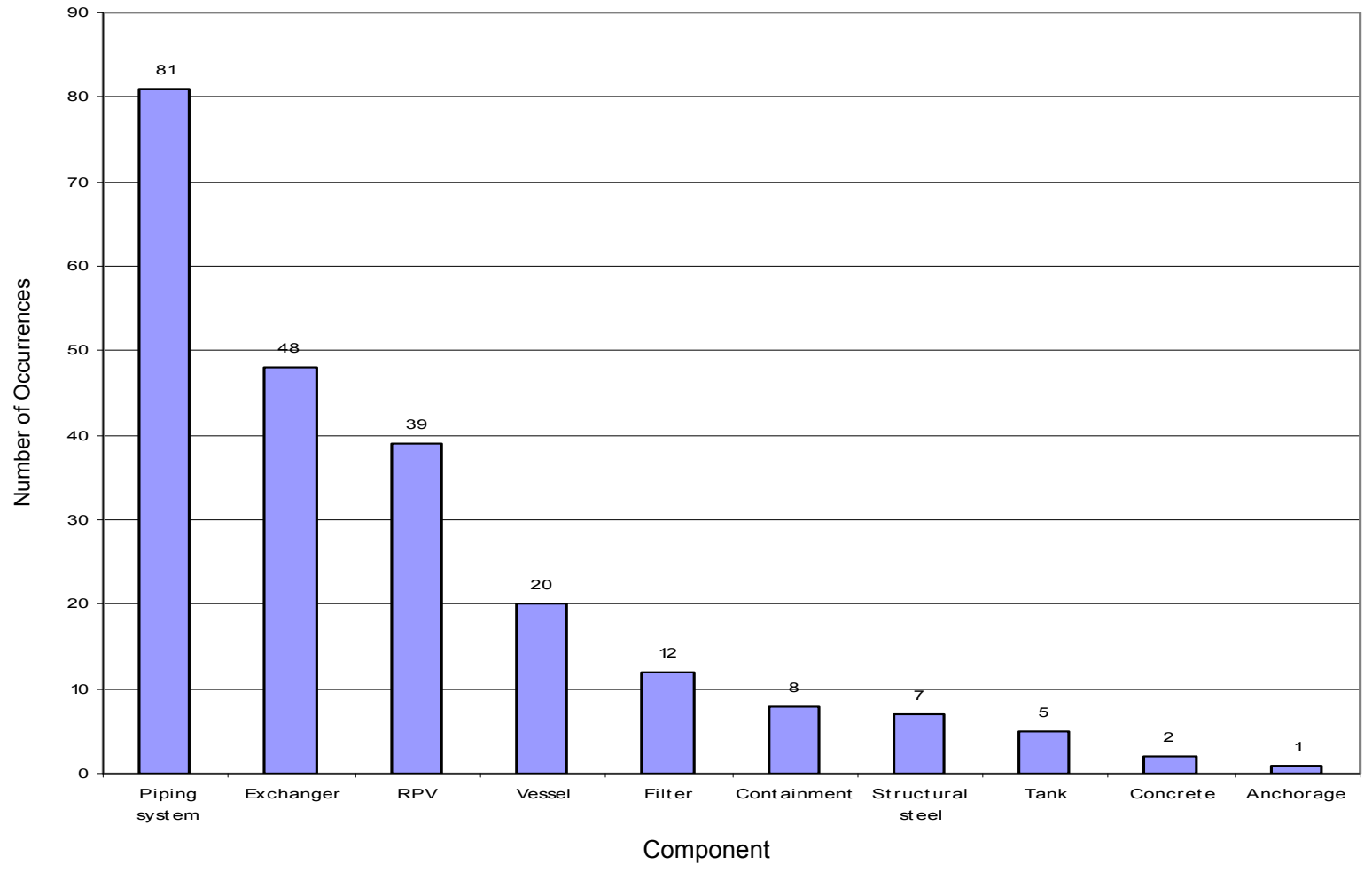

Figure 3-18 Distribution of SPC Degradation Occurrences over Component Category 


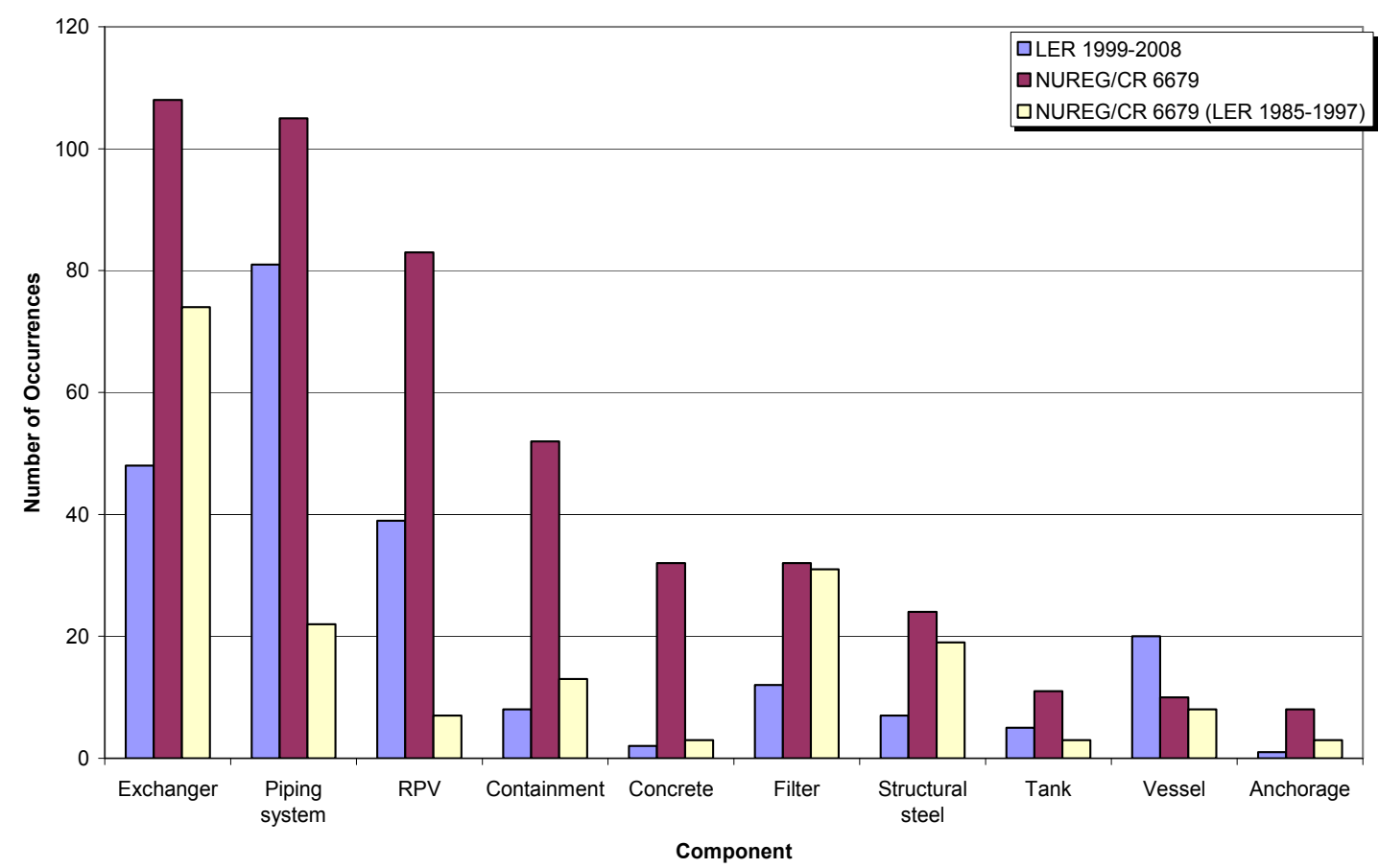

Figure 3-19 Distribution Comparison of SPC Degradation Occurrences over Component

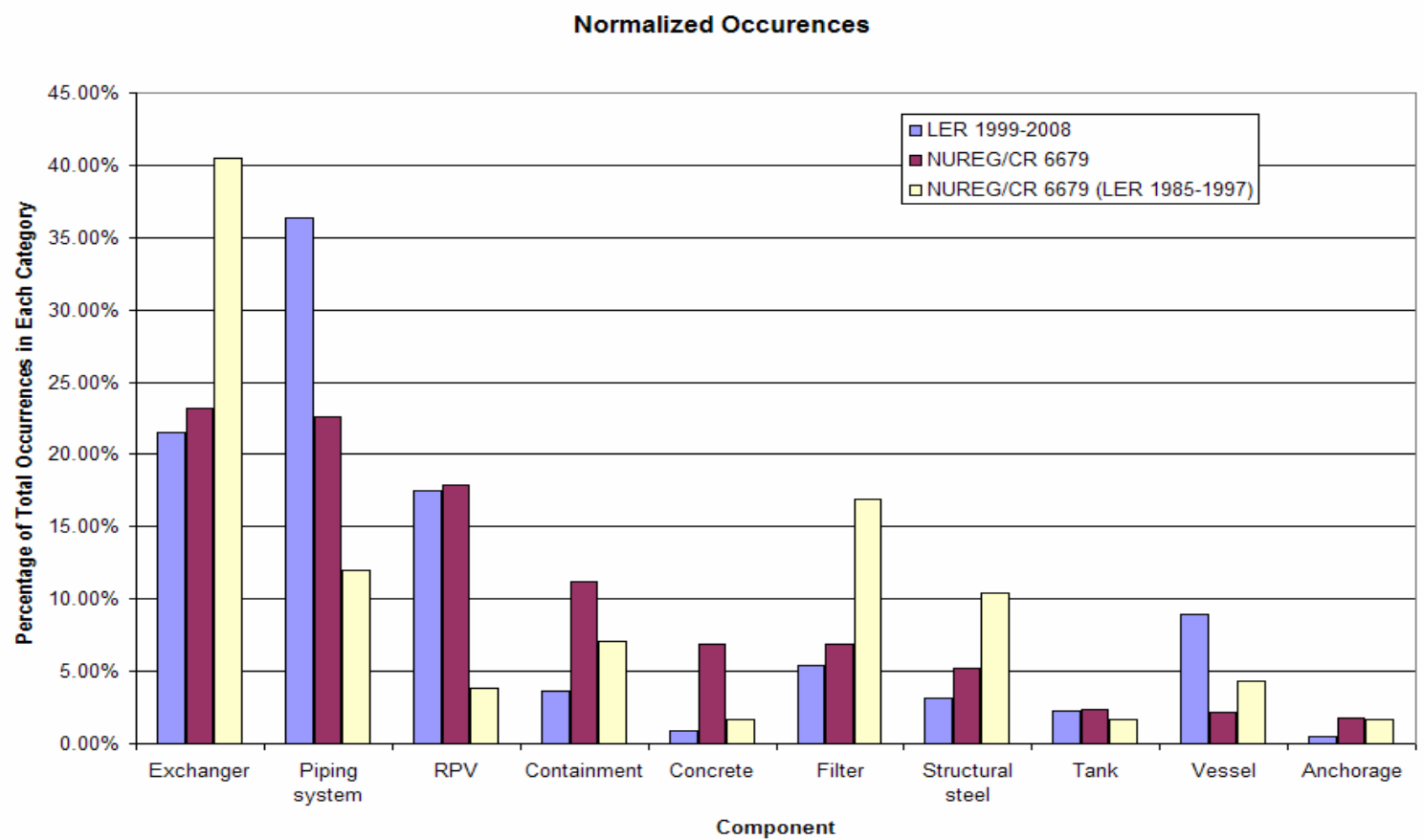

Figure 3-20 Comparison of Normalized Distribution of SPC Degradation Occurrences over Component 



Figure 3-21 Distribution Comparison of SPC Degradation Occurrences over Time 


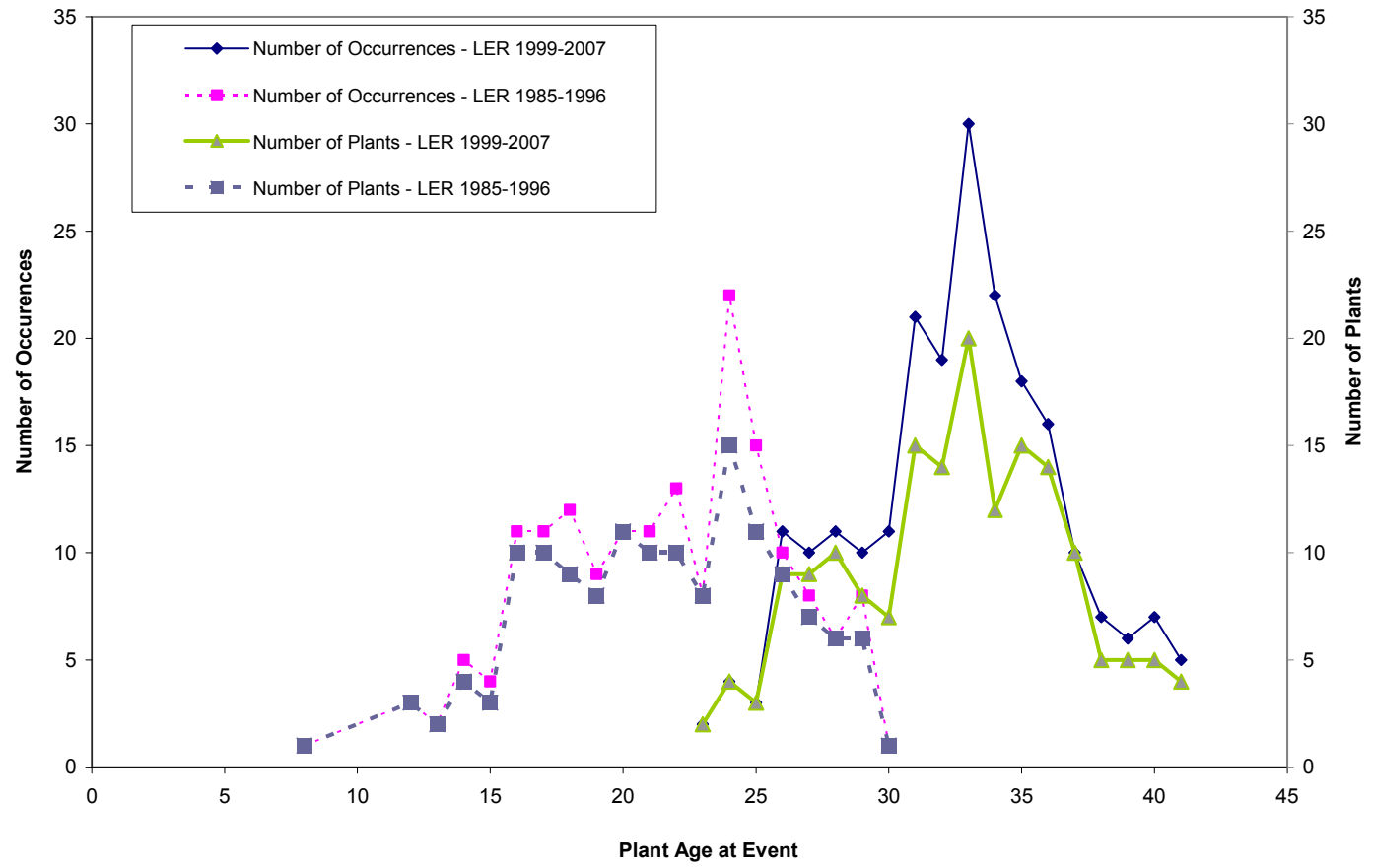

Figure 3-22 Number of Degradation Occurrences and NPP Units with Plant Age at Event

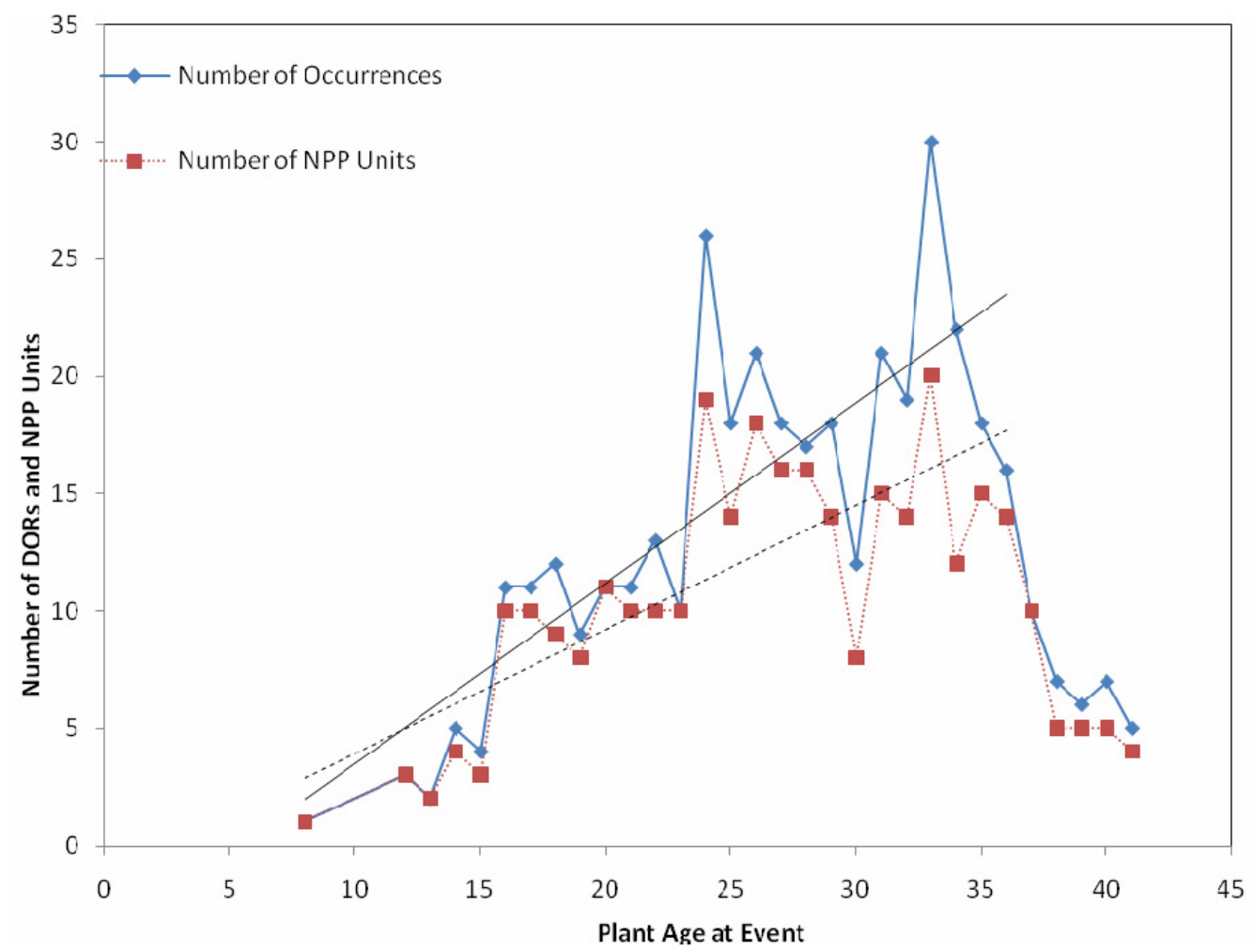

Figure 3-23 Number of Degradation Occurrences and NPP Units with PAAE for Combined Data Series 


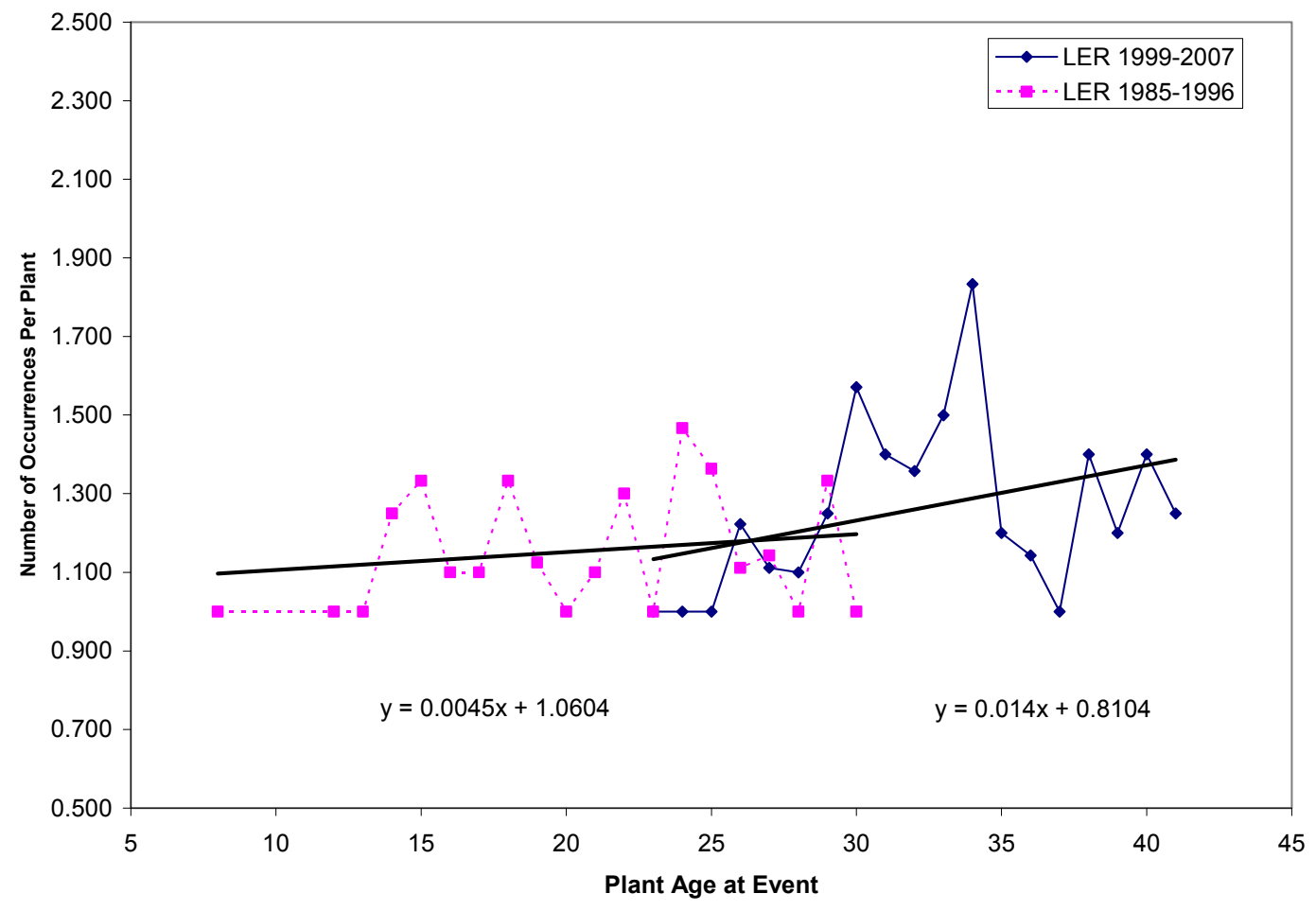

Figure 3-24 Average Degradation Occurrences with Plant Age at Event

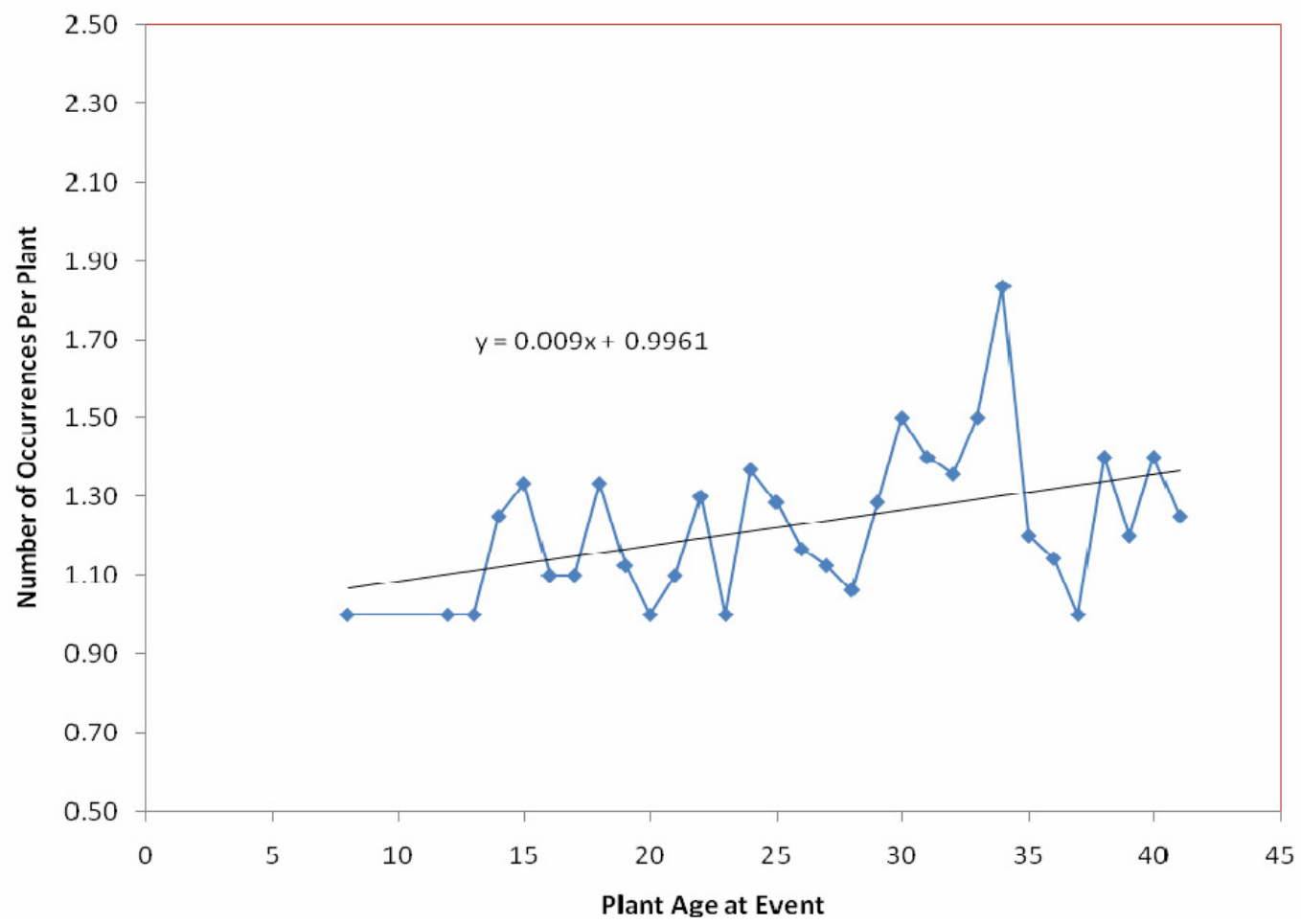

Figure 3-25 Relation of Average DORs per Plant versus PAAE with Combined Data Series 


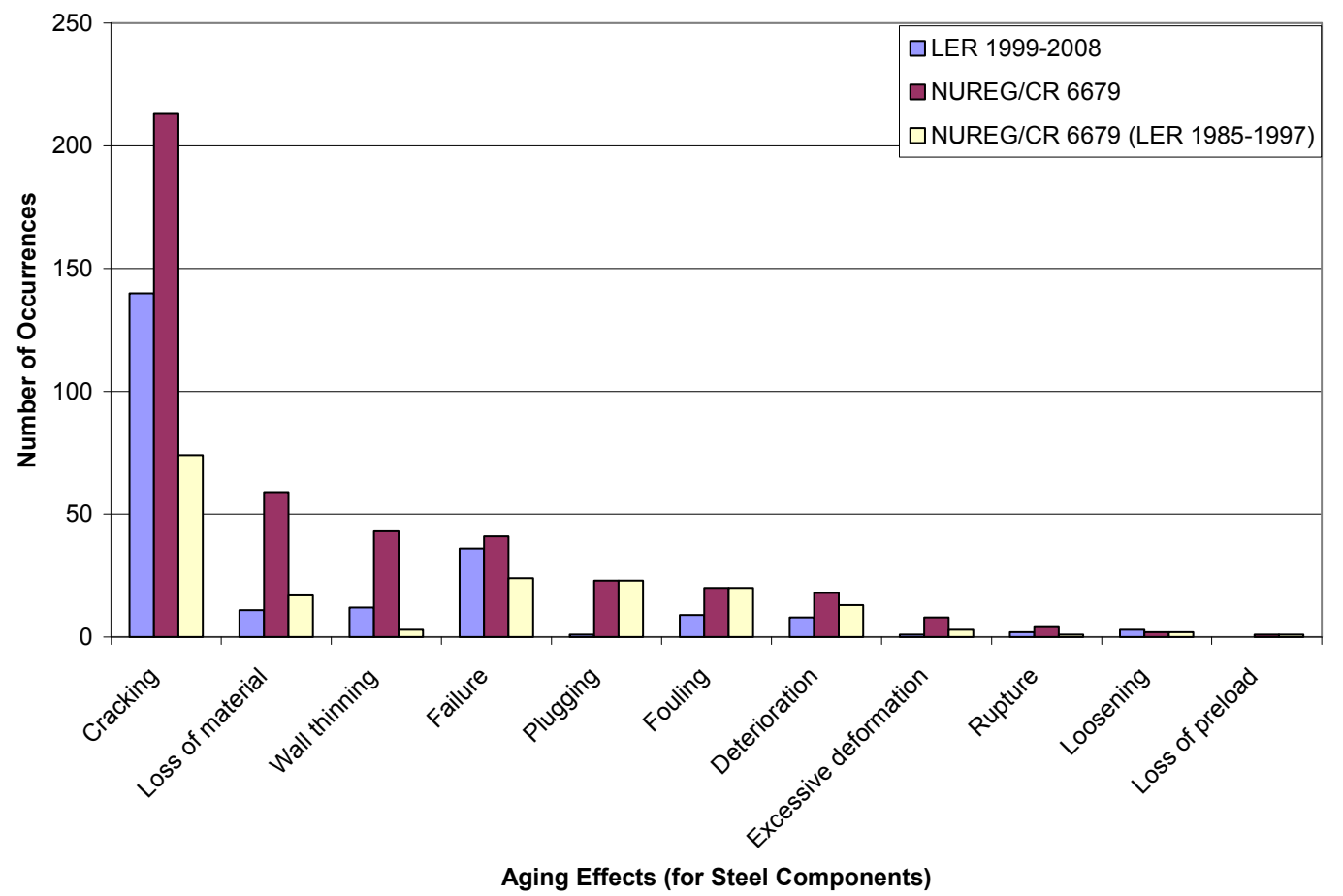

Figure 3-26 Distribution Comparison of SPC Degradation Occurrences over Aging Effect (for Steel Component)

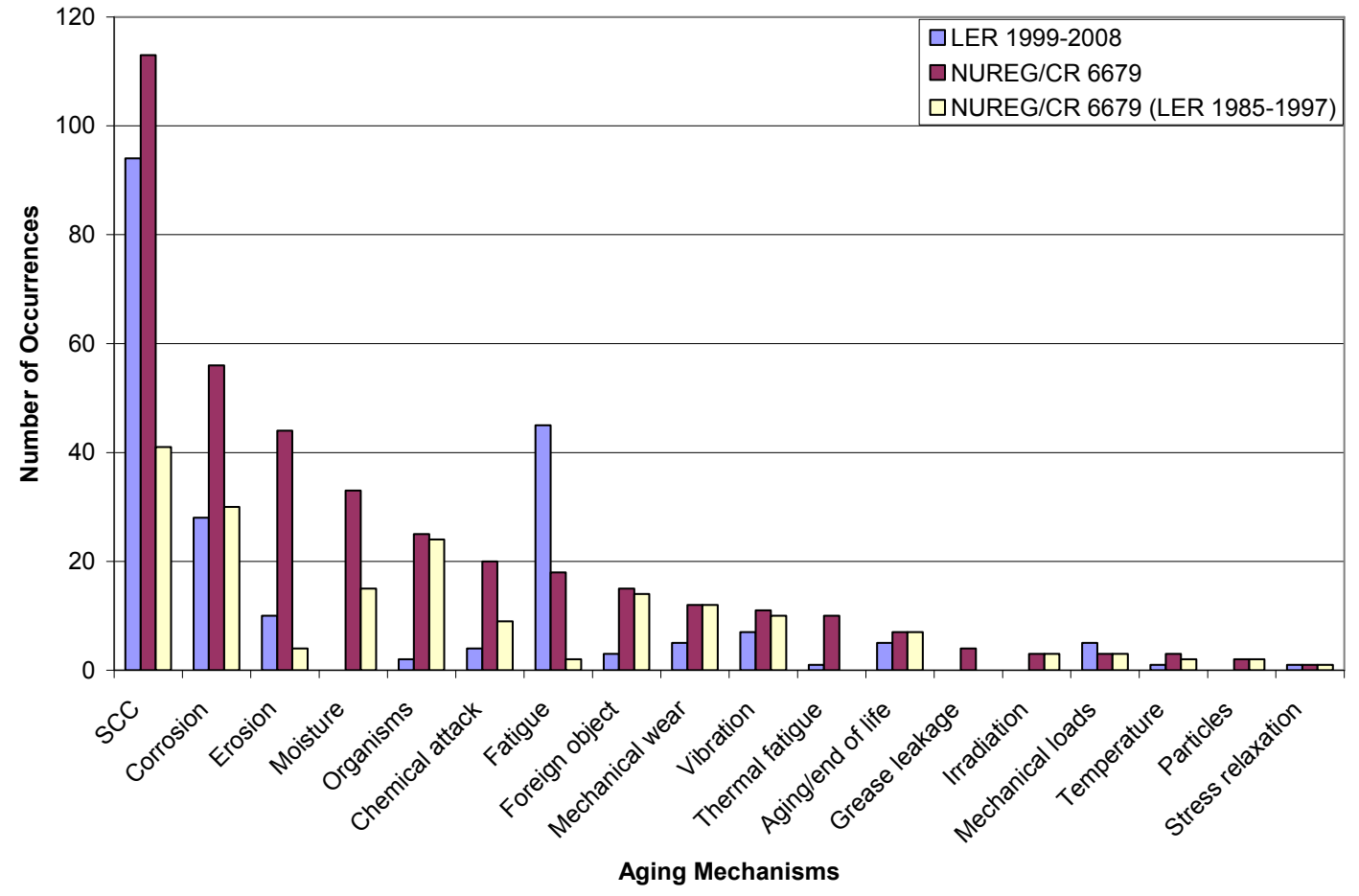

Figure 3-27 Distribution Comparison of SPC Degradation Occurrences over Aging Mechanism 


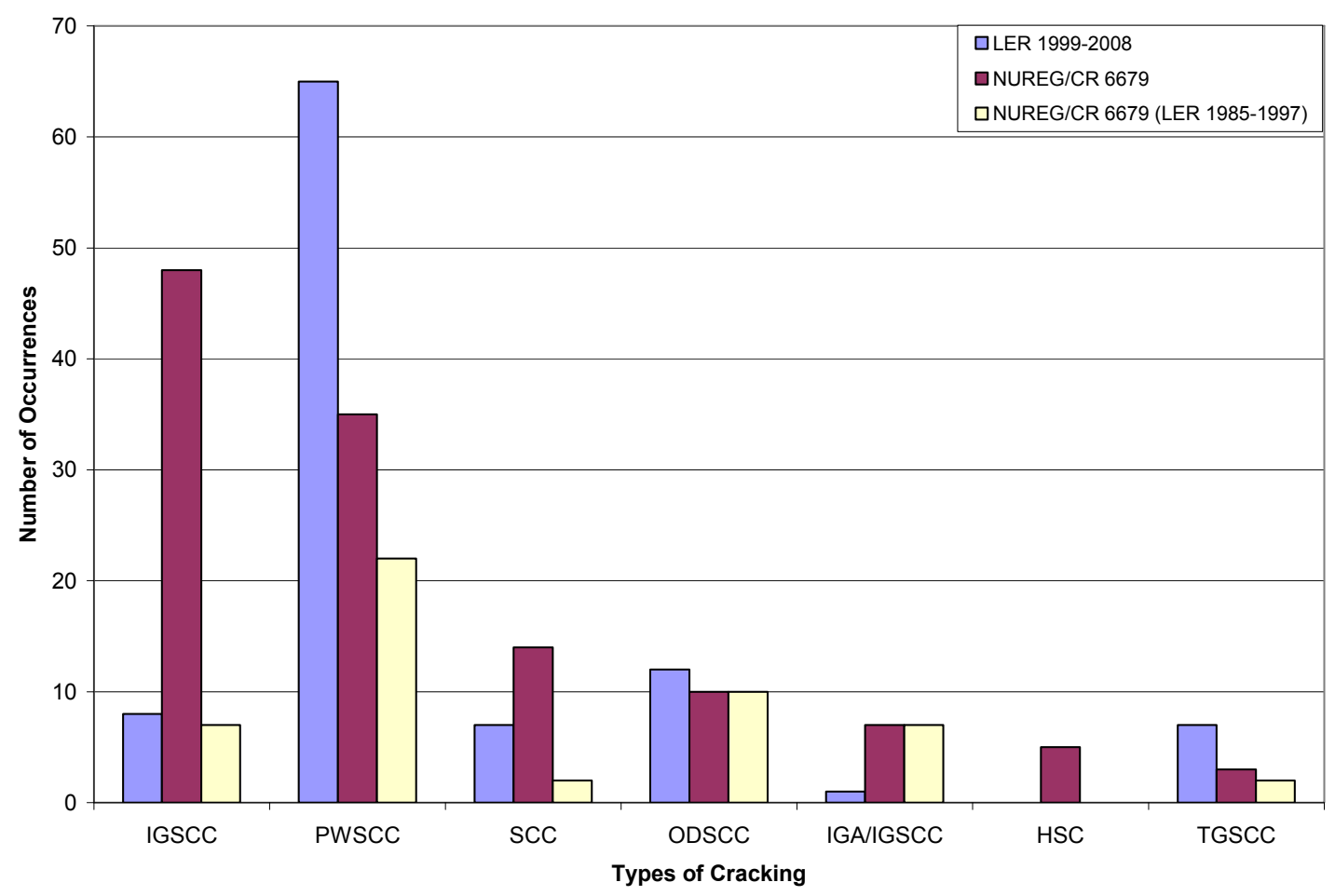

Figure 3-28 Distribution Comparison of SPC Degradation Occurrences over Cracking Type

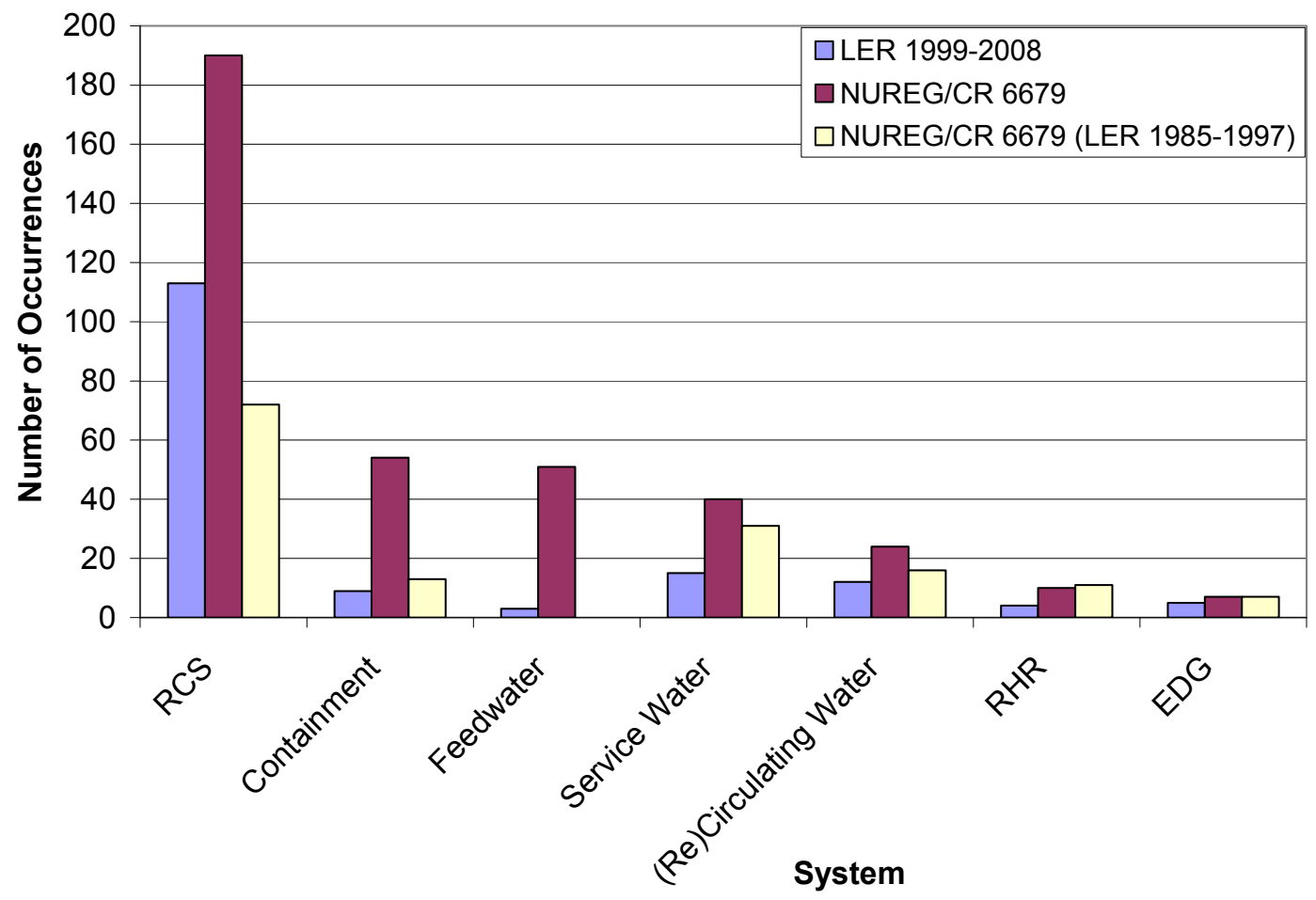

Figure 3-29 Distribution Comparison of SPC Degradation Occurrences over System 



\section{GENERIC COMMUNICATIONS AND LRAS}

In addition to recent LERs, selected generic communications and license renewal applications (LRAs) were also reviewed to identify the characteristics of the age-related degradation of structures and passive components (SPC). Generic communications reviewed included Generic Letters (GL), Bulletins (BL), and Information Notices (IN) issued by the US NRC. Since these documents may not include all information needed for the degradation occurrence record (DOR) or may include duplicate DORs with respect to LERs, the findings are not incorporated into the same DOR tables described in Section 3 of this report. Therefore, the degradation information obtained from NRC generic communications is evaluated and described separately in this section of the report.

LRAs were also reviewed under this study because they provide useful information about aging degradation of SPCs. These applications are required to include information about aging by regulations (10 CFR 50.73) which cover the submittal of LRAs. These regulations specifically require applicants to describe their operating experience regarding age-related degradation for the various SPCs.

\subsection{Generic Letters, Information Notices, and Bulletins}

Generic communications are publicly available documents through the NRC web site. Generic communications are the NRC's primary method of communicating a common need or resolution approach to an issue or providing guidance on issues pertaining to a matter of regulatory interest. Generic communications also allow the NRC to communicate and share industry experiences and send information to specific classes of licensees and interested stakeholders. The type of generic communication issued is determined during NRC evaluations of the operating nuclear industry and regulatory activities. Once issued, a generic communication is placed in the Agencywide Documents Access and Management System (ADAMS) as an official NRC record. The generic communication is then electronically sent out to subscribers and posted to the NRC external Web site.

Three types of generic communications were reviewed in this study. They consist of Generic Letters (GLs), Bulletins (BLs), and Information Notices (INs). GLs are NRC communications to licensees for the purpose of transmitting important information and usually require action or response. BLs address significant issues of great urgency and usually require action or response. INs relate to safety, safeguards, or environmental issues on which licensees consider action as appropriate.

Similar to the review effort of LERs, the focus in this research study was on recent GLs, BLs, and INs, within the general time periods of 1997 through the first part of 2008. Since degradation occurrences did not occur or were not identified on the NRC web site for some of these years, the actual periods of degradation were 1997 to 2006 for GLs, 2001 to 2004 for BLs, and 1998 to 2007 for INs. All of the generic communications during these periods were reviewed to identify and document aging degradation for the ten categories of structures and passive components (SPCs) listed in Section 3.1. Document titles were instructive in determining which documents should be retrieved for more careful review. A total of 46 generic communication documents were reviewed, including 7 GLs, 6 BLs, and 33 INs.

The seven reviewed GLs are listed in the following using their index numbers and titles (in descending order of time):

1. 2006-01, "Steam generator tube integrity and associated technical specifications" 
2. 2004-02, "Potential impact of debris blockage on emergency recirculation during design basis accidents at pressurized-water reactors"

3. 2004-01, "Requirements for steam generator tube inspection"

4. 98-04, "Potential for Degradation of the Emergency Core Cooling System and the Containment Spray System After a Loss-of-Coolant Accident Because of Construction and Protective Coating Deficiencies and Foreign Material in Containment"

5. 97-06 (1997), "Degradation of Steam Generator Internals"

6. 97-05 (1997), "Steam Generator Tube Inspection Techniques"

7. 97-01 (1997), "Degradation of Control Rod Drive Mechanism Nozzle and Other Vessel Closure Head Penetrations"

Similarly, the six reviewed BLs are listed below (in descending order of time):

1. 2004-01, "Inspection of Alloy 82/182/600 Materials Used in the Fabrication of Pressurizer Penetrations and Steam Space Piping Connections at Pressurized-Water Reactors"

2. 2003-02, "Leakage from Reactor Pressure Vessel Lower Head Penetrations and Reactor Coolant Pressure Boundary Integrity"

3. 2003-01, "Potential Impact of Debris Blockage on Emergency Sump Recirculation at Pressurized-Water Reactors"

4. 2002-02, "Reactor Pressure Vessel Head and Vessel Head Penetration Nozzle Inspection Programs"

5. 2002-01, "Reactor Pressure Vessel Head Degradation and Reactor Coolant Pressure Boundary Integrity"

6. 2001-01, "Circumferential Cracking of Reactor Pressure Vessel Head Penetration Nozzles"

The 33 reviewed INs are listed as well (in descending order of time):

1. 2007-37, "Buildup of Deposits in Steam Generator"

2. 2007-21, "Pipe Wear Due to Interaction of Flow-induced Vibration and Reflective Metal Insulation"

3. 2006-27, "Circumferential Cracking in the Stainless Steel Pressurizer Heater Sleeves of Pressurized Water Reactors"

4. 2006-17, "Recent Operating Experience of Service Water Systems Due to External Conditions"

5. 2006-08, "Secondary Piping Rupture at the Mihama Power Station in Japan"

6. 2006-01, "Torus Cracking in a BWR Mark I Containment"

7. 2004-21, "Additional Adverse Effect of Boric Acid Leakage: Potential Impact on PostAccident Coolant $\mathrm{pH}$ "

8. 2004-11, "Cracking in Pressurizer Safety and Relief Nozzles and in Surge Line Nozzle"

9. 2004-09, "Corrosion of Steel Containment and Containment Liner"

10. 2004-08, "Reactor Coolant Pressure Boundary Leakage Attributable to Propagation of Cracking in Reactor Vessel Nozzle Welds" 
11. 2004-05, "Spent Fuel Pool Leakage to Onsite Groundwater"

12. 2004-01, "Auxiliary Feedwater Pump Recirculation Line Orifice Fouling - Potential Common Cause Failure"

13. 2003-13, "Steam Generator Tube Degradation at Diablo Canyon"

14. 2003-11s1 and original 2003-11, "Leakage Found on Bottom-Mounted Instrumentation Nozzles"

15. 2003-08, "Potential Flooding Through Unsealed Concrete Floor Cracks"

16. 2003-05, "Failure to Detect Freespan Cracks in PWR Steam Generator Tubes"

17. 2003-02, "Recent Experience With Reactor Coolant System Leakage And Boric Acid Corrosion"

18. 2002-26 s1, s2, and original 2002-26, "Additional Flow-Induced Vibration Failures after a Recent Power Uprate"

19. 2002-02 s1, "Recent Experience With Plugged Steam Generator Tubes"

20. 2002-21 s1 and original 2002-21, "Axial Outside-Diameter Cracking Affecting Thermally Treated Alloy 600 Steam Generator Tubing"

21. 2002-13, "Possible Indicators of Ongoing Reactor Pressure Vessel Head Degradation"

22. 2002-11, "Recent Experience with Degradation of Reactor Pressure Vessel Head"

23. 2002-02, "Recent Experience with Plugged Steam Generator Tubes"

24. 2001-16, "Recent Foreign and Domestic Experience with Degradation of Steam Generator Tubes and Internals"

25. 2001-09, "Main Feedwater System Degradation in Safety-Related ASME Code Class 2 Piping Inside the Containment of a Pressurized Water Reactor"

26. 2001-05, "Through-Wall Circumferential Cracking of Reactor Pressure Vessel Head Control Rod Drive Mechanism Penetration Nozzles at Oconee Nuclear Station, Unit 3"

27. 2000-17 s1, s2, and original 2000-17, "Crack in Weld Area of Reactor Coolant System Hot Leg Piping at V. C. Summer"

28. 2000-09, "Steam Generator Tube Failure at Indian Point Unit 2"

29. 1999-10, Rev. 1 and 1999-10, "Degradation of Prestressing Tendon Systems in Prestressed Concrete Containments"

30. 1998-45, "Cavitation Erosion of Letdown Line Orifices Resulting in Fatigue Cracking of Pipe Welds"

31. 1998-27, "Steam Generator Tube End Cracking"

32. 1998-26, "Settlement Monitoring and Inspection of Plant Structures Affected by Degradation of Porous Concrete Subfoundations"

33. 1998-11, "Cracking of Reactor Vessel Internal Baffle Former Bolts in Foreign Plants"

A summary for each of these documents is presented in Table 4-1, which includes additional information under the heading "Topic" and "SPC Affected." Table 4-2 summarizes for each type of generic communication the number of generic communications that address a particular component / subcomponent, and Table 4-3 aggregates the results in Table 4-2 by removing the types of generic communications. It should be noted that the number of generic communications addressing a component is different from the number of degradation occurrences because one generic correspondence may refer to multiple events with regard to one component. As can be seen from Table 4-3, steam generators (exchangers), RPVs, and Piping systems are the top three components with the greatest number of generic correspondences, which are about $28 \%, 28 \%$, and $15 \%$ of the total 46 documents. Although these numbers do not equal to what have been 
found using LERs as described in Section 3, for reasons as stated above, they constitute a total of $71 \%$, which is very similar to $75 \%$ found in Section 3. There are 6 generic correspondences on structural type components (containment, concrete, spent fuel pool), which is about $13 \%$ of the total number of reviewed generic correspondences. This ratio is just slightly higher than the $8 \%$ as determined in Section 3, indicating that generic communications addressed more structural type components.

In summary, the results of the review of generic letters generally confirm what has been found or explained in Section 3 for LER evaluations.

\subsection{License Renewal Applications}

The Atomic Energy Act and NRC regulations limit commercial power reactor licenses to an initial 40 years but also permit such licenses to be renewed. This original 40 -year term for reactor licenses was based on economic and antitrust considerations - not on limitations of nuclear technology. Due to this selected period, however, some structures and components may have been engineered on the basis of an expected 40-year service life. Since the expense of design and construction of NPPs has been so great, it is generally much more cost effective to extend the operating life of a NPP beyond the 40-year license given to plants. Therefore, the NRC has established a timely license renewal process and clear requirements for renewing the operating license of NPPs. These requirements are codified in 10 CFR Part 54, "Requirements for Renewal of Operating Licenses For Nuclear Power Plants" and 10 CFR Part 51, "Environmental Protection Regulations for Domestic Licensing and Related Regulatory Functions." These requirements assure safe plant operation for the extended plant life. Renewal of license, when approved is granted for an additional 20 years.

Documents related to LRA are useful because they identify applicable aging effects and operating experience which would describe aging degradation of structures and components at the plants. At the NRC website, there were 24 completed (reviewed and approved) applications for license renewal. These are presented below.

- Calvert Cliffs, Units 1 and 2

- Oconee Nuclear Station, Units 1, 2 and 3

- Arkansas Nuclear One, Unit 1

- Edwin I. Hatch Nuclear Plant, Units 1 and 2

- Turkey Point Nuclear Plant, Units 3 and 4

- North Anna, Units 1 and 2, and Surry, Units 1 and 2

- Peach Bottom, Units 2 and 3

- St. Lucie, Units 1 and 2

- Fort Calhoun Station, Unit 1

- McGuire, Units 1 and 2, and Catawba, Units 1 and 2

- H.B. Robinson Nuclear Plant, Unit 2

- R.E. Ginna Nuclear Power Plant, Unit 1

- V.C. Summer Nuclear Station, Unit 1

- Dresden, Units 2 and 3, and Quad Cities, Units 1 and 2

- Farley, Units 1 and 2

- Arkansas Nuclear One, Unit 2

- D.C. Cook, Units 1 and 2

- Millstone, Units 2 and 3

- Point Beach, Units 1 and 2 (BWR)

- Browns Ferry, Units 1, 2, and 3

- Brunswick, Units 1 and 2 
- Nine Mile Point, Units 1 and 2

- Monticello

- Palisades (PWR)

There are also 12 LRAs under review at the time that this study was performed and these are listed below.

- Oyster Creek - Application received July 22, 2005

- Pilgrim 1 - Application received January 27, 2006

- Vermont Yankee - Application received January 27, 2006

- James A. FitzPatrick - Application received August 1, 2006

- Susquehanna - Application received September 15, 2006

- Wolf Creek - Application received October 4, 2006

- Harris - Application received November 16, 2006

- Indian Point - Application received April 30, 2007

- Vogtle - Application received June 29, 2007

- Beaver Valley - Application received August 28, 2007

- Three Mile Island, Unit 1 - Application received January 8, 2008

- Prairie Island - Application received April 15, 2008

Since each LRA is quite large and the total number of LRAs are too numerous to review in the current study, two LRAs were selected for review in this study. The criteria used for selection of the two LRAs are as follows:

- One of each PWR \& BWR

- Recent LRA - more likely to reflect knowledge gained from prior LRA submittals \& comments from NRC

- More complete information on Operating Experience

- More detailed

Using these criteria, the two LRAs selected are Palisades (PWR) and Point Beach Units 1 \& 2 (BWR). The content of LRAs is listed below, with a partial aging management program list shown under the heading Appendix. As indicated earlier, this information is specifically required by 10 CFR Part 54. More information about LRAs and the content of the application is given in Section 2.1 of this report.

- Administrative Information

- Scoping and Screening Methodology for Identifying Structures and Components Subject to Aging Management Review, and Implementation Results

- Aging Management Review Results

- Time-Limited Aging Analyses

- Appendix - Aging Management Programs Alloy 600 Inspection Program ASME Section XI, Subsections IWB, IWC, IWD, IWF Inservice Inspection Program Bolting Integrity Program Boric Acid Corrosion Program Buried Services Corrosion Monitoring Program Closed Cycle Cooling Water Program Containment Inservice Inspection Program Containment Leakage Testing Program Others 
For each Aging Management Program (AMP) included in an LRA, there is an "Operating Experience" discussion that is very informative for aging-related degradation review. However, the information presented in these operating experience discussions is not as detailed as in the LERs.

Table 4-4 and Table 4-5 present a detailed review of these two LRAs, which include summaries of the AMP and operating experiences. The operating experience for each AMP discusses industry-wide observations and plant specific issues. The information presented in Tables 4-4 and 4-5 are summaries of the actual publicly available LRAs presented on the NRC web site. These summaries are excerpts of the AMPs and operating experience obtained from the LRAs and are shortened/edited to convey the important elements needed for this study. As the information is not considered complete enough for a direct comparison to the results using LERs, information in these two tables are used qualitatively. As can be seen in the tables, degradation in components related to piping systems, RPVs, and exchangers occur more often than the other components being studied. This observation is consistent with the results from the review of LERs because the industry and plant-specific operating experience on aging in the LRAs was most likely already in the LERs that the plant(s) submitted in the past. However, the LRAs would have identified additional cases of degradation if they would have occurred. 
Table 4-1 NRC Generic Communications for Information Related to Aging Degradation

\section{[Available on the NRC Web Site]}

\begin{tabular}{|c|c|c|c|c|}
\hline Type & No. & Title & Topic & $\begin{array}{c}\text { SPC } \\
\text { Affected }\end{array}$ \\
\hline $\begin{array}{l}\text { Generic } \\
\text { Letter } \\
(\mathrm{GL})\end{array}$ & 2006-01 & $\begin{array}{l}\text { Steam } \\
\text { Generator } \\
\text { Tube } \\
\text { Integrity } \\
\text { and } \\
\text { Associated } \\
\text { Technical } \\
\text { Specificati } \\
\text { ons }\end{array}$ & $\begin{array}{l}\text { The U.S. Nuclear Regulatory Commission (NRC) is concerned that current TS requirements may not } \\
\text { be sufficient to ensure that steam generator (SG) tube integrity can be maintained in accordance with } \\
\text { current licensing and design basis. The NRC is, therefore, issuing this GL to request that addressees } \\
\text { either submit a description of their program for ensuring SG tube integrity for the interval between } \\
\text { inspections or adopt alternative TS requirements for ensuring SG tube integrity. Alternative TS } \\
\text { requirements that address the staff's concerns with the existing TS were developed by the industry and } \\
\text { found acceptable by the staff. } \\
\text { Related Generic Communications } \\
\text { - NRC Information Notice 2005-09, "Indications in Thermally Treated Alloy } 600 \text { Steam Generator } \\
\text { Tubes and Tube-to-Tubesheet Welds," April 7, } 2005 \text { (ML050530400). } \\
\text { - NRC Information Notice 2004-17, "Loose Part Detection and Computerized Eddy Current Data } \\
\text { Analysis in Steam Generators," August 25, 2004 (ML042180094). } \\
\text { - NRC Information Notice 2004-16, "Tube Leakage due to a Fabrication Flaw in a Replacement Steam } \\
\text { Generator," August 3, 2004 (ML041460357). } \\
\text { - NRC Information Notice 2004-10, "Loose Parts in Steam Generators," May 4, 2004 } \\
\text { (ML041170480). } \\
\text { - NRC Generic Letter 2004-01, "Requirements for Steam Generator Tube Inspections,"August 30, } \\
2004 \text { (ML042370766). }\end{array}$ & $\begin{array}{l}\text { Steam } \\
\text { generators }\end{array}$ \\
\hline GL & $2004-02$ & $\begin{array}{l}\text { Potential } \\
\text { Impact of } \\
\text { Debris } \\
\text { Blockage } \\
\text { on } \\
\text { Emergency } \\
\text { Recirculati } \\
\text { on during } \\
\text { Design } \\
\text { Basis } \\
\text { Accidents }\end{array}$ & $\begin{array}{l}\text { The U.S. Nuclear Regulatory Commission (NRC) is issuing this generic letter to: } \\
\text { (1) Request that addressees perform an evaluation of the emergency core cooling system (ECCS) and } \\
\text { containment spray system (CSS) recirculation functions in light of the information provided in this } \\
\text { letter and, if appropriate, take additional actions to ensure system function. Additionally, addressees } \\
\text { are requested to submit the information specified in this letter to the NRC. This request is based on the } \\
\text { identified potential susceptibility of pressurized-water reactor (PWR) recirculation sump screens to } \\
\text { debris blockage during design basis accidents requiring recirculation operation of ECCS or CSS and } \\
\text { on the potential for additional adverse effects due to debris blockage of flowpaths necessary for ECCS } \\
\text { and CSS recirculation and containment drainage. } \\
\text { (2) Require addressees to provide the NRC a written response in accordance with } 10 \text { CFR 50.54(f). }\end{array}$ & Strainers \\
\hline
\end{tabular}




\begin{tabular}{|c|c|c|c|c|}
\hline Type & No. & Title & Topic & $\begin{array}{c}\text { SPC } \\
\text { Affected }\end{array}$ \\
\hline & & $\begin{array}{l}\text { at } \\
\text { Pressurized } \\
\text {-Water } \\
\text { Reactors }\end{array}$ & 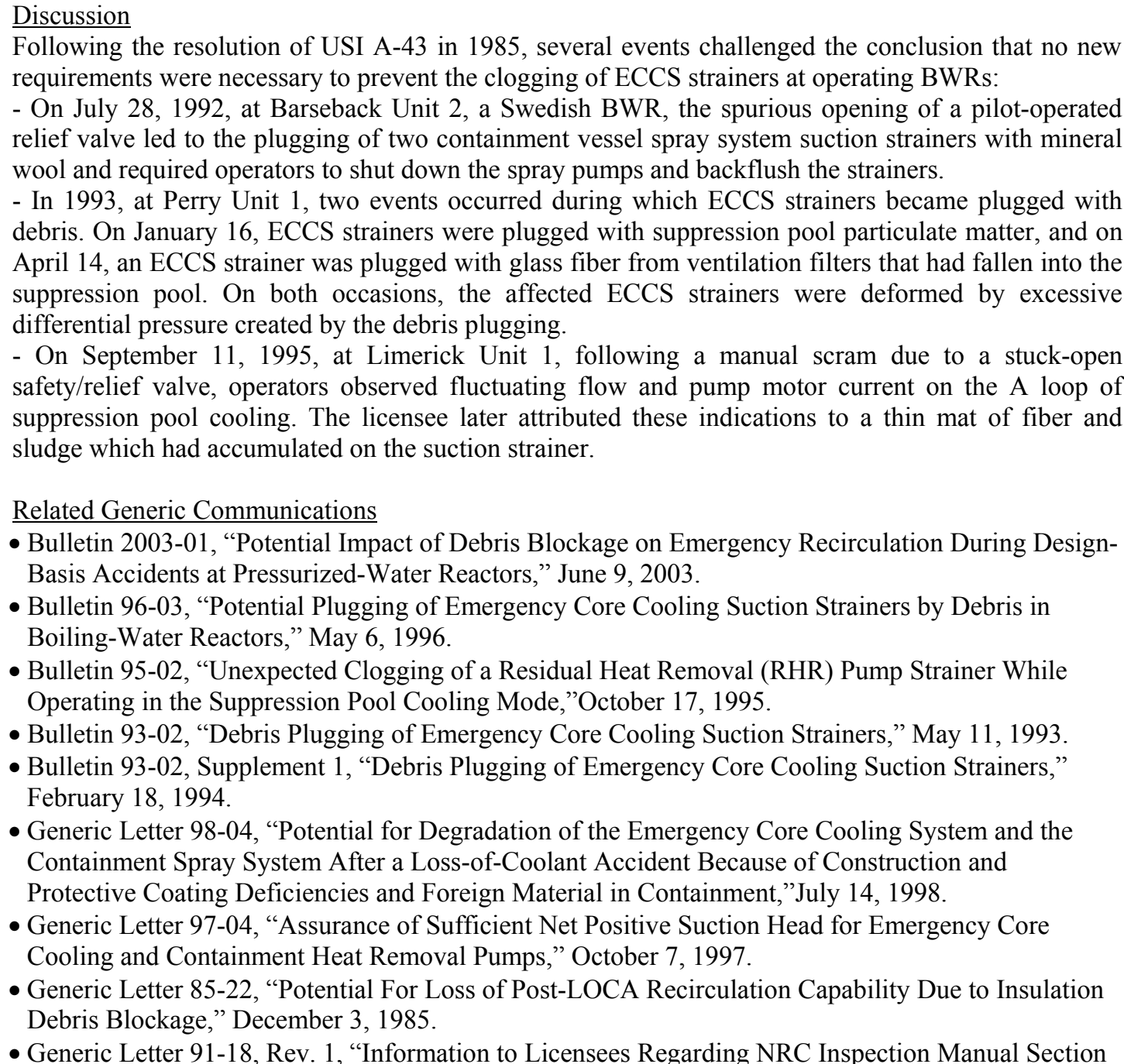 & \\
\hline
\end{tabular}




\begin{tabular}{|c|c|c|c|c|}
\hline Туре & No. & Title & Topic & $\begin{array}{c}\text { SPC } \\
\text { Affected }\end{array}$ \\
\hline & & & 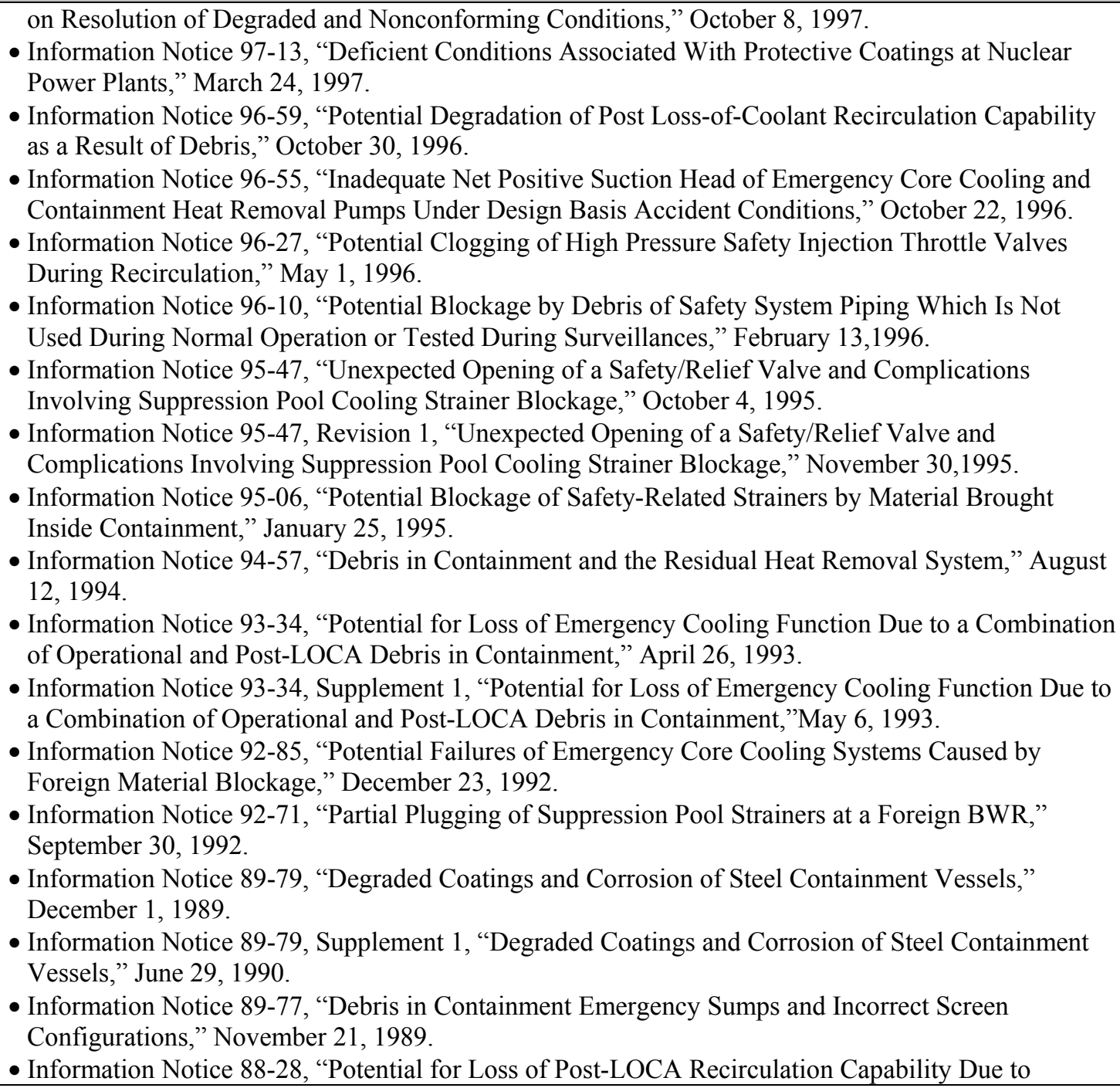 & \\
\hline
\end{tabular}




\begin{tabular}{|c|c|c|c|c|}
\hline Type & No. & Title & Topic & $\begin{array}{c}\text { SPC } \\
\text { Affected }\end{array}$ \\
\hline & & & Insulation Debris Blockage," May 19, 1988. & \\
\hline GL & $2004-01$ & $\begin{array}{l}\text { Requireme } \\
\text { nts for } \\
\text { Steam } \\
\text { Generator } \\
\text { Tube } \\
\text { Inspec }\end{array}$ & 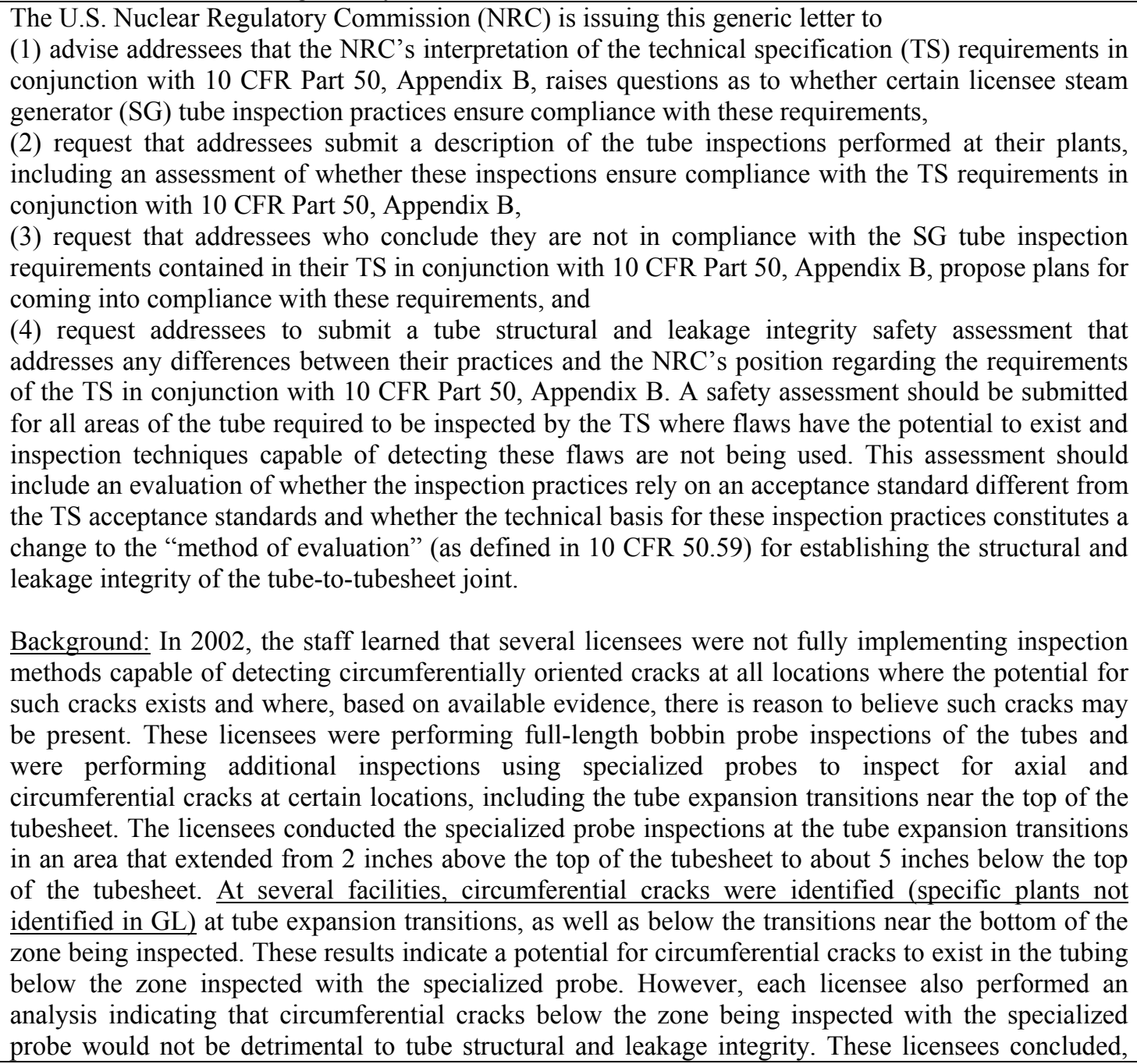 & $\begin{array}{l}\text { Steam } \\
\text { Generators }\end{array}$ \\
\hline
\end{tabular}




\begin{tabular}{|c|c|c|c|c|}
\hline Туре & No. & Title & Topic & $\begin{array}{c}\text { SPC } \\
\text { Affected }\end{array}$ \\
\hline & & & $\begin{array}{l}\text { therefore, that additional inspections for circumferential cracks with the specialized probe were } \\
\text { unnecessary. These analyses had not been provided to the NRC staff. }\end{array}$ & \\
\hline GL & $98-04$ & $\begin{array}{l}\text { Potential } \\
\text { for } \\
\text { Degradatio } \\
\mathrm{n} \text { of the } \\
\text { Emergency } \\
\text { Core } \\
\text { Cooling } \\
\text { System and } \\
\text { the } \\
\text { Containme } \\
\text { nt Spray } \\
\text { System } \\
\text { After a } \\
\text { Loss-of- } \\
\text { Coolant } \\
\text { Accident } \\
\text { Because of } \\
\text { Constructio } \\
\mathrm{n} \quad \text { and } \\
\text { Protective } \\
\text { Coating } \\
\text { Deficiencie } \\
\mathrm{s} \quad \text { and } \\
\text { Foreign } \\
\text { Material in } \\
\text { Containme } \\
\text { nt }\end{array}$ & $\begin{array}{l}\text { The U.S. Nuclear Regulatory Commission (NRC) is issuing this generic letter for several reasons. It } \\
\text { alerts addressees that foreign material continues to be found inside operating nuclear power plant } \\
\text { containments. During a design basis loss-of-coolant accident (DB LOCA), this foreign material could } \\
\text { block an emergency core cooling system (ECCS) or safety-related containment spray system (CSS) } \\
\text { flow path or damage ECCS or safety-related CSS equipment. In addition, construction deficiencies } \\
\text { and problems with the material condition of ECCS systems, structures, and components (SSCs) inside } \\
\text { the containment continue to be found. Design deficiencies also have been found which could degrade } \\
\text { the ECCS or safety-related CSS. No action or information is requested regarding these issues. The } \\
\text { NRC has issued many previous generic communications on this subject, as discussed later in this } \\
\text { generic letter, and assumes that addressees have had adequate prior notice to consider possible actions } \\
\text { at their facilities to address these concerns. } \\
\text { The NRC is also issuing this generic letter to alert the addressees to the problems associated with the } \\
\text { material condition of Service Level } 1 \text { (see definitions of Service Levels in Attachment } 3 \text { of GL) } \\
\text { protective coatings inside the containment and to request information under } 10 \text { CFR 50.54(f) to } \\
\text { evaluate the addressees' programs for ensuring that Service Level } 1 \text { protective coatings inside } \\
\text { containment do not detach from their substrate during a DB LOCA and interfere with the operation of } \\
\text { the ECCS and the safety-related CSS. The NRC intends to use this information to assess whether } \\
\text { current regulatory requirements are being correctly implemented and whether they should be revised. } \\
\text { Discussion } \\
\text { Attachment } 2 \text { of the GL presents a tabulation of } 57 \text { operational events involving debris in the ECCS } \\
\text { recirculation flow paths. This tabulation includes the plant name, the report (e.g., LER, IN, NRC } \\
\text { Inspection Report) and a short description of the problem. } \\
\text { Related Generic Communications } \\
20 \text { Generic Communications listed related to ECCS and CSS sump and strainer blockage. }\end{array}$ & $\begin{array}{l}\text { ECCS and } \\
\text { CSS sump \& } \\
\text { drainage } \\
\text { blockage and } \\
\text { Containment } \\
\text { protective } \\
\text { coating }\end{array}$ \\
\hline GL & $\begin{array}{l}97-06 \\
(1997)\end{array}$ & $\begin{array}{l}\text { Degradatio } \\
\mathrm{n} \text { of Steam } \\
\text { Generator } \\
\text { Internals }\end{array}$ & $\begin{array}{l}\text { The U.S. Nuclear Regulatory Commission (NRC) is issuing this generic letter to (1) again alert } \\
\text { addressees to the previously communicated findings of damage to steam generator internals, namely, } \\
\text { tube support plates and tube bundle wrappers, at foreign PWR facilities; (2) alert addressees to recent } \\
\text { findings of damage to steam generator tube support plates at a U.S. PWR facility; (3) emphasize to } \\
\text { addressees the importance of performing comprehensive examinations of steam generator internals to }\end{array}$ & $\begin{array}{l}\text { Steam } \\
\text { Generators, } \\
\text { including } \\
\text { those } \\
\text { foreign } \\
\text { facilities }\end{array}$ \\
\hline
\end{tabular}




\begin{tabular}{|c|c|c|c|c|}
\hline Type & No. & Title & Topic & $\begin{array}{c}\text { SPC } \\
\text { Affected }\end{array}$ \\
\hline & & & $\begin{array}{l}\text { ensure steam generator tube structural integrity is maintained in accordance with the requirements of } \\
\text { Appendix B to } 10 \text { CFR Part 50; and (4) require all addressees to submit information that will enable } \\
\text { the NRC staff to verify whether addressees' steam generator internals comply with and conform to the } \\
\text { current licensing bases for their respective facilities. } \\
\text { Background: Foreign authorities have reported various steam generator tube support plate } \\
\text { damage mechanisms. The affected steam generators are similar, but not identical, to } \\
\text { Westinghouse Model } 51 \text { steam generators. As previously documented in IN } 96-09 \text { and IN 96- } \\
\text { 09, Supplement 1, one damage mechanism involved the wastage of the uppermost support } \\
\text { plate caused by the misapplication of a chemical cleaning process. A second damage } \\
\text { mechanism involved broken tube support plate ligaments at the uppermost, and sometimes } \\
\text { at the next lower, tube support plates. The support plate ligaments broke near a radial } \\
\text { seismic restraint and near an antirotation key; the damage apparently dates back to initial } \\
\text { startup of the affected plants. According to foreign authorities, the ligaments may have } \\
\text { broken because of excessive stress during the final thermal treatment of the monobloc steam } \\
\text { generators, which in turn was caused by inadequate clearance for differential thermal } \\
\text { expansion between the support plates, wrapper, and seismic restraints. } \\
\text { As previously documented in IN 96-09, Supplement } 1 \text {, a third damage mechanism involved } \\
\text { wastage not associated with chemical cleaning and affected tube support plates at various } \\
\text { elevations. This damage mechanism is active (progressive) and apparently involves a } \\
\text { corrosion or erosion-corrosion mechanism of undetermined origin. }\end{array}$ & \\
\hline GL & $\begin{array}{l}97-05 \\
(1997)\end{array}$ & $\begin{array}{l}\text { Steam } \\
\text { Generator } \\
\text { Tube } \\
\text { Inspection } \\
\text { Techniques }\end{array}$ & $\begin{array}{l}\text { The U.S. Nuclear Regulatory Commission (NRC) is issuing this generic letter to (1) emphasize to the } \\
\text { addressees the importance of performing steam generator tube inservice inspections using qualified } \\
\text { techniques in accordance with the requirements of Appendix B to } 10 \text { CFR Part 50, and (2) require } \\
\text { certain information from addressees to determine whether they are in compliance with the current } \\
\text { licensing basis for their respective facilities given their steam generator tube inservice inspection } \\
\text { practices. } \\
\text { Background: Licensees have traditionally relied upon eddy-current inspection techniques to assess the } \\
\text { condition of their steam generator tubes. Although the eddy- current method is a proven technique for } \\
\text { detecting tube degradation, the ability to depth size indications is possible only for specific modes of } \\
\text { degradation. Specifically, tube degradation from intergranular attack (IGA) and stress corrosion } \\
\text { cracking (SCC), major modes of steam generator tube degradation, are difficult to size with eddy- } \\
\text { current inspection techniques because of a number of complicating variables. In one recent instance, a }\end{array}$ & $\begin{array}{l}\text { Steam } \\
\text { Generators }\end{array}$ \\
\hline
\end{tabular}




\begin{tabular}{|c|c|c|c|c|}
\hline Туре & No. & Title & Topic & $\begin{array}{c}\text { SPC } \\
\text { Affected } \\
\end{array}$ \\
\hline & & & $\begin{array}{l}\text { licensee employed a technique to size the depths of IGA tube degradation based on tube specimens } \\
\text { removed from two plants. However, pulled tube data analyzed after the initial application of the } \\
\text { technique indicated that the method did not adequately estimate the true depth of the indications } \\
\text { consistent with the criteria established for qualifying the sizing technique. }\end{array}$ & \\
\hline GL & $\begin{array}{l}97-01 \\
(1997)\end{array}$ & $\begin{array}{l}\text { Degradatio } \\
\mathrm{n} \text { of } \\
\text { Control } \\
\text { Rod Drive } \\
\text { Mechanism } \\
\text { Nozzle and } \\
\text { Other } \\
\text { Vessel } \\
\text { Closure } \\
\text { Head } \\
\text { Penetration } \\
\mathrm{s}\end{array}$ & $\begin{array}{l}\text { The U.S. Nuclear Regulatory Commission (NRC) is issuing this generic letter to (1) request addressees } \\
\text { to describe their program for ensuring the timely inspection of PWR control rod drive mechanism } \\
\text { (CRDM) and other vessel closure head penetrations and (2) require that all addressees provide to the } \\
\text { NRC a written response to the requested information. The information requested is needed by the NRC } \\
\text { staff to verify compliance with } 10 \text { CFR } 50.55 \mathrm{a} \text { and } 10 \text { CFR Part } 50 \text {, Appendix A, GDC 14, and to } \\
\text { determine whether an augmented inspection program, pursuant to } 10 \text { CFR 50.55a(g)(6)(ii), is required. } \\
\text { Background: } \\
\text { Primary Water Stress Corrosion Cracking of Vessel Closure Head Penetrations - Most PWRs have } \\
\text { Alloy } 600 \text { CRDM nozzle and other vessel head closure penetrations (VHPs) that extend above the } \\
\text { reactor pressure vessel head. The stainless steel housing of the CRDM is screwed and seal-welded } \\
\text { onto the top of the nozzle penetration. The weld between the nozzle top and bottom pieces is a } \\
\text { dissimilar metal weld, which is also called a bimetallic weld. The nozzles protrude below the vessel } \\
\text { head, thus exposing the inside surface of the nozzles to reactor coolant. The CRDM nozzle and other } \\
\text { VHPs are basically the same for all PWRs worldwide, which use a U.S. design (except in Germany } \\
\text { and Russia). The areas of interest for potential cracking are the weld between the nozzle and reactor } \\
\text { vessel head, and the portion of the nozzle inside the reactor vessel head above the nozzle-to- vessel } \\
\text { weld. }\end{array}$ & $\begin{array}{l}\text { RPV }- \\
\text { closure head } \\
\text { penetrations }\end{array}$ \\
\hline $\begin{array}{l}\text { Bulletin } \\
\text { (BL) }\end{array}$ & 2004-01 & $\begin{array}{l}\text { Inspection } \\
\text { of Alloy } \\
82 / 182 / 600 \\
\text { Materials } \\
\text { Used in the } \\
\text { Fabrication } \\
\text { of } \\
\text { Pressurizer } \\
\text { Penetration } \\
\text { S and } \\
\text { Steam }\end{array}$ & $\begin{array}{l}\text { The U.S. Nuclear Regulatory Commission (NRC) is issuing this bulletin to: (1) advise PWR licensees } \\
\text { that current methods of inspecting Alloy } 82 / 182 / 600 \text { materials used in the fabrication of pressurizer } \\
\text { penetrations and steam space piping connections may need to be supplemented with additional } \\
\text { measures to detect and adequately characterize flaws due to primary water stress corrosion cracking } \\
\text { (PWSCC), } \\
\text { (2) request PWR addressees to provide the NRC with information related to the materials from which } \\
\text { the pressurizer penetrations and steam space piping connections at their facilities were fabricated, } \\
\text { (3) request PWR licensees to provide the NRC with information related to the inspections that have } \\
\text { been and those that will be performed to ensure that degradation of Alloy } 82 / 182 / 600 \text { materials used in } \\
\text { the fabrication of pressurizer penetrations and steam space piping connections will be identified, } \\
\text { adequately characterized, and repaired, and }\end{array}$ & $\begin{array}{l}\text { Pressurizer } \\
\text { and steam } \\
\text { space piping } \\
\text { connections } \\
\text { used in PWR } \\
\text { primary } \\
\text { coolant } \\
\text { systems }\end{array}$ \\
\hline
\end{tabular}




\begin{tabular}{|c|c|c|c|c|}
\hline Type & No. & Title & Topic & $\begin{array}{c}\text { SPC } \\
\text { Affected }\end{array}$ \\
\hline & & $\begin{array}{l}\text { Space } \\
\text { Piping } \\
\text { Connection } \\
\text { s at } \\
\text { Pressurized } \\
\text {-Water } \\
\text { Reactors }\end{array}$ & 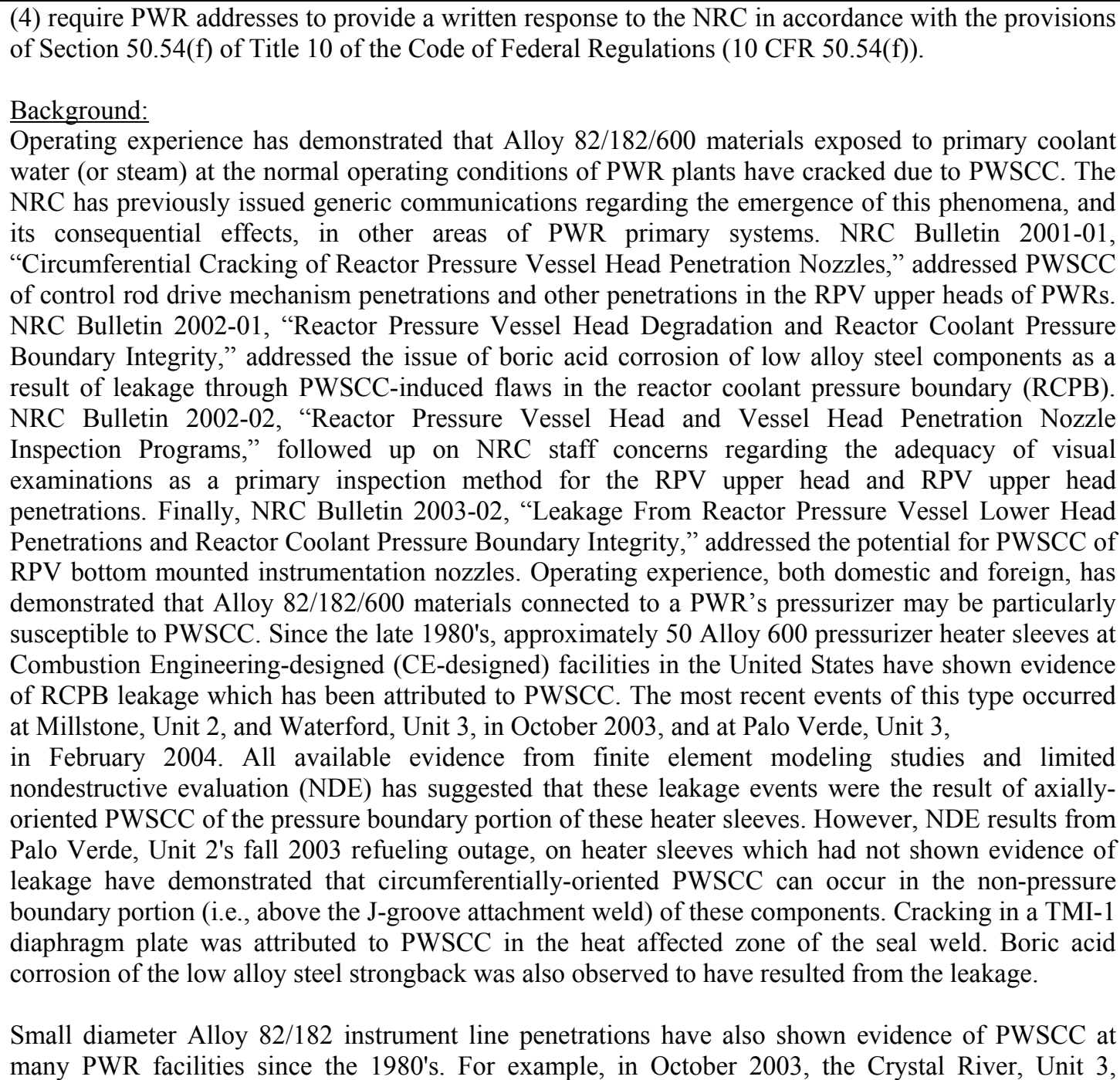 & \\
\hline
\end{tabular}




\begin{tabular}{|c|c|c|c|c|}
\hline Type & No. & Title & Topic & $\begin{array}{c}\text { SPC } \\
\text { Affected } \\
\end{array}$ \\
\hline & & & $\begin{array}{l}\text { licensee reported RCPB leakage from three pressurizer upper level instrument tap nozzles, which are } \\
\text { exposed to the steam space in the pressurizer. The leakage was attributed to PWSCC of Alloy } \\
\text { 82/182/600 material from which the connections were constructed. } \\
\text { Finally, inspection results from September } 2003 \text { at Tsuruga, Unit 2, in Japan are relevant with respect } \\
\text { to PWSCC in larger diameter, butt welded lines connected to the steam space of the pressurizer. } \\
\text { Evidence of boron deposits on the surface of a pressurizer relief valve nozzle (inside diameter } 130 \\
\text { mm, or approximately } 5 \text { inches) led to the discovery of five axially-oriented flaws in the nickel-based } \\
\text { alloy weld material used in the fabrication of the nozzle-to-safe end weld. Subsequent NDE performed } \\
\text { on a safety valve nozzle of similar diameter resulted in the discovery of two additional flaws in its } \\
\text { nozzle-to-safe end weld. Fractographic analysis of the flaw surfaces confirmed PWSCC as the } \\
\text { mechanism for flaw initiation and growth. This event at Tsuruga, Unit } 2 \text { was similar to an event at } \\
\text { Palisades in 1993 where leakage was observed and attributed to a circumferentially-oriented PWSCC } \\
\text { flaw in a line leading to the unit's power operated relief valves. } \\
\text { Related Generic Communications } \\
\text { - Bulletin 2003-02, "Leakage From Reactor Pressure Vessel Lower Head Penetrations and Reactor } \\
\text { Coolant Pressure Boundary Integrity," August 21, 2003 (ADAMS Accession No. ML032320153) } \\
\text { - Bulletin 2002-02, "Reactor Pressure Vessel Head and Vessel Head Penetration Nozzle } \\
\text { - Inspection Programs," August 9, 2002 (ADAMS Accession No. ML022200494) } \\
\text { - Bulletin 2002-01, "Reactor Pressure Vessel Head Degradation and Reactor Coolant Pressure } \\
\text { Boundary Integrity," March 18, 2002 (ADAMS Accession No. ML020770497) } \\
\text { - Bulletin 2001-01, "Circumferential Cracking of Reactor Pressure Vessel Head Penetration Nozzles," } \\
\text { August 3, 2001 (ADAMS Accession No. ML012080284) }\end{array}$ & \\
\hline $\mathrm{BL}$ & $2003-02$ & $\begin{array}{l}\text { Leakage } \\
\text { from } \\
\text { Reactor } \\
\text { Pressure } \\
\text { Vessel } \\
\text { Lower } \\
\text { Head } \\
\text { Penetration } \\
\text { S and } \\
\text { Reactor } \\
\text { Coolant }\end{array}$ & $\begin{array}{l}\text { The U.S. Nuclear Regulatory Commission (NRC) is issuing this bulletin to: } \\
\text { (1) advise PWR addressees that current methods of inspecting the RPV lower heads may need to be } \\
\text { supplemented with additional measures (e.g., bare-metal visual inspections) to detect reactor coolant } \\
\text { pressure boundary (RCPB) leakage, } \\
\text { (2) request PWR addressees to provide the NRC with information related to inspections that have been } \\
\text { or will be performed to verify the integrity of the RPV lower head penetrations, and } \\
\text { (3) require PWR addresses to provide a written response to the NRC in accordance with the provisions } \\
\text { of Section 50.54(f) of Title } 10 \text { of the Code of Federal Regulations (10 CFR 50.54(f)). } \\
\text { Discussion } \\
\text { The RPV and its head penetrations are an integral part of the RCPB, and their integrity is important to }\end{array}$ & $\begin{array}{l}\text { RPV - } \\
\text { closure head } \\
\text { penetrations }\end{array}$ \\
\hline
\end{tabular}




\begin{tabular}{|c|c|c|c|c|}
\hline Type & No. & Title & Topic & $\begin{array}{c}\text { SPC } \\
\text { Affected }\end{array}$ \\
\hline & & $\begin{array}{l}\text { Pressure } \\
\text { Boundary } \\
\text { Integrity }\end{array}$ & $\begin{array}{l}\text { the safe operation of the plant. The recent identification of cracking and leakage from two bottom } \\
\text { mounted instrumentation (BMI) penetrations at South Texas Project (STP) Unit } 1 \text { raises questions } \\
\text { about potential degradation mechanisms which may be active in this area. In addition, licensee } \\
\text { responses to the Bulletin 2002-01 followup RAIs raised questions about the adequacy of inspections } \\
\text { performed by licensees to detect leakage from RPV lower head penetrations. } \\
\text { Related Generic Communications } \\
\text { - Regulatory Issue Summary 2003-13, "NRC Review of Responses to Bulletin 2002-01, 'Reactor } \\
\text { Pressure Vessel Head Degradation and Reactor Coolant Pressure Boundary Integrity,'July 29, } 2003 \\
\text { (ADAMS Accession No. ML032100653) } \\
\text { - Information Notice 2003-11 "Leakage Found on Bottom-Mounted Instrumentation Nozzles," August } \\
\text { 13, } 2003 \text { (ADAMS Accession No. ML032250135) } \\
\text { - Bulletin 2002-02, "Reactor Pressure Vessel Head and Vessel Head Penetration Nozzle Inspection } \\
\text { Programs," August 9, 2002 (ADAMS Accession No. ML022200494) } \\
\text { - Bulletin 2002-01, "Reactor Pressure Vessel Head Degradation and Reactor Coolant Pressure } \\
\text { Boundary Integrity," March 18, 2002 (ADAMS Accession No. ML020770497) } \\
\text { - Generic Letter 88-05, "Boric Acid Corrosion of Carbon Steel Reactor Pressure Boundary } \\
\text { Components in PWR Plants," March 17, 1988 (ADAMS Accession No. ML031130424) }\end{array}$ & \\
\hline $\mathrm{BL}$ & $2003-01$ & $\begin{array}{l}\text { Potential } \\
\text { Impact of } \\
\text { Debris } \\
\text { Blockage } \\
\text { on } \\
\text { Emergency } \\
\text { Sump at } \\
\text { Recirculati } \\
\text { on Pressurized } \\
\text {-Water } \\
\text { Reactors }\end{array}$ & $\begin{array}{l}\text { The U.S. Nuclear Regulatory Commission (NRC) is issuing this bulletin to: } \\
\text { (1) Inform addressees of the results of NRC-sponsored research identifying the potential susceptibility } \\
\text { of pressurized-water reactor (PWR) recirculation sump screens to debris blockage in the event of a } \\
\text { high-energy line break (HELB) requiring recirculation operation of the emergency core cooling system } \\
\text { (ECCS) or containment spray system (CSS). } \\
\text { (2) Inform addressees of the potential for additional adverse effects due to debris blockage of } \\
\text { flowpaths necessary for ECCS and CSS recirculation and containment drainage. } \\
\text { (3) Request that, in light of these potentially adverse effects, addressees confirm their compliance with } \\
10 \text { CFR 50.46(b)(5) and other existing applicable regulatory requirements, or describe any } \\
\text { compensatory measures implemented to reduce the potential risk due to post-accident debris blockage } \\
\text { as evaluations to determine compliance proceed. } \\
\text { (4) Require addressees to provide the NRC a written response in accordance with } 10 \text { CFR 50.54(f). } \\
\text { Discussion } \\
\text { See discussion in GL 2004-02 above. } \\
\text { Related Generic Communications }\end{array}$ & Strainers \\
\hline
\end{tabular}




\begin{tabular}{|c|c|c|c|c|}
\hline Type & No. & Title & Topic & $\begin{array}{c}\text { SPC } \\
\text { Affected }\end{array}$ \\
\hline & & & See list in GL 2004-02 above. & \\
\hline $\mathrm{BL}$ & $2002-02$ & $\begin{array}{l}\text { Reactor } \\
\text { Pressure } \\
\text { Vessel } \\
\text { Head and } \\
\text { Vessel } \\
\text { Head } \\
\text { Penetration } \\
\text { Nozzle } \\
\text { Inspection } \\
\text { Programs }\end{array}$ & $\begin{array}{l}\text { The U.S. Nuclear Regulatory Commission (NRC) is issuing this bulletin to: } \\
\text { (1) Advise pressurized-water reactor (PWR) addressees that visual examinations, as a primary } \\
\text { inspection method for the reactor pressure vessel (RPV) head and vessel head penetration (VHP) } \\
\text { nozzles, may need to be supplemented with additional measures (e.g., volumetric and surface } \\
\text { examinations) to demonstrate compliance with applicable regulations. } \\
\text { (2) Advise PWR addressees that inspection methods and frequencies to demonstrate } \\
\text { compliance with applicable regulations should be demonstrated to be reliable and effective. } \\
\text { (3) Request information from all PWR addressees concerning their RPV head and VHP nozzle } \\
\text { inspection programs to ensure compliance with applicable regulatory requirements. } \\
\text { (4) Require all PWR addressees to provide written responses to this bulletin related to their inspection } \\
\text { program plans. } \\
\text { Discussion } \\
\text { As a result of the circumferential cracking of VHP nozzles at Oconee Nuclear Station } 3 \text { and other } \\
\text { PWR facilities, the RPV head material degradation at Davis-Besse, and the staff's review of responses } \\
\text { to NRC Bulletins } 2001-01 \text { and } 2002-01 \text {, the NRC staff has a number of concerns about the inspection } \\
\text { requirements and programs for RPV head and VHP nozzles. Based on the experience and information } \\
\text { currently available concerning cracking and degradation, it may } \\
\text { be necessary for inspection programs that rely on visual examinations to be supplemented with } \\
\text { additional measures (e.g., volumetric and surface examinations) to demonstrate compliance with } \\
\text { applicable regulations. } \\
\text { The NRC has developed Web pages to keep the public informed of generic activities related to Alloy } \\
600 \text { cracking and RPV head degradation: } \\
\text { http://www.nrc.gov/reactors/operating/ops-experience/alloy600.html } \\
\text { http://www.nrc.gov/reactors/operating/ops-experience/vessel-head-degradation.html } \\
\text { See GL 2003-02 above. }\end{array}$ & $\begin{array}{l}\text { RPV head } \\
\text { and vessel } \\
\text { head } \\
\text { penetrations }\end{array}$ \\
\hline $\mathrm{BL}$ & $2002-01$ & $\begin{array}{l}\text { Reactor } \\
\text { Pressure } \\
\text { Vessel } \\
\text { Head }\end{array}$ & $\begin{array}{l}\text { The U.S. Nuclear Regulatory Commission (NRC) is issuing this bulletin to require pressurized-water } \\
\text { reactor (PWR) addressees to submit: } \\
\text { (1) information related to the integrity of the reactor coolant pressure boundary including the reactor }\end{array}$ & $\begin{array}{l}\text { RPV and } \\
\text { reactor } \\
\text { coolant } \\
\text { pressure }\end{array}$ \\
\hline
\end{tabular}




\begin{tabular}{|c|c|c|c|c|}
\hline Type & No. & Title & Topic & $\begin{array}{c}\text { SPC } \\
\text { Affected }\end{array}$ \\
\hline & & $\begin{array}{l}\text { Degradatio } \\
\mathrm{n} \quad \text { and } \\
\text { Reactor } \\
\text { Coolant } \\
\text { Pressure } \\
\text { Boundary } \\
\text { Integrity }\end{array}$ & 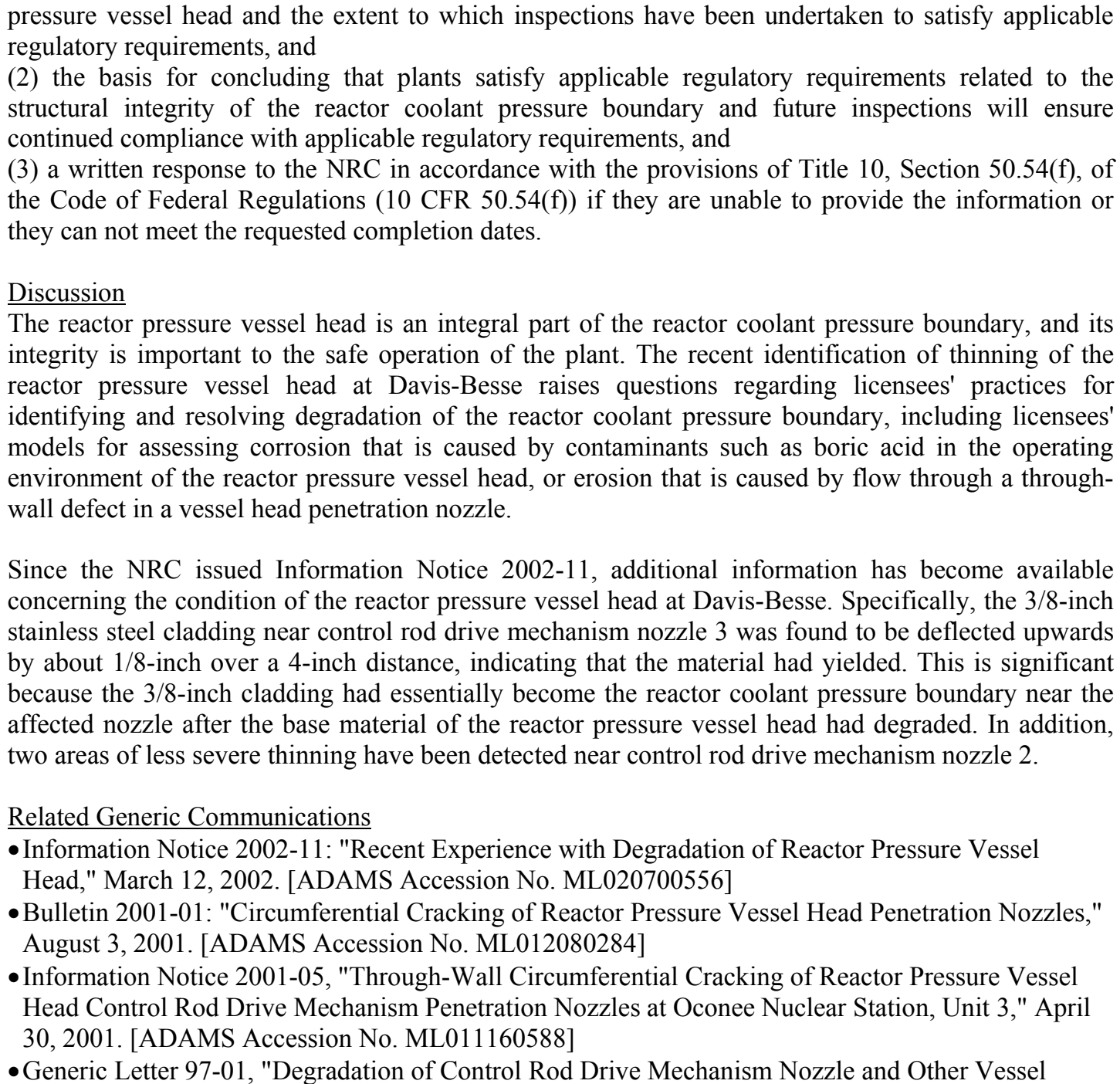 & boundary \\
\hline
\end{tabular}




\begin{tabular}{|c|c|c|c|c|}
\hline Туре & No. & Title & Topic & $\begin{array}{c}\text { SPC } \\
\text { Affected }\end{array}$ \\
\hline & & & 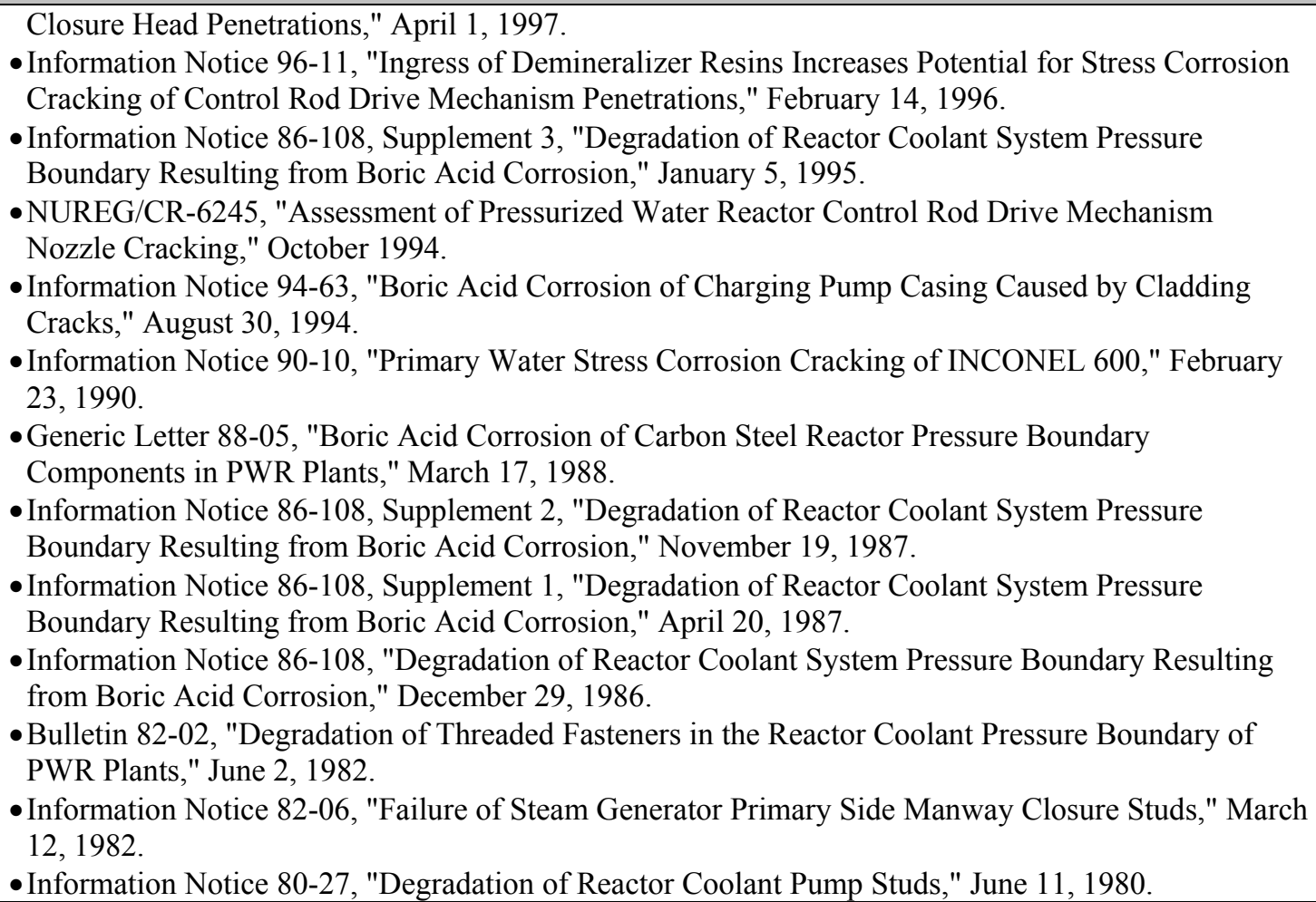 & \\
\hline $\mathrm{BL}$ & $2001-01$ & $\begin{array}{l}\text { Circumfere } \\
\text { ntial } \\
\text { Cracking of } \\
\text { Reactor } \\
\text { Pressure } \\
\text { Vessel } \\
\text { Head } \\
\text { Penetration } \\
\text { Nozzles }\end{array}$ & $\begin{array}{l}\text { The U.S. Nuclear Regulatory Commission (NRC) is issuing this bulletin to: } \\
\text { (1) request that addressees provide information related to the structural integrity of the reactor pressure } \\
\text { vessel head penetration (VHP) nozzles for their respective facilities, including the extent of VHP } \\
\text { nozzle leakage and cracking that has been found to date, the inspections and repairs that have been } \\
\text { undertaken to satisfy applicable regulatory requirements, and the basis for concluding that their plans } \\
\text { for future inspections will ensure compliance with applicable regulatory requirements, and } \\
\text { (2) require that all addressees provide to the NRC a written response in accordance with the provisions } \\
\text { of } 10 \text { CFR 50.54(f). } \\
\text { Discussion }\end{array}$ & $\begin{array}{l}\text { RPV head } \\
\text { penetrations }\end{array}$ \\
\hline
\end{tabular}




\begin{tabular}{|c|c|c|c|c|}
\hline Type & No. & Title & Topic & $\begin{array}{c}\text { SPC } \\
\text { Affected }\end{array}$ \\
\hline & & & $\begin{array}{l}\text { The recent discoveries of cracked and leaking Alloy } 600 \text { VHP nozzles, including control rod drive } \\
\text { mechanism (CRDM) and thermocouple nozzles, at four pressurized water reactors (PWRs) have raised } \\
\text { concerns about the structural integrity of VHP nozzles throughout the PWR industry. Nozzle cracking } \\
\text { at Oconee Nuclear Station Unit } 1 \text { (ONS1) in November } 2000 \text { and Arkansas Nuclear One Unit } 1 \\
\text { (ANO1) in February } 2001 \text { was limited to axial cracking, an occurrence deemed to be of limited safety } \\
\text { concern in the NRC staff's generic safety evaluation on the cracking of VHP nozzles, dated November } \\
\text { 19, 1993. However, the discovery of circumferential cracking at Oconee Nuclear Station Unit } 3 \\
\text { (ONS3) in February } 2001 \text { and Oconee Nuclear Station Unit } 2 \text { (ONS2) in April } 2001 \text { - particularly the } \\
\text { large circumferential cracking identified in two CRDM nozzles at ONS3 - has raised concerns about } \\
\text { the potential safety implications and prevalence of cracking in VHP nozzles in PWRs. } \\
\text { Related Generic Communications } \\
\text { - Information Notice 2001-05, "Through-Wall Circumferential Cracking of Reactor Pressure Vessel } \\
\text { Head Control Rod Drive Mechanism Penetration Nozzles at Oconee Nuclear Station, Unit 3," April } \\
\text { 30, 2001. [ADAMS Accession No. ML011160588] } \\
\text { - Generic Letter 97-01, "Degradation of Control Rod Drive Mechanism Nozzle and Other Vessel } \\
\text { Closure Head Penetrations," April 1, 1997. } \\
\text { - Information Notice 96-11, "Ingress of Demineralizer Resins Increases Potential for Stress Corrosion } \\
\text { Cracking of Control Rod Drive Mechanism Penetrations," February 14, 1996. } \\
\text { - Information Notice 90-10, "Primary Water Stress Corrosion Cracking of INCONEL 600," February } \\
\text { 23, 1990. } \\
\text { - Generic Letter 88-05, "Boric Acid Corrosion of Carbon Steel Reactor Pressure Boundary } \\
\text { Components in PWR Plants," March 17, 1988. } \\
\text { - NUREG/CR-6245, "Assessment of Pressurized Water Reactor Control Rod Drive Mechanism } \\
\text { Nozzle Cracking," October 1994. }\end{array}$ & \\
\hline $\begin{array}{l}\text { Informati } \\
\text { on } \\
\text { Notices } \\
\text { (IN) }\end{array}$ & $2007-37$ & $\begin{array}{l}\text { Buildup of } \\
\text { Deposits in } \\
\text { Steam } \\
\text { Generator }\end{array}$ & $\begin{array}{l}\text { The U.S. Nuclear Regulatory Commission (NRC) is issuing this information notice (IN) to alert } \\
\text { addressees to the potential for deposits to accumulate in their steam generators and potentially affect } \\
\text { steam generator performance and tube integrity. The NRC expects that recipients of this IN will } \\
\text { review the information for applicability to their facilities and consider taking actions, as appropriate, to } \\
\text { avoid similar problems. However, suggestions contained in this IN are not NRC } \\
\text { requirements; therefore, no specific action or written response is required. } \\
\text { Discussion } \\
\text { Corrosion products can accumulate in the secondary side of the steam generator as a result of the } \\
\text { gradual erosion and corrosion of secondary side components in a pressurized water reactor. This }\end{array}$ & $\begin{array}{l}\text { Steam } \\
\text { Generators }\end{array}$ \\
\hline
\end{tabular}




\begin{tabular}{|c|c|c|c|c|}
\hline Type & No. & Title & Topic & $\begin{array}{c}\text { SPC } \\
\text { Affected }\end{array}$ \\
\hline & & & $\begin{array}{l}\text { accumulation of corrosion products results in the buildup of deposits on the tubes, tubesheets, and } \\
\text { other secondary side steam generator structures (including the holes through which the tubes pass). } \\
\text { Harmful contaminants can concentrate in these deposits and result in corrosion of the steam generator } \\
\text { tubes. In addition, these deposits can affect the thermal performance of the steam generator (i.e., the } \\
\text { ability to transfer heat from the primary-to-secondary side of the steam generator) and the thermal } \\
\text { hydraulic characteristics of the steam generator (by changing the flow patterns within the steam } \\
\text { generator). } \\
\text { Between } 2004 \text { and 2006, three primary-to-secondary leaks occurred at the Cruas Nuclear Plant, a } \\
\text { multi-unit site in France. The last primary-to-secondary leak occurred at Cruas Unit } 4 \text { in February } \\
2006 \text { (Autorite de Surrete Nucléaire } 2006 \text { Annual Report, http://annual-report.asn.fr/PDF/nuclear- } \\
\text { power-plants-EDF.pdf), and it was detected through the use of nitrogen-16 radiation monitors. The } \\
\text { leak rate increased from very low levels to approximately } 3 \text { gallons per minute [600 liters per hour] in } \\
12 \text { minutes. } \\
\text { In the early } 1990 \text { s, steam generator water level oscillations were observed at Surry Power Units } 1 \text { and } \\
2 \text {, near Newport News, Virginia. Due to the severity of these water level oscillations, the units } \\
\text { operated at reduced power levels for varying periods of time. The cause of the steam generator water } \\
\text { level oscillations was severe deposit buildup in the TSP quatrefoil-shaped holes. The licensee } \\
\text { corrected the problem by performing chemical cleaning on all steam } \\
\text { generators at both units to reduce the extent of deposits. }\end{array}$ & \\
\hline IN & $2007-21$ & $\begin{array}{l}\text { Pipe Wear } \\
\text { Due to } \\
\text { Interaction } \\
\text { of Flow- } \\
\text { induced } \\
\text { Vibration } \\
\text { and } \\
\text { Reflective } \\
\text { Metal } \\
\text { Insulation }\end{array}$ & $\begin{array}{l}\text { The U.S. Nuclear Regulatory Commission (NRC) is issuing this information notice (IN) to alert } \\
\text { addressees that a licensee identified significant wear marks on the outside wall of chemical volume } \\
\text { control system (CVCS) stainless steel piping, which was subject to flow-induced vibration conditions. } \\
\text { The licensee determined that the wear marks were caused by the interaction between the piping base } \\
\text { metal and the properly installed reflective metal insulation (RMI). The NRC expects that addressees } \\
\text { will review the information for applicability to their facilities and consider actions, as appropriate, to } \\
\text { identify and address similar problems. However, suggestions contained in this IN are not NRC } \\
\text { requirements; therefore, no specific action or written response is required. } \\
\text { During a Catawba Unit } 1 \text { refueling outage conducted in the fall of } 2006 \text {, the licensee identified } \\
\text { multiple wear marks on CVCS field-run stainless steel piping (see Enclosure, Figure 1) that was } \\
\text { downstream of the CVCS letdown orifices. The licensee determined that these marks were a result of } \\
\text { abrasive wear between the stainless steel RMI end caps and the stainless steel piping. This abrasive } \\
\text { wear was most probably caused by the known flow-induced vibration downstream of the letdown }\end{array}$ & Piping \\
\hline
\end{tabular}




\begin{tabular}{|c|c|c|c|c|}
\hline Type & No. & Title & Topic & $\begin{array}{c}\text { SPC } \\
\text { Affected } \\
\end{array}$ \\
\hline & & & $\begin{array}{l}\text { orifices combined with end cap to piping interaction. RMI is assembled by clipping short segments of } \\
\text { insulation together. End caps are found at the intersection of each insulation segment, and these end } \\
\text { caps are perpendicular to the pipe wall (see Enclosure, Figure 2). The licensee confirmed that the RMI } \\
\text { end caps had been installed properly in accordance with plant procedures and vendor instructions. } \\
\text { None of the wear marks around the } \\
\text { piping were continuous for } 360 \text { degrees and most extended less than } 180 \text { degrees of the circumference. } \\
\text { The deepest wear mark was one thirty-seconds of an inch. All of the CVCS piping with identified wear } \\
\text { marks was located inside containment. }\end{array}$ & \\
\hline IN & $2006-27$ & $\begin{array}{l}\text { Circumfere } \\
\text { ntial } \\
\text { Cracking in } \\
\text { the } \\
\text { Stainless } \\
\text { Steel } \\
\text { Pressurizer } \\
\text { Heater } \\
\text { Sleeves of } \\
\text { Pressurized } \\
\text { Water } \\
\text { Reactors }\end{array}$ & $\begin{array}{l}\text { The U.S. Nuclear Regulatory Commission (NRC) is issuing this information notice (IN) to describe a } \\
\text { recent experience in which a licensee attributed a circumferentially-oriented crack to intergranular } \\
\text { stress corrosion cracking (IGSCC) in a stainless steel pressurizer heater sleeve in a PWR reactor } \\
\text { coolant environment. The NRC expects that addressees will review the information for applicability to } \\
\text { their facilities and consider actions, as appropriate, to identify and address similar problems. However, } \\
\text { suggestions contained in this IN are not NRC requirements; therefore, no specific action or written } \\
\text { response is required. } \\
\text { Discussion } \\
\text { During the spring } 2006 \text { outage at Braidwood, Unit } 1 \text {, Exelon Generation Company (the licensee) found } \\
\text { boron deposits in the pressurizer surge line area during insulation removal. The licensee determined } \\
\text { that the leakage originated from the number } 52 \text { pressurizer heater (heater number } 52 \text { ) at the upper weld } \\
\text { between the pressure tube and heater coupling. The licensee based this determination on deposit } \\
\text { patterns, deposit chemical analysis, and rouging } \\
\text { (i.e., rust) found in the convection cover insulation sleeve for heater number } 52 \text {. Rouging could be } \\
\text { caused by steam impingement on the stainless steel material. The licensee visually inspected all } 78 \\
\text { pressurizer heaters to determine the extent of the condition and determined that heater number } 52 \text { was } \\
\text { the only source of boric acid leakage from the pressurizer. The licensee removed leaking coupling for } \\
\text { heater number } 52 \text { from the system and plugged the tube. The licensee shipped the coupling to a testing } \\
\text { facility to determine the cause of the } \\
\text { failure. The results of the laboratory examinations to date suggest that the observed cracking in the } \\
\text { sleeve occurred due to circumferentially-oriented IGSCC in the heat affected zone. }\end{array}$ & $\begin{array}{l}\text { Pressurizer } \\
\text { heater } \\
\text { sleeves }\end{array}$ \\
\hline IN & $2006-17$ & $\begin{array}{l}\text { Recent } \\
\text { Operating } \\
\text { Experience } \\
\text { of Service } \\
\text { Water }\end{array}$ & $\begin{array}{l}\text { The U.S. Nuclear Regulatory Commission (NRC) is issuing this information notice (IN) to inform } \\
\text { addressees of operating experience within the past few years affecting the operability of the service } \\
\text { water system at several nuclear power plants. The NRC expects that recipients will review the } \\
\text { information for applicability to their facilities and consider actions, as appropriate, to avoid similar } \\
\text { problems. However, suggestions contained in this IN are not NRC requirements; therefore, no specific }\end{array}$ & $\begin{array}{l}\text { Piping - } \\
\text { service water } \\
\text { systems - } \\
\text { plugging/foul } \\
\text { ing }\end{array}$ \\
\hline
\end{tabular}




\begin{tabular}{|c|c|c|c|c|}
\hline Type & No. & Title & Topic & $\begin{array}{c}\text { SPC } \\
\text { Affected }\end{array}$ \\
\hline & & $\begin{array}{l}\text { Systems } \\
\text { Due to } \\
\text { External } \\
\text { Conditions }\end{array}$ & $\begin{array}{l}\text { action or written response is required. } \\
\text { Discussion } \\
\text { During } 2004 \text { through } 2005,15 \text { events occurred related to blockages in service water systems. These } \\
\text { events were primarily self-revealing. The various blocking agents included silt, sand, small rocks, } \\
\text { grass or weeds, frazil ice, and small aquatic fauna, such as fish. All these events were of low safety } \\
\text { significance but illustrate the susceptibility of the safety-significant service water system. For instance, } \\
\text { in September } 2005, \text { NRC inspectors identified a condition at Fort } \\
\text { Calhoun that allowed small rocks to regularly enter the raw water system, contribute to tripping of a } \\
\text { pump and strainer motors, and interfere with traveling screen operation (NRC Inspection Report } 50- \\
285 / 2005-11 \text {, Agencywide Documents Access and Management System (ADAMS) Accession No. } \\
\text { ML052920543). In June } 2005 \text {, NRC inspectors found a portion of a service } \\
\text { water accumulator outlet line at Salem to be nearly full of silt (NRC Inspection } \\
\text { Report 50-272/2005-03, ADAMS Accession No. ML052090344). Other occurrences are also } \\
\text { described for Watts Bar and Cooper Nuclear nuclear plants. }\end{array}$ & \\
\hline IN & 2006-08 & $\begin{array}{l}\text { Secondary } \\
\text { Piping } \\
\text { Rupture at } \\
\text { the } \\
\text { Mihama } \\
\text { Power } \\
\text { Station in } \\
\text { Japan }\end{array}$ & $\begin{array}{l}\text { The U.S. Nuclear Regulatory Commission (NRC) is issuing this information notice (IN) involving } \\
\text { foreign operating experience to alert addressees of the root causes and lessons learned from a } \\
\text { secondary piping rupture at the Mihama Power Station, Unit } 3 \text { (Mihama } 3 \text { ) in Japan. It is expected that } \\
\text { recipients will review the information for applicability to their facilities and consider actions, as } \\
\text { appropriate, to avoid similar problems. However, suggestions contained in this } \\
\text { information notice are not NRC requirements; therefore, no specific action or written response is } \\
\text { required. } \\
\text { Discussion } \\
\text { The Mihama 3, is an } 826 \text { Megawatts electric, 3-loop Westinghouse type pressurized-water reactor } \\
\text { (PWR) owned by Kansai Electric Power Company, Inc., and licensed by the Japanese government. } \\
\text { This unit has been in service since } 1976 \text {. On August } 9 \text {, } 2004 \text {, a fire alarm annunciated in the central } \\
\text { control room at Mihama } 3 \text {. Upon investigation, operators discovered the area covered by the alarm } \\
\text { was filled with steam. Suspecting that steam or high temperature water was leaking from the } \\
\text { secondary piping, the operators began an emergency load reduction. While they were doing this, the } \\
\text { reactor tripped automatically based on the steam flow from the } 3 \text { A steam generator exceeding the } \\
\text { feedwater flow to that steam generator. The rupture occurred in a } 55.9 \text { centimeter (cm) ( } 22 \text { inch) } \\
\text { outside diameter pipe in the 'A' loop condensate system between the fourth feedwater heater and the } \\
\text { deaerator, downstream of an orifice for measuring single-phase water flow. At the time of the } \\
\text { secondary piping rupture, } 105 \text { workers were preparing for the periodic inspections to commence. The }\end{array}$ & $\begin{array}{l}\text { Secondary } \\
\text { piping } \\
\text { rupture }\end{array}$ \\
\hline
\end{tabular}




\begin{tabular}{|c|c|c|c|c|}
\hline Type & No. & Title & Topic & $\begin{array}{c}\text { SPC } \\
\text { Affected } \\
\end{array}$ \\
\hline & & & $\begin{array}{l}\text { accident resulted in five deaths and six injuries. } \\
\text { A microscopic inspection of the inside surface of the ruptured pipe revealed a fish scale-like pattern } \\
\text { over almost the entire inner surface of the pipe downstream of the orifice, except at the bottom of the } \\
\text { pipe. The thickness along the bottom of the pipe was found to be the nominal wall thickness. The } \\
\text { inside surface of the bottom of the pipe was covered with a thick surface film. These conditions are } \\
\text { characteristic of flow-accelerated corrosion (FAC). }\end{array}$ & \\
\hline IN & $2006-01$ & $\begin{array}{l}\text { Torus } \\
\text { Cracking in } \\
\text { a BWR } \\
\text { Mark I } \\
\text { Containme } \\
\text { nt }\end{array}$ & $\begin{array}{l}\text { The U.S. Nuclear Regulatory Commission (NRC) is issuing this information notice (IN) to inform the } \\
\text { owners of BWR Mark I containments about the occurrence and potential causes of the through-wall } \\
\text { cracking of a torus in a BWR Mark I containment. Recipients are expected to review the information } \\
\text { for applicability to their facilities and consider appropriate actions to avoid similar problems. } \\
\text { However, the measures suggested in this IN are not NRC requirements; therefore, no specific action or } \\
\text { written response is required. } \\
\text { Discussion } \\
\text { On June 27, 2005, with the plant operating at } 100 \text {-percent power during a licensee inspection of } \\
\text { reactor core isolation cooling system torus suction piping, James A. FitzPatrick Nuclear Power Plant } \\
\text { (FitzPatrick) personnel discovered a torus leak near a torus support. The plant's torus is a large } \\
\text { doughnut-shaped steel structure that is partially filled with water and designed to act as a pressure } \\
\text { suppression chamber. The leak was located about } 5 \text { feet below the waterline and just below the high- } \\
\text { pressure coolant injection (HPCI) turbine exhaust pipe. The leak was characterized as a slight seepage } \\
\text { with streaking and a small puddle below the leak. Subsequent nondestructive examination determined } \\
\text { that the leakage was from a small through-wall torus crack which was x-shaped with an approximate } \\
\text { 4.6 inch maximum length. } \\
\text { The FitzPatrick licensee performed a root cause investigation of the event, and after eliminating a } \\
\text { number of possible causes, the licensee concluded that the most likely cause for the initiation and } \\
\text { propagation of the crack was the hydrodynamic loads of the turbine exhaust pipe during HPCI } \\
\text { operation coupled with the highly restrained condition of the torus shell at the torus column support. } \\
\text { The licensee concluded that the crack was initiated by cyclic loading due to condensation oscillation } \\
\text { during HPCI operation. }\end{array}$ & $\begin{array}{l}\text { Steel } \\
\text { Containment } \\
\text { - Torus }\end{array}$ \\
\hline IN & $2004-21$ & $\begin{array}{l}\text { Additional } \\
\text { Adverse } \\
\text { Effect of }\end{array}$ & $\begin{array}{l}\text { The U.S. Nuclear Regulatory Commission (NRC) is issuing this information notice (IN) to inform } \\
\text { addressees of potential adverse effects of boric acid leakage that may not have been previously } \\
\text { considered and to reemphasize concerns regarding boric acid accumulations on reactor plant }\end{array}$ & $\begin{array}{l}\text { Potential } \\
\text { Boric Acid } \\
\text { Corrosion on }\end{array}$ \\
\hline
\end{tabular}




\begin{tabular}{|c|c|c|c|c|}
\hline Туре & No. & Title & Topic & $\begin{array}{c}\text { SPC } \\
\text { Affected }\end{array}$ \\
\hline & & $\begin{array}{l}\text { Boric Acid } \\
\text { Leakage: } \\
\text { Potential } \\
\text { Impact on } \\
\text { Post- } \\
\text { Accident } \\
\text { Coolant pH }\end{array}$ & $\begin{array}{l}\text { equipment inside containment. The primary concern regarding boric acid leakage is corrosion of } \\
\text { ferritic steel components. However, if boric acid deposits of sufficient magnitude are present in } \\
\text { containment, dissolution of these deposits may also affect the pH of the reactor coolant in the } \\
\text { containment sump. The NRC anticipates that recipients will review the information for applicability to } \\
\text { their facilities and consider appropriate actions. However, suggestions contained in this IN do not } \\
\text { constitute NRC requirements; therefore, no specific action or written response is required. } \\
\text { Discussion } \\
\text { During refueling outages throughout the 1990s, personnel at the Davis-Besse nuclear power plant } \\
\text { performed visual inspections of the reactor pressure vessel (RPV) head surface that was accessible } \\
\text { through the service structure weep holes. Visual inspections performed below the RPV head insulation } \\
\text { found some accumulation of boric acid deposits on the RPV head. The boric acid buildup was due to } \\
\text { leaking control rod drive mechanism flanges and reactor coolant pressure boundary leakage. Many } \\
\text { areas of the RPV head were not visible because of persistent boric acid deposits that the licensee did } \\
\text { not clean. In addition to the significant buildup of boric acid on the reactor pressure vessel head, a } \\
\text { substantial amount of boric acid built up inside the containment at Davis-Besse. }\end{array}$ & $\begin{array}{l}\text { Various } \\
\text { Equipment }\end{array}$ \\
\hline IN & 2004-11 & $\begin{array}{l}\text { Cracking in } \\
\text { Pressurizer } \\
\text { Safety and } \\
\text { Relief } \\
\text { Nozzles } \\
\text { and in } \\
\text { Surge Line } \\
\text { Nozzle }\end{array}$ & $\begin{array}{l}\text { The U.S. Nuclear Regulatory Commission (NRC) is issuing this information notice (IN) to alert } \\
\text { addressees to cracking and leakage indications found on pressurizer safety and relief nozzles and in a } \\
\text { surge line nozzle-to-safe end weld. It is expected that the recipients of this notice will review the } \\
\text { information for applicability to their facilities and consider actions, as appropriate, to avoid similar } \\
\text { problems. However, suggestions contained in this information notice are not NRC requirements; } \\
\text { therefore, no specific action or written response is required. } \\
\text { Discussion } \\
\text { During an annual inspection in September of } 2003 \text {, cracking and leakage were discovered on } \\
\text { pressurizer safety and relief nozzles in Tsuruga Power Plant, Unit } 2 \text { (Tsuruga } 2) \text {, in Japan. Tsuruga } 2 \text { is } \\
\text { a four-loop pressurized water reactor (PWR) unit (similar to the PWRs in the U.S). Tsuruga } 2 \text {, which } \\
\text { started commercial operation in February } 1987 \text {, was designed and fabricated by Mitsubishi Heavy } \\
\text { Industries. Full power for Tsuruga } 2 \text { is } 1160 \mathrm{MWe} \text {. At } 100^{\circ} \text { power, the average primary coolant } \\
\text { temperature is } 289^{\circ} \mathrm{C}\left(552^{\circ} \mathrm{F}\right) \text { in the cold leg and } 322^{\circ} \mathrm{C}\left(612^{\circ} \mathrm{F}\right) \text { in the hot leg. } \\
\text { During a refueling outage in October } 2003 \text {, an indication was detected in a surge line nozzle-to-safe } \\
\text { end dissimilar metal weld at Three Mile Island, Unit } 1 \text { (TMI-1). TMI- } 1 \text { is a Babcock and Wilcox } \\
\text { pressurized water reactor which started commercial operation in September } 1974 \text {. }\end{array}$ & $\begin{array}{l}\text { Pressurizer } \\
\text { nozzles }\end{array}$ \\
\hline IN & $2004-09$ & Corrosion & The U.S. Nuclear Regulatory Commission (NRC) is issuing this information notice to alert addressees & Steel \\
\hline
\end{tabular}




\begin{tabular}{|c|c|c|c|c|}
\hline Туре & No. & Title & Topic & $\begin{array}{c}\text { SPC } \\
\text { Affected }\end{array}$ \\
\hline & & $\begin{array}{l}\text { of Steel } \\
\text { Containme } \\
\text { nt and } \\
\text { Containme } \\
\text { nt Liner }\end{array}$ & 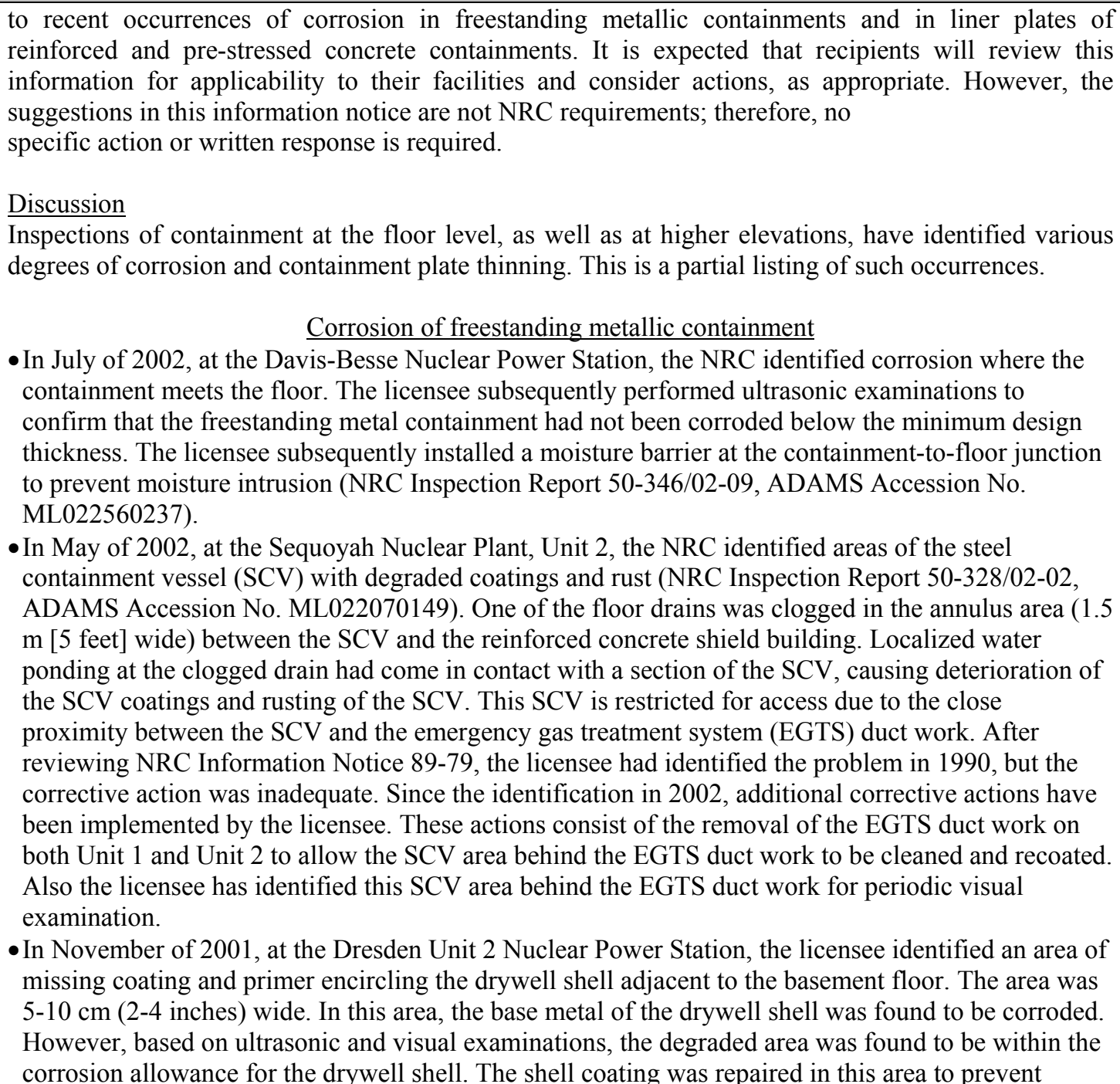 & $\begin{array}{l}\text { Containment } \\
\& \text { Liner of } \\
\text { Concrete } \\
\text { Containment }\end{array}$ \\
\hline
\end{tabular}




\begin{tabular}{|c|c|c|c|c|}
\hline Туре & No. & Title & Topic & $\begin{array}{c}\text { SPC } \\
\text { Affected }\end{array}$ \\
\hline & & & 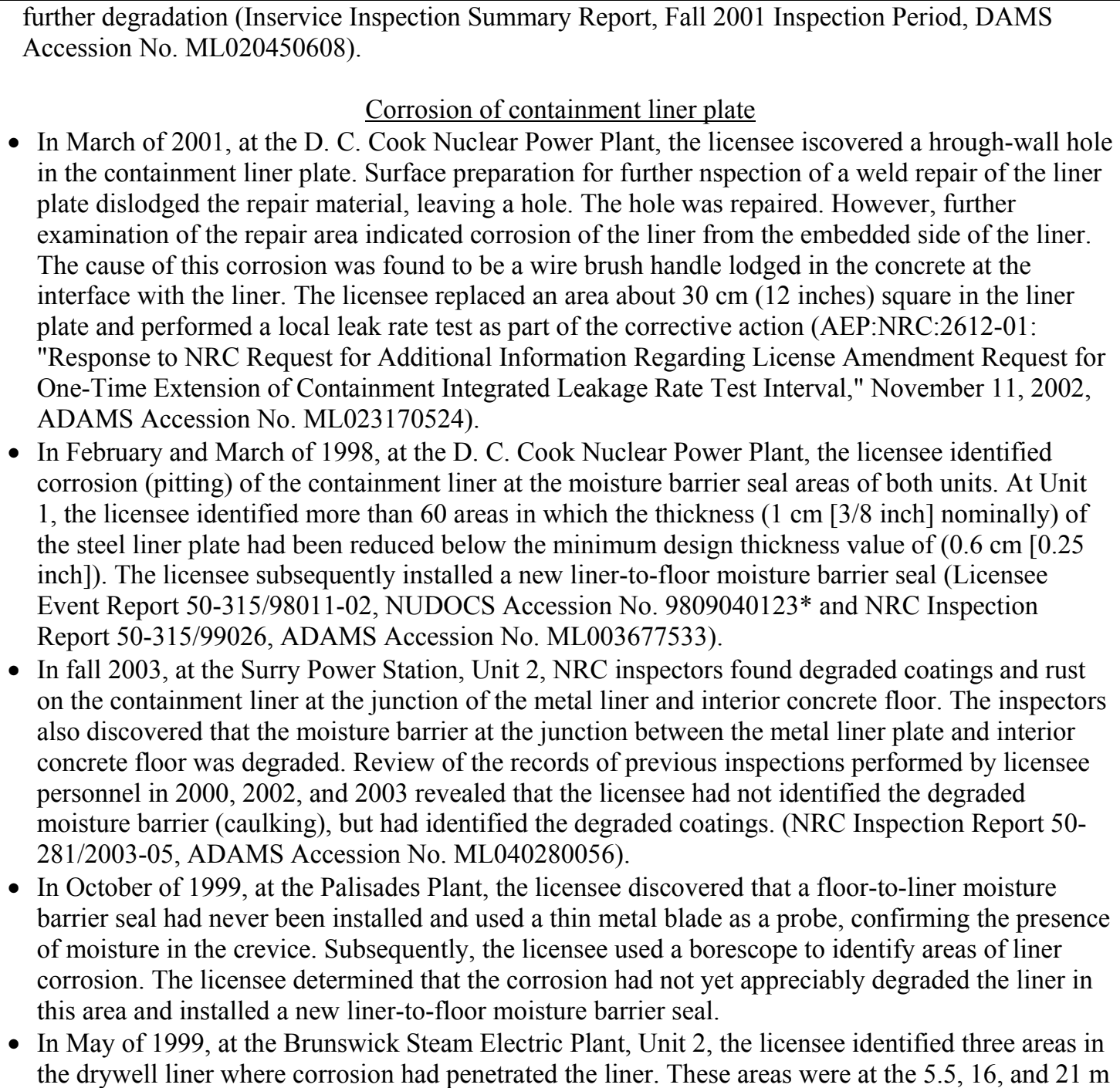 & \\
\hline
\end{tabular}




\begin{tabular}{|c|c|c|c|c|}
\hline Type & No. & Title & Topic & $\begin{array}{c}\text { SPC } \\
\text { Affected } \\
\end{array}$ \\
\hline & & & $\begin{array}{l}\text { (18, 52, and } 70 \text { feet) elevations. At the } 16 \mathrm{~m} \text { elevation, the wall had corroded from the outside to the } \\
\text { inside surface. At the } 21 \mathrm{~m} \text { elevation, the wall had corroded from the inside to the outside surface. } \\
\text { At the } 5.5 \mathrm{~m} \text { elevation, the direction of the through-wall corrosion could not be determined. The } \\
\text { liner corrosion was a result of foreign materials embedded in the concrete containment adjacent to } \\
\text { the liner. One hole in the liner was adjacent to a leather work glove found buried in the concrete, } \\
\text { while the other two hole locations were adjacent to wood found buried in the concrete (NRC } \\
\text { Inspection Report 50-324/99-03, NUDOCS No. 9906170114*). } \\
\text { - In December, 1996, at the H.B. Robinson Steam Electric Plant, Unit 2, an NRC inspector identified } \\
\text { degraded caulking and insulation sheathing panels during a containment walkdown. The vertical } \\
\text { portion of the containment liner at Robinson is protected by Vinylcel insulation, a polyvinyl } \\
\text { chloride material, and a metal sheathing material. The licensee determined that a portion of this } \\
\text { insulation sheathing material was loose and that some of the caulking between the sheathing panels } \\
\text { was deteriorated. After examination during subsequent refueling outages, the licensee determined } \\
\text { that the protective coating for the containment liner was degraded and that while some corrosion of } \\
\text { the containment liner had occurred, the liner met design requirements. The licensee restored the } \\
\text { coating and insulation panels (NRC Inspection Reports 50-261/96-14, NUDOCS Accession No } \\
9702110115^{*} \text { and 50-261/98-02, NUDOCS Accession No. 9805050171*). }\end{array}$ & \\
\hline IN & $2004-08$ & $\begin{array}{l}\text { Reactor } \\
\text { Coolant } \\
\text { Pressure } \\
\text { Boundary } \\
\text { Leakage } \\
\text { Attributabl } \\
\text { e to } \\
\text { Propagatio } \\
\mathrm{n} \text { of } \\
\text { Cracking in } \\
\text { Reactor } \\
\text { Vessel } \\
\text { Nozzle } \\
\text { Welds }\end{array}$ & $\begin{array}{l}\text { The U.S. Nuclear Regulatory Commission (NRC) is issuing this information notice to alert addressees } \\
\text { to cracking identified in the nozzle-to-cap weld of control rod drive (CRD) return line penetration N10 } \\
\text { at Pilgrim Nuclear Power Station (Pilgrim Station). The NRC expects recipients to review the } \\
\text { information in this notice for applicability to their facilities and consider actions, as appropriate, to } \\
\text { avoid similar problems. However, suggestions contained in this } \\
\text { information notice do not constitute NRC requirements and, therefore, do not require any specific } \\
\text { action or written response. } \\
\text { Discussion } \\
\text { During a planned outage on September } 30,2003 \text {, the licensee for Pilgrim Station began performing } \\
\text { drywell inspections to identify and make necessary repairs to reduce drywell leakage. On October } 1 \text {, } \\
\text { 2003, the licensee's drywell inspections revealed leakage from the nozzle-to-cap weld area of } \\
\text { penetration N10. The licensee concluded that the leakage was a contributor to the unidentified drywell } \\
\text { leakage. }\end{array}$ & $\begin{array}{l}\text { RPV nozzle } \\
\text { penetrations }\end{array}$ \\
\hline IN & $2004-05$ & $\begin{array}{l}\text { Spent Fuel } \\
\text { Pool } \\
\text { Leakage to } \\
\text { Onsite }\end{array}$ & $\begin{array}{l}\text { The U.S. Nuclear Regulatory Commission (NRC) is issuing this information notice to inform } \\
\text { addressees of the recent identification of a longstanding leak to onsite groundwater from the spent fuel } \\
\text { pool of an operating pressurized water reactor facility. It is expected that recipients will review the } \\
\text { information for applicability to their facilities and consider actions, as appropriate, to avoid similar }\end{array}$ & $\begin{array}{l}\text { Spent fuel } \\
\text { pool } \\
\text { structure }\end{array}$ \\
\hline
\end{tabular}




\begin{tabular}{|c|c|c|c|c|}
\hline Type & No. & Title & Topic & $\begin{array}{c}\text { SPC } \\
\text { Affected } \\
\end{array}$ \\
\hline & & $\begin{array}{l}\text { Groundwat } \\
\text { er }\end{array}$ & 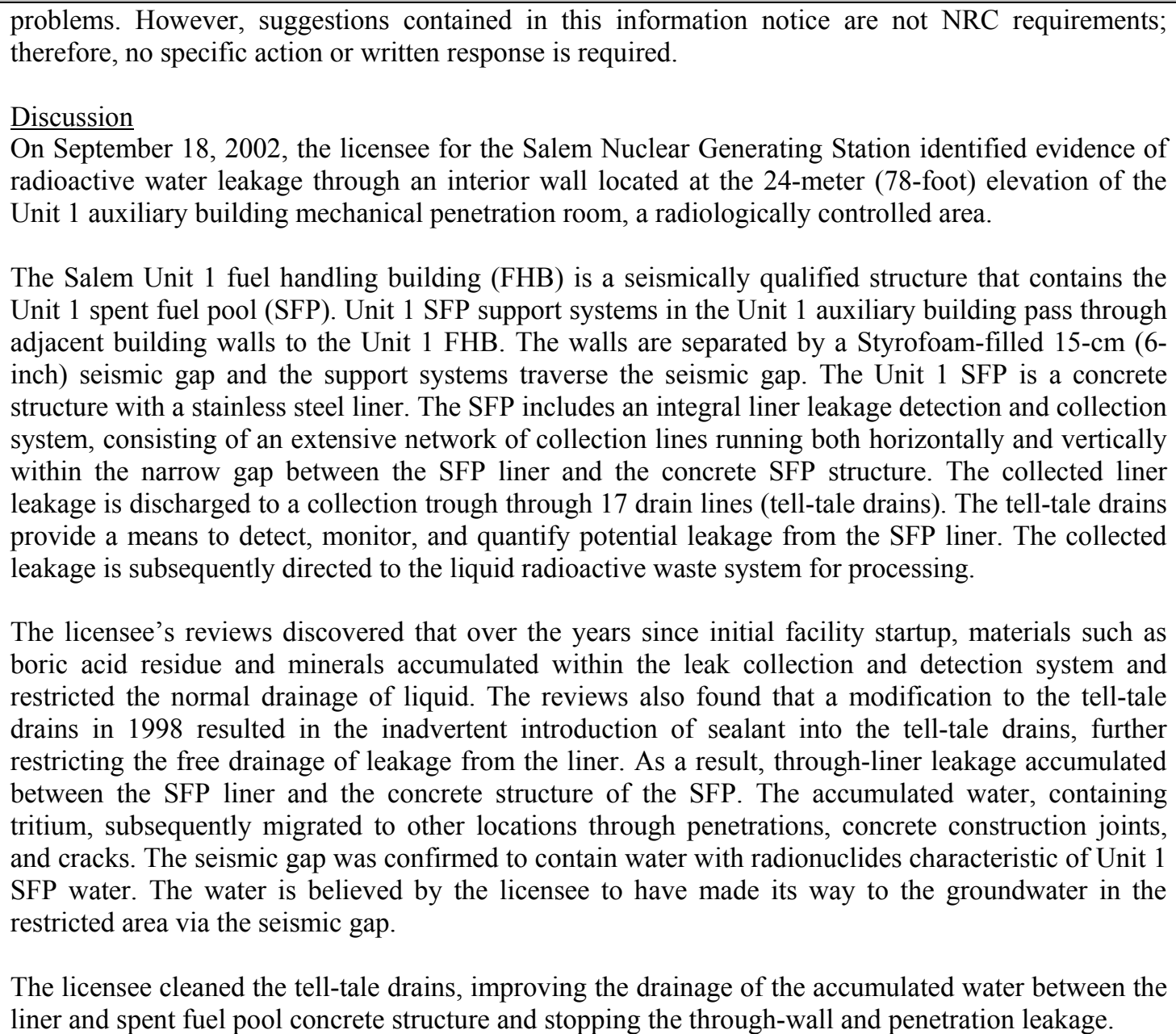 & \\
\hline IN & 2004-01 & $\begin{array}{l}\text { Auxiliary } \\
\text { Feedwater } \\
\text { Pump }\end{array}$ & $\begin{array}{l}\text { The U.S. Nuclear Regulatory Commission (NRC) is issuing this information notice to inform } \\
\text { addressees of the potential common cause failure of auxiliary feedwater pumps because of fouling of } \\
\text { pump recirculation line flow orifices. It is expected that recipients will review the information for }\end{array}$ & $\begin{array}{l}\text { Filter } \\
\text { plugging/foul } \\
\text { ing }\end{array}$ \\
\hline
\end{tabular}




\begin{tabular}{|c|c|c|c|c|}
\hline Type & No. & Title & Topic & $\begin{array}{c}\text { SPC } \\
\text { Affected }\end{array}$ \\
\hline & & $\begin{array}{l}\text { Recirculati } \\
\text { on Line } \\
\text { Orifice } \\
\text { Fouling - } \\
\text { Potential } \\
\text { Common } \\
\text { Cause } \\
\text { Failure }\end{array}$ & $\begin{array}{l}\text { applicability to their facilities and consider actions, as appropriate, to avoid similar problems. } \\
\text { However, suggestions in this information notice are not NRC requirements; therefore no specific } \\
\text { action or written response is required. } \\
\text { Discussion } \\
\text { Point Beach Nuclear Plant (PBNP) is a two unit site. Each unit has a turbine-driven AFW pump } \\
\text { (pumps 1P29 and 2P29) which can supply water to both steam generators. Additionally, the plant has } \\
\text { two motor-driven AFW pumps (pumps P38A and P38B) each of which can be aligned to a steam } \\
\text { generator in each unit. Each pump has a recirculation line back to the condensate storage tanks (CSTs) } \\
\text { to ensure minimum flow to prevent hydraulic instabilities and dissipate pump heat. The recirculation } \\
\text { line contained a pressure reducing, flow restricting orifice. } \\
\text { The RO used a multi-stage, anti-cavitation trim package installed in the body of a globe valve to limit } \\
\text { flow. This style of orifice or flow restrictor was installed in the AFW recirculation lines at PBNP in } \\
\text { the past few years to eliminate cavitation caused by the old orifices. This type of flow restrictor used } \\
\text { very small channels and holes in each stage combined with a tortuous path to limit flow and prevent } \\
\text { cavitation. } \\
\text { After removal of the orifice internals, partial blockage was observed in } 24 \text { of the } 54 \text { holes in the } \\
\text { outermost sleeve. No particles were found on any of the inner sleeves. Samples of the particles } \\
\text { removed from the orifice were retained for analysis. A boroscope inspection of the recirculation piping } \\
\text { at the orifice location revealed no evidence of debris. Following cleaning and reassembly, the orifice } \\
\text { was reinstalled and the P38A AFW pump was successfully retested. }\end{array}$ & \\
\hline IN & $2003-13$ & $\begin{array}{l}\text { Steam } \\
\text { Generator } \\
\text { Tube } \\
\text { Degradatio } \\
\text { n at Diablo } \\
\text { Canyon }\end{array}$ & $\begin{array}{l}\text { The U.S. Nuclear Regulatory Commission is issuing this information notice to inform addressees } \\
\text { about findings from a recent steam generator tube inspection at the Diablo Canyon Power Plant, Unit } 2 \\
\text { (DCPP-2). The NRC anticipates that recipients will review the information for applicability to their } \\
\text { facilities and consider taking actions, as appropriate, to avoid similar problems. However, no specific } \\
\text { action or written response is required. } \\
\text { Discussion } \\
\text { DCPP-2 has four Westinghouse model } 51 \text { steam generators (SGs), with } 7 / 8 \text { inch outside diameter } \\
\text { (OD), mill-annealed Alloy } 600 \text { tubing and drilled hole carbon steel tube support plates. The model } 51 \\
\text { steam generator has } 45 \text { rows of tubes, with row } 1 \text { having the smallest bend radii in the U-bend area. } \\
\text { During Operating Cycle } 11 \text {, a small steam generator tube leak (less than or equal to approximately } 6.5 \\
\text { gallons per day) was present at DCPP-2. During the } 2003 \text { refueling outage, Pacific Gas \& Electric }\end{array}$ & $\begin{array}{l}\text { Steam } \\
\text { generators }\end{array}$ \\
\hline
\end{tabular}




\begin{tabular}{|c|c|c|c|c|}
\hline Туре & No. & Title & Topic & $\begin{array}{c}\text { SPC } \\
\text { Affected } \\
\end{array}$ \\
\hline & & & 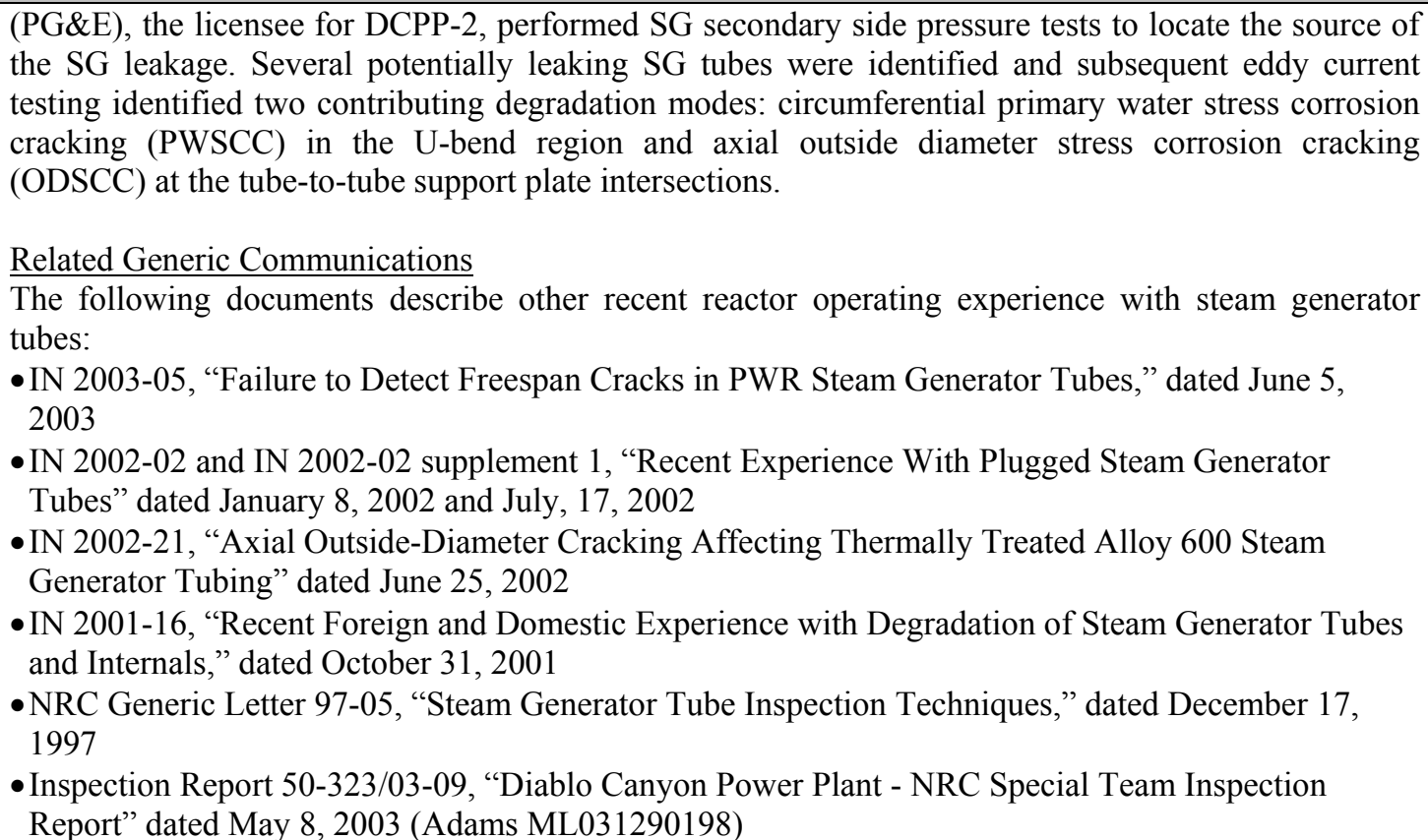 & \\
\hline IN & $\begin{array}{l}2003- \\
11 \mathrm{~s} 1 \\
\text { and } \\
\text { original } \\
2003-11\end{array}$ & $\begin{array}{l}\text { Leakage } \\
\text { Found on } \\
\text { Bottom- } \\
\text { Mounted } \\
\text { Instrument } \\
\text { ation } \\
\text { Nozzles }\end{array}$ & $\begin{array}{l}\text { The U.S. Nuclear Regulatory Commission (NRC) is issuing this information notice (IN) to alert } \\
\text { addressees to indications of leakage in the form of boron deposits discovered on bottom mounted } \\
\text { instrumentation (BMI) nozzles at South Texas Project Unit } 1 \text { (STP Unit 1). This supplement } \\
\text { specifically provides additional information regarding the STP Unit } 1 \text { licensee's root cause analyses, } \\
\text { as discussed in licensee's final licensee event report on this topic, dated October 15, } 2003 \text { (ADAMS } \\
\text { Accession No. ML032950483). It is expected that the recipients of this IN will review the information } \\
\text { for applicability to their facilities and consider actions, as appropriate, to avoid similar problems. } \\
\text { However, suggestions contained in this IN are not NRC requirements; therefore, no specific action or } \\
\text { written response is required. } \\
\text { Discussion } \\
\text { An extensive description of the STP Unit } 1 \text { BMI penetration leakage event was given in IN 2003-11, } \\
\text { "Leakage Found on Bottom-Mounted Instrumentation Nozzles," dated August 13, 2003. The relevant } \\
\text { pre-August } 2003 \text { information is summarized herein. In April 2003, the STP Unit } 1 \text { licensee identified }\end{array}$ & $\begin{array}{l}\text { RPV } \\
\text { instrumentati } \\
\text { on nozzles }\end{array}$ \\
\hline
\end{tabular}




\begin{tabular}{|c|c|c|c|c|}
\hline Type & No. & Title & Topic & $\begin{array}{c}\text { SPC } \\
\text { Affected } \\
\end{array}$ \\
\hline & & & $\begin{array}{l}\text { small boron deposits around two of the } 58 \text { STP Unit } 1 \text { BMI penetrations (penetrations } 1 \text { and } 46 \text { ), the } \\
\text { only evidence of BMI nozzle penetration leakage reported by a U.S. facility to date. The STP Unit } 1 \\
\text { BMI penetrations were constructed from an drilled Inconel } 600 \text { bar stock connected to the reactor } \\
\text { vessel lower head by an Inconel } 82 / 182 \text { J-groove weld. The licensee's subsequent nondestructive } \\
\text { examination (NDE) campaign, which included ultrasonic test (UT), visual, and eddy current testing, } \\
\text { resulted in the identification of three axially oriented cracklike indications in the penetration } 1 \text { nozzle } \\
\text { wall and two axially oriented cracklike indications in the penetration } 46 \text { nozzle wall. }\end{array}$ & \\
\hline IN & $2003-08$ & $\begin{array}{l}\text { Potential } \\
\text { Flooding } \\
\text { Through } \\
\text { Unsealed } \\
\text { Concrete } \\
\text { Floor } \\
\text { Cracks }\end{array}$ & $\begin{array}{l}\text { The Nuclear Regulatory Commission (NRC) is issuing this information notice to inform addressees of } \\
\text { observed flooding in a room containing safety-related panels and equipment as a result of fire water } \\
\text { seepage through unsealed concrete floor cracks. It is expected that recipients will review the } \\
\text { information for applicability to their facilities and consider actions as appropriate to avoid similar } \\
\text { problems. However, suggestions contained in this NRC information notice are not NRC requirements; } \\
\text { therefore, no specific action or written response is required. } \\
\text { Discussion } \\
\text { On May } 3,2002 \text {, at Energy Northwest's Columbia Generating Station, } 15 \text { to } 20 \text { gallons of water } \\
\text { spilled from a firewater drain line onto the floor of the radwaste building } 484 \text { ' elevation cable } \\
\text { spreading room. A small amount of this water leaked down into the remote shutdown room and the } \\
\text { Division II switchgear room, which is located below the cable spreading room floor. The licensee } \\
\text { determined that the pathway for the leakage was through cracks in the concrete floor. }\end{array}$ & $\begin{array}{l}\text { Concrete } \\
\text { floor - cracks }\end{array}$ \\
\hline IN & $2003-05$ & $\begin{array}{l}\text { Failure to } \\
\text { Detect } \\
\text { Freespan } \\
\text { Cracks in } \\
\text { PWR } \\
\text { Steam } \\
\text { Generator } \\
\text { Tubes }\end{array}$ & $\begin{array}{l}\text { This information notice (IN) is being provided to inform licensees of a recent problem experienced at } \\
\text { Comanche Peak Unit } 1 \text { concerning the detection of freespan outside diameter stress corrosion cracking } \\
\text { (ODSCC) in steam generator (SG) tubes. This has led to tube integrity performance criteria not being } \\
\text { met as defined in Nuclear Energy Institute (NEI) 97-06, "Steam Generator Program Guidelines." The } \\
\text { NRC anticipates that recipients will review the information for applicability to their facilities and } \\
\text { consider taking appropriate actions. However, suggestions contained in this IN do not constitute NRC } \\
\text { requirements; therefore, no specific action or written response is required. } \\
\text { Discussion } \\
\text { Comanche Peak Unit } 1 \text { is a four-loop Westinghouse PWR with four Westinghouse Model D4 } \\
\text { recirculating SGs (1, } 2,3,4 \text { ). Each SG contains } 4578 \text { mill- annealed Alloy } 600 \text { tubes, which are } \\
\text { nominally } 0.750 \text { inch in diameter and have a nominal wall thickness of } 0.043 \text { inch. The tubes are } \\
\text { supported by a number of carbon steel tube support plates with circular holes and by V-shaped } \\
\text { chrome-plated Alloy } 600 \text { anti-vibration bars (AVBs). Comanche Peak Unit } 1 \text { was shut down } \\
\text { approximately } 1 \text { week prior to its scheduled refueling outage as a result of a primary-to-secondary }\end{array}$ & $\begin{array}{l}\text { Steam } \\
\text { Generators }\end{array}$ \\
\hline
\end{tabular}




\begin{tabular}{|c|c|c|c|c|}
\hline Туре & No. & Title & Topic & $\begin{array}{c}\text { SPC } \\
\text { Affected }\end{array}$ \\
\hline & & & $\begin{array}{l}\text { leak. A 5- to 15-gallon-per-day (gpd) leak was first observed in SG } 2 \text { on September 26, 2002. Over the } \\
\text { next } 2 \text { days, the leakage spiked to higher values several times. On September 28, 2002, after a leakage } \\
\text { spike to } 52 \text { gpd, the licensee elected to shut down the plant and to commence refueling (1RF09). After } \\
\text { shutting down the plant, the licensee began inspecting the SG tubes with eddy current testing } \\
\text { techniques. }\end{array}$ & \\
\hline IIN & $2003-02$ & $\begin{array}{l}\text { Recent } \\
\text { Experience } \\
\text { With } \\
\text { Reactor } \\
\text { Coolant } \\
\text { System } \\
\text { Leakage } \\
\text { And Boric } \\
\text { Acid } \\
\text { Corrosion }\end{array}$ & $\begin{array}{l}\text { The U.S. Nuclear Regulatory Commission (NRC) is issuing this information notice to inform } \\
\text { addressees of recently observed reactor coolant leakage at two pressurized water reactor facilities, one } \\
\text { of which resulted in the subsequent degradation of the reactor pressure vessel head. It is expected that } \\
\text { recipients will review the information for applicability to their facilities and consider actions, as } \\
\text { appropriate, to avoid similar problems. However, suggestions in this information notice are not NRC } \\
\text { requirements; therefore no specific action or written response is required. } \\
\text { Discussion } \\
\text { A number of mechanical and welded connections exist above the reactor pressure vessel head that, } \\
\text { historically, have leaked at a number of plants. This leakage of borated water may lead to degradation } \\
\text { of the low alloy steel reactor vessel head by boric acid corrosion. At Sequoyah Unit } 2 \text { (December } 26 \text {, } \\
\text { 2002), the leakage resulted in relatively minor degradation of the reactor vessel head. At Comanche } \\
\text { Peak Unit } 1 \text { (November } 30,2002 \text { ), the leakage resulted in no apparent degradation of the RCS pressure } \\
\text { boundary. In the Sequoyah Unit } 2 \text { and Comanche Peak Unit } 1 \text { events, the unidentified reactor coolant } \\
\text { leakage had not shown a discernible increase from the very low levels that typically occur at a PWR } \\
\text { facility. }\end{array}$ & $\begin{array}{l}\text { RPV - head } \\
\text { connection/p } \\
\text { enetration }\end{array}$ \\
\hline IN & $\begin{array}{l}2002-26 \\
\text { s1, s2, } \\
\& \\
\text { original } \\
2002-26\end{array}$ & $\begin{array}{l}\text { Additional } \\
\text { Flow- } \\
\text { Induced } \\
\text { Vibration } \\
\text { Failures } \\
\text { after a } \\
\text { Recent } \\
\text { Power } \\
\text { Uprate }\end{array}$ & $\begin{array}{l}\text { The U.S. Nuclear Regulatory Commission (NRC) is issuing this supplement to a previously issued } \\
\text { information notice (IN) to alert addressees to the failure of the steam dryer and other plant components } \\
\text { at Quad Cities Nuclear Power Station, Unit } 1 \text { (QC-1), a boiling water reactor (BWR), during } \\
\text { operations following a power uprate. The NRC expects that the recipients will review the information } \\
\text { for applicability to their facilities and consider actions, as appropriate, to avoid similar problems. } \\
\text { However, suggestions contained in this information notice are not NRC requirements. Therefore, no } \\
\text { specific action or written response is required. } \\
\text { Discussion } \\
\text { As discussed in IN 2002-26, "Failure of Steam Dryer Cover Plate After a Recent Power Uprate" } \\
\text { (ML022530291), a cover plate on the outside of the steam dryer at Quad Cities Nuclear Power Station, } \\
\text { Unit } 2 \text { (QC-2), broke loose in June } 2002 \text { and caused pieces of the dryer to be swept down the main } \\
\text { steamline. Before the unit was shut down in 2002, steam dryer degradation was indicated by an } \\
\text { increase in moisture carryover and minor perturbations in reactor pressure, water level, and steam }\end{array}$ & $\begin{array}{l}\text { RPV - steam } \\
\text { dryer cover } \\
\text { plate, hood, } \\
\text { \& other areas } \\
\text { - fatigue }\end{array}$ \\
\hline
\end{tabular}




\begin{tabular}{|c|c|c|c|c|}
\hline Type & No. & Title & Topic & $\begin{array}{c}\text { SPC } \\
\text { Affected }\end{array}$ \\
\hline & & & $\begin{array}{l}\text { flow. The licensee evaluated the cause of the steam dryer cover plate failure and determined that the } \\
\text { failure of the plate was due to high-cycle fatigue. } \\
\text { The second failure of the steam dryer in May } 2003 \text { at QC-2 was discussed in IN 2002-26, Supplement } \\
\text { 1, "Additional Failure of Steam Dryer After a Recent Power Uprate" (ML031980434). Inspection of } \\
\text { the dryer revealed (1) through-all cracks (about } 90 \text { inches long) in the vertical and horizontal portions } \\
\text { of the outer bank hood, 90-degree side, (2) one vertical and two diagonal internal braces detached from } \\
\text { the outer bank hood, 90-degree side, (3) one severed vertical internal brace on the outer bank hood, } \\
\text { 270-degree side, and (4) three cracked tie bars on top of the dryer. The licensee believes the most } \\
\text { probable cause of the failure of the steam dryer in QC-2 is low-frequency, high-cycle fatigue driven by } \\
\text { flow induced vibrations associated with the higher steam flows present during EPU operating } \\
\text { conditions. } \\
\text { In late October } 2003 \text { at QC-1, the licensee observed changes in main steamline flows, steamline } \\
\text { pressure drop, and increasing moisture carryover measurements. On November 12, the licensee shut } \\
\text { down QC-1 to inspect the steam dryer and identified significant damage to several areas. }\end{array}$ & \\
\hline IN & $\begin{array}{l}2002-02 \\
\text { s1 }\end{array}$ & $\begin{array}{l}\text { Recent } \\
\text { Experience } \\
\text { With } \\
\text { Plugged } \\
\text { Steam } \\
\text { Generator } \\
\text { Tubes }\end{array}$ & $\begin{array}{l}\text { The U.S. Nuclear Regulatory Commission (NRC) is issuing this information notice (IN) to inform } \\
\text { addressees of findings from recent inspections and examinations of steam generator tubes at Oconee } \\
\text { Nuclear Station Unit } 1 \text { (ONS-1). The NRC anticipates that recipients will review the information for } \\
\text { applicability to their facilities and consider taking actions, as appropriate, to avoid similar problems. } \\
\text { However, suggestions contained in this information notice do not constitute NRC requirements; } \\
\text { therefore, no specific action or written response is required. } \\
\text { Discussion } \\
\text { Potential severance of plugged steam generator tubes was discussed in IN 2002-02, "Recent } \\
\text { Experience With Plugged Steam Generator Tubes," (ML013480327) as a result of inspection findings } \\
\text { at Three Mile Island Unit } 1 \text { (TMI-1) during the fall } 2001 \text { refueling outage. At TMI-1, a plugged tube, } \\
\text { located on the periphery of the tube bundle, severed near the secondary side of the upper tubesheet and } \\
\text { damaged four adjacent in-service (i.e., nonplugged) tubes. The preliminary laboratory investigation of } \\
\text { the severed tube found signs of high cycle fatigue, ductile failure, and outside-diameter-initiated } \\
\text { intergranular attack (IGA). In addition, the tube diameter was greater than the nominal tube diameter, } \\
\text { indicating that the severed tube had swollen. The licensee determined that the most likely cause of } \\
\text { failure was fatigue caused by flow-induced vibration of the swollen and restrained tube. } \\
\text { On March } 25,2002 \text {, ONS-1 was shut down for a refueling outage. In addition to the standard steam }\end{array}$ & $\begin{array}{l}\text { Steam } \\
\text { Generators }\end{array}$ \\
\hline
\end{tabular}




\begin{tabular}{|c|c|c|c|c|}
\hline Type & No. & Title & Topic & $\begin{array}{c}\text { SPC } \\
\text { Affected }\end{array}$ \\
\hline & & & $\begin{array}{l}\text { generator tube inspections, the licensee performed supplemental inspections of plugged tubes in both } \\
\text { steam generators. These supplemental inspections were performed to address the TMI-1 plugged tube } \\
\text { severance event (discussed in Information Notice 2002-02). While inspecting two tubes in the B steam } \\
\text { generator, the licensee identified signs of wear on the outside tube surface near the secondary face of } \\
\text { the lower tubesheet. The circumferential location of the wear on these two tubes indicated that the } \\
\text { wear was most likely due to impact from an adjacent tube. Subsequent visual inspection from the } \\
\text { secondary side of the steam generator indicated that tube one of the tubes was completely severed at } \\
\text { the secondary side (i.e., top) of the lower tubesheet. }\end{array}$ & \\
\hline IN & $\begin{array}{l}2002- \\
21, \text { s1 \& } \\
\text { original } \\
02-21\end{array}$ & $\begin{array}{l}\text { Axial } \\
\text { Outside- } \\
\text { Diameter } \\
\text { Cracking } \\
\text { Affecting } \\
\text { Thermally } \\
\text { Treated } \\
\text { Alloy } 600 \\
\text { Steam } \\
\text { Generator } \\
\text { Tubing }\end{array}$ & $\begin{array}{l}\text { The U.S. Nuclear Regulatory Commission (NRC) is issuing this supplement to IN 2002-21 to inform } \\
\text { addressees of the root cause assessment for the axially oriented outside-diameter crack indications in } \\
\text { the thermally treated Alloy } 600 \text { steam generator (SG) tubing at Seabrook. It is expected that recipients } \\
\text { will review the information for applicability to their facilities and consider actions, as appropriate, to } \\
\text { avoid similar problems. However, suggestions contained in this information notice are not NRC } \\
\text { requirements; therefore, no specific action or written response is required. } \\
\text { Discussion } \\
\text { During the eighth refueling outage, } 42 \text { eddy current indications in } 15 \text { "low row" tubes (tubes in rows } 1 \\
\text { through 10) were identified and classified as potential axially oriented outside diameter stress } \\
\text { corrosion cracks (ODSCC). All indications were in one steam generator and all indications were } \\
\text { located in the region where the tube passes through a TSP (i.e., tube-to-tubesupport-plate intersection). } \\
\text { Both hot and cold leg tubes were affected. No indications were observed at the top of the tubesheet. } \\
\text { This issue was discussed in NRC IN 2002-21, "Axial Outside-Diameter Cracking Affecting Thermally } \\
\text { Treated Alloy } 600 \text { Steam Generator Tubing", issued June 25, 2002 (ADAMS Accession No. } \\
\text { ML021770094). }\end{array}$ & $\begin{array}{l}\text { Steam } \\
\text { Generators }\end{array}$ \\
\hline IIN & $2002-13$ & $\begin{array}{l}\text { Possible } \\
\text { Indicators } \\
\text { of Ongoing } \\
\text { Reactor } \\
\text { Pressure } \\
\text { Vessel } \\
\text { Head } \\
\text { Degradatio } \\
\text { n }\end{array}$ & $\begin{array}{l}\text { The U.S. Nuclear Regulatory Commission is issuing this information notice on recent Davis-Besse } \\
\text { experience to alert addressees to possible indicators of reactor coolant pressure boundary degradation } \\
\text { including degradation of the reactor pressure vessel (RPV) head material. The NRC anticipates that } \\
\text { recipients will review this information for applicability to their facilities and consider taking } \\
\text { appropriate actions. However, the suggestions contained in this information notice do not constitute } \\
\text { NRC requirements and, therefore, no specific action or written response is required. } \\
\text { Discussion } \\
\text { The Davis-Besse nuclear power plant recently discovered a significant cavity in the RPV head on the } \\
\text { downhill side of control rod drive nozzle number } 3 \text { and some head wastage behind nozzle number } 2 \text {. In } \\
\text { response, the NRC issued Information Notice } 2002-11 \text {, "Recent Experience With Degradation of }\end{array}$ & RPV head \\
\hline
\end{tabular}




\begin{tabular}{|c|c|c|c|c|}
\hline Туре & No. & Title & Topic & $\begin{array}{c}\text { SPC } \\
\text { Affected }\end{array}$ \\
\hline & & & $\begin{array}{l}\text { Reactor Pressure Vessel Head," on March 12, 2002, and Bulletin 2002-01, "Reactor Pressure Vessel } \\
\text { Head Degradation and Reactor Coolant Pressure Boundary Integrity," on March 18, 2002. NRC also } \\
\text { sent an Augmented Inspection Team (AIT) to the plant to investigate the circumstances of the } \\
\text { degradation of the RPV head material. Through the AIT, several possible indicators of reactor coolant } \\
\text { pressure boundary degradation such as was observed at Davis-Besse were identified. These indicators } \\
\text { include unidentified reactor coolant system (RCS) leakage and containment air cooler (CAC) and } \\
\text { radiation element (RE) filter fouling. } \\
\text { RCS leakage, boron deposits, and corrosion products like ferric oxide in CACs and RE filters may } \\
\text { indicate degradation of the reactor coolant pressure boundary materials. These indicators do not } \\
\text { provide clear evidence of the degradation; however, they may provide an opportunity for licensees to } \\
\text { suspect that degradation is ongoing. The NRC understands that the indications at Davis-Besse were } \\
\text { sometimes complicated by other events (e.g., flange leaks). Nonetheless, in combination with other } \\
\text { indicators, they may provide insights into whether degradation of the reactor coolant pressure } \\
\text { boundary materials is occurring. }\end{array}$ & \\
\hline IN & $2002-11$ & $\begin{array}{l}\text { Recent } \\
\text { Experience } \\
\text { with } \\
\text { Degradatio } \\
n \quad \text { of } \\
\text { Reactor } \\
\text { Pressure } \\
\text { Vessel } \\
\text { Head }\end{array}$ & $\begin{array}{l}\text { The U.S. Nuclear Regulatory Commission is issuing this information notice to inform addressees } \\
\text { about findings from recent inspections and examinations of the reactor pressure vessel (RPV) head at } \\
\text { Davis-Besse Nuclear Power Station. It is expected that recipients will review the information for } \\
\text { applicability to their facilities and consider actions, as appropriate, to avoid similar problems. } \\
\text { However, suggestions contained in this information notice are not NRC requirements; therefore, no } \\
\text { specific action or written response is required. } \\
\text { Discussion } \\
\text { On February 16, 2002, the Davis-Besse facility began a refueling outage that included inspection of } \\
\text { the vessel head penetration (VHP) nozzles, which focused on the inspection of control rod drive } \\
\text { mechanism (CRDM) nozzles, in accordance with the licensee's commitments to NRC Bulletin 2001- } \\
01 \text {, "Circumferential Cracking of Reactor Pressure Vessel Head Penetration Nozzles," which was } \\
\text { issued on August } 3 \text {, 2001. These inspections identified axial indications in three CRDM nozzles, } \\
\text { which had resulted in pressure boundary leakage. Specifically, these indications were identified in } \\
\text { CRDM nozzles } 1,2 \text {, and } 3 \text {, which are located near the center of the RPV head. These findings were } \\
\text { reported to the NRC on February } 27,2002 \text {, and supplemented on March } 5 \text { and March } 9 \text {, 2002. The } \\
\text { licensee decided to repair these three nozzles, as well as two other nozzles that had indications but had } \\
\text { not resulted in pressure boundary leakage. } \\
\text { Related Generic Communications }\end{array}$ & RPV head \\
\hline
\end{tabular}




\begin{tabular}{|c|c|c|c|c|}
\hline Type & No. & Title & Topic & $\begin{array}{c}\text { SPC } \\
\text { Affected }\end{array}$ \\
\hline & & & $\begin{array}{l}\text { - Bulletin 2001-01, "Circumferential Cracking of Reactor Pressure Vessel Head Penetration Nozzles," } \\
\text { August 3, 2001. } \\
\text { - Bulletin 82-02, "Degradation of Threaded Fasteners in the Reactor Coolant Pressure Boundary of } \\
\text { PWR Plants," June 2, 1982. } \\
\text { - Generic Letter 88-05, "Boric Acid Corrosion of Carbon Steel Reactor Pressure Boundary } \\
\text { Components in PWR Plants," March 17, } 1988 . \\
\text { - Generic Letter 97-01, "Degradation of Control Rod Drive Mechanism Nozzles and Other Vessel } \\
\text { Closure Head Penetrations," April 1, 1997. } \\
\text { - Information Notice 80-27, "Degradation of Reactor Coolant Pump Studs," June 11, 1980. } \\
\text { - Information Notice 82-06, "Failure of Steam Generator Primary Side Manway Closure Studs," March } \\
\text { 12, 1982. } \\
\text { - Information Notice 86-108, "Degradation of Reactor Coolant System Pressure Boundary Resulting } \\
\text { from Boric Acid Corrosion," December 29, 1986. } \\
\text { - Information Notice 86-108, Supplement 1, "Degradation of Reactor Coolant System Pressure } \\
\text { Boundary Resulting from Boric Acid Corrosion," April 20, 1987. } \\
\text { - Information Notice 86-108, Supplement 2, "Degradation of Reactor Coolant System Pressure } \\
\text { Boundary Resulting from Boric Acid Corrosion," November 19, 1987. } \\
\text { - Information Notice 86-108, Supplement 3, "Degradation of Reactor Coolant System Pressure } \\
\text { Boundary Resulting from Boric Acid Corrosion," January 5, 1995. } \\
\text { - Information Notice 90-10, "Primary Water Stress Corrosion Cracking of INCONEL 600," February } \\
\text { 23, 1990. } \\
\text { - Information Notice 94-63, "Boric Acid Corrosion of Charging Pump Casing Caused by Cladding } \\
\text { Cracks," August 30, 1994. } \\
\text { - Information Notice 96-11, "Ingress of Demineralizer Resins Increases Potential for Stress Corrosion } \\
\text { Cracking of Control Rod Drive Mechanism Penetrations," February 14, 1996. } \\
\text { Information Notice 2001-05, "Through-Wall Circumferential Cracking of Reactor Pressure Vessel } \\
\text { Head Control Rod Drive Mechanism Penetration Nozzles at Oconee Nuclear Station, Unit } 3, " \text { April } \\
\text { 30, 2001. }\end{array}$ & \\
\hline IN & $2002-02$ & $\begin{array}{l}\text { Recent } \\
\text { Experience } \\
\text { with } \\
\text { Plugged } \\
\text { Steam } \\
\text { Generator }\end{array}$ & $\begin{array}{l}\text { The U.S. Nuclear Regulatory Commission is issuing this information notice to inform addressees } \\
\text { about findings from recent inspections and examinations of steam generator tubes at Three Mile Island } \\
\text { Unit } 1 \text { (TMI-1). The NRC anticipates that recipients will review the information for applicability to } \\
\text { their facilities and consider taking actions, as appropriate, to avoid similar problems. However, } \\
\text { suggestions contained in this information notice do not constitute NRC requirements; therefore, no } \\
\text { specific action or written response is required. }\end{array}$ & $\begin{array}{l}\text { Steam } \\
\text { Generators }\end{array}$ \\
\hline
\end{tabular}




\begin{tabular}{|c|c|c|c|c|}
\hline Туре & No. & Title & Topic & $\begin{array}{c}\text { SPC } \\
\text { Affected }\end{array}$ \\
\hline & & Tubes & $\begin{array}{l}\text { Discussion } \\
\text { On October } 8,2001 \text {, TMI-1 was shut down for a refueling outage. While inspecting the tubes in the B } \\
\text { Steam Generator, the licensee (AmerGen Energy Company, LLC) identified signs of wear near the } \\
\text { upper tubesheet on the outer surface of four tubes on the periphery of the tube bundle. These wear } \\
\text { indications did not appear to have been present during the prior steam generator tube inspections, } \\
\text { which were performed approximately } 2 \text { years earlier. Given the pattern and location of the wear signs, } \\
\text { the licensee suspected that a neighboring plugged tube had caused the wear. The licensee removed the } \\
\text { upper plug in the suspected tube and performed a video inspection. The video inspection revealed that } \\
\text { the tube was severed near the secondary face of the upper tubesheet and in physical contact with the } \\
\text { drilled hole where the tube passed through the } 15^{\text {th }} \text { support plate. Neighboring tubes were not in } \\
\text { contact with the drilled holes at the point where they passed through the } 15^{\text {th }} \text { support plate. }\end{array}$ & \\
\hline IN & $2001-16$ & $\begin{array}{l}\text { Recent } \\
\text { Foreign } \\
\text { and } \\
\text { Domestic } \\
\text { Experience } \\
\text { with } \\
\text { Degradatio } \\
\mathrm{n} \text { of Steam } \\
\text { Generator } \\
\text { Tubes and } \\
\text { Internals }\end{array}$ & $\begin{array}{l}\text { The U.S. Nuclear Regulatory Commission is issuing this information notice to inform addressees } \\
\text { about findings from recent inspections of steam generator tubes and secondary-side internal } \\
\text { components and structures. It is expected that recipients will review the information for applicability } \\
\text { to their facilities and consider actions, as appropriate, to avoid similar problems. However, suggestions } \\
\text { contained in this information notice are not NRC requirements; therefore, no specific action or written } \\
\text { response is required. } \\
\text { Discussion } \\
\text { Foreign Sludge Lancing Experience } \\
\text { In } 1998 \text {, a foreign reactor was shut down for a refueling outage. At the time of the shutdown, there was } \\
\text { no evidence of primary-to-secondary leakage. During the outage, sludge lancing was performed } \\
\text { followed by a bobbin coil probe inspection of } 100 \% \text { of the tubes in all four steam generators. The tube } \\
\text { inspections revealed only minor wall thinning. However, during plant startup following the outage, a } \\
\text { very small primary-to-secondary leak was observed, and the reactor was shut down to investigate its } \\
\text { source. Subsequent inspections identified several degraded steam generator tubes in the second and } \\
\text { third rows of the steam generator tube lane. The degradation consisted of localized loss of the outer } \\
\text { surface of the tubes just above the top of the tubesheet. Extensive wall loss in one of these tubes } \\
\text { resulted in a pinhole-sized perforation of the tube wall. } \\
\text { Degradation of the Calvert Cliffs Unit } 2 \text { Tube Support }\end{array}$ & $\begin{array}{l}\text { Steam } \\
\text { Generators }\end{array}$ \\
\hline
\end{tabular}




\begin{tabular}{|c|c|c|c|c|}
\hline Type & No. & Title & Topic & $\begin{array}{c}\text { SPC } \\
\text { Affected }\end{array}$ \\
\hline & & & 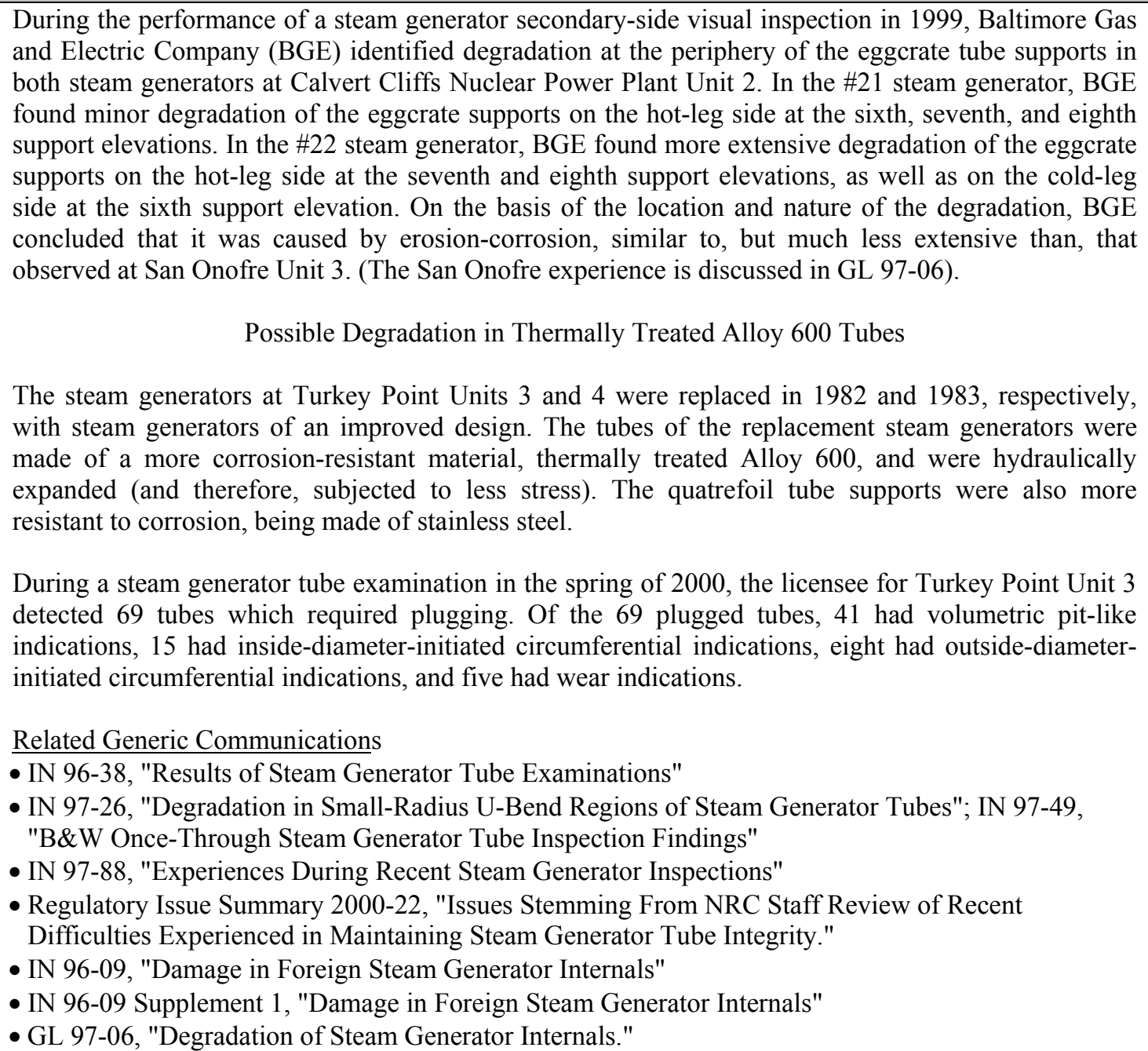 & \\
\hline IN & $2001-09$ & Main & The U.S. Nuclear Regulatory Commission is issuing this information notice (IN) to alert addressees to & Piping \\
\hline
\end{tabular}




\begin{tabular}{|c|c|c|c|c|}
\hline Type & No. & Title & Topic & $\begin{array}{c}\text { SPC } \\
\text { Affected }\end{array}$ \\
\hline & & $\begin{array}{l}\text { Feedwater } \\
\text { System } \\
\text { Degradatio } \\
\mathrm{n} \text { in Safety- } \\
\text { Related } \\
\text { ASME } \\
\text { Code Class } \\
2 \text { Piping } \\
\text { Inside the } \\
\text { Containme } \\
\text { nt of a } \\
\text { Pressurized } \\
\text { Water } \\
\text { Reactor }\end{array}$ & 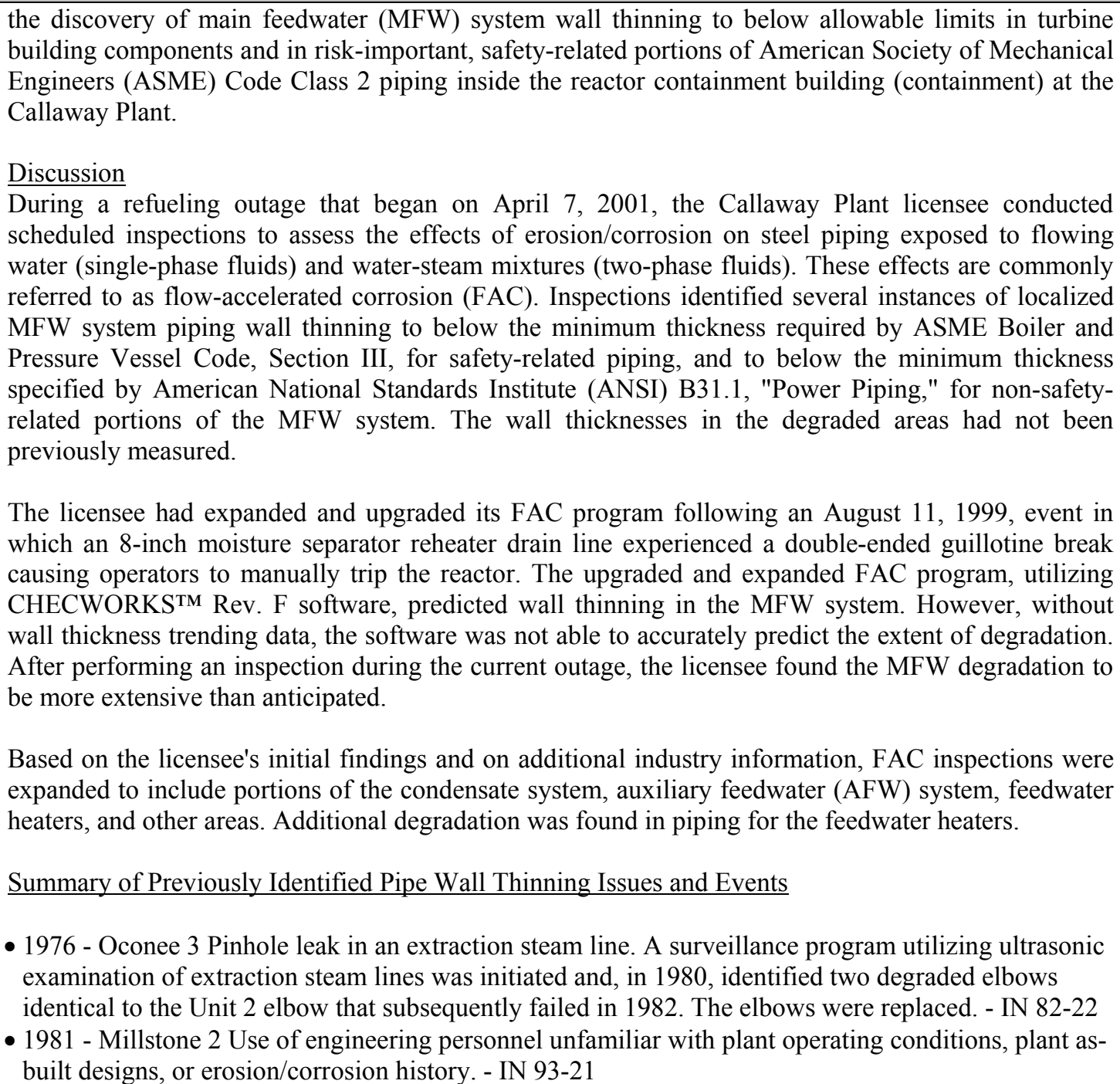 & \\
\hline
\end{tabular}




\begin{tabular}{|c|c|c|c|c|}
\hline Type & No. & Title & Topic & $\begin{array}{c}\text { SPC } \\
\text { Affected }\end{array}$ \\
\hline & & & 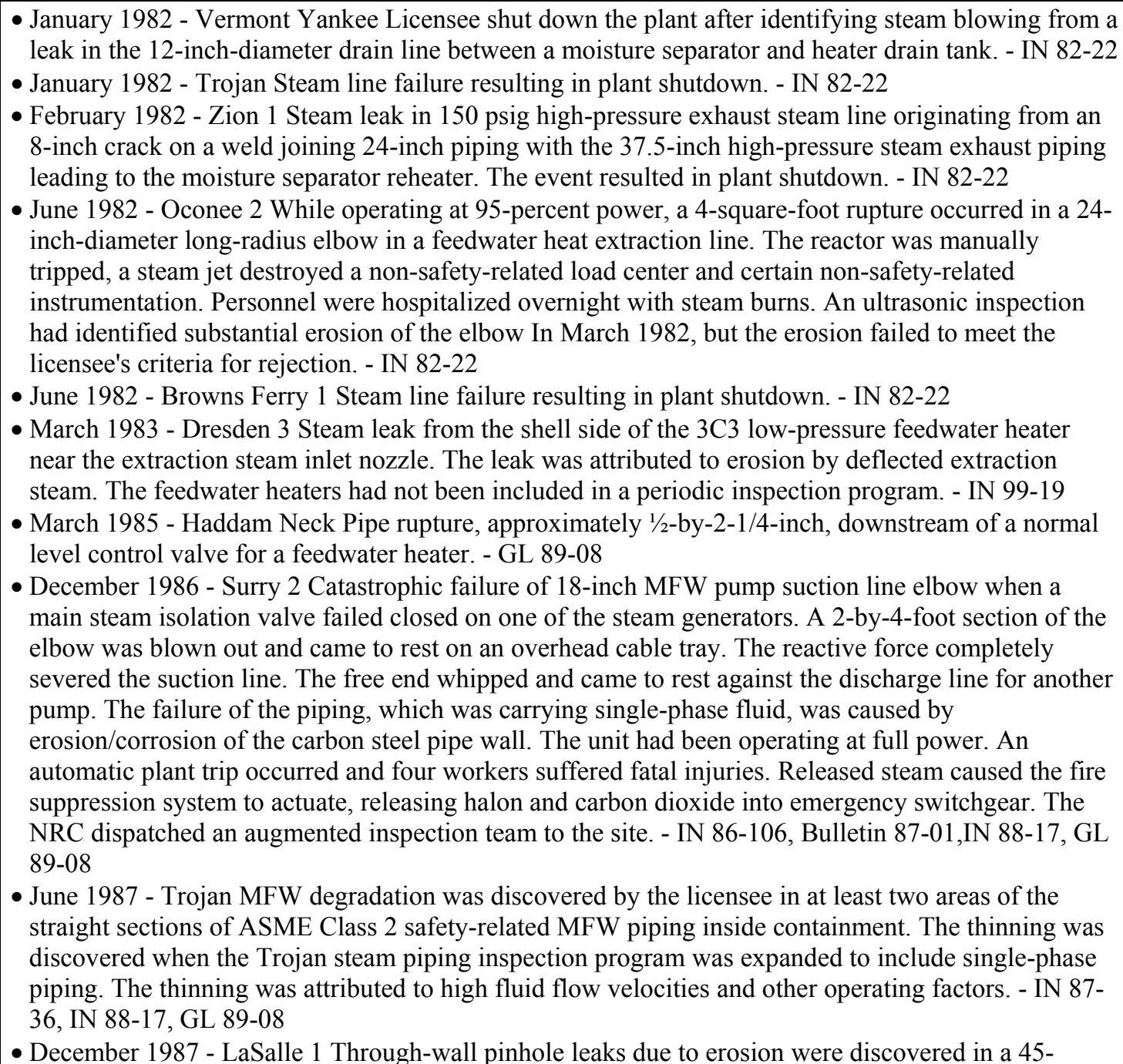 & \\
\hline
\end{tabular}




\begin{tabular}{|c|c|c|c|c|}
\hline Type & No. & Title & Topic & $\begin{array}{c}\text { SPC } \\
\text { Affected }\end{array}$ \\
\hline & & & 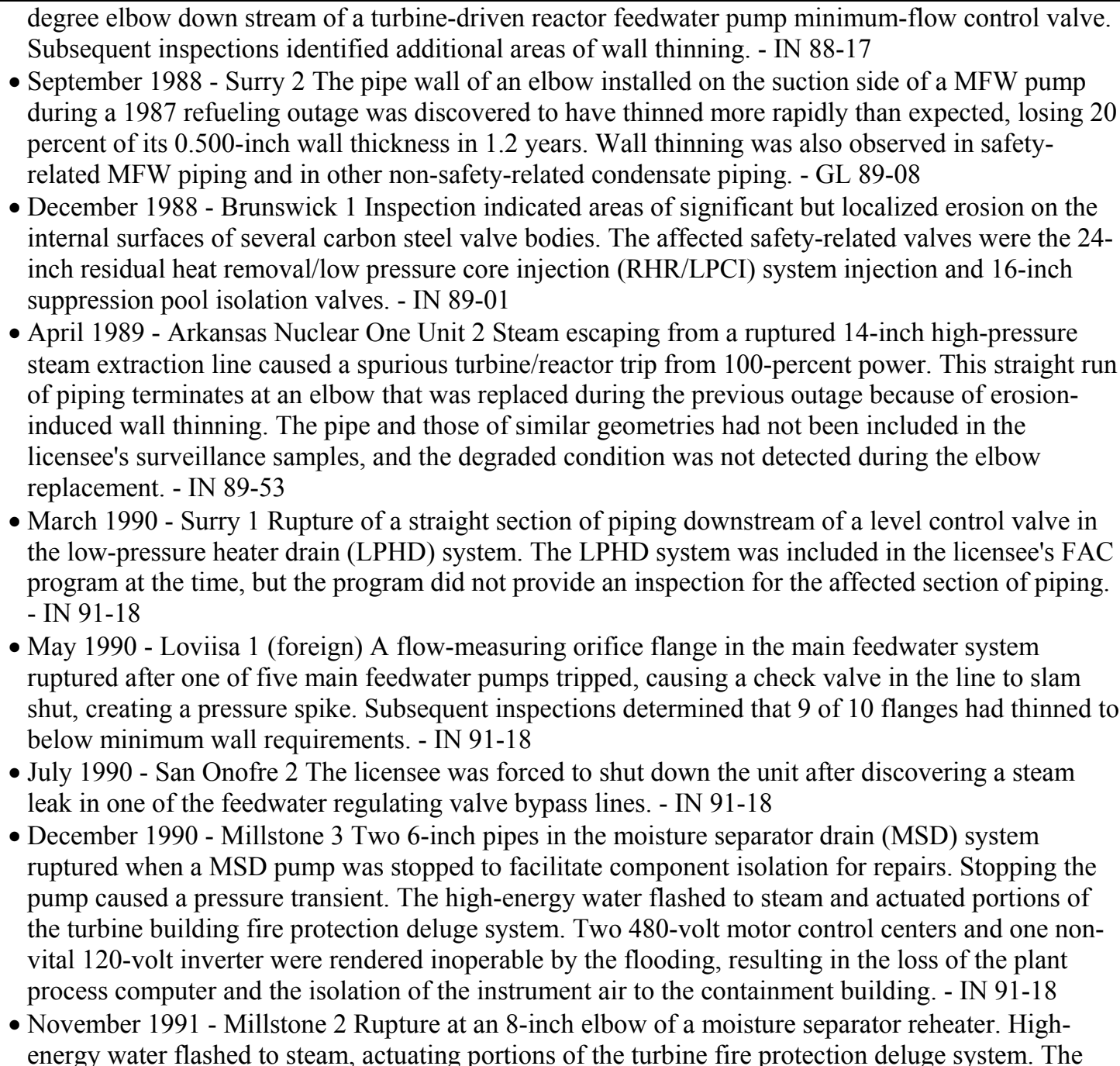 & \\
\hline
\end{tabular}




\begin{tabular}{|c|c|c|c|c|}
\hline Type & No. & Title & Topic & $\begin{array}{c}\text { SPC } \\
\text { Affected } \\
\end{array}$ \\
\hline & & & 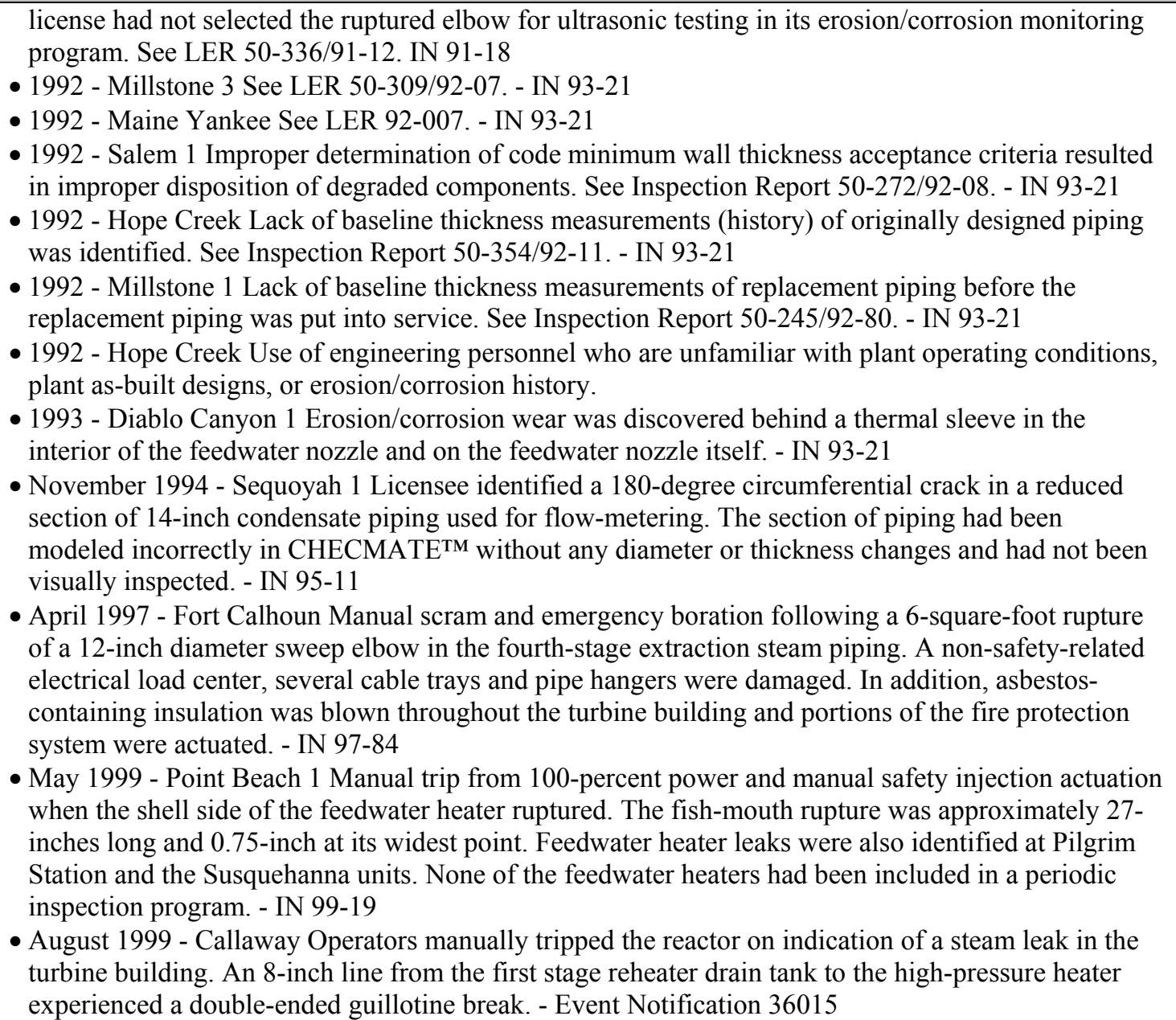 & \\
\hline IN & $2005-01$ & $\begin{array}{l}\text { Through- } \\
\text { Wall } \\
\text { Circumfere }\end{array}$ & $\begin{array}{l}\text { The U.S. Nuclear Regulatory Commission (NRC) is issuing this information notice to alert addressees } \\
\text { to the recent detection of through-wall circumferential cracks in two of the control rod drive } \\
\text { mechanism (CRDM) penetration nozzles and weldments at the Oconee Nuclear Station, Unit } 3\end{array}$ & $\begin{array}{l}\text { RPB head } \\
\text { CRD } \\
\text { penetration }\end{array}$ \\
\hline
\end{tabular}




\begin{tabular}{|c|c|c|c|c|}
\hline Type & No. & Title & Topic & $\begin{array}{c}\text { SPC } \\
\text { Affected }\end{array}$ \\
\hline & & $\begin{array}{l}\text { ntial } \\
\text { Cracking of } \\
\text { Reactor } \\
\text { Pressure } \\
\text { Vessel } \\
\text { Head } \\
\text { Control } \\
\text { Rod Drive } \\
\text { Mechanism } \\
\text { Penetration } \\
\text { Nozzles at } \\
\text { Oconee } \\
\text { Nuclear } \\
\text { Station, } \\
\text { Unit } 3\end{array}$ & $\begin{array}{l}\text { (ONS3). It is expected that recipients will review the information for applicability to their facilities and } \\
\text { consider actions, as appropriate. However, suggestions contained in this information notice are not } \\
\text { NRC requirements; IN 2001-05 } \\
\text { Discussion } \\
\text { On February 18, 2001, with ONS3 in Mode 5, Duke Energy Corporation (the licensee) performed a } \\
\text { visual examination (VT-2) of the outer surface of the unit's reactor pressure vessel (RPV) head to } \\
\text { inspect for indications of borated water leakage. This RPV head inspection was performed as part of a } \\
\text { normal surveillance during a planned maintenance outage. The VT-2 revealed the presence of small } \\
\text { amounts of boric acid residue in the vicinity of nine of the } 69 \text { CRDM penetration nozzles (Figures } 1 \\
\text { and 2). Subsequent nondestructive examinations (NDEs) identified } 47 \text { recordable crack indications in } \\
\text { these nine degraded CRDM penetration nozzles. The licensee initially characterized these flaws as } \\
\text { either axial or below-the-weld circumferential indications, and initiated repairs of the degraded areas. } \\
\text { NDEs of nine additional CRDM penetration nozzles from the same heat of material were conducted } \\
\text { for "extent of condition" purposes, but did not detect recordable indications }\end{array}$ & nozzles \\
\hline IN & $\begin{array}{l}200-17 \\
\text { s2, s1 } \\
\text { and } \\
\text { Original } \\
2000-17\end{array}$ & $\begin{array}{l}\text { Crack in } \\
\text { Weld Area } \\
\text { of Reactor } \\
\text { Coolant } \\
\text { System Hot } \\
\text { Leg Piping } \\
\text { at V. C. } \\
\text { Summer }\end{array}$ & $\begin{array}{l}\text { The U.S. Nuclear Regulatory Commission is issuing this information notice (IN) supplement to } \\
\text { provide updated information about the crack found in a weld in the A loop hot leg pipe in the reactor } \\
\text { coolant system (RCS) at the V. C. Summer Nuclear Station. It is expected that recipients will review } \\
\text { the information for applicability to their facilities and consider actions, as appropriate, to avoid similar } \\
\text { problems. However, no specific action or written response is required. } \\
\text { Discussion } \\
\text { On October 7, 2000, during a containment inspection after entering a refueling outage, the licensee } \\
\text { identified a large quantity of boron on the floor and protruding from the air boot around the "A" loop } \\
\text { RCS hot leg pipe. Ultrasonic testing (UT) and eddy current testing (ET) identified an axial crack-like } \\
\text { indication approximately } 2.7 \text { inches long located approximately } 7 \text { degrees counterclockwise from top } \\
\text { dead center of the first weld between the reactor vessel nozzle and the "A" loop hot leg piping } \\
\text { approximately } 3 \text { feet from the reactor vessel. Based on the UT data, the axial crack-like indication } \\
\text { began at the inner diameter and shows evidence of complete through-wall extension. Visual } \\
\text { examination from the outer diameter identified a small "weep hole" in the center of the weld at } \\
\text { approximately the same circumferential location as the UT and ET indications. }\end{array}$ & Piping \\
\hline IN & 2000-09 & $\begin{array}{l}\text { Steam } \\
\text { Generator } \\
\text { Tube } \\
\text { Failure at }\end{array}$ & $\begin{array}{l}\text { The U.S. Nuclear Regulatory Commission is issuing this information notice to inform addressees of a } \\
\text { steam generator tube failure at Indian Point Unit } 2 \text {. NRC investigations of the licensee's steam } \\
\text { generator inspection program are ongoing and any potentially generic issues identified will be } \\
\text { communicated in a separate generic communication. However, the investigations to date re-emphasize }\end{array}$ & $\begin{array}{l}\text { Steam } \\
\text { Generator }\end{array}$ \\
\hline
\end{tabular}




\begin{tabular}{|c|c|c|c|c|}
\hline Type & No. & Title & Topic & $\begin{array}{c}\text { SPC } \\
\text { Affected }\end{array}$ \\
\hline & & $\begin{array}{l}\text { Indian } \\
\text { Point Unit } \\
2\end{array}$ & $\begin{array}{l}\text { the importance of licensee involvement with ongoing industry efforts to understand and detect steam } \\
\text { generator degradation. It is expected that recipients will review the information for applicability to } \\
\text { their facilities and consider actions, as appropriate, to avoid similar problems. However, suggestions } \\
\text { contained in this information notice are not NRC requirements; therefore, no specific action or written } \\
\text { response is required. } \\
\text { Discussion } \\
\text { On February } 15,2000 \text {, at } 7: 17 \text { p.m., the Indian Point Unit } 2 \text { nuclear plant experienced a steam } \\
\text { generator tube failure, which required the declaration of an Alert at } 7: 29 \text { p.m., and a manual reactor } \\
\text { trip at } 7: 30 \text { p.m. The operators identified that the } \# 24 \text { steam generator was the source of the leak and } \\
\text { completed isolation of the } \# 24 \text { steam generator by } 8: 31 \text { p.m. }\end{array}$ & \\
\hline IN & $\begin{array}{l}\text { 99-10, } \\
\text { Rev. 1, } \\
\text { and 99- } \\
10\end{array}$ & $\begin{array}{l}\text { Degradatio } \\
\mathrm{n} \quad \text { of } \\
\text { Prestressin } \\
\mathrm{g} \text { Tendon } \\
\text { Systems in } \\
\text { Prestressed } \\
\text { Concrete } \\
\text { Containme } \\
\text { nts }\end{array}$ & $\begin{array}{l}\text { The U.S. Nuclear Regulatory Commission (NRC) is issuing this information notice to alert addressees } \\
\text { to the degradation of prestressing systems components of prestressed concrete containments (PCCs). } \\
\text { The following specific items are addressed: (1) breakage of prestressing tendon wires, (2) effects of } \\
\text { high temperature on the prestressing forces in tendons, and (3) trend analysis of prestressing forces. It } \\
\text { is expected that recipients will review the information for applicability to their facilities and consider } \\
\text { actions, as appropriate, to avoid similar problems. However, suggestions contained in this information } \\
\text { notice are not NRC requirements; therefore, no specific action or written response is required. } \\
\text { Discussion } \\
\text { On April 13, 1999, the staff issued Information Notice 99-10 to describe the degradation associated } \\
\text { with the tendon prestressing system of prestressed concrete containments (PCCs). The staff received a } \\
\text { letter from Duke Energy on May 6, 1999, which indicated that Attachment } 3 \text { to IN 99-10, } \\
\text { "Comparison and Trending of Prestressing Forces," misrepresented the Oconee experience. This } \\
\text { revision corrects the observations made by the staff concerning the sixth tendon surveillance } \\
\text { performed at Oconee Unit } 3 \text { in the summer of } 1995 \text { and provides other editorial and clarifying } \\
\text { changes. } \\
\text { Inspections of PCCs and PCC tendons have identified a number of concerns related to the degradation } \\
\text { of prestressing tendon systems in PCCs. Findings relevant to these concerns are: Breakage of } \\
\text { Prestressing Tendon Wires, Effects of High Temperature on the Prestressing Forces in Tendons, } \\
\text { Comparison and Trending of Prestressing Forces }\end{array}$ & $\begin{array}{l}\text { Prestressing } \\
\text { tendons }\end{array}$ \\
\hline IN & $98-45$ & $\begin{array}{l}\text { Cavitation } \\
\text { Erosion of }\end{array}$ & $\begin{array}{l}\text { The U.S. Nuclear Regulatory Commission (NRC) is issuing this information notice to alert addressees } \\
\text { to potential problems caused by cavitation erosion of letdown line orifices in the chemical and volume }\end{array}$ & Piping \\
\hline
\end{tabular}




\begin{tabular}{|c|c|c|c|c|}
\hline Туре & No. & Title & Topic & $\begin{array}{c}\text { SPC } \\
\text { Affected }\end{array}$ \\
\hline & & $\begin{array}{l}\text { Letdown } \\
\text { Line } \\
\text { Orifices } \\
\text { Resulting } \\
\text { in Fatigue } \\
\text { Cracking of } \\
\text { Pipe Welds }\end{array}$ & $\begin{array}{l}\text { control system (CVCS). Such erosion has contributed to failures in pipe welds downstream of the } \\
\text { letdown line orifices. It is expected that recipients will review the information for applicability to their } \\
\text { facilities and consider actions, as appropriate, to avoid similar problems. However, suggestions } \\
\text { contained in this information notice are not NRC requirements; therefore, no specific action or written } \\
\text { response is required. } \\
\text { Discussion } \\
\text { On September 11, 1996, Surry Power Station, Unit } 2 \text {, experienced its fourth socket weld failure in } 12 \\
\text { months. The failed welds were located on the low-pressure portion of the CVCS letdown line, just } \\
\text { downstream of the pressure-reducing orifice isolation valves. The licensee determined that the most } \\
\text { likely cause of the weld failure was flow-induced vibration. The licensee performed a microscopic } \\
\text { examination of the Unit } 2 \text { letdown line orifices and concluded that two of the orifices exhibited cone- } \\
\text { shaped patterns, wider at the discharge of the orifice and tapering toward the inlet of the orifice. In } \\
\text { addition, the orifice exhibited very rough and irregular surface profiles. The damage to the letdown } \\
\text { line orifices is indicative of cavitation erosion and is believed to have contributed to flow-induced } \\
\text { vibration of the letdown line and to the socket weld failures. } \\
\text { On March 15, 1997, the licensee performed radiographic examinations on the Surry Unit } 1 \text { letdown } \\
\text { line orifices to check for an erosion condition similar to that previously seen in the Unit } 2 \text { letdown line } \\
\text { orifices. The licensee concluded that erosion was present in all three orifices and that the most } \\
\text { extensive deterioration was present in the } 45 \text {-gpm orifice. } \\
\text { Socket welds have also failed at Diablo Canyon Nuclear Power Plant, Unit } 2 \text {. From June } 1989 \text { through } \\
\text { December } 1990 \text {, four socket welds failed on two of three reactor coolant system (RCS) pressure } \\
\text { letdown lines in the CVCS. All four failed welds were located on the piping downstream of the RCS } \\
\text { letdown orifice isolation valves. On March } 19 \text {, 1991, Diablo Canyon Unit } 2 \text { experienced the fifth } \\
\text { letdown leak since June } 1989 \text {. During the repair effort, the licensee determined that the cause of the } \\
\text { failures was flow-induced vibration due to a damaged letdown orifice upstream of the weld failure. }\end{array}$ & \\
\hline IN & $98-27$ & $\begin{array}{l}\text { Steam } \\
\text { Generator } \\
\text { Tube End } \\
\text { Cracking }\end{array}$ & $\begin{array}{l}\text { The U.S. Nuclear Regulatory Commission (NRC) is issuing this information notice to inform licensees } \\
\text { of instances of steam generator tube-end cracking. It is expected that recipients will review the } \\
\text { information for applicability to their facilities and consider actions, as appropriate, to avoid similar } \\
\text { problems. However, suggestions contained in this information notice are not NRC requirements; } \\
\text { therefore, no specific action or written response in required. } \\
\text { Discussion }\end{array}$ & $\begin{array}{l}\text { Steam } \\
\text { Generators }\end{array}$ \\
\hline
\end{tabular}




\begin{tabular}{|c|c|c|c|c|}
\hline Type & No. & Title & Topic & $\begin{array}{c}\text { SPC } \\
\text { Affected }\end{array}$ \\
\hline & & & $\begin{array}{l}\text { Entergy, the licensee for Arkansas Nuclear One, Unit } 1 \text { (ANO-1), conducted leak testing on each once- } \\
\text { through steam generator (OTSG) during the spring } 1998 \text { refueling outage to identify the source of } \\
\text { primary-to-secondary leakage measured during the previous operating cycle. The leak testing revealed } \\
\text { a small leak in one tube of each OTSG. The leak in each tube was in flaws in the tube hardroll just } \\
\text { below the upper tubesheet seal weld. Subsequent eddy current inspections identified primary-water } \\
\text { stress-corrosion cracking (PWSCC) in each of the two tubes. The through-wall flaws in the tubes were } \\
\text { oriented both axially and circumferentially in the tubes. Examinations of all upper tubesheet seal weld } \\
\text { areas in both OTSGs revealed possible flaws in } 1896 \text { additional tubes. } \\
\text { Eddy current inspections completed at Davis-Besse Nuclear Power Station in April } 1998 \text { identified } \\
\text { five tubes with "tube end anomalies" believed to be related to the flaws identified at ANO-1. Duke } \\
\text { Power Co. recently completed an assessment to determine if its Babcock and Wilcox-designed, } \\
\text { operating units, Oconee Nuclear Station, Units } 1 \text { and 3, were affected by tube-end cracking. The } \\
\text { licensee concluded that } 372 \text { indications in the Unit } 1 \text { OTSGs and } 61 \text { indications in the Unit } 3 \text { OTSGs } \\
\text { exceeded the repair criteria in the Oconee Technical Specifications. Tube-end cracking has also been } \\
\text { reported for the steam generators at Prairie Island Nuclear Generating Plant. }\end{array}$ & \\
\hline IN & $98-26$ & $\begin{array}{l}\text { Settlement } \\
\text { Monitoring } \\
\text { and } \\
\text { Inspection } \\
\text { of Plant } \\
\text { Structures } \\
\text { Affected by } \\
\text { Degradatio } \\
\text { n of Porous } \\
\text { Concrete } \\
\text { Subfoundat } \\
\text { ions }\end{array}$ & $\begin{array}{l}\text { The U.S. Nuclear Regulatory Commission (NRC) is issuing this information notice (IN) to inform } \\
\text { addressees who own and operate facilities with plant sites that include structures with porous concrete } \\
\text { subfoundations of the possibility of degradation of these subfoundations. Such degradation could have } \\
\text { deleterious effects on structures, systems, and components (SSCs). } \\
\text { It is expected that recipients will review the information for applicability to their facilities and consider } \\
\text { actions, as appropriate, to avoid similar problems. However, suggestions contained in this information } \\
\text { notice are not NRC requirements; therefore, no specific action or written response is required. } \\
\text { Discussion } \\
\text { The containment structure at Millstone Nuclear Power Station, Unit } 3 \text { (MNPS-3), has a 3.05-meter } \\
\text { (10-foot) thick reinforced-concrete basemat founded on rock. Between the foundation rock surface and } \\
\text { the underside of the basemat are several layers of different materials. In the upper porous concrete } \\
\text { layer, } 15 \text {-cm (6-inch) diameter porous concrete pipes are installed to collect and drain ground water, } \\
\text { which may seep down along the periphery of the containment wall. The collected water drains into } \\
\text { two sumps inside the engineered safety features (ESF) building. } \\
\text { The MNPS-3 licensee, Northeast Nuclear Energy Company, identified the issue of cement erosion } \\
\text { from the porous concrete drainage system in } 1987 \text { upon examination of the accumulated white residue }\end{array}$ & $\begin{array}{l}\text { Concrete } \\
\text { porous } \\
\text { concrete } \\
\text { subfoundatio } \\
\mathrm{n}\end{array}$ \\
\hline
\end{tabular}




\begin{tabular}{|c|c|c|c|c|}
\hline Type & No. & Title & Topic & $\begin{array}{c}\text { SPC } \\
\text { Affected } \\
\end{array}$ \\
\hline & & & $\begin{array}{l}\text { in the two lower drain sumps in the ESF building. IN 97-11, "Cement Erosion From Containment } \\
\text { Subfoundations at Nuclear Power Plants," was issued on March 21, 1997, to alert addressees to the } \\
\text { potential for erosion of cement from porous concrete subfoundations. }\end{array}$ & \\
\hline IN & $98-11$ & $\begin{array}{l}\text { Cracking of } \\
\text { Reactor } \\
\text { Vessel } \\
\text { Internal } \\
\text { Baffle } \\
\text { Former } \\
\text { Bolts in } \\
\text { Foreign } \\
\text { Plants }\end{array}$ & $\begin{array}{l}\text { The U.S. Nuclear Regulatory Commission (NRC) is issuing this information notice to alert addressees } \\
\text { to the cracking of reactor vessel internal baffle former bolts found at several foreign PWRs and to } \\
\text { inform addressees of actions taken and planned by domestic PWR owners groups in response to this } \\
\text { experience. It is expected that the recipients will review the information for applicability to their } \\
\text { facilities and consider actions, as appropriate, to avoid similar problems. However, suggestions } \\
\text { contained in this information notice are not NRC requirements; IN 98-11 } \\
\text { Discussion } \\
\text { European plants identified the cracking of baffle former bolts as early as } 1988 \text { and this problem } \\
\text { continues to occur. Although this cracking is not fully understood, testing of cracked bolts suggests an } \\
\text { age-related intergranular stress-corrosion cracking process influenced by bolt material, fluence, stress, } \\
\text { and temperature. The reported cracking occurred in } 316 \text { cold-worked stainless steel bolts. Most of the } \\
\text { cracking reported has been in four French } 900-\mathrm{MWe} \text { (megawatt electric) PWRs. }\end{array}$ & $\begin{array}{l}\text { RPV reactor } \\
\text { internals - } \\
\text { internal } \\
\text { baffle bolts }\end{array}$ \\
\hline $\begin{array}{l}\text { Bulletin } \\
\text { (BL) }\end{array}$ & $2004-01$ & $\begin{array}{l}\text { Inspection } \\
\text { of Alloy } \\
82 / 182 / 600 \\
\text { Materials } \\
\text { Used in the } \\
\text { Fabrication } \\
\text { of } \\
\text { Pressurizer } \\
\text { Penetration } \\
\text { s and } \\
\text { Steam } \\
\text { Space } \\
\text { Piping } \\
\text { Connection } \\
\text { S } \\
\text { Pressurized } \\
\text {-Water } \\
\text { Reactors }\end{array}$ & $\begin{array}{l}\text { The U.S. Nuclear Regulatory Commission (NRC) is issuing this bulletin to: } \\
\text { (1) advise PWR licensees that current methods of inspecting Alloy } 82 / 182 / 600 \text { materials used in the } \\
\text { fabrication of pressurizer penetrations and steam space piping connections may need to be } \\
\text { supplemented with additional measures to detect and adequately characterize flaws due to primary } \\
\text { water stress corrosion cracking (PWSCC), } \\
\text { (2) request PWR addressees to provide the NRC with information related to the materials from which } \\
\text { the pressurizer penetrations and steam space piping connections at their facilities were fabricated, } \\
\text { (3) request PWR licensees to provide the NRC with information related to the inspections that have } \\
\text { been and those that will be performed to ensure that degradation of Alloy } 82 / 182 / 600 \text { materials used in } \\
\text { the fabrication of pressurizer penetrations and steam space piping connections will be identified, } \\
\text { adequately characterized, and repaired, and } \\
\text { (4) require PWR addresses to provide a written response to the NRC in accordance with the provisions } \\
\text { of Section 50.54(f) of Title } 10 \text { of the Code of Federal Regulations (10 CFR 50.54(f)). } \\
\text { Operating experience, both domestic and foreign, has demonstrated that Alloy } 82 / 182 / 600 \text { materials } \\
\text { connected to a PWR's pressurizer may be particularly susceptible to PWSCC. Since the late } 1980 \text { 's, } \\
\text { approximately } 50 \text { Alloy } 600 \text { pressurizer heater sleeves at Combustion Engineering-designed (CE- } \\
\text { designed) facilities in the United States have shown evidence of RCPB leakage which has been } \\
\text { attributed to PWSCC. The most recent events of this type }\end{array}$ & $\begin{array}{l}\text { Pressurizer } \\
\text { and Piping }\end{array}$ \\
\hline
\end{tabular}




\begin{tabular}{|c|c|c|c|c|}
\hline Type & No. & Title & Topic & $\begin{array}{c}\text { SPC } \\
\text { Affected } \\
\end{array}$ \\
\hline & & & $\begin{array}{l}\text { occurred at Millstone, Unit 2, and Waterford, Unit 3, in October 2003, and at Palo Verde, Unit 3, in } \\
\text { February 2004. All available evidence from finite element modeling studies and limited } \\
\text { nondestructive evaluation (NDE) has suggested that these leakage events were the result of } \\
\text { axially-oriented PWSCC of the pressure boundary portion of these heater sleeves. However, } \\
\text { NDE results from Palo Verde, Unit 2's fall } 2003 \text { refueling outage, on heater sleeves which had not } \\
\text { shown evidence of leakage have demonstrated that circumferentially-oriented PWSCC can occur in } \\
\text { the non-pressure boundary portion (i.e., above the J-groove attachment weld) of these components. } \\
\text { Cracking in a TMI-1 diaphragm plate was attributed to PWSCC in the heat affected zone of the seal } \\
\text { weld. Boric acid corrosion of the low alloy steel strongback was also observed to have resulted from } \\
\text { the leakage. } \\
\text { Related Generic Communications } \\
\text { - Bulletin 2003-02, "Leakage From Reactor Pressure Vessel Lower Head Penetrations and Reactor } \\
\text { Coolant Pressure Boundary Integrity," August 21, 2003 (ADAMS Accession No. ML032320153) BL } \\
\text { 2004-01 } \\
\text {-Bulletin 2002-02, "Reactor Pressure Vessel Head and Vessel Head Penetration Nozzle Inspection } \\
\text { Programs," August 9, 2002 (ADAMS Accession No. ML022200494) } \\
\text {-Bulletin 2002-01, "Reactor Pressure Vessel Head Degradation and Reactor Coolant Pressure } \\
\text { Boundary Integrity," March 18, 2002 (ADAMS Accession No. ML020770497) } \\
\text {-Bulletin 2001-01, "Circumferential Cracking of Reactor Pressure Vessel Head Penetration Nozzles," } \\
\text { August 3, 2001 (ADAMS Accession No. ML012080284) }\end{array}$ & \\
\hline $\mathrm{BL}$ & $2003-02$ & 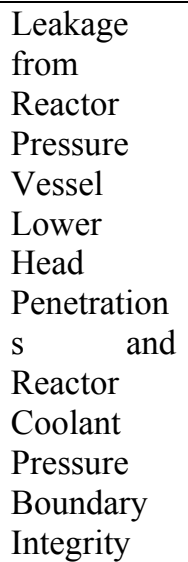 & $\begin{array}{l}\text { The U.S. Nuclear Regulatory Commission (NRC) is issuing this bulletin to: } \\
\text { (1) advise PWR addressees that current methods of inspecting the RPV lower heads may } \\
\text { need to be supplemented with additional measures (e.g., bare-metal visual inspections) } \\
\text { to detect reactor coolant pressure boundary (RCPB) leakage, } \\
\text { (2) request PWR addressees to provide the NRC with information related to inspections } \\
\text { that have been or will be performed to verify the integrity of the RPV lower head } \\
\text { penetrations, and } \\
\text { (3) require PWR addresses to provide a written response to the NRC in accordance with } \\
\text { the provisions of Section 50.54(f) of Title } 10 \text { of the Code of Federal Regulations } \\
\text { (10 CFR 50.54(f)). } \\
\text { The RPV and its head penetrations are an integral part of the RCPB, and their integrity is } \\
\text { important to the safe operation of the plant. The recent identification of cracking and leakage } \\
\text { from two BMI penetrations at South Texas Project Unit } 1 \text { (STP Unit 1) raises questions about potential }\end{array}$ & $\begin{array}{l}\text { RPV head } \\
\text { penetrations }\end{array}$ \\
\hline
\end{tabular}




\begin{tabular}{|c|c|c|c|c|}
\hline Type & No. & Title & Topic & $\begin{array}{c}\text { SPC } \\
\text { Affected }\end{array}$ \\
\hline & & & $\begin{array}{l}\text { degradation mechanisms which may be active in this area. In addition, licensee responses to the } \\
\text { Bulletin 2002-01 followup RAIs raised questions about the adequacy of inspections performed by } \\
\text { licensees to detect leakage from RPV lower head penetrations. } \\
\text { Related Generic Communications } \\
\text { - Regulatory Issue Summary 2003-13, "NRC Review of Responses to Bulletin 2002-01, 'Reactor } \\
\text { Pressure Vessel Head Degradation and Reactor Coolant Pressure Boundary Integrity,' July 29, } 2003 \\
\text { (ADAMS Accession No. ML032100653) } \\
\text { - Information Notice 2003-11 "Leakage Found on Bottom-Mounted Instrumentation Nozzles," August } \\
\text { 13, } 2003 \text { (ADAMS Accession No. ML032250135) } \\
\text { - Bulletin 2002-02, "Reactor Pressure Vessel Head and Vessel Head Penetration Nozzle Inspection } \\
\text { Programs," August 9, 2002 (ADAMS Accession No. ML022200494) } \\
\text { - Bulletin 2002-01, "Reactor Pressure Vessel Head Degradation and Reactor Coolant Pressure } \\
\text { Boundary Integrity," March 18, 2002 (ADAMS Accession No. ML020770497) } \\
\text { - Generic Letter 88-05, "Boric Acid Corrosion of Carbon Steel Reactor Pressure Boundary } \\
\text { - Components in PWR Plants," March 17, 1988 (ADAMS Accession No. ML031130424) }\end{array}$ & \\
\hline $\mathrm{BL}$ & 2003-01 & $\begin{array}{l}\text { Potential } \\
\text { Impact of } \\
\text { Debris } \\
\text { Blockage } \\
\text { on } \\
\text { Emergency } \\
\text { Sump at } \\
\text { Recirculati } \\
\text { on a } \\
\text { Pressurized } \\
\text {-Water } \\
\text { Reactors }\end{array}$ & $\begin{array}{l}\text { The U.S. Nuclear Regulatory Commission (NRC) is issuing this bulletin to: } \\
\text { (1) Inform addressees of the results of NRC-sponsored research identifying the potential } \\
\text { susceptibility of pressurized-water reactor (PWR) recirculation sump screens to debris } \\
\text { blockage in the event of a high-energy line break (HELB) requiring recirculation } \\
\text { operation of the emergency core cooling system (ECCS) or containment spray system } \\
\text { (CSS). } \\
\text { (2) Inform addressees of the potential for additional adverse effects due to debris blockage } \\
\text { of flowpaths necessary for ECCS and CSS recirculation and containment drainage. } \\
\text { (3) Request that, in light of these potentially adverse effects, addressees confirm their } \\
\text { compliance with } 10 \text { CFR 50.46(b)(5) and other existing applicable regulatory } \\
\text { requirements, or describe any compensatory measures implemented to reduce the } \\
\text { potential risk due to post-accident debris blockage as evaluations to determine } \\
\text { compliance proceed. } \\
\text { (4) Require addressees to provide the NRC a written response in accordance with } \\
\text { 10 CFR 50.54(f). } \\
\text { In the event of a HELB within the containment of a PWR, energetic pressure waves and fluid } \\
\text { jets would impinge upon materials in the vicinity of the break, such as thermal insulation, } \\
\text { coatings, and concrete, causing damage and generating debris. Debris could also be }\end{array}$ & $\begin{array}{l}\text { ECCS and } \\
\text { CSS sump \& } \\
\text { drainage } \\
\text { blockage }\end{array}$ \\
\hline
\end{tabular}




\begin{tabular}{|c|c|c|c|c|}
\hline Type & No. & Title & Topic & $\begin{array}{c}\text { SPC } \\
\text { Affected }\end{array}$ \\
\hline & & & 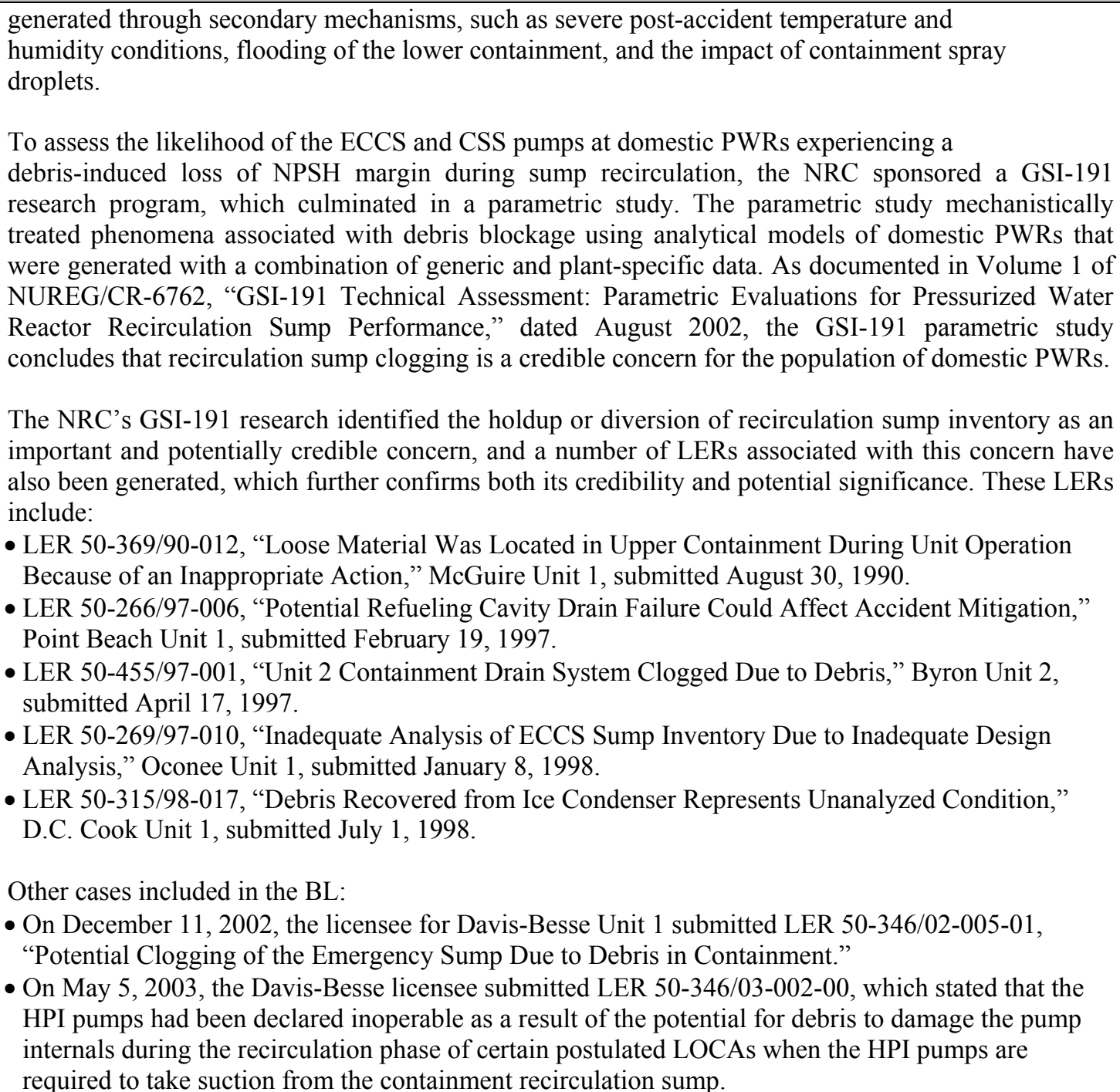 & \\
\hline
\end{tabular}




\begin{tabular}{|c|c|c|c|c|}
\hline Type & No. & Title & Topic & $\begin{array}{c}\text { SPC } \\
\text { Affected }\end{array}$ \\
\hline & & & $\frac{\text { Related Generic Communications }}{24 \text { Generic Communications listed related to ECCS and CSS sump and strainer blockage. }}$ & \\
\hline $\mathrm{BL}$ & $2002-02$ & $\begin{array}{l}\text { Reactor } \\
\text { Pressure } \\
\text { Vessel } \\
\text { Head and } \\
\text { Vessel } \\
\text { Head } \\
\text { Penetration } \\
\text { Nozzle } \\
\text { Inspection } \\
\text { Programs }\end{array}$ & $\begin{array}{l}\text { The U.S. Nuclear Regulatory Commission (NRC) is issuing this bulletin to: } \\
\text { (1) Advise pressurized-water reactor (PWR) addressees that visual examinations, as a } \\
\text { primary inspection method for the reactor pressure vessel (RPV) head and vessel head } \\
\text { penetration (VHP) nozzles, may need to be supplemented with additional measures } \\
\text { (e.g., volumetric and surface examinations) to demonstrate compliance with applicable } \\
\text { regulations. } \\
\text { (2) Advise PWR addressees that inspection methods and frequencies to demonstrate } \\
\text { compliance with applicable regulations should be demonstrated to be reliable and } \\
\text { effective. } \\
\text { (3) Request information from all PWR addressees concerning their RPV head and VHP } \\
\text { nozzle inspection programs to ensure compliance with applicable regulatory } \\
\text { requirements. } \\
\text { (4) Require all PWR addressees to provide written responses to this bulletin related to their } \\
\text { inspection program plans. } \\
\text { Discussion } \\
\text { Primary water stress corrosion cracking (PWSCC) in PWR control rod drive mechanism } \\
\text { (CRDM) nozzles and other vessel head penetration nozzles fabricated from Alloy } 600 \text { is not a } \\
\text { new issue; axial cracking in the CRDM nozzles has been identified since the late } 1980 \text { s. In } \\
\text { addition, numerous small-bore Alloy } 600 \text { nozzles and pressurizer heater sleeves have } \\
\text { experienced leaks attributable to PWSCC. The area of interest for potential cracking of RPV head } \\
\text { penetrations is the pressure-retaining boundary, which includes the J-groove weld } \\
\text { between the nozzle and reactor vessel head and the portion of the nozzle inside the head. } \\
\text { Inspections of the reactor nozzles at Oconee Nuclear Stations } 2 \text { and } 3 \text { in early } 2001 \text { identified } \\
\text { circumferential cracking of the nozzles above the J-groove weld. } \\
\text { In early March 2002, while conducting VHP nozzle inspections that were prompted by NRC } \\
\text { Bulletin 2001-01, Davis-Besse Nuclear Power Station identified a large cavity in the RPV head near } \\
\text { the top of the dome. The cavity was adjacent to a nozzle which was leaking as a result of through-wall } \\
\text { axial cracking, and was located in an area of the RPV head that the licensee had left covered with boric } \\
\text { acid deposits for a number of years. }\end{array}$ & $\begin{array}{l}\text { RPV head \& } \\
\text { nozzle } \\
\text { penetrations }\end{array}$ \\
\hline
\end{tabular}




\begin{tabular}{|c|c|c|c|c|}
\hline Type & No. & Title & Topic & $\begin{array}{c}\text { SPC } \\
\text { Affected } \\
\end{array}$ \\
\hline & & & $\begin{array}{l}\text { Related Generic Communications } \\
\text { - Bulletin 2002-01, "Reactor Pressure Vessel Head Degradation and Reactor Coolant Pressure } \\
\text { Boundary Integrity," March 18, 2002. [ADAMS Accession No. ML020770497] } \\
\text { - Information Notice 2002-11, "Recent Experience with Degradation of Reactor Pressure Vessel } \\
\text { Head,” March 12, 2002. [ADAMS Accession No. ML020700556] } \\
\text { - Bulletin 2001-01, "Circumferential Cracking of Reactor Pressure Vessel Head Penetration Nozzles," } \\
\text { August 3, 2001. [ADAMS Accession No. ML012080284] } \\
\text { - Information Notice 2001-05, "Through-Wall Circumferential Cracking of Reactor Pressure Vessel } \\
\text { Head Control Rod Drive Mechanism Penetration Nozzles at Oconee Nuclear Station, Unit 3," April } \\
\text { 30, 2001. [ADAMS Accession No. ML011160588] } \\
\text { - Generic Letter 97-01, "Degradation of Control Rod Drive Mechanism Nozzle and Other Vessel } \\
\text { Closure Head Penetrations," April 1, 1997. } \\
\text { - Information Notice 96-11, "Ingress of Demineralizer Resins Increases Potential for Stress Corrosion } \\
\text { Cracking of Control Rod Drive Mechanism Penetrations,” February 14, 1996. } \\
\text { - Information Notice 90-10, "Primary Water Stress Corrosion Cracking of INCONEL 600," February } \\
\text { 23, 1990. } \\
\text { - Generic Letter 88-05, "Boric Acid Corrosion of Carbon Steel Reactor Pressure Boundary } \\
\text { Components in PWR Plants,” March 17, 1988. } \\
\text { - NUREG/CR-6245,"Assessment of Pressurized Water Reactor Control Rod Drive Mechanism } \\
\text { Nozzle Cracking," October 1994. }\end{array}$ & \\
\hline $\mathrm{BL}$ & $2002-01$ & $\begin{array}{l}\text { Reactor } \\
\text { Pressure } \\
\text { Vessel } \\
\text { Head } \\
\text { Degradatio } \\
\text { n and } \\
\text { Reactor } \\
\text { Coolant } \\
\text { Pressure } \\
\text { Boundary } \\
\text { Integrity }\end{array}$ & $\begin{array}{l}\text { The U.S. Nuclear Regulatory Commission (NRC) is issuing this bulletin to require pressurized-water } \\
\text { reactor (PWR) addressees to submit: } \\
\text { (1) information related to the integrity of the reactor coolant pressure boundary including the reactor } \\
\text { pressure vessel head and the extent to which inspections have been undertaken to satisfy applicable } \\
\text { regulatory requirements, and } \\
\text { (2) the basis for concluding that plants satisfy applicable regulatory requirements related to the } \\
\text { structural integrity of the reactor coolant pressure boundary and future inspections will ensure } \\
\text { continued compliance with applicable regulatory requirements, and } \\
\text { (3) a written response to the NRC in accordance with the provisions of Title } 10 \text {, Section } 50.54(\mathrm{f}) \text {, of } \\
\text { the Code of Federal Regulations (10 CFR 50.54(f)) if they are unable to provide the information or } \\
\text { they can not meet the requested completion dates. } \\
\text { Discussion }\end{array}$ & $\begin{array}{l}\text { RPV head \& } \\
\text { nozzle } \\
\text { penetrations }\end{array}$ \\
\hline
\end{tabular}




\begin{tabular}{|c|c|c|c|c|}
\hline Type & No. & Title & Topic & $\begin{array}{c}\text { SPC } \\
\text { Affected } \\
\end{array}$ \\
\hline & & & $\begin{array}{l}\text { On August 3, 2001, the NRC issued Bulletin 2001-01, "Circumferential Cracking of Reactor Pressure } \\
\text { Vessel Head Penetration Nozzles" (ADAMS Accession Number ML012080284). That bulletin } \\
\text { described instances of cracked and leaking Alloy } 600 \text { reactor pressure vessel head penetration nozzles, } \\
\text { including control rod drive mechanism and thermocouple nozzles. In response to that bulletin, } \\
\text { pressurized-water reactor licensees provided their plans for inspecting their reactor pressure vessel } \\
\text { head penetrations and/or the outside surface of the reactor pressure vessel head to determine whether } \\
\text { the nozzles were leaking. Some plants have completed these inspections. In conducting these } \\
\text { inspections at the Davis-Besse Nuclear Power Station in February and March 2002, the licensee } \\
\text { identified three control rod drive mechanism nozzles with indications of axial cracking that resulted in } \\
\text { reactor coolant pressure boundary leakage. } \\
\underline{\text { Related Generic Communications }}\end{array}$ & \\
\hline BL & $2001-01$ & $\begin{array}{l}\text { Circumfere } \\
\text { ntial } \\
\text { Cracking of } \\
\text { Reactor } \\
\text { Pressure } \\
\text { Vessel } \\
\text { Head } \\
\text { Penetration } \\
\text { Nozzles }\end{array}$ & $\begin{array}{l}\text { The U.S. Nuclear Regulatory Commission (NRC) is issuing this bulletin to: } \\
\text { (1) request that addressees provide information related to the structural integrity of the reactor pressure } \\
\text { vessel head penetration (VHP) nozzles for their respective facilities, including the extent of VHP } \\
\text { nozzle leakage and cracking that has been found to date, the inspections and repairs that have been } \\
\text { undertaken to satisfy applicable regulatory requirements, and the basis for concluding that their plans } \\
\text { for future inspections will ensure compliance with applicable regulatory requirements, and } \\
\text { (2) require that all addressees provide to the NRC a written response in accordance with the provisions } \\
\text { of } 10 \text { CFR 50.54(f). } \\
\text { Discussion } \\
\text { The recent discoveries of cracked and leaking Alloy } 600 \text { VHP nozzles, including control rod drive } \\
\text { mechanism (CRDM) and thermocouple nozzles, at four pressurized water reactors (PWRs) have raised } \\
\text { concerns about the structural integrity of VHP nozzles throughout the PWR industry. Nozzle cracking } \\
\text { at Oconee Nuclear Station Unit } 1 \text { (ONS1) in November } 2000 \text { and Arkansas Nuclear One Unit } 1 \\
\text { (ANO1) in February } 2001 \text { was limited to axial cracking, an occurrence deemed to be of limited safety } \\
\text { concern in the NRC staff's generic safety evaluation on the cracking of VHP nozzles, dated November } \\
\text { 19, 1993. However, the discovery of circumferential cracking at Oconee Nuclear Station Unit } 3 \\
\text { (ONS3) in February } 2001 \text { and Oconee Nuclear Station Unit } 2 \text { (ONS2) in April } 2001 \text { - particularly the } \\
\text { large circumferential cracking identified in two CRDM nozzles at ONS3 - has raised concerns about } \\
\text { the potential safety implications and prevalence of cracking in VHP nozzles in PWRs. } \\
\text { Related Generic Communications }\end{array}$ & $\begin{array}{l}\text { RPV head } \\
\text { penetrations }\end{array}$ \\
\hline
\end{tabular}




\begin{tabular}{|c|c|c|c|c|}
\hline Type & No. & Title & Topic & SPC Affected \\
\hline & & & Same as BL 2002-02. & \\
\hline
\end{tabular}


Table 4-2 Summary of Degradation Information by Generic Communications

\begin{tabular}{|c|c|c|}
\hline $\begin{array}{c}\text { Generic } \\
\text { Correspondence }\end{array}$ & Component / Subcomponent & $\begin{array}{l}\text { Number of Generic } \\
\text { Correspondences on } \\
\text { this Component }\end{array}$ \\
\hline \multirow[t]{4}{*}{ Generic Letter } & Steam Generators & 4 \\
\hline & Strainer - Sump Pump Screens & 2 \\
\hline & RPV - CRD nozzles \& other penetrations & 1 \\
\hline & & Subtotal $=7$ \\
\hline \multirow[t]{4}{*}{ Bulletin } & RPV - head penetrations, \& coolant boundary & 4 \\
\hline & Strainer - Sump Pump Screens & 1 \\
\hline & Pressurizer \& steam space piping connection & 1 \\
\hline & & Subtotal $=6$ \\
\hline \multirow[t]{10}{*}{ Information Notice } & Steam Generators & 9 \\
\hline & RPV - nozzles & 8 \\
\hline & Piping & 7 \\
\hline & Containment & 3 \\
\hline & Concrete & 2 \\
\hline & Pressurizer & 2 \\
\hline & Spent fuel pool - liner & 1 \\
\hline & Equipment - boric acide leakage onto & 1 \\
\hline & & Subtotal $=33$ \\
\hline & & Total $=46$ \\
\hline
\end{tabular}


Table 4-3 Summary of Degradation by Components

\begin{tabular}{|c|c|}
\hline Component & $\begin{array}{c}\text { Number of Generic } \\
\text { Correspondences }\end{array}$ \\
\hline Steam Generators & 13 \\
\hline RPV & 13 \\
\hline Piping & 7 \\
\hline Strainer - Sump Pump Screens & 3 \\
\hline Pressurizer & 3 \\
\hline Containment & 3 \\
\hline Concrete & 2 \\
\hline Spent Fuel Pool & 1 \\
\hline Equipment - boric acide leakage onto & 1 \\
\hline & Total $=46$ \\
\hline
\end{tabular}


Table 4-4 License Renewal Applications for Information Related to Aging Degradation - Palisades Nuclear Power Plant [Available on the NRC Website]

\begin{tabular}{|c|c|c|}
\hline $\begin{array}{l}\text { Aging } \\
\text { Management } \\
\text { Program }\end{array}$ & $\begin{array}{c}\text { Description of Aging Management Program } \\
\text { (AMP) }\end{array}$ & Operating Experience \\
\hline $\begin{array}{ll}\text { Alloy } 600 \\
\text { Program }\end{array}$ & $\begin{array}{l}\text { This is an existing program that manages aging due } \\
\text { to PWSCC of the Primary Coolant System (PCS) } \\
\text { pressure boundary Alloy } 600 \text { components, including } \\
\text { Inconel } 82 / 182 \text { weld joints, reactor vessel head } \\
\text { penetrations, etc. }\end{array}$ & $\begin{array}{l}\text { Industry: } \\
\text { Instances of degradation of material have occurred as a result of PWSCC } \\
\text { Plant Specific: } \\
\text { - Pressurizer Temperature Element Penetration } \\
\text { - Pressurizer Safe End } \\
\text { - CRD Nozzle penetration indications (2) }\end{array}$ \\
\hline $\begin{array}{l}\text { ASME } \\
\text { Section XI } \\
\text { IWB, IWC, } \\
\text { IWD, IWF } \\
\text { Inservice } \\
\text { Inspection } \\
\text { Program }\end{array}$ & $\begin{array}{l}\text { This is an existing program that facilitates } \\
\text { inspections to identify and correct degradation in } \\
\text { Class 1, 2, and } 3 \text { piping, components, their supports } \\
\text { and integral attachments. The program includes } \\
\text { periodic visual, surface and/or volumetric } \\
\text { examinations and leakage tests of all Class } 1,2 \text { and } 3 \\
\text { pressure-retaining components, their supports and } \\
\text { integral attachments, including welds, pump casings, } \\
\text { valve bodies, pressure-retaining bolting, } \\
\text { piping/component supports, and reactor head closure } \\
\text { studs. }\end{array}$ & $\begin{array}{l}\text { Industry: } \\
\text { Numerous instances of degradation of components, component supports, and bolting } \\
\text { have occurred. } \\
\text { Plant Specific: } \\
\text { - Control Rod Drive Housings } \\
\text { - Piping Welds } \\
\text { - Component Supports } \\
\text { - Bolting } \\
\text { - Temperature Element Penetration } \\
\text { - Reactor Coolant Pressurizer Safe End } \\
\text { - Engineered Safeguards Systems Check Valve }\end{array}$ \\
\hline $\begin{array}{l}\text { Bolting } \\
\text { Integrity } \\
\text { Program }\end{array}$ & $\begin{array}{l}\text { This is an existing program that manages the aging } \\
\text { effects associated with bolting through the } \\
\text { performance of periodic inspections. The program } \\
\text { also includes repair/replacement controls for ASME } \\
\text { Section XI related bolting and generic guidance } \\
\text { regarding material selection, thread lubrication and } \\
\text { assembly of bolted joints. }\end{array}$ & $\begin{array}{l}\text { Industry: } \\
\text { Numerous instances of degradation of bolting have occurred. } \\
\text { Plant Specific: } \\
\text { - Piping Flange Bolts (1) } \\
\text { Pump Studs (2) } \\
\text { - Tank Flange Bolts (1) } \\
\text { - Pipe Support Bolting (1) } \\
\text { - ESS Equipment Bolting (1) }\end{array}$ \\
\hline $\begin{array}{l}\text { Boric Acid } \\
\text { Corrosion }\end{array}$ & $\begin{array}{l}\text { This is an existing program that monitors component } \\
\text { degradation due to boric acid leakage through the }\end{array}$ & $\begin{array}{l}\text { Industry: } \\
\text { - Boric acid wastage of reactor coolant system piping and nozzles }\end{array}$ \\
\hline
\end{tabular}




\begin{tabular}{|c|c|c|}
\hline $\begin{array}{c}\text { Aging } \\
\text { Management } \\
\text { Program }\end{array}$ & $\begin{array}{c}\text { Description of Aging Management Program } \\
\text { (AMP) }\end{array}$ & Operating Experience \\
\hline Program & $\begin{array}{l}\text { performance of periodic inspections. It implements } \\
\text { the recommendations of NRC Generic Letter } 88-05 \text {. } \\
\text { The program requires periodic visual inspection of } \\
\text { all systems within the scope of license renewal that } \\
\text { contain borated water for evidence of leakage, } \\
\text { accumulations of dried boric acid, or boric acid } \\
\text { wastage. The program also provides for visual } \\
\text { inspections and early discovery of borated water } \\
\text { leaks such that structures, electrical and mechanical } \\
\text { components that may be contacted by leaking } \\
\text { borated water will not be adversely affected such } \\
\text { that their intended functions are impaired. }\end{array}$ & $\begin{array}{l}\text { - Boric acid corrosion of reactor vessel head and closure studs from leaking borated } \\
\text { water } \\
\text { - Failure of valve packing gland bolts due to boric acid wastage } \\
\text { - Failure of valve body to bonnet studs/nuts due to boric acid wastage } \\
\text { - Boric acid wastage of reactor coolant pump closure flange studs } \\
\text { - Boric acid corrosion of steam generator manway closure studs } \\
\text { - Boric acid corrosion of high pressure safety injection pump casing } \\
\text { Plant Specific: } \\
\text { - Boric acid leaks in the containment spray header in containment at flanges with } \\
\text { carbon steel bolting and a threaded spray nozzle connection } \\
\text { - Boric acid wastage of primary coolant pump studs } \\
\text { - Coric acid wastage of manual valve body-to-bonnet bolts } \\
\text { connections due to external boric acid leakage }\end{array}$ \\
\hline $\begin{array}{l}\text { Buried } \\
\text { Services } \\
\text { Corrosion } \\
\text { Monitoring } \\
\text { Program }\end{array}$ & $\begin{array}{l}\text { This is a new program that manages aging effects on } \\
\text { the external surfaces of carbon steel, low-alloy steel, } \\
\text { and stainless steel components that are buried in soil } \\
\text { or sand. This program includes (a) visual inspections } \\
\text { of external surfaces of buried components for } \\
\text { evidence of coating damage and substrate } \\
\text { degradation to manage the effects of aging, (b) } \\
\text { visual inspection of the external surfaces of buried } \\
\text { stainless steel components for evidence of crevice } \\
\text { corrosion, pitting, and MIC. The periodicity of these } \\
\text { inspections for carbon, low-alloy, and stainless steel } \\
\text { will be based on opportunities for inspection such as } \\
\text { scheduled maintenance work. }\end{array}$ & $\begin{array}{l}\text { Industry: } \\
\text { Issues related to Diesel fuel line leakage from the absence of required coating leading } \\
\text { to corrosion. } \\
\text { Plant Specific: } \\
\text { - Through wall leak in buried steam line. } \\
\text { - Generic program deficiencies from internal Engineering Programs audit. } \\
\text { - See the Fire Protection Program for OE related to buried fire main ruptures. } \\
\text { None of the plant operating issues or instances resulted from normal aging, or reflect } \\
\text { significant program deficiencies. }\end{array}$ \\
\hline $\begin{array}{l}\text { Closed Cycle } \\
\text { Cooling } \\
\text { Water } \\
\text { Program }\end{array}$ & $\begin{array}{l}\text { This is an existing program that manages aging } \\
\text { effects in closed cycle cooling water systems that are } \\
\text { not subject to significant sources of contamination, } \\
\text { in which water chemistry is controlled and heat is } \\
\text { not directly rejected to the ultimate heat sink. The } \\
\text { program includes } \\
\text { (a) maintenance of system }\end{array}$ & $\begin{array}{l}\text { Industry: } \\
\text { - SCC in reactor coolant pump oil cooler discharge piping. } \\
\text { - Corroded solder connections in diesel lube oil cooler due to inadequate corrosion } \\
\text { inhibitor } \\
\text { - Inoperable check valves (stuck open) due to corrosion product buildup } \\
\text { - Cracks in Component Cooling Water piping }\end{array}$ \\
\hline
\end{tabular}




\begin{tabular}{|c|c|c|}
\hline $\begin{array}{l}\text { Aging } \\
\text { Management } \\
\text { Program }\end{array}$ & $\begin{array}{l}\text { Description of Aging Management Program } \\
\text { (AMP) }\end{array}$ & Operating Experience \\
\hline & $\begin{array}{l}\text { corrosion inhibitor concentrations to minimize } \\
\text { degradation, and (b) periodic or one-time testing and } \\
\text { inspections to assess SSC aging. }\end{array}$ & $\begin{array}{l}\text { - Fouling of diesel cooling water heat exchangers } \\
\text { Plant Specific: } \\
\text { - Tube blockage and fouling in Component Cooling Heat Exchanger } \\
\text { - Fuel Pool Heat Exchanger tube breakage due to high Component Cooling Water } \\
\text { flow } \\
\text { - Through wall flaw in Spent Fuel Pool Cooling pipe }\end{array}$ \\
\hline $\begin{array}{l}\text { Containment } \\
\text { Inservice } \\
\text { Inspection } \\
\text { Program }\end{array}$ & $\begin{array}{l}\text { This is an existing program that is designed to ensure } \\
\text { that containment shell concrete, the post-tensioning } \\
\text { system and steel pressure retaining elements } \\
\text { continue to provide an acceptable level of structural } \\
\text { integrity. In addition, it is designed to ensure that the } \\
\text { liner (with associated moisture barriers), other } \\
\text { leakage limiting steel barriers and pressure retaining } \\
\text { bolted connections have not degraded. }\end{array}$ & $\begin{array}{l}\text { Industry: } \\
\text { Instances have occurred with containments. } \\
\text { Plant Specific: } \\
\text { - Liner plate corrosion } \\
\text { - Unacceptable tendon liftoff value } \\
\text { - Tendon gallery corrosion } \\
\text { - Tendon grease leakage } \\
\text { - Moisture barrier not in place } \\
\text { - Tendon sheath water intrusion }\end{array}$ \\
\hline $\begin{array}{l}\text { Diesel Fuel } \\
\text { Monitoring } \\
\text { and Storage } \\
\text { Program }\end{array}$ & $\begin{array}{l}\text { This is an existing program that assures the } \\
\text { continued availability and quality of fuel oil to be } \\
\text { used in diesel generators and diesel fire pumps. The } \\
\text { program includes (a) monitoring and trending of fuel } \\
\text { oil chemistry to maintain fuel oil quality and } \\
\text { mitigate corrosion, (b) periodic draining, cleaning, } \\
\text { and internal inspection of fuel oil storage tanks, and } \\
\text { (c) verification of program effectiveness by a one- } \\
\text { time measurement of fuel oil storage tank bottom } \\
\text { thickness confirming the absence of an aging effect. }\end{array}$ & $\begin{array}{l}\text { Industry: } \\
\text { - Fuel contamination leading to corrosion of fuel oil system components. } \\
\text { - Improper zinc coating curing and epoxy application by the manufacturer leads to } \\
\text { zinc-fuel reaction creating adverse corrosion. } \\
\text { - Fuel oil leak caused by improper outer coating application. } \\
\frac{\text { Plant Specific: }}{\text { No aging issues were identified. }}\end{array}$ \\
\hline $\begin{array}{l}\text { Fire } \\
\text { Protection } \\
\text { Program }\end{array}$ & $\begin{array}{l}\text { This is an existing program that includes (a) fire } \\
\text { barrier inspections, (b) electric and diesel-driven fire } \\
\text { pump tests, and (c) periodic maintenance, testing, } \\
\text { and inspection of water-based fire protection } \\
\text { systems. Periodic visual inspections of fire barrier } \\
\text { penetration seals, fire dampers, fire barrier walls, }\end{array}$ & $\begin{array}{l}\text { Industry: } \\
\text { - Fire water system piping corrosion and ruptures } \\
\text { - Fire retardant coatings and materials } \\
\text { - Fouling of components in contact with raw water } \\
\text { - Problems with fire barriers. }\end{array}$ \\
\hline
\end{tabular}




\begin{tabular}{|c|c|c|}
\hline $\begin{array}{l}\text { Aging } \\
\text { Management } \\
\text { Program }\end{array}$ & $\begin{array}{c}\text { Description of Aging Management Program } \\
\text { (AMP) }\end{array}$ & Operating Experience \\
\hline & $\begin{array}{l}\text { ceilings and floors, and periodic visual inspections } \\
\text { and functional tests of fire-rated doors are performed } \\
\text { to ensure that functionality and operability is } \\
\text { maintained. }\end{array}$ & $\begin{array}{l}\text { Plant Specific: } \\
\text { - Blockage of Fire Protection piping with corrosion products } \\
\text { - Deluge valve trim piping failures due to corrosion } \\
\text { - Underground fire main rupture due to cyclic loadings } \\
\text { - Water tight fire door seal degradation }\end{array}$ \\
\hline $\begin{array}{l}\text { Flow } \\
\text { Accelerated } \\
\text { Corrosion } \\
\text { Program }\end{array}$ & $\begin{array}{l}\text { This is an existing program that manages aging } \\
\text { effects due to flow-accelerated corrosion (FAC) on } \\
\text { the internal surfaces of carbon or low alloy steel } \\
\text { piping, elbows, reducers, expanders, and valve } \\
\text { bodies which contain high energy fluids (both single } \\
\text { phase and two phase). }\end{array}$ & $\begin{array}{l}\text { Industry: } \\
\text { - Feedwater heater shell degradation and ruptures } \\
\text { - Feedwater and Condensate line ruptures } \\
\text { - Pipe wall thinning downstream of control valves and flow restricting devices } \\
\text { - Valve body erosion } \\
\text { - Extraction steam line ruptures } \\
\text { - Moisture Separator Reheater Drain Tank drain line ruptures } \\
\text { - Erosion of carbon steel ribs and tube supports in Steam Generators } \\
\text { Plant Specific: } \\
\text { - FAC on } 2 \text { inch main steam line elbows } \\
\text { - Higher than expected wear rates on } 8 \text { inch steam pipes and elbows on the outlet of } \\
\text { Moisture Separator Reheater } \\
\text { - Main Condenser tube leaks caused by FAC } \\
\text { - Higher than expected wear rates on high pressure extraction steam piping to high } \\
\text { pressure feedwater heater } \\
\text { - FAC on end-bell of low pressure feedwater heater } \\
\text { - Valve body FAC on control valves and check valves } \\
\text { - FAC of feedwater heater shell side capped drains } \\
\text { - FAC damage to low pressure turbine extraction sleeves } \\
\text { - FAC damage to extraction steam lines to high pressure feedwater heaters } \\
\text { - FAC damage to Moisture Separator Reheater vent line } \\
\text { - FAC of feedwater piping } \\
\text { - FAC of reducer downstream of control valve } \\
\text { - Through wall steam leak on steam generator flash tank }\end{array}$ \\
\hline $\begin{array}{l}\text { One-Time } \\
\text { Inspection } \\
\text { Program }\end{array}$ & $\begin{array}{l}\text { This is a new program that addresses potentially } \\
\text { long incubation periods for certain aging effects, } \\
\text { including various corrosion mechanisms, cracking, }\end{array}$ & $\begin{array}{l}\text { Industry: } \\
\text { Not discussed }\end{array}$ \\
\hline
\end{tabular}




\begin{tabular}{|c|c|c|}
\hline $\begin{array}{l}\text { Aging } \\
\text { Management } \\
\text { Program }\end{array}$ & $\begin{array}{c}\text { Description of Aging Management Program } \\
\text { (AMP) }\end{array}$ & Operating Experience \\
\hline & $\begin{array}{l}\text { and selective leaching, and provides a means of } \\
\text { verifying that an aging effect is either not occurring } \\
\text { or progressing so slowly as to have negligible effect } \\
\text { on the intended function of the structure or } \\
\text { component. }\end{array}$ & $\frac{\text { Plant Specific: }}{\text { Not discussed }}$ \\
\hline $\begin{array}{l}\text { Open Cycle } \\
\text { Cooling } \\
\text { Water } \\
\text { Program }\end{array}$ & $\begin{array}{l}\text { This is an existing program that manages aging } \\
\text { effects such as loss of material due to general, } \\
\text { pitting, and crevice corrosion, erosion, MIC, and loss } \\
\text { of heat transfer due to biological/corrosion product } \\
\text { fouling (e.g., sedimentation, silting) caused by } \\
\text { exposure of internal surfaces of metallic components } \\
\text { to raw, untreated (e.g., service) water. The program } \\
\text { scope includes activities to manage aging in the } \\
\text { Service Water System (SWS) and Circulating Water } \\
\text { system (CWS). }\end{array}$ & $\begin{array}{l}\text { Industry: } \\
\text { - Accumulations of silt and corrosion products in service water piping, valves, and } \\
\text { heat exchangers } \\
\text { - Accumulation of biological growth (mussels, clams, and shells) in service water } \\
\text { piping, valves and heat exchangers } \\
\text { - MIC causing pitting attack of carbon steel and stainless steel service water piping, } \\
\text { pump casings, and } 90 / 10 \mathrm{Cu} / \mathrm{Ni} \text { heat exchanger tubes } \\
\text { Plant Specific: } \\
\text { - Defective tubes in the Main Condenser that required plugging due to MIC } \\
\text { - Control Room Condensing Unit Condenser Drain Plug severely corroded due to } \\
\text { - Large Zebra Mussel accumulation near traveling screens and inside intake piping } \\
\text { - Blockage of heat exchanger and cooler tubing } \\
\text { - Corroded service water piping at threaded connections } \\
\text { - Pinhole leaks in service water piping due to MIC } \\
\text { - Switch failure due to sediment and corrosion (galvanic) blocking sensing line } \\
\text { - Tubercles growing in carbon steel service water piping } \\
\text { - Erosion of pipes, cooling coils, and heat exchanger tubes causing service water leaks }\end{array}$ \\
\hline $\begin{array}{l}\text { Reactor } \\
\text { Vessel } \\
\text { Integrity } \\
\text { Surveillance } \\
\text { Program }\end{array}$ & $\begin{array}{l}\text { This is an existing program that manages the aging } \\
\text { effect reduction of fracture toughness due to neutron } \\
\text { embrittlement of the low alloy steel reactor vessel. }\end{array}$ & $\begin{array}{l}\text { Industry \& Plant Specific: } \\
\text { GL 92-01, Revision 1, "Reactor Vessel Structural Integrity," and Supplement } 1 \text { to GL } \\
\text { 92-01, Revision 1, "Reactor Vessel Structural Integrity." Palisades' response to these } \\
\text { documents has been incorporated into the Reactor Vessel Integrity Surveillance } \\
\text { Program. } \\
\text { A review of NRC Inspection Reports, QA Audit/Surveillance Reports, and Self } \\
\text { Assessments since } 1999 \text { revealed no issues or findings that could impact the } \\
\text { effectiveness of the Reactor Vessel Surveillance Program. }\end{array}$ \\
\hline Reactor & The Reactor Vessel Internals Inspection Program is & Industry: \\
\hline
\end{tabular}




\begin{tabular}{|c|c|c|}
\hline $\begin{array}{c}\text { Aging } \\
\text { Management } \\
\text { Program }\end{array}$ & $\begin{array}{c}\text { Description of Aging Management Program } \\
\text { (AMP) }\end{array}$ & Operating Experience \\
\hline $\begin{array}{l}\text { Vessel } \\
\text { Internals } \\
\text { Inspection } \\
\text { Program }\end{array}$ & $\begin{array}{l}\text { an existing program that manages the aging effects } \\
\text { for reactor vessel internals. }\end{array}$ & $\begin{array}{l}\text { Several instances were revealed where degradation has occurred within the reactor } \\
\text { vessel internals. } \\
\text { Plant Specific: } \\
\text { Degradation was discovered in the core barrel and the control rod drive mechanism } \\
\text { (CRDM) seal housings }\end{array}$ \\
\hline $\begin{array}{l}\text { Steam } \\
\text { Generator } \\
\text { Tube } \\
\text { Integrity } \\
\text { Program }\end{array}$ & $\begin{array}{l}\text { This is an existing program that manages the aging } \\
\text { effects of steam generator tubes and tube repairs. } \\
\text { The Program also manages the aging effects of } \\
\text { accessible steam generator secondary side internal } \\
\text { components and incorporates the guidance of NEI } \\
97-06 \text {. }\end{array}$ & $\begin{array}{l}\text { Industry: } \\
\text { Instances of degradation have occurred within the steam generators. } \\
\text { Plant Specific: } \\
\text { - Top of tubesheet } \\
\text { - Within the tubesheet } \\
\text { - U-bends } \\
\text { - Mechanical wear at eggcrate supports, vertical straps, and diagonal bars }\end{array}$ \\
\hline $\begin{array}{l}\text { Structural } \\
\text { Monitoring } \\
\text { Program }\end{array}$ & $\begin{array}{l}\text { This is an existing program that is designed to ensure } \\
\text { that age related (as well as other) deterioration of } \\
\text { plant structures (including masonry walls) and } \\
\text { components within its scope is appropriately } \\
\text { managed to ensure that each such structure or } \\
\text { component retains the ability to perform its intended } \\
\text { function. }\end{array}$ & $\begin{array}{l}\text { Industry: } \\
\text { - Corrosion of steel ice condenser containment vessels caused by boric acid and } \\
\text { condensation } \\
\text { - Cracks in concrete floors caused by flexing and shrinkage } \\
\text { Plant Specific: } \\
\text { - Settling of air compressor foundations } \\
\text { - Watertight barrier degradation } \\
\text { - Spalled concrete and exposed anchor bolts } \\
\text { - Cracking of concrete beams in the Auxiliary Building } \\
\text { - Corrosion of condenser rock anchors caused by standing water and debris } \\
\text { - Degradation of snubber anchor support structure concrete and grout } \\
\text { - Deterioration of floor plugs due to leaking water } \\
\text { - Moisture Separator Reheater foundation cracking } \\
\text { - Cracks in concrete duct bank } \\
\text { - Cracks in West ESS room west wall } \\
\text { - Spalled concrete on wall of 1-2 Diesel Generator Exhaust Plenum } \\
\text { - Groundwater leaks in Auxiliary Feedwater Pump room floor } \\
\end{array}$ \\
\hline System & This is an existing plant-specific program that & Industry: \\
\hline
\end{tabular}




\begin{tabular}{|c|c|c|}
\hline $\begin{array}{c}\text { Aging } \\
\text { Management } \\
\text { Program }\end{array}$ & $\begin{array}{l}\text { Description of Aging Management Program } \\
\text { (AMP) }\end{array}$ & Operating Experience \\
\hline $\begin{array}{l}\text { Monitoring } \\
\text { Program }\end{array}$ & $\begin{array}{l}\text { manages aging effects for normally accessible, } \\
\text { external surfaces of piping, tanks, and other } \\
\text { components and equipment within the scope of } \\
\text { License Renewal. These aging effects are managed } \\
\text { through visual inspection and monitoring of external } \\
\text { surfaces for leakage and evidence of material } \\
\text { degradation. }\end{array}$ & $\begin{array}{l}\text { - Service Water Pump flange welds and bolting found excess rusting leading } \\
\text { to leakage (Inadequate/infrequent system walkdowns were cited). } \\
\text { Plant Specific: } \\
\text { - Various pump and valve flange welds and bolting (carbon steel) were found having } \\
\text { significant material loss due to high moisture environment or boric acid } \\
\text { accumulations. } \\
\text { - Floor-mounted pipe supports were discovered with excessive corrosion of bolts. } \\
\text { (Concrete failure may have contributed from vibration and/or concrete boric acid } \\
\text { contamination) }\end{array}$ \\
\hline $\begin{array}{l}\text { Water } \\
\text { Chemistry } \\
\text { Program }\end{array}$ & $\begin{array}{l}\text { This is an existing program that is credited for } \\
\text { managing aging effects such as loss-of-material due } \\
\text { to general, pitting and crevice corrosion; cracking } \\
\text { due to SCC; and steam generator tube degradation } \\
\text { caused by denting, intergranular attack (IGA) and } \\
\text { outer diameter stress corrosion cracking (ODSCC), } \\
\text { by controlling the environment to which internal } \\
\text { surfaces of systems and components are exposed. } \\
\text { The aging effects are minimized by controlling the } \\
\text { chemical species that cause the underlying } \\
\text { mechanisms that result in these aging effects. }\end{array}$ & $\begin{array}{l}\text { Industry: } \\
\text { - Cracking in steam generator welds } \\
\text { - Cracking and pitting of steam generator tubes and components } \\
\text { - Alloy } 600 \text { cracking } \\
\text { - Thinning of pipe and components due to erosion/corrosion } \\
\text { - Cracking in safety injection accumulator nozzles } \\
\text { - High wear of Reactor Coolant Pump Aluminum Oxide coated seals } \\
\text { - Cracking of Control Rod Drive Housings } \\
\text { - Cracking of pressurizer instrument tap nozzles } \\
\text { - Cracking of safety injection piping } \\
\text { - Cracking in feedwater piping } \\
\text { - Chemical impurity intrusions into primary and secondary systems } \\
\text { - Resin intrusions into the primary coolant systems } \\
\text { Plant Specific: } \\
\text { - Defective tubes in the Main Condenser due to steam impingement wear and } \\
\text { - Excrobiologically Influenced Corrosion (MIC) pitting } \\
\end{array}$ \\
\hline
\end{tabular}


Table 4-5 License Renewal Applications for Information Related to Aging Degradation - Point Beach Nuclear Power Plant Units 1 \& 2 [Available on the NRC Website]

\begin{tabular}{|c|c|c|}
\hline $\begin{array}{c}\text { Aging } \\
\text { Management } \\
\text { Program }\end{array}$ & $\begin{array}{c}\text { Description of Aging Management Program } \\
\text { (AMP) }\end{array}$ & Operating Experience \\
\hline $\begin{array}{l}\text { ASME Section } \\
\text { XI, } \\
\text { Subsections } \\
\text { IWE \& IWL } \\
\text { Inservice } \\
\text { Inspection } \\
\text { Program }\end{array}$ & $\begin{array}{l}\text { This AMP manages aging of (a) steel liners of } \\
\text { concrete containments and their integral } \\
\text { attachments; containment hatches and airlocks; } \\
\text { seals, gaskets and moisture barriers; and pressure } \\
\text { retaining bolting, and (b) reinforced concrete } \\
\text { containments and unbonded post-tensioning } \\
\text { systems. }\end{array}$ & $\begin{array}{l}\text { Industry: } \\
\text { Industry operating experience and NRC information notices have documented areas of } \\
\text { concern regarding containment liner plate, concrete, and tendon degradation. } \\
\text { Plant Specific: } \\
\text { Plant specific operating experience has shown that degradation has occurred. For } \\
\text { example: failed tendon wires, missing or broken components found in the tendon } \\
\text { hardware, degraded concrete in containment structure, corroded containment liner, } \\
\text { and corrosion of penetrations inside of containment. } \\
\text { - Degradation has occurred in the Unit } 1 \text { and } 2 \text { containment liners at the } 8 \text { foot } \\
\text { elevation due to poor condition of the moisture barriers. The degradation consisted } \\
\text { of general corrosion and pitting. } \\
\text { - Several mechanical penetrations inside the Unit } 1 \text { and } 2 \text { containments have shown } \\
\text { indications of general corrosion and/or peeling paint. } \\
\text { - Corrosion was also found in the Unit } 1 \text { and } 2 \text { Containment Sump A at the interface } \\
\text { between the containment liner plates and containment floor slabs. } \\
\text { - Inspections performed on the containment tendons have discovered the following } \\
\text { degradations: } \\
\text { Broken wires, } \\
\text { Wires with } 2 \% \text { to } 4 \% \text { less than expected pre-stress, } \\
\text { Presence of nitrates in grease, } \\
\text { Cracked button-head, missing button-heads, } \\
\text { Tendon void of } 9.7 \% \text { of grease volume, } \\
\text { More grease added than removed in some instances. }\end{array}$ \\
\hline $\begin{array}{l}\text { ASME Section } \\
\text { XI, Subsection } \\
\text { IWF Inservice } \\
\text { Inspection } \\
\text { Program }\end{array}$ & $\begin{array}{l}\text { This AMP manages aging effects for Class } 1,2 \text {, and } \\
3 \text { component supports. }\end{array}$ & $\begin{array}{l}\text { Industry: } \\
\text { NRC Information Notice } 80-36 \text { notified utilities of the potential for stress corrosion } \\
\text { cracking (SCC) of high strength component support bolts. } \\
\text { Plant Specific: } \\
\text { The most common relevant condition discovered by this AMP has been loose }\end{array}$ \\
\hline
\end{tabular}




\begin{tabular}{|c|c|c|}
\hline $\begin{array}{l}\text { Aging } \\
\text { Management } \\
\text { Program }\end{array}$ & $\begin{array}{c}\text { Description of Aging Management Program } \\
\text { (AMP) }\end{array}$ & Operating Experience \\
\hline & & $\begin{array}{l}\text { fasteners in supports. Loose fasteners are a maintenance issue, rather than a sign of } \\
\text { age-related degradation. }\end{array}$ \\
\hline $\begin{array}{l}\text { Bolting } \\
\text { Integrity } \\
\text { Program }\end{array}$ & $\begin{array}{l}\text { This AMP manages the aging effects associated } \\
\text { with bolting through the performance of periodic } \\
\text { inspections. The program also includes } \\
\text { repair/replacement controls for ASME Section XI } \\
\text { related bolting and generic guidance regarding } \\
\text { material selection, thread lubrication and assembly } \\
\text { of bolted joints. }\end{array}$ & $\begin{array}{l}\text { Industry: } \\
\text { Numerous instances of primary pressure boundary degradation. There have been } \\
\text { various NRC communications including information notices, bulletins, and generic } \\
\text { letters on bolting degradation. Most instances of degradation fall into two categories: } \\
\text { boric acid corrosion caused by leakage at mechanical joints; and degradation of high } \\
\text { strength bolting caused by stress corrosion cracking. General corrosion of bolting and } \\
\text { fasteners has also occurred for structural bolting located in a humid environment. } \\
\text { Plant Specific: } \\
\text { Boric acid wastage on one body/bonnet check valve stud. General corrosion was also } \\
\text { found on structural steel bolting. There were also a few instances of improper bolting } \\
\text { material and torque values being used. There were no incidents of loss of intended } \\
\text { function of a component or system due to fastener degradation. }\end{array}$ \\
\hline $\begin{array}{l}\text { Boraflex } \\
\text { Monitoring } \\
\text { Program }\end{array}$ & $\begin{array}{l}\text { The Boraflex Monitoring Program manages aging } \\
\text { effects for the Boraflex material in the spent fuel } \\
\text { racks. }\end{array}$ & $\begin{array}{l}\text { Industry: } \\
\text { NRC Information Notice IN } 87-43 \text { addresses the problems of development of tears } \\
\text { and gaps in Boraflex sheets due to gamma radiation-induced shrinkage of the } \\
\text { material. NRC IN 93-70, NRC IN } 95-38 \text { and NRC GL } 96-04 \text { address several cases of } \\
\text { significant degradation of Boraflex test coupons due to accelerated dissolution of } \\
\text { Boraflex caused by spent fuel pool water flow through panel enclosures and high } \\
\text { accumulated gamma dose. } \\
\text { Plant Specific: } \\
\text { The latest inspection of the SFP Boraflex panels was conducted in August } 2001 \text {. The } \\
\text { results of the Blackness Test (neutron attenuation measurements) indicated that for the } \\
\text { first time since the Boraflex panels have been inspected, gaps have been found in } 27 \\
\text { panels ranging from } 0.8 \text { inches to } 3.4 \text { inches. }\end{array}$ \\
\hline $\begin{array}{l}\text { Boric Acid } \\
\text { Corrosion } \\
\text { Program }\end{array}$ & $\begin{array}{l}\text { This AMP manages aging effects for structures and } \\
\text { components as a result of borated water leakage. } \\
\text { The program requires periodic visual inspection of } \\
\text { systems that contain borated water for evidence of } \\
\text { leakage or accumulations of dried boric acid. }\end{array}$ & $\begin{array}{l}\text { Industry: } \\
\text { Boric acid solution leaking from the Reactor Coolant System can cause significant } \\
\text { corrosion damage to carbon steel reactor coolant pressure boundary components. } \\
\text { Severe corrosion damage to the RPV head at Davis-Besse and observed cracking and } \\
\text { leakage on the RPV bottom head penetrations at South Texas have resulted in much } \\
\text { industry attention to ensuring the implementation of an effective boric acid corrosion }\end{array}$ \\
\hline
\end{tabular}




\begin{tabular}{|c|c|c|}
\hline $\begin{array}{c}\text { Aging } \\
\text { Management } \\
\text { Program }\end{array}$ & $\begin{array}{c}\text { Description of Aging Management Program } \\
\text { (AMP) }\end{array}$ & Operating Experience \\
\hline & & $\begin{array}{l}\text { program. } \\
\text { Plant Specific: } \\
\text { Numerous Work Orders, Condition Reports/Action Requests, and several Licensee } \\
\text { Event Reports have been issued as a result of this AMP discovering boric acid leaks } \\
\text { and corrosion of components due to borated water leakage. A large percentage of } \\
\text { these described finding dried boric acid crystal deposits either on the component from } \\
\text { which it leaked or on the floor below the leaking component. Occasionally, dried } \\
\text { boric acid crystals were found on components located below the leaking component. }\end{array}$ \\
\hline $\begin{array}{l}\text { Buried } \\
\text { Services } \\
\text { Monitoring } \\
\text { Program }\end{array}$ & $\begin{array}{l}\text { This AMP manages aging effects on the external } \\
\text { surfaces of carbon steel, low-alloy steel, and cast } \\
\text { iron components (e.g., tanks, piping) that are buried } \\
\text { in soil or sand. }\end{array}$ & $\begin{array}{l}\text { Industry: } \\
\text { Carbon steel, low alloy steel, or cast iron buried components have experienced } \\
\text { corrosion and selective leaching degradation. The critical areas appear to be at the } \\
\text { interface where the component transitions from above ground to below ground. This } \\
\text { is also the area where coatings and wrappings will most likely be missing or damaged. } \\
\text { Plant Specific: } \\
\text { No degraded conditions discussed. }\end{array}$ \\
\hline $\begin{array}{l}\text { Closed-Cycle } \\
\text { Cooling Water } \\
\text { System } \\
\text { Surveillance } \\
\text { Program }\end{array}$ & $\begin{array}{l}\text { This AMP manages aging effects in closed cycle } \\
\text { cooling water systems that are not subject to } \\
\text { significant sources of contamination, in which } \\
\text { water chemistry is controlled and heat is not } \\
\text { directly rejected to the ultimate heat sink. }\end{array}$ & $\begin{array}{l}\text { Industry: } \\
\text { Not discussed } \\
\text { Plant Specific: } \\
\text { The closed-cycle (CC) System performance has been very good. PBNP has not } \\
\text { experienced degradation of its CC System due to corrosion product build up or } \\
\text { cracking. } \\
\text { Tube vibration in the CC Heat Exchangers has been documented. The vibration has } \\
\text { been attributed to increased clearances in the tube to tube support plate interface. The } \\
\text { CC Heat Exchangers were re-tubed with SeaCure tube material, which creates the } \\
\text { potential for galvanic corrosion of the carbon steel tube support plates. Galvanic } \\
\text { corrosion of the tube support plates is believed to be the reason for the increased } \\
\text { clearances and subsequent tube vibration at high CC flows. } \\
\text { Trending of nitrite and microbiological levels in the engine coolant of G01 and G02 } \\
\text { EDGs has revealed slight in-leakage of service water into the engine coolant. }\end{array}$ \\
\hline Fire Protection & This AMP includes (a) fire barrier inspections, (b) & Industry: \\
\hline
\end{tabular}




\begin{tabular}{|c|c|c|}
\hline $\begin{array}{c}\text { Aging } \\
\text { Management } \\
\text { Program }\end{array}$ & $\begin{array}{c}\text { Description of Aging Management Program } \\
\text { (AMP) }\end{array}$ & Operating Experience \\
\hline Program & $\begin{array}{l}\text { electric and diesel-driven fire pump tests, (c) } \\
\text { periodic inspection and testing of the halon fire } \\
\text { suppression system, and (d) periodic maintenance, } \\
\text { testing, and inspection of water-based fire } \\
\text { protection systems. }\end{array}$ & $\begin{array}{l}\text { Not discussed } \\
\text { Plant Specific: } \\
\text { Some cases of small pipe threaded connection leaks, small pipe external corrosion } \\
\text { leaks, spray nozzles for the transformer deluge system plugged due to rust scale build- } \\
\text { up, and cracked piping and fittings. Pinhole leaks have also been found on the } 10 \text { inch } \\
\text { fire water supply header and sprinkler heads have been found to leak. Inspections } \\
\text { performed on the fire hydrants did reveal that three hydrants were found to be stuck } \\
\text { shut over a ten year inspection period. None have been found stuck during the last few } \\
\text { inspections. Plant-specific operating experience has indicated that below grade fire } \\
\text { system piping leaks are very rare. The sandy soil condition is such that it is not } \\
\text { conducive to high rates of corrosion and the Lake Michigan water used for the fire } \\
\text { protection system is not aggressive to the internal surfaces of the piping. Fire doors } \\
\text { are occasionally found in need of repair. Some of the electrical penetration fire seals } \\
\text { in containment were also found in need of repair. }\end{array}$ \\
\hline $\begin{array}{l}\text { Flow- } \\
\text { Accelerated } \\
\text { Corrosion } \\
\text { Program }\end{array}$ & $\begin{array}{l}\text { This AMP manages aging effects due to flow- } \\
\text { accelerated corrosion (FAC) on the internal } \\
\text { surfaces of carbon or low alloy steel piping, } \\
\text { elbows, reducers, expanders, and valve bodies } \\
\text { which contain high energy fluids (both single phase } \\
\text { and two phase). The program implements the EPRI } \\
\text { guidelines in NSAC-202L-R2 for an effective FAC } \\
\text { program and includes (a) an analysis using a } \\
\text { predictive code such as CHECWORKS to } \\
\text { determine critical locations, (b) baseline inspections } \\
\text { to determine the extent of thinning at these } \\
\text { locations, (c) follow-up inspections to confirm the } \\
\text { predictions, and (d) repairing or replacing } \\
\text { components, as necessary. The FAC Program has } \\
\text { been an ongoing program at PBNP since } 1987 \text { in } \\
\text { response to NRC IEB } 87-01 \text {. }\end{array}$ & $\begin{array}{l}\text { Industry: } \\
\text { There are many instances where components have failed in service due to component } \\
\text { wall thinning and rupturing due to erosion. A large number of these failures occurred } \\
\text { in two phase systems (saturated steam) where a change in geometry exists. Operating } \\
\text { experience has also shown failures occurring in single phase systems mostly where a } \\
\text { change in geometry exists. } \\
\text { Plant Specific: } \\
\text { In } 1999 \text {, Unit } 1 \text { experienced a plant shutdown due to steam leaking from } \\
\text { feedwater heater 4B. Wall thinning due to steam impingement and FAC had occurred } \\
\text { in the heater shell causing the leak. Inspection of similar Unit } 1 \text { feedwater heaters } \\
\text { indicated that they required repairs due to wall thinning. Inspection of the Unit } 2 \\
\text { feedwater heaters revealed no comparable wall thinning. Root cause analysis of the } \\
\text { event also discovered that the materials of construction for the Unit } 1 \text { feedwater } \\
\text { heaters did not contain a sufficient amount of chromium or molybdenum to help } \\
\text { mitigate FAC effects. The original design did not specify for chromium or } \\
\text { molybdenum content. Unit } 2 \text { feedwater heater materials contain an adequate amount } \\
\text { of chromium and molybdenum to mitigate the effects of FAC. }\end{array}$ \\
\hline
\end{tabular}




\begin{tabular}{|c|c|c|}
\hline $\begin{array}{c}\text { Aging } \\
\text { Management } \\
\text { Program }\end{array}$ & $\begin{array}{l}\text { Description of Aging Management Program } \\
\text { (AMP) }\end{array}$ & Operating Experience \\
\hline $\begin{array}{l}\text { Fuel Oil } \\
\text { Chemistry } \\
\text { Control } \\
\text { Program }\end{array}$ & $\begin{array}{l}\text { This AMP mitigates and manages aging effects on } \\
\text { the internal surfaces of fuel oil storage tanks and } \\
\text { associated components in systems that contain fuel } \\
\text { oil. The program includes (a) surveillance and } \\
\text { monitoring procedures for maintaining fuel oil } \\
\text { quality by controlling contaminants in accordance } \\
\text { with applicable ASTM Standards, (b) periodic } \\
\text { draining of water from fuel oil tanks, (c) periodic or } \\
\text { conditional visual inspection of internal surfaces or } \\
\text { wall thickness measurements (e.g., by UT) from } \\
\text { external surfaces of fuel oil tanks, and (d) one-time } \\
\text { inspections of a representative sample of } \\
\text { components in systems that contain fuel oil. }\end{array}$ & $\begin{array}{l}\text { Industry: } \\
\text { The operating experience of some plants has included identification of water in the } \\
\text { fuel, particulate contamination, and biological fouling. } \\
\text { Plant Specific: } \\
\text { The internals of the above ground fuel oil tanks and the underground emergency fuel } \\
\text { tank were inspected in August of } 2000 \text { and no significant rust deposits, corrosion, or } \\
\text { other obvious defects were found. Thickness measurements of the underground } \\
\text { emergency fuel tank and the bottom of the above ground fuel oil tanks were } \\
\text { performed and indicated no significant loss of material. }\end{array}$ \\
\hline $\begin{array}{l}\text { One-Time } \\
\text { Inspection } \\
\text { Program }\end{array}$ & $\begin{array}{l}\text { This AMP addresses potentially long incubation } \\
\text { periods for certain aging effects and provides a } \\
\text { means of verifying that an aging effect is either not } \\
\text { occurring or progressing so slowly as to have } \\
\text { negligible effect on the intended function of the } \\
\text { structure or component. }\end{array}$ & $\begin{array}{l}\text { Industry: } \\
\text { NA } \\
\text { Plant Specific: } \\
\text { Not applicable because this is a new program to be implemented before } \\
\text { the current operating license expires. }\end{array}$ \\
\hline $\begin{array}{l}\text { Open-Cycle } \\
\text { Cooling } \\
\text { (Service) } \\
\text { Water System } \\
\text { Surveillance } \\
\text { Program }\end{array}$ & $\begin{array}{l}\text { This AMP manages aging effects caused by } \\
\text { exposure of internal surfaces of metallic } \\
\text { components in water systems (e.g., piping, valves, } \\
\text { heat exchangers) to raw, untreated (e.g., service) } \\
\text { water. }\end{array}$ & $\begin{array}{l}\text { Industry: } \\
\text { Heat exchangers have experienced erosion/corrosion of end bells, biofouling build-up, } \\
\text { and silt accumulation. Erosion/corrosion has also been experienced at or near throttled } \\
\text { valves. Zebra mussels have been found. Piping systems have experienced corrosion, } \\
\text { pitting, MIC, and sedimentation build-up especially in low flow areas and stagnant } \\
\text { dead legs off the main flow stream. } \\
\text { Plant Specific: } \\
\text { Descriptions of some of the typical deficiencies found in Service Water System } \\
\text { components are: } \\
\text { - Component Cooling Water heat exchangers experienced corrosion in the wall and } \\
\text { nozzle area of the outlet channel head. } \\
\text { - Localized pitting was found in the Service Water piping supply to the old Spent Fuel } \\
\text { Pool heat exchanger. UT examination revealed that } 68 \% \text { wall thinning had occurred }\end{array}$ \\
\hline
\end{tabular}




\begin{tabular}{|c|c|c|}
\hline $\begin{array}{l}\text { Aging } \\
\text { Management } \\
\text { Program }\end{array}$ & $\begin{array}{c}\text { Description of Aging Management Program } \\
\text { (AMP) }\end{array}$ & Operating Experience \\
\hline & & $\begin{array}{l}\text { and silt was found in the pipe. } \\
\text { - Leakage was found in the Component Cooling Water heat exchanger blowdown } \\
\text { lines. } \\
\text { - Deep pitting due to MIC was found on the G01 Diesel Generator heat exchangers. } \\
\text { The pitting occurred beneath deposits formed by iron oxidizing bacteria. } \\
\text { - Radiography of the K-3A Service Water Air Compressor After Cooler heat } \\
\text { exchanger showed significant wall thinning due to internal corrosion. The heat } \\
\text { exchanger also exhibited blockage due to nodule buildup. } \\
\text { - The G01 Diesel Generator heat exchangers (HX-55A-2 and HX -55A-1) were found } \\
\text { to have significant erosion/corrosion at the south end bells. } \\
\text { - Spent Fuel Pool heat exchanger HX-13A outlet valve body was found to be severely } \\
\text { pitted and eroded. }\end{array}$ \\
\hline $\begin{array}{l}\text { Periodic } \\
\text { Surveillance } \\
\text { and Preventive } \\
\text { Maintenance } \\
\text { Program }\end{array}$ & $\begin{array}{l}\text { This AMP is an existing plant-specific program that } \\
\text { manages aging effects for certain SSCs within the } \\
\text { scope of license renewal. The program provides for } \\
\text { inspection, examination, or testing of selected } \\
\text { structures and components, including fasteners, for } \\
\text { evidence of age-related degradation on a specified } \\
\text { frequency based on operating experience or other } \\
\text { requirements (e.g., Technical Specification or code } \\
\text { requirements). Additionally, the program provides } \\
\text { for replacement of certain components on a } \\
\text { specified frequency based on operating experience. } \\
\text { This AMP is also used to verify the effectiveness of } \\
\text { other aging management programs. }\end{array}$ & $\begin{array}{l}\text { Industry: } \\
\text { NA } \\
\text { Plant Specific: } \\
\text { Not discussed }\end{array}$ \\
\hline $\begin{array}{l}\text { Reactor } \\
\text { Coolant } \\
\text { System Alloy } \\
600 \text { Inspection } \\
\text { Program }\end{array}$ & $\begin{array}{l}\text { This AMP manages crack initiation and growth due } \\
\text { to primary water stress corrosion cracking } \\
\text { (PWSCC) of RCS pressure boundary nickel-based } \\
\text { alloy components (e.g., Alloy } 600 / 690 \text { reactor } \\
\text { vessel/head penetration nozzles, Inconel } 82 / 182 \text {, } \\
82 / 152 \text {, and 52/152 weld joints). }\end{array}$ & $\begin{array}{l}\text { Industry: } \\
\text { Numerous occurrences of cracks and leaks of Alloy } 600 \text { nozzles and penetrations with } \\
\text { partial penetration welds. Most of the cracks of Alloy } 600 \text { penetrations caused by } \\
\text { PWSCC have initiated on the inner diameter of the penetration near the elevation of } \\
\text { the J-groove weld and have been short and axially oriented. Recently, a few PWRs } \\
\text { have experienced circumferential cracking, outer diameter initiated cracking, and } \\
\text { cracking initiating in the J-groove weld, in CRDM penetrations. A PWR has } \\
\text { experienced a throughwall PWSCC crack in an Inconel } 82 / 182 \text { piping butt weld, one } \\
\text { PWR has experienced severe degradation of the RPV head due to boric acid corrosion }\end{array}$ \\
\hline
\end{tabular}




\begin{tabular}{|c|c|c|}
\hline $\begin{array}{c}\text { Aging } \\
\text { Management } \\
\text { Program }\end{array}$ & $\begin{array}{c}\text { Description of Aging Management Program } \\
\text { (AMP) }\end{array}$ & Operating Experience \\
\hline & & $\begin{array}{l}\text { resulting from a VHP leak and another PWR has detected axial cracking in reactor } \\
\text { vessel BMI penetrations. } \\
\text { Plant Specific: } \\
\text { No plant specific aging related cases identified. }\end{array}$ \\
\hline $\begin{array}{l}\text { Reactor Vessel } \\
\text { Internals } \\
\text { Program }\end{array}$ & $\begin{array}{l}\text { This AMP manages the aging effects for reactor } \\
\text { vessel internals (RVI). }\end{array}$ & $\begin{array}{l}\text { Industry: } \\
\text { Most of the industry operating experience reviewed has involved cracking of } \\
\text { austenitic stainless steel baffle-former bolts, or SCC of high-strength internals bolting. } \\
\text { SCC of guide tube split pins has also been reported. } \\
\text { Plant Specific: } \\
\text { An augmented examination via UT was conducted on the baffle-former bolts of } \\
\text { PBNP-2. The UT examination identified a number of bolts with indications indicative } \\
\text { of crack like flaws. }\end{array}$ \\
\hline $\begin{array}{l}\text { Reactor Vessel } \\
\text { Surveillance } \\
\text { Program }\end{array}$ & $\begin{array}{l}\text { The Reactor Vessel Surveillance Program manages } \\
\text { the aging effect reduction of fracture toughness due } \\
\text { to neutron embrittlement of the low alloy steel } \\
\text { reactor vessels. }\end{array}$ & $\begin{array}{l}\text { Industry: } \\
\text { Industry operating experience related to this AMP includes GL 92-01, Revision 1, } \\
\text { "Reactor Vessel Structural Integrity," and Supplement } 1 \text { to GL 92-01, Revision 1, } \\
\text { "Reactor Vessel Structural Integrity." } \\
\text { Plant Specific: } \\
\text { PBNP-1 and PBNP-2 have generally operated successfully within their licensed } \\
\text { Pressure-Temperature (P-T) limits. The current P-T curves for PBNP-1 and PBNP-2 } \\
\text { are valid until } 34.0 \text { Effective Full Power Years (EFPY). New PT curves are developed } \\
\text { and issued, as required. }\end{array}$ \\
\hline $\begin{array}{l}\text { Steam } \\
\text { Generator } \\
\text { Integrity } \\
\text { Program }\end{array}$ & $\begin{array}{l}\text { This AMP incorporates the guidance of NEI } 97-06 \\
\text { and maintains the integrity of the steam generators } \\
\text { (SG), including tubes, tube plugs or other tube } \\
\text { repairs, and various secondary-side internal } \\
\text { components. }\end{array}$ & $\begin{array}{l}\text { Industry: } \\
\text { Supplement } 1 \text { of NRC Information Notice (IN) } 2002-21 \text { discusses Outside Diameter } \\
\text { SCC (ODSCC) found in Alloy } 600 \text { SG tubes at Seabrook. The ODSCC at Seabrook } \\
\text { was caused by high residual stresses resulting from non-optimal tube processing and } \\
\text { could generically affect mill-annealed Alloy } 600 \text {, thermally treated Alloy } 600 \text {, or } \\
\text { thermally treated Alloy } 690 \text { SG tubes. } \\
\text { Plant Specific: } \\
\text { The most recent Unit } 1 \text { and } 2 \text { SG inspection results indicate that they are in very good } \\
\text { condition. For Unit } 1 \text { only } 10 \text { of the } 6,428 \text { heat transfer tubes have been plugged. For }\end{array}$ \\
\hline
\end{tabular}




\begin{tabular}{|c|c|c|}
\hline $\begin{array}{c}\text { Aging } \\
\text { Management } \\
\text { Program }\end{array}$ & $\begin{array}{c}\text { Description of Aging Management Program } \\
\text { (AMP) }\end{array}$ & Operating Experience \\
\hline & & $\begin{array}{l}\text { Unit } 2 \text { only } 4 \text { of the } 6,998 \text { heat transfer tubes have } \\
\text { been plugged. No tubes have been plugged because of corrosion type degradation, } \\
\text { which is consistent with the experience of all other SGs with thermally treated Alloy } \\
600 \text { heat transfer tubes. Secondary-side inspections of the current SGs to date have } \\
\text { revealed no degradation of the swirl vane, moisture separator, feed ring areas, J- } \\
\text { Tubes, or tube support plates. There has been no evidence of wrapper drop on any of } \\
\text { the SGs. }\end{array}$ \\
\hline $\begin{array}{l}\text { Structures } \\
\text { Monitoring } \\
\text { Program }\end{array}$ & $\begin{array}{l}\text { This AMP manages the aging effects associated } \\
\text { with steel (including fasteners), concrete (including } \\
\text { masonry block and grout), earthen berms, and } \\
\text { elastomers. The environments include below grade } \\
\text { and fluid exposed material, outdoor weather, and } \\
\text { indoor air. The program includes all safety related } \\
\text { buildings, structures within the containment, other } \\
\text { buildings within the scope of license renewal, crane } \\
\text { bridge and trolley structures, and component } \\
\text { supports (including HELB structures, panels, etc.) } \\
\text { within the scope of license renewal. }\end{array}$ & $\begin{array}{l}\text { Industry: } \\
\text { Industry operating experience has shown that degradation occurs in structural steel } \\
\text { and concrete components. } \\
\text { Plant Specific: } \\
\text { The inspections performed at PBNP as part of the Structures Monitoring Program } \\
\text { have revealed that degradation has occurred in both concrete and structural steel } \\
\text { components. } \\
\text { Cracks in masonry walls have been found primarily at the mortar joints and these } \\
\text { findings have been documented and resolved. } \\
\text { Concrete structure inspections have been and continue to be a large part of this AMP } \\
\text { as described in plant procedures. Cracks, erosion, corrosion of embedded steel, and } \\
\text { concrete spalling have been observed. Periodic inspections of the circulating water } \\
\text { pumphouse have been an ongoing program. Divers perform inspections during } \\
\text { refueling outages. Minor degradation of these concrete structures has been found and } \\
\text { recorded. Zebra mussels are periodically removed from the forebay areas. }\end{array}$ \\
\hline $\begin{array}{l}\text { Systems } \\
\text { Monitoring } \\
\text { Program }\end{array}$ & $\begin{array}{l}\text { This AMP manages aging effects for normally } \\
\text { accessible, external surfaces of piping, tanks, and } \\
\text { other components and equipment within the scope } \\
\text { of license renewal. }\end{array}$ & $\begin{array}{l}\text { Industry: } \\
\text { Not discussed } \\
\text { Plant Specific: } \\
\text { A PBNP review of documentation for seven systems within the scope of license } \\
\text { renewal indicated that these walkdowns usually result in the initiation of corrective } \\
\text { Work Orders for the repair of minor leaks from both flanged connections and valve } \\
\text { stem packing, degraded grout under pumps, or pipe supports. }\end{array}$ \\
\hline Tank Internal & This AMP is a new plant-specific program that & Industry: \\
\hline
\end{tabular}




\begin{tabular}{|c|c|c|}
\hline $\begin{array}{c}\text { Aging } \\
\text { Management } \\
\text { Program }\end{array}$ & $\begin{array}{c}\text { Description of Aging Management Program } \\
\text { (AMP) }\end{array}$ & Operating Experience \\
\hline $\begin{array}{l}\text { Inspection } \\
\text { Program }\end{array}$ & $\begin{array}{l}\text { manages aging effects on the (a) internal surfaces } \\
\text { of carbon steel tanks, and (b) inaccessible external } \\
\text { surfaces of carbon steel tanks (i.e., tank bottoms) } \\
\text { where wall thickness measurements may be taken } \\
\text { from inside the tank to detect external degradation } \\
\text { (e.g., using ultrasonic techniques). }\end{array}$ & $\begin{array}{l}\text { A coated carbon steel Refueling Water Storage Tank (RWST) was found to have } \\
\text { coating degradation. } \\
\text { Plant Specific: } \\
\text { The south Condensate Storage Tank was internally inspected in January } 2000 \text { with } \\
\text { minor surface rust observed on the floor of the tank and corrosion through the tank } \\
\text { coating observed on the lower } 6 \text { to } 8 \text { inches of the tank wall. Inspection of the north } \\
\text { Condensate Storage Tank revealed minor surface rust similar to that found in the other } \\
\text { tank. }\end{array}$ \\
\hline $\begin{array}{l}\text { Thimble Tube } \\
\text { Inspection } \\
\text { Program }\end{array}$ & $\begin{array}{l}\text { This AMP is an existing plant-specific program that } \\
\text { manages aging effects for incore instrument } \\
\text { thimble tubes. This program requires periodic eddy } \\
\text { current testing of thimble tubes and contains criteria } \\
\text { for determining sample size, inspection frequency, } \\
\text { flaw evaluation, and corrective action, in } \\
\text { accordance with NRC Bulletin } 88-09 \text {. }\end{array}$ & $\begin{array}{l}\text { Industry: } \\
\text { NRC Bulletin } 88-09 \text { was issued in response to the thinning of thimble tubes } \\
\text { experienced at several Westinghouse pressurized water reactors. } \\
\text { Plant Specific: } \\
5 \text { tubes on Unit } 1 \text { have been replaced due to wear since } 1985 \text {. One of these } 5 \text { tubes had } \\
\text { been capped, one other showed significant wear and would require repositioning or } \\
\text { capping prior to the next inspection, while the other } 3 \text { were replaced since they } \\
\text { indicated the most wear when compared to the remaining tubes. }\end{array}$ \\
\hline $\begin{array}{l}\text { Water } \\
\text { Chemistry } \\
\text { Control } \\
\text { Program }\end{array}$ & $\begin{array}{l}\text { This AMP manages aging effects by controlling the } \\
\text { internal environment of systems and components. } \\
\text { Primary, borated and secondary water systems are } \\
\text { included in the scope of the program. The program } \\
\text { conforms to the guidelines in EPRI TR-105714 and } \\
\text { TR-102134. }\end{array}$ & $\begin{array}{l}\text { Industry: } \\
\text { Not discussed } \\
\text { Plant Specific: } \\
\text { Review of plant-specific operating experience indicates that the chemistry program is } \\
\text { performing its function of mitigating aging effects. No reports were found that } \\
\text { attributed water chemistry as the cause of component deterioration, showing signs of } \\
\text { aging effects, or failing to perform its function. }\end{array}$ \\
\hline
\end{tabular}





\section{CONCLUSIONS}

This report describes the research effort performed for the Year 1 scope of work under a collaboration effort between BNL and KAERI. This research focused on collecting and reviewing degradation occurrences in US NPPs and identifying important aging characteristics needed for the seismic capability evaluations that will be performed in the subsequent evaluations in the years that follow. The report presents the results of statistical and trending analyses of this data and compares the results to prior aging studies. In addition, this report provides a description of current regulatory requirements, regulatory guidance documents, generic communications, industry standards and guidance, and past research related to aging degradation of SSCs. This section of the report presents the conclusions reached from this research effort, which includes a summary of the findings from the identification and evaluation effort of degradation occurrences, an assessment of the degradation trending results, and insights into the important aging characteristics that should be considered in the tasks to be performed in the Year 2 through 5 research efforts.

A survey of degradation occurrences for structures and passive components (SPCs) was conducted using recent licensee event reports (LERs) and recent generic letters, bulletins, information notices, and license renewal applications. The study also included trending analysis of the degradation occurrence records (DORs) obtained from LERs, in combination with data reported in NUREG/CR-6679. The goal of this study was to identify any new degradation trends and to determine whether the findings reported in NUREG/CR-6679 still hold. The ultimate goal of this study is to assist in identifying those degraded SPCs that are significant to plant safety for use in the development of seismic capability evaluation technology for degraded structures and components.

Past studies on aging-related degradation were critically evaluated; part of the evaluation presented in this report included a summary of the relevant information presented in NUREG/CR-6679. A description of regulatory requirements, NRC regulatory guidance, NRC programs, industry programs, and international research efforts were presented. Also included in this report is a brief discussion of the NRC sponsored multi-year research program on agingrelated degradation, which was performed by BNL recently. This information provides an extensive literature overview that is beneficial to the current BNL-KAERI collaborative effort.

After a thorough examination of possible approaches for performing the review of 4,323 LERs from 1999 to April 16, 2008, software tools were developed to assist the review process of the LERs. These tools assisted in downloading all LERs automatically and in speeding up the review process significantly. The completion of this study benefited greatly from the development of these tools.

Ten component categories were identified for this study which consist of anchorage, concrete, containment, exchanger, filter, piping system, reactor pressure vessel (RPV), structural steel, tank, and vessel. A total of 223 DORs were identified for the ten component categories, and the relevant information is summarized in the primary information table and the secondary information table presented in Section 3 of this report. The results of this study demonstrated that piping systems have the most degradation occurrences reported in LERs, about $36 \%$ of the total DORs. Exchangers and RPVs have the next two largest numbers of degradation occurrences, representing about $22 \%$ and $17 \%$ of the total DORs, respectively. The other seven component categories represent less than $25 \%$ of the total DORs. It should be noted that part of the explanation for these results is related to the number of components that are found at a NPP. For example, there are many piping systems and many of these systems are quite long, as compared 
to a more limited number of other components such as containments and tanks. Also, the environment for certain components such as RPVs is much harsher, and therefore, leads to a greater number of degradation occurrences. It was also found that LERs do not report a significant number of structural type components, such as containments, structural steel, concrete, and anchorages. The number of structural DORs reported in LERs was judged to be lower than the actual structural degradations. However, this does not indicate in any way that structural degradations are of less significance to plant safety. The lower number of structural degradations may be partially attributed to the fact that they may be difficult to identify and the degradations may not have been judged to reach a level that would require a formal submittal of an LER to the NRC.

Taking into consideration that the number of DORs for the piping system was limited during the LER review in NUREG/CR-6679, the current study concluded that exchangers, piping system, and RPVs were found to be the first three categories with the greatest number of DORs. The distribution of DORs over time was somewhat cyclic, which is judged to be partially influenced by inspection intervals that often are scheduled at refueling or are required by special NRC mandatory inspection requirements. Distribution of the average DORs by plant age at event (PAAE) showed that the average number of DORs per plant increase as the plant gets older, with a slightly higher rate for older plants as shown by the steeper slope using the LER 1999-2007 results. The slightly higher degradation rate using more recent LERs reflects the fact that older plants show in general more degradation occurrences. Omitting other factors that may affect the accuracy of the trending analysis, the older plants have about 3 times higher average DORs/year than the younger plants, although the absolute value of the average DORs is considered to be very small (1 1.8 occurrences/plant/year for those plants which have degradation occurrences).

Using the NUREG/CR-6679 (1985 to 1997) results for all sources of degradation occurrences, the NUREG/CR-6679 results based only on LER data, and the current LER study (1999-2008) results, cracking was found to be the most predominant aging effect. Stress corrosion cracking (SCC) was the most significant aging mechanism for all three data series. Primary water stress corrosion cracking (PWSCC) was found to be the major contributor to SCC based on the LER results and intergranular SCC (IGSCC) was the major contributor if the entire NUREG/CR-6679 data was used. The LER 1999-2008 data series also showed that fatigue was the second significant aging mechanism, indicating the possibility that some components might be approaching their fatigue life as NPPs got older. Moisture, organisms, chemical attack, and foreign object were shown to be less important mechanisms using the LER 1998-2008 series. The system that was most vulnerable to degradation is the reactor coolant systems (RCS), as expected because the RCS includes many subcomponents that are constantly subjected to harsh environments such as high temperature, high pressure, high fluid velocity, boron acid, radiation, etc. Also, the RCS receives close scrutiny in terms of inspections and examination in view of the safety importance of the system.

Review of recent generic communications (generic letters, bulletins, and information notices) indicated that exchangers, RPVs, and piping systems are the top three components that generic correspondences address. Generic communications discuss more structural type components than the LERs do. Documents related to LRAs are also useful because they identify applicable aging effects, discuss industry and plant specific operating experience, and describe instances of aging degradation of structures and components at their plants. Two recent representative LRAs, Palisades and Point Beach Units $1 \& 2$, were chosen for review. Regarding aging-degradations, the information presented in LRAs is not as detailed as in the LERs. These reviews did not yield adequate data for a direct comparison to those found using LERs. However, these reviews confirmed qualitatively the findings using LERs, because in many cases the plant-specific 
information in the LRAs, for the more significant degradation occurrences, was already in the LERs for that plant.

Utilizing the trending analysis from previous DORs reported in NUREG/CR-6679, DORs from the review of LERs described in this report, and from the general observations obtained from the review of recent representative generic letters, bulletins, information notices, and license renewal applications, it is concluded that the patterns of degradation occurrences have not significantly changed from past studies, although signs of a slight increase in the number of DORs have been observed. Although the LERs do not report many structural degradations based on the reporting requirements governed by 10 CFR 50.73, structural degradations are expected to be a factor as plants age, and are important to plant safety when extreme environmental demands such as large earthquakes are considered. It can be further stated that the conclusions reached from the NUREG/CR-6679 study, regarding the list of components whose age-related degradation is significant to plant safety and the characteristics of aging degradation, are still valid for the collaborative research being performed by BNL and KAERI. 



\section{REFERENCES}

10 CFR 50.55a Codes and Standards.

10 CFR 50.65, Requirements for Monitoring the Effectiveness of Maintenance at Nuclear Power Plants.

10 CFR 50.72, Immediate Notification Requirements for Operating Nuclear Power Reactors.

10 CFR 50.73, Licensee Event Report System.

10 CFR Part 50, Domestic Licensing of Production and Utilization Facilities, Appendix J, Primary Reactor Containment Leakage Testing for Water-Cooled Power Reactors.

10 CFR Part 51, Environmental Protection Regulations for Domestic Licensing and Related Regulatory Functions.

10 CFR Part 54, Requirements for Renewal of Operating Licenses for Nuclear Power Plants.

ACI 201.1R-68, "Guide for Making a Condition Survey of Concrete in Service," American Concrete Institute, Detroit, Michigan, Revised 1984.

ACI 201.2R-77, "Guide to Durable Concrete," American Concrete Institute, Detroit, Michigan, Reapproved 1982.

ACI 207.3R-79, "Practices for Evaluation of Concrete in Existing Massive Structures for Service Conditions," American Concrete Institute, Detroit, Michigan, Revised 1985.

ACI 222R-89, "Corrosion of Metals in Concrete," American Concrete Institute, Detroit, Michigan, 1989.

ACI 224.1R-93, "Causes, Evaluation, and Repair of Cracks in Concrete Structures," American Concrete Institute, Detroit, Michigan, 1993.

ACI 224R-90, "Control of Cracking in Concrete Structures," American Concrete Institute, Detroit, Michigan, 1990.

ACI 349.3R-96, “ Evaluation of Existing Nuclear Safety-Related Concrete Structures, American Concrete Institute, Detroit, Michigan, March 1996.

ASCE 11-90, Guideline for Condition Assessment of Existing Buildings, American Society of Civil Engineers, New York, NY, Copyright 1991.

American Society of Mechanical Engineers, Boiler and Pressure Vessel Code, 1992 edition, Section XI, Rules for Inservice Inspection of Nuclear Power Plant Components, with 1992 addenda, New York.

NUREG/CR-6876, Risk-informed assessment of degraded buried piping systems in nuclear power plants, Braverman, J.I., DeGrassi G., Martinez-Guridi, G., Morante, R.J., Hofmayer, C.H., Brookhaven National Laboratory, June 2005. 
NUREG/CR-6679, Assessmesnt of age-related degradation of structures and passive components for U.S. nuclear power plant, Braverman, J.I., Hofmayer, C.H., Morante, R.J., Shteyngart, S., and Bezler, P., Brookhaven National Laboratory, July 2000.

NUREG/CR-6715, Probability-based evaluation of degraded reinforced concrete components in nuclear power plants, Braverman, J.I., Miller, C.A., Ellingwood, B.R., Naus, D.J., Hofmayer, C.H., Shteyngart, S., and Bezler, P., Brookhaven National Laboratory, April 2001.

IE Bulletin 80-11, “Masonry Wall Design,” U.S. Nuclear Regulatory Commission, May 8, 1980.

Information Notice No. 87-67, "Lesson Learned from Regional Inspections of Licensee Actions in Response to IE Bulletin 80-11," U.S. Nuclear Regulatory Commission, December 31, 1987.

NEI 95-10, Revision 6, "Industry Guideline for Implementing the Requirements of 10 CFR Part 54 - The License Renewal Rule,” Nuclear Energy Institute, June 2005.

NRC Inspection Manual - Inspection Procedure 62002, "Inspection of Structures, Passive Components, and Civil Engineering Features at Nuclear Power Plants," December 31, 1996.

NRC Inspection Manual - Inspection Procedure 62003, "Inspection of Steel and Concrete Containment Structures at Nuclear Power Plants," June 11, 1997.

NRC Inspection Manual - Inspection Procedure 62706, “Maintenance Rule,” August 31, 1995.

NUMARC 93-01, Revision 2, "Industry Guideline for Monitoring the Effectiveness of Maintenance at Nuclear Power Plants," April 1996.

NUREG/CR-6424, "Report on Aging of Nuclear Power Plant Reinforced Concrete Structures," by D. J. Naus and C. B. Oland, Oak Ridge National Laboratory, March 1996.

NUREG/CR-6490, "Nuclear Power Plant Generic Aging Lessons Learned (GALL)," by K. E. Kasza et al, Argonne National Laboratory, December 1996.

NUREG-1022, "Event reporting guidelines 10 CFR 50.72 and 50.73," Rev. 2, U.S. Nuclear Regulatory Commission, Washington, DC, 20555, October 2000.

NUREG-1144, Revision 2, "Nuclear Plant Aging Research (NPAR) Program Plan," U.S. Nuclear Regulatory Commission, Washington, D.C., June 1991.

NUREG-1350, “2007-2008 information digest,” Vol. 19, U.S. Nuclear Regulatory Commission, Washington, DC, 20555, August 2007.

NUREG-1377, Revision 4, "NRC Research Program on Plant Aging: Listing and Summaries of Reports Issued Through September 1993,” by J. P. Vora, December 1993.

NUREG-1522, “Assessment of Inservice Condition of Safety-Related Nuclear Plant Structures," by H. Ashar and G. Bagchi, U.S. Nuclear Regulatory Commission, Washington, D.C., June 1995.

NUREG-1800 (SRP-LR), Revision 1, "Standard Review Plan for the Review of License Renewal Applications for Nuclear Power Plants," U.S. Nuclear Regulatory Commission, Washington, D.C., September 2005. 
NUREG-1801, Revision 1, Generic Aging Lessons Learned (GALL) Report, U.S. Nuclear Regulatory Commission, Washington, D.C., September 2005.

Park, Y., Draft Report - "Effects of Aging Degradation on Seismic Performance of Reinforced Concrete Structures: Summary of Japanese Literature in Related Areas," Brookhaven National Laboratory, September 1998.

Regulatory Guide 1.127, Revision 1, "Inspection of Water-Control Structures Associated with Nuclear Power Plants," U.S. Nuclear Regulatory Commission, Washington, D.C., March 1978.

Regulatory Guide 1.160, Revision 2, "Monitoring the Effectiveness of Maintenance at Nuclear Power Plants,” U.S. Nuclear Regulatory Commission, Washington, D.C., March 1997.

Regulatory Guide 1.188, Revision 1, "Standard Format and Content for Applications to Renew Nuclear Power Plant Operating Licenses," U.S. Nuclear Regulatory Commission, Washington, D.C., September 2005.

Regulatory Guide 1.35, Revision 3, "Inservice Inspection of Ungrouted Tendons in Prestressed Concrete Containments," U.S. Nuclear Regulatory Commission, Washington, D.C., July 1990.

Regulatory Guide 1.35.1, "Determining Prestressing Forces for Inspection of Prestressed Concrete Containments," U.S. Nuclear Regulatory Commission, Washington, D.C., July 1990. 